\title{
OPTIMIZING EXPOSURE OF ORAL \\ TARGETED THERAPIES IN ONCOLOGY
}

\author{
TOWARDS PRECISION DOSING
}

Steffie Groenland 
The research described in this thesis was performed at the Department of Clinical Pharmacology, Division of Medical Oncology of the Netherlands Cancer Institute - Antoni van Leeuwenhoek, Amsterdam, the Netherlands, in collaboration with other institutes.

Printing of this thesis was financially supported by the Netherlands Cancer Institute.

ISBN: 978-94-6416-333-9

Design \& lay-out: Marco Kortekaas \& Steffie Groenland

Printing: Ridderprint

(c) Stefanie L. Groenland, 2020 


\title{
Optimizing exposure of oral targeted therapies in oncology \\ towards precision dosing
}

\author{
Optimaliseren van de blootstelling aan orale doelgerichte \\ therapieën binnen de oncologie \\ op weg naar doseren op maat \\ (met een samenvatting in het Nederlands)
}

\section{Proefschrift}

ter verkrijging van de graad van doctor aan de Universiteit Utrecht op gezag van de rector magnificus, prof. dr. H.R.B.M. Kummeling, ingevolge het besluit van het college voor promoties in het openbaar te verdedigen op donderdag 4 februari 2021 des middags te 2.30 uur

door

Stefanie Lianne Groenland geboren op 20 oktober 1991 te Obdam 
Promotoren:

Prof. dr. A.D.R. Huitema

Prof. dr. J.H. Beijnen

Copromotor:

Dr. N. Steeghs 
La médecine c'est guérir parfois, soulager souvent, consoler toujours Ambroise Paré 


\section{TABLE OF CONTENTS}

Preface and thesis outline $\quad 9$

PART I - Rationale for precision dosing 17

Chapter 1 - Individualized dosing of oral targeted therapies in oncology 19 is crucial in the era of precision medicine

Eur J Clin Pharmacol 2019; 75: 1309-1318

Chapter 2 - Demonstrating clinical relevance of individualized dosing of oral targeted therapies in oncology - the path to precision dosing

Submitted

PART II - Exposure-response analyses

Chapter 3 - Exposure-response analyses of abiraterone and its

metabolites in real-world patients with metastatic castration-resistant

prostate cancer

Prostate Cancer Prostatic Dis 2020; 23: 244-251

Chapter 4 - Exposure-response analyses of anaplastic lymphoma

kinase inhibitors crizotinib and alectinib in non-small cell lung cancer patients

Clin Pharmacol Ther. Online ahead of print 19 Jul 2020

Chapter 5 - Exposure-response analyses of dabrafenib and trametinib

in melanoma patients

Manuscript in preparation

PART III - Precision dosing in clinical practice

Chapter 6 - Therapeutic drug monitoring of oral anticancer drugs: the

Dutch Pharmacology Oncology Group - Therapeutic Drug Monitoring protocol for a prospective study

Ther Drug Monit 2019; 41: 561-567

Chapter 7 - Therapeutic drug monitoring based precision dosing of oral

targeted therapies in oncology: a prospective multicenter study

Interim analysis

Chapter 8 - Therapeutic drug monitoring of imatinib in patients with gastro-intestinal stromal tumours - results from daily clinical practice Eur J Cancer 2020; 136: 140-148

Chapter 9 - Harnessing soft tissue sarcoma with low-dose pazopanib a matter of blood levels

BMC Cancer 2018; 18: 1200 
Chapter 10 - Therapeutic drug monitoring of oral anti-hormonal drugs

in oncology

Clin Pharmacokinet 2019; 58: 299-308

\section{PART IV - Alternative strategies for precision dosing}

Chapter 11 - Concomitant intake of abiraterone acetate and food to increase pharmacokinetic exposure: real life data from a therapeutic drug monitoring programme Eur J Cancer 2020; 130: 32-39

Chapter 12 - Cost-neutral optimization of pazopanib exposure by splitting intake moments: a prospective pharmacokinetic study in cancer patients

Clin Pharmacokinet 2020; 59: 941-948

Chapter 13 - Individualized dosing of pazopanib - using cost-neutral 239 interventions to optimize exposure Interim analysis

Chapter 14 - Clinical pharmacokinetics and pharmacodynamics of the cyclin dependent kinases 4 and 6 inhibitors palbociclib, ribociclib and abemaciclib Clin Pharmacokinet. Online ahead of print 8 Oct 2020

Chapter 15 - Effect of the moderate CYP3A4 inhibitor erythromycin on the pharmacokinetics of palbociclib Interim analysis

Chapter 16 - Determination of the absolute bioavailability of oral imatinib using a stable isotopically labeled intravenous imatinib-d8 microdose

Eur J Clin Pharmacol 2020; 76: 1075-1082

Conclusions and perspectives

Nederlandse samenvatting

\section{Appendices}

Author affiliations

List of publications

Dankwoord

Curriculum vitae 

Preface 



\section{PREFACE}

A major breakthrough in the treatment of cancer has been the development of imatinib, which has widely been praised as the magic anticancer bullet. ${ }^{1}$ In the era of targeted therapies that we have come to know ever since, the number of oral targeted therapies that is approved each year increases exponentially. With the advent of these small molecules, that inhibit specific driver proteins in growth signal transduction pathways, the focus has increasingly shifted towards precision medicine, by selecting the right drug for individual patients based on molecular characteristics of their tumor. However, nowadays these drugs are still administered using a one-size-fits-all fixed dosing approach, while convincing arguments advocate for precision dosing instead.

First, there is a weak basis for the registered dose, as the recommended dose is determined in phase I studies, that typically include only a small number of patients and mainly focus on toxicity instead of efficacy. ${ }^{2}$ Second, compared with intravenously administered classical cytotoxics, this new class of orally administered drugs shows a higher variability in pharmacokinetic (PK) exposure, due to differences in drug absorption. Additionally, many oral targeted therapies are characterized by complex pharmacological profiles, due to their poor formulation and complex metabolism, which adds to the high interindividual variability in exposure as well. ${ }^{3}$ Consequently, fixed dosing results in a wide range of drug concentrations between patients. ${ }^{4}$ Third, and most importantly, many of these new oral targeted therapies have a narrow therapeutic window. ${ }^{5}$

As a result, the problem with the currently used fixed dosing regimen is that $\pm 30 \%$ of patients are being underdosed and thus at risk of suboptimal treatment efficacy, whereas another $\pm 15 \%$ of patients are currently being overdosed, potentially resulting in unnecessary treatment-related toxicities. ${ }^{5,6}$ Hence, rational precision medicine would not only include selecting the right drug, but also selecting the right dose. Precision dosing can be achieved by selecting the right starting dose for each individual (group of) patient(s), i.e. "right-dose-first-time" paradigm, after which this dose can be further optimized by PK-guided dosing, i.e. adjusting the dose based on measured drug concentrations.

\section{THESIS OUTLINE}

This thesis focuses on the rationale (Part I), the evidence (Part II) and the clinical application (Part III and IV) of precision dosing of oral targeted therapies in oncology.

Part I outlines the rationale for precision dosing of oral anticancer drugs. In Chapter 1, seven prerequisites for the rational application of PK-guided dosing are discussed for oral targeted therapies. In Chapter 2, it is discussed how the clinical relevance of precision 
dosing in oncology can be demonstrated, and the need for randomized confirmatory trials is questioned.

Part II reports on exposure-response analyses in real-life patient cohorts. Chapter $\mathbf{3}$ for abiraterone and its active metabolites in patients with metastatic castration-resistant prostate cancer, Chapter $\mathbf{4}$ for first- and second-generation anaplastic lymphoma kinase inhibitors crizotinib and alectinib in patients with non-small-cell lung cancer, and Chapter 5 for the combination of BRAF- and MEK-inhibitor dabrafenib plus trametinib in patients with melanoma.

Part III focuses on the application of precision dosing in clinical practice. First of all, the study protocol of the Dutch Pharmacology Oncology Group - Therapeutic Drug Monitoring (DPOG-TDM) study is described in Chapter 6. The first results of this prospective nationwide study on PK-guided dosing in 600 patients with 24 different oral targeted therapies are presented in Chapter 7. In Chapter 8, the results of PK-guided dose increases of imatinib in patients with gastrointestinal stromal tumors are described, for whom PK-guided dosing is performed as part of daily clinical practice at our institute for several years now. In Chapter 9 it is illustrated how PK-guided dosing could support treatment decisions in case of toxicity by reporting on two patients who received an extremely low dose of pazopanib. Chapter $\mathbf{1 0}$ provides practical recommendations for pharmacokinetic targets to guide dosing of oral anti-hormonal drugs.

Part IV of this thesis highlights alternative strategies for precision dosing. Chapter 11 provides more detailed data on the first patients in the abiraterone cohort of the DPOGTDM study, by describing how food interventions can be used to optimize abiraterone exposure in patients with trough concentrations below the target. In Chapter 12, a pharmacokinetic crossover study investigating the effect of splitting intake moments of pazopanib from $800 \mathrm{mg}$ once daily into $400 \mathrm{mg}$ twice daily is described. Chapter 13 reports on the pazopanib cohort of the DPOG-TDM study in more detail, focusing on costneutral PK-guided interventions to optimize exposure. Chapter $\mathbf{1 4}$ focuses on cyclindependent kinase inhibitors and provides an overview of the clinical pharmacokinetics and pharmacodynamics of these drugs, including exposure-response relationships and their potential for drug-drug interactions via cytochrome P450 3A4 (CYP3A4). In Chapter 15, the effect of the moderate CYP3A4 inhibitor erythromycin on the pharmacokinetic exposure of palbociclib is investigated in a randomized crossover study, aiming to provide guidelines for clinicians on dose modifications. An important factor in the high inter- and intra-individual variability of oral targeted therapies is the bioavailability. ${ }^{3}$ In Chapter 16, a clinical study is presented in which the absolute bioavailability of imatinib is determined using an innovative design. 
Finally, the main conclusions of the presented work are discussed and placed in a broader perspective, followed by a detailed summary of the most important findings described in each chapter.

In summary, this thesis describes the rationale and opportunities for precision dosing of oral targeted therapies in oncology, with the aim of optimizing exposure for individual patients. 


\section{REFERENCES}

1. Longo DL. Imatinib changed everything. N Eng/J Med 2017; 376: 982-983.

2. Mathijssen RHJ, Sparreboom A, Verweij J. Determining the optimal dose in the development of anticancer agents. Nat Rev Clin Oncol 2014; 11: 272-281.

3. Herbrink $\mathrm{M}$, Nuijen $\mathrm{B}$, Schellens JHM, Beijnen JH. Variability in bioavailability of small molecular tyrosine kinase inhibitors. Cancer Treat Rev 2015; 41: 412-422.

4. Yu H, Steeghs N, Nijenhuis CM, Schellens JHM, Beijnen JH, Huitema ADR. Practical guidelines for therapeutic drug monitoring of anticancer tyrosine kinase inhibitors: focus on the pharmacokinetic targets. Clin Pharmacokinet 2014; 53: 305-325.

5. Verheijen RB, $\mathrm{Yu} H$, Schellens JHM, Beijnen JH, Steeghs $N$, Huitema ADR. Practical recommendations for therapeutic drug monitoring of kinase inhibitors in oncology. Clin Pharmacol Ther 2017; 102: 765-776.

6. Lankheet NAG, Desar IME, Mulder SF, Burger DM, Kweekel DM, van Herpen CML et al. Optimizing the dose in cancer patients treated with imatinib, sunitinib and pazopanib. $\mathrm{Br} J$ Clin Pharmacol 2017; 83: 2195-2204. 




\section{PART I}

Rationale for precision dosing 


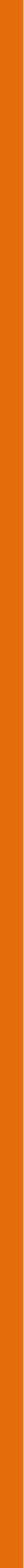




\section{ABSTRACT}

\section{Purpose}

While in the era of precision medicine, the right drug for each patient is selected based on molecular tumor characteristics, most novel oral targeted anticancer agents are still being administered using a one-size-fits-all fixed dosing approach. In this review, we discuss the scientific evidence for dose individualization of oral targeted therapies in oncology, based on therapeutic drug monitoring (TDM).

\section{Methods}

Based on literature search and our own experiences, seven criteria for drugs to be suitable candidates for TDM will be addressed: 1) absence of an easily measurable biomarker for drug effect; 2) long-term therapy; 3) availability of a validated sensitive bioanalytical method; 4) significant variability in pharmacokinetic exposure; 5) narrow therapeutic range; 6 ) defined and consistent exposure-response relationships; 7) feasible dose-adaptation strategies.

\section{Results}

All of these requirements are met for most oral targeted therapies in oncology. Also, prospective studies have already shown TDM to be feasible for imatinib, pazopanib, sunitinib, everolimus and endoxifen.

\section{Conclusions}

In order to realize the full potential of personalized medicine in oncology, patients should not only be treated with the right drug, but also at the right dose. TDM could be a suitable tool to achieve this. 


\section{INTRODUCTION}

Many new oral targeted therapies have become available in oncology over the past two decades. As a result, the treatment paradigm has partly shifted from a one-size-fits-all approach into precision medicine, in which the right drug is selected based on molecular characteristics of the tumor.

Dose finding of these new oral targeted therapies, however, has simply been copied from classical intravenous cytotoxic drugs. In traditional phase I dose escalation studies, which generally enroll only few patients (median sample size of 26 patients ${ }^{1}$ ), doses are increased until dose-limiting toxicities occur. This maximum tolerated dose (MTD), at which typically only 3-6 patients have been treated, is then used in all further studies, leading to a one-size-fits-all fixed dosing strategy. ${ }^{2}$ However, pharmacokinetic characteristics of these new oral targeted therapies suggest individualized dosing would be far more rational.

Although one might think drug selection based on molecular diagnoses makes any further dose individualization superfluous, it seems logical to combine these two approaches to realize the full potential of personalized medicine (Figure 1). Currently, all patients are treated at a standard fixed dose, resulting in low pharmacokinetic exposure and thus suboptimal treatment in a substantial proportion of patients. This subtherapeutic treatment is senseless, especially with these expensive drugs. Therefore, we believe that the current fixed dosing paradigm should be left. Therapeutic drug monitoring (TDM), which is individualized dosing based on measured plasma concentrations of the drug, can be used to select the right dose for each individual patient. In case of pharmacokinetic exposure below the predefined efficacy threshold and acceptable toxicities, pharmacokinetically guided interventions will be performed. These could include absolute dose increments or alternative interventions to increase pharmacokinetic exposure (i.e. concomitant intake with food in case of a clinically relevant food effect ${ }^{3}$ or splitting intake moments in case of saturable absorption ${ }^{4}$ ). Although TDM is widely applied in clinical practice for many drug classes, such as antibiotics, anticonvulsants, and immunosuppressants, it is still being very limitedly applied in oncology.

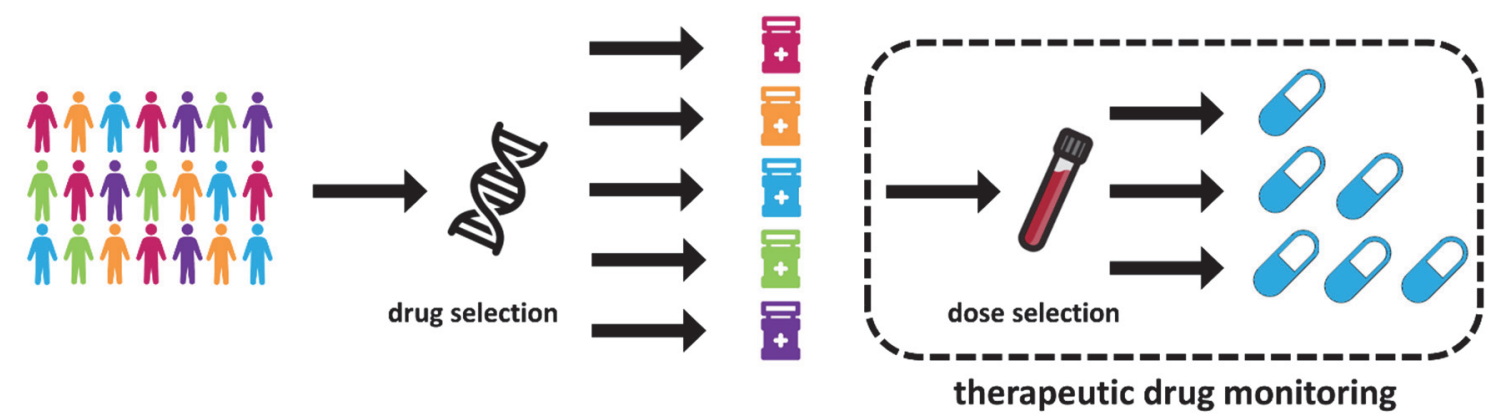

Figure 1 - Schematic overview of how precision medicine can be combined with dose individualization by therapeutic drug monitoring 
Previous reviews have summarized the literature for TDM of individual oral anticancer drugs. ${ }^{5-9}$ In this review, we will address the scientific evidence for dose individualization by TDM of oral targeted therapies in general. Table 1 provides an overview of approved oral targeted therapies in oncology.

Table 1 - Overview of oral targeted therapies in oncology (2019)

\begin{tabular}{ll}
\hline Group & Drugs \\
\hline ALK inhibitors & alectinib, brigatinib, ceritinib, crizotinib, lorlatinib \\
\hline Anti-hormonal drugs & $\begin{array}{l}\text { abiraterone, anastrozole, apalutamide, enzalutamide, } \\
\text { exemestane, letrozole, tamoxifen }\end{array}$ \\
\hline Bcr-Abl inhibitors & bosutinib, dasatinib, imatinib, nilotinib, ponatinib \\
\hline BRAF inhibitors & dabrafenib, encorafenib, vemurafenib \\
\hline BTK inhibitors & ibrutinib \\
\hline CDK 4/6 inhibitors & abemaciclib, palbociclib, ribociclib \\
\hline EGFR inhibitors & dacomitinib, erlotinib, gefitinib, osimertinib \\
\hline EGFR / Her2 inhibitors & afatinib, neratinib, lapatinib \\
\hline FLT3 inhibitors & gilteritinib, midostaurin \\
\hline HDAC inhibitors & panobinostat, vorinostat \\
\hline JAK inhibitors & ruxolitinib \\
\hline MEK inhibitors & binimetinib, cobimetinib, trametinib \\
\hline mTOR inhibitors & everolimus \\
\hline NTRK inhibitors & larotrectinib \\
\hline PARP inhibitors & olaparib, niraparib, rucaparib, talazoparib \\
\hline PI3K inhibitors & copanlisib, duvelisib, idelalisib \\
\hline VEGFR inhibitors & axitinib, cabozantinib, lenvatinib, nintedanib, pazopanib, \\
\hline regorafenib, sorafenib, sunitinib, tivozanib, vandetanib
\end{tabular}

$A L K=$ anaplastic lymphoma kinase; $B C r-A b l=$ breakpoint cluster region-Abelson fusion protein; $B R A F=$ serine/threonine-protein kinase B-Raf; BTK = Bruton's tyrosine kinase; $C D K=$ cyclin dependent kinase; $E G F R=$ epidermal growth factor receptor; Her2 = human epidermal growth factor receptor 2; FLT3 = FMS-like tyrosine kinase 3; HDAC = histone deacetylase; JAK = Janus-associated kinase; $M E K=$ mitogenactivated protein kinase; $m T O R=$ mammalian target of rapamycin; NTRK = neurotrophic tyrosine kinase; $P A R P=$ poly ADP ribose polymerase; $P I 3 K=$ phosphoinositide 3 kinase; $V E G F R=$ vascular endothelial growth factor receptor

\section{CRITERIA FOR RATIONAL USE OF THERAPEUTIC DRUG MONITORING}

For drugs to be suitable candidates for TDM, the following requirements have previously been proposed $^{10-14}$ :

1. absence of an easily measurable biomarker for drug effect;

2. long-term therapy;

3. availability of a validated sensitive bioanalytical method;

4. significant variability in pharmacokinetic exposure;

5. narrow therapeutic range; 
6. defined and consistent exposure-response relationships;

7. feasible dose-adaptation strategies.

In the following paragraphs, each of these requirements will be discussed and it will be assessed whether they are met in the case of oral targeted therapies in oncology.

\section{Absence of an easily measurable biomarker for drug effect}

If more convenient, accurate, and precocious biomarkers for drug response would be available, these would make TDM superfluous. However, while toxicity can easily be measured, for efficacy, these biomarkers are generally not available (yet) and response evaluations with regard to antitumor efficacy are often based on radiological assessments, which are not performed timely enough to be a good biomarker. Imaging is usually performed every 8 to 12 weeks, while ideally dose adjustments should be made at an early stage (i.e. within 14 days). Also, once tumor progression is observed on radiological scans, resistant clones of tumor cells have already emerged and dose adjustments will probably be too late at this moment. Although for some tumor types blood-based tumor markers exist (e.g. cancer antigen 125 in ovarian cancer or carcinoembryonic antigen in colorectal cancer), these are not accurate enough to predict treatment response and to base treatment decisions upon. ${ }^{15}$ The same holds true for other potential biomarkers available for oral targeted therapies including diastolic blood pressure for axitinib and skin rash for epidermal growth factor receptor inhibitors such as erlotinib and gefitinib. ${ }^{16-18}$ Complete cytogenetic response in case of hematologic malignancies and prostate-specific antigen (PSA) in the case of prostate cancer are the only examples of biomarkers that can accurately predict response to treatment and that are used in clinical practice for this purpose. ${ }^{19,20}$ Apart from these exceptions, the first requirement for TDM is met for most combinations of targeted therapies and tumor types.

\section{Long-term therapy}

Treatment should be long enough to allow sufficient time for dose adjustments to be made. As the mean treatment duration of targeted therapies is several months, while only few days to weeks are needed to reach steady-state concentrations, there is sufficient time to perform TDM. The time to steady-state concentrations depends on the elimination half-life $\left(\mathrm{t}_{1 / 2}\right)$ of a drug, which is typically around 20-30 hours for most oral anticancer drugs, although for some compounds this is markedly longer (e.g. enzalutamide $( \pm 6$ days $^{21}$ ) and endoxifen, which is the active metabolite of tamoxifen $\left( \pm 2\right.$ weeks $\left.{ }^{22}\right)$ ). After four to five times the $t_{1 / 2}$, steady-state concentrations have been attained.

\section{Availability of a validated sensitive bioanalytical method}

In order to perform dose individualization based on pharmacokinetic exposure, bioanalytical assays to measure plasma concentrations should be available. Liquid chromatography-tandem mass spectrometry (LC-MS/MS) is usually applied for 
quantification of these drugs and validated assays are available for almost all oral anticancer drugs at a reasonable price. Since LC-MS/MS is a labor-intensive method and many different targeted therapies will be used in routine clinical practice, combining multiple drugs into one bioanalytical assay might be useful. ${ }^{23-25}$ To implement TDM into routine clinical practice, an adequate infrastructure for sample collection and shipment should be in place, with a short turn-over and reporting time. In addition, dried blood spot (DBS) sampling could offer a more patient friendly sampling approach, as patients can obtain their blood samples themselves at home instead of having to visit the hospital. Whole blood samples can be collected by a finger prick at a paper DBS card, which can then be send to the laboratory by regular mail. DBS assays are already available for several oral anticancer drugs. ${ }^{26-33}$ Also, commercial automated immunoassays could facilitate measurement in routine clinical practice, for example of imatinib. ${ }^{34}$

\section{Significant variability in pharmacokinetic exposure}

The fourth requirement for TDM comprises a marked variability in pharmacokinetic exposure. Otherwise, when pharmacokinetic exposure would be predictable and similar for all patients, there would be no need for dose individualization.

Oral targeted therapies typically exhibit a large interindividual variability in pharmacokinetic exposure in the range of $24-84 \%$, providing a strong rationale for TDM. ${ }^{35-}$ ${ }^{40}$ Reasons for this high interindividual variability include differences in absorption, which could be influenced by the poor bioavailability of these drugs, potential food effects, or the use of drugs that alter the stomach $\mathrm{pH}$ (i.e. proton pump inhibitors and $\mathrm{H}_{2}$ receptor antagonists); interactions with concomitant medication (e.g. via cytochrome P450 enzymes such as CYP3A4); pharmacogenetics (i.e. patients harboring polymorphisms of cytochrome P450 enzymes or ABC-transporters); hepatic and renal function; body composition and patient adherence. ${ }^{2,41}$

While data on interindividual variability are widely available, reports on intra-individual variability are sparse. ${ }^{42}$ Poor formulations of most oral targeted therapies result in a low bioavailability and thus a high inter- and intra-individual variability. ${ }^{43,44}$ The intra-individual variability should be judged taking into account the interindividual variability as well. For example, abiraterone has an intra-individual variability of $33 \%$, while the interindividual variability is higher (i.e. $46 \%$ ). ${ }^{37}$ The same holds true for vemurafenib, which has an intraand interindividual variability of $28 \%$ and $41 \%$, respectively..$^{38}$ Unfortunately, these data are not available for all oral targeted therapies. Therefore, Chatelut et al. advocate intraindividual variability should ultimately be characterized before registration of new drugs. ${ }^{42}$ Although assessment of the intra-individual variability for registration purposes might be challenging, especially in the context of oncological patients with potentially fluctuating (patho-) physiological states, we do propose that efforts should be made to quantify the intra-individual variability. 
It is important to take the source of variability into account when deciding on the interval of sampling. When the major source of variability is interindividual variability, a single measurement or rare measurements would be sufficient. When the main source of variability is intra-individual variability, it depends on the origin. If the origin of intraindividual variability is random from dose to dose (e.g. due to poor formulation), TDM might not be useful, as a single sample would then be of limited value. If the intraindividual variability is caused by an identifiable reason (e.g. concomitant medication or fluctuations in (patho-)physiological conditions), more frequent sampling might be needed. In this case, the sampling interval should be oriented at the change of the condition (e.g. new concomitant medication). Regardless of the source of variability, it is important to continue sampling throughout therapy, since many factors that can influence pharmacokinetic exposure may vary over time (e.g. drug-drug interactions and compliance).

To conclude, variability in pharmacokinetic exposure of oral targeted therapies is definitely sufficiently high to meet the requisite.

\section{Narrow therapeutic range}

When the window between therapeutic and toxic concentrations is small, dose titration is important to minimize the risk of either ineffective treatment or unnecessary toxicities. The fact that $>50 \%$ of the oral targeted therapies have a recommended dose equal to the maximum tolerated dose (MTD), indicates these drugs have a narrow therapeutic index. ${ }^{45}$ An exception to this is drugs with a plateau in the exposure-response curve that are dosed at the flat end of this curve, as might be the case for cabozantinib and pazopanib. ${ }^{46,47}$ At the currently used fixed doses, $\pm 30 \%$ of patients are being under dosed (e.g. for abiraterone, imatinib, pazopanib, sunitinib, and vemurafenib), associated with decreased efficacy, while $\pm 15 \%$ of patients are being over dosed, causing unnecessary toxicities. ${ }^{35,37,38,48-50}$ These numbers illustrate the significant proportion of patients being treated outside the therapeutic window in the absence of dose titration.

\section{Defined and consistent exposure-response relationships}

TDM is only rational if defined and consistent exposure-response relationships have been demonstrated for both efficacy and toxicity. For this purpose, exposure can be interpreted as minimum plasma concentration $\left(C_{\min }\right)$, maximum plasma concentration $\left(C_{\max }\right)$, or area under the plasma concentration-time curve $(A \cup C)$. Extensive reviews summarizing the available literature on exposure-response relationships for each specific oral targeted therapy have previously been published. ${ }^{5-9}$ For many of these drugs exposure-response relationships have been demonstrated and pharmacokinetic targets could be identified (e.g. imatinib, pazopanib, and sunitinib ${ }^{51-54}$ ). For other drugs, pharmacokinetic targets based on exposure-efficacy analyses are not well established yet (e.g. dabrafenib, lenvatinib, and palbociclib ${ }^{5}$ ), but based on their mechanism of action, 
exposure-response relationships are to be expected. In these cases, the mean or median exposure could be taken as a reference. In previous analyses, we have demonstrated that targets based on exposure-efficacy analyses amounted to $82 \%( \pm 17 \%)$ and $85 \%( \pm 19 \%)$ of the average exposure in the population for kinase inhibitors and oral anti-hormonal drugs, respectively. ${ }^{5-7}$ Therefore, targeting the mean or median exposure generally leads to efficacious concentrations (as the real exposure-efficacy threshold is expected to be lower). The fact that the exposure-efficacy threshold is generally lower than the average exposure in the population is not a surprising finding, since the efficacy of these drugs has been proven in phase III trials indicating that the mean exposure should be sufficient to generate an antitumor response.

The magnitude of exposure-response relationships can be illustrated by pazopanib, for which a clear exposure-efficacy relationship exists, with progression-free survival (PFS) being significantly longer in patients with $C_{\min } \geq 20.5 \mathrm{mg} / \mathrm{L}$ (52.0 weeks versus 19.6 weeks ${ }^{53}$ ). The PFS of patients with an exposure below this target is even comparable with placebo (4.2 months $)^{55}$, making treatment at an inadequate pharmacokinetic exposure as ineffective as no treatment at all.

To overcome resistance, newer generation kinase inhibitors have been designed that block their target irreversibly (e.g. osimertinib, ibrutinib, and afatinib). ${ }^{56-58}$ It still has to be elucidated how this affects exposure-response relationships, but based on their irreversible mechanism of action, it could be expected that these agents are relatively overdosed due to the MTD paradigm currently still used in dose finding studies. Since these drugs bind their target covalently, inhibition endures even after the drug has been cleared from the systemic circulation. Therefore, the efficacy threshold could be lower than the mean pharmacokinetic exposure at the recommended dose. So far, for none of these agents clear exposure-response relationships have been identified. For example, for osimertinib, a retrospective analysis of 780 subjects showed no association between exposure and response. ${ }^{59}$

At the time new oral targeted therapies are approved, in most cases, insufficient data is available to draw conclusions on exposure-response relationships, while typically hundreds of patients have been treated with these drugs in the dose-finding and pivotal studies. However, data on pharmacokinetic exposure is often not structurally being collected in all patients. It is of great value to incorporate these pharmacokinetic analyses in the early stages of clinical development of these new drugs to ensure patients can be treated at a dose giving them adequate exposure.

Thus, for many oral targeted therapies defined and consistent exposure-response relationships exist, for others it can be reasonably expected while awaiting conclusive data, while for some exposure-response relationships might not be expected based on their irreversible mechanism of action and relatively high dose administered. 


\section{Feasible dose-adaptation strategy}

For drugs to be suitable candidates for TDM, feasible dose-adaptation strategies should exist, leading to target attainment without additional toxicities. Prospective clinical studies have already shown TDM to be feasible for pazopanib ${ }^{48,49}$, sunitinib ${ }^{48,50}$, imatinib ${ }^{48,60}$, everolimus $^{61}$, and endoxifen ${ }^{62}$. Table 2 provides a summary of the results of these studies. In clinical practice, the dose-adaptation strategies used in these prospective studies could be applied (i.e. the same pharmacokinetic target and dose levels could be used). Also, algorithms describing dose-adaptation schedules for other oral targeted therapies have been published previously. ${ }^{6}$

Figure 2 provides a schematic overview of pharmacokinetically guided dose individualization, in which pazopanib is used as an example. Patients start treatment at the standard fixed dose. At regular time intervals, pharmacokinetic sampling is performed (e.g. 4, 8, and 12 weeks after start of treatment, and every 12 weeks thereafter). In case of pharmacokinetic exposure below the predefined target and acceptable toxicities, the dosage can be increased with one dose level.

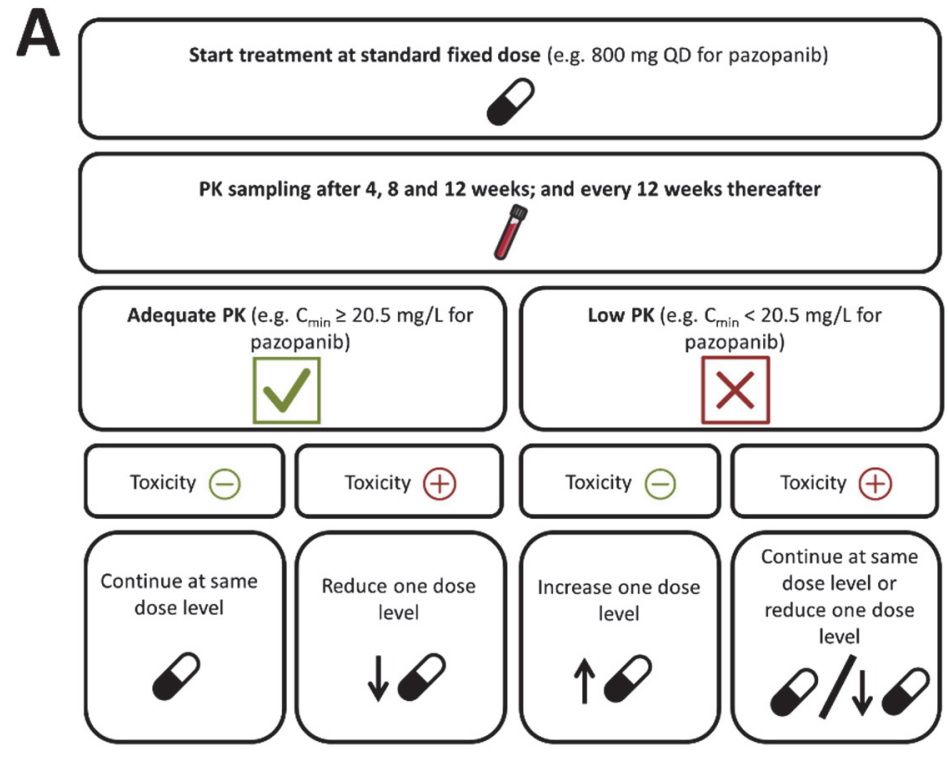

\begin{tabular}{|c|c|}
\hline Dose level & Pazopanib dose \\
\hline-3 & $200 \mathrm{mg}$ QD \\
\hline-2 & $400 \mathrm{mg}$ QD \\
\hline-1 & $600 \mathrm{mg} Q \mathrm{D}$ \\
\hline 0 & $800 \mathrm{mg}$ QD \\
\hline+1 & $400 \mathrm{mg}$ BID \\
\hline+2 & $400 \mathrm{mg}$ BID + food \\
\hline+3 & $400 \mathrm{mg} / 600 \mathrm{mg}+$ food \\
\hline+4 & $600 \mathrm{mg} \mathrm{BID}+$ food \\
\hline+5 & $600 \mathrm{mg} / 800 \mathrm{mg}+$ food \\
\hline+6 & $800 \mathrm{mg}$ BID + food \\
\hline+7 & $800 \mathrm{mg} / 1000 \mathrm{mg}+\mathrm{food}$ \\
\hline+8 & $1000 \mathrm{mg}$ BID + food \\
\hline
\end{tabular}

Figure 2 - Schematic overview of PK-guided dose adaptation strategy for oral anticancer drugs

A: Schematic overview of how therapeutic drug monitoring could be applied in clinical practice.

B: Example of proposed dose levels for pazopanib.

$B I D=$ twice daily; $P K=$ pharmacokinetics; $Q D=$ once daily

Dose-adaptation strategies should take into account the MTD of the drug, or - when the MTD has not been reached - the highest dose tested in phase I dose escalation trials, when deciding on the maximum dose level. Although it could be argued that pharmacokinetically guided dose escalation above the MTD could be safe as well (since this dose escalation will only be done in patients with a low pharmacokinetic exposure), this should only be considered with careful monitoring of side effects. 


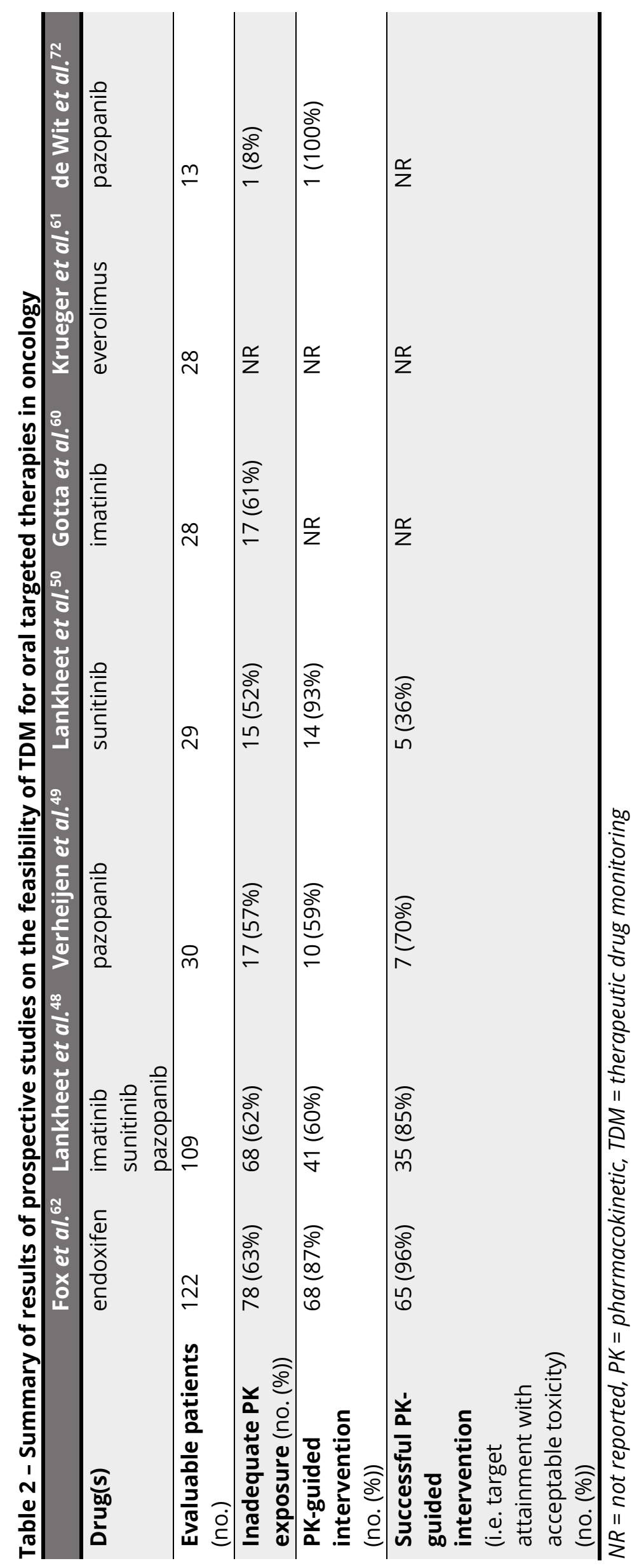


Pharmacokinetically guided interventions do not necessarily have to include absolute dose escalations, as for some oral targeted therapies, other options to increase pharmacokinetic exposure are available as well. For oral targeted therapies with a clinically significant food effect (e.g. abiraterone, lapatinib, and pazopanib), careful concomitant intake with food could be used as a first step in case of low pharmacokinetic exposure. ${ }^{3}$ Besides, for drugs with a saturable absorption profile (e.g. pazopanib and everolimus), splitting intake moments could provide a cost-neutral solution to attain adequate pharmacokinetic exposure. ${ }^{4,63}$

Another important consideration is the fact that progressive disease is irreversible. Therefore, it is important to attain an adequate pharmacokinetic exposure in each individual patient as soon as possible. In addition, it could be argued that dose reductions should only be made in case of intolerable toxicities, and not solely based on pharmacokinetic exposure (i.e. patients with high pharmacokinetic exposure, but without any side effects). On this aspect, TDM in oncology differs significantly from other disciplines, where it is often aimed at preventing toxicities as well due to its small therapeutic window.

To summarize, the feasibility of dose-adaptation strategies has been prospectively studied for several oral targeted therapies. ${ }^{48-50,60-62}$ All of these studies have shown TDM to be feasible, at least for a subset of patients. For other oral targeted therapies, possible dose-adaptation strategies have been described in literature or could be set up taking into account the mentioned considerations, while awaiting additional prospective studies. ${ }^{6}$

\section{DISCUSSION}

In this concise review article, we discussed the conditions that should be fulfilled for oral targeted therapies in oncology to be suitable candidates for TDM. Apart from some exceptions (e.g. osimertinib or cabozantinib), for most oral targeted therapies all of these requirements are met, providing a strong rationale for TDM.

A practical advantage is that most oral anticancer drugs are administered at a once or twice daily basis, making the timing of sampling more convenient compared with intermittent dosing (e.g. classical chemotherapy or immunotherapy). TDM targets are generally based on trough concentrations $\left(C_{\min }\right)$. While ideally trough samples would be drawn, this is not always possible in routine clinical practice. In this case, samples could be drawn at a random time point and $C_{\min }$ can be estimated using several algorithms like the method proposed by Wang et al. ${ }^{64}$ or by Bayesian forecasting. In addition, a number of powerful pharmacokinetic computer tools are available for this purpose. ${ }^{65}$

Even though convincing evidence supports dose individualization of oral targeted therapies, TDM is still being scarcely applied in daily clinical care. One of the reasons for this is that randomized controlled trials (RCTs), demonstrating the added value of TDM on 
clinical treatment outcomes, are lacking. However, it is highly unlikely that these RCTs could ever be performed. First, high numbers of patients would be needed, while most oral targeted therapies are indicated for rare tumor types or for a small subset of patients. For example, a randomized phase 3 study of TDM in patients with gastro-intestinal stromal tumours (GIST) treated with imatinib has been terminated prematurely due to slow accrual. ${ }^{66}$ Second, it is difficult to secure (sufficient) funding for these types of studies. Furthermore, it is questionable whether it is ethical to fail to perform dose adjustments for some patients, when clear exposure-response relationships exist. Only a few RCTs of fixed dosing versus PK-guided dosing have ever been completed in oncology, all with chemotherapy. ${ }^{67-70}$

Therefore, we are currently performing a large multi-center prospective study, in which we investigate the feasibility and efficacy of TDM for 23 different oral targeted therapies in more than 600 patients (www.trialregister.nl; NTR $6866^{71}$ ). Patients starting regular treatment with one of these drugs can be included in this study. For each drug, pharmacokinetic targets and dose levels have been defined and are described in the protocol. Pharmacokinetic sampling and dose adaptations are performed according to the strategy depicted in Figure 2. Primary outcome is to halve the proportion of patients with pharmacokinetic exposure below the target after 12 weeks (compared with historical data). Secondary outcomes are the safety, feasibility, and efficacy of pharmacokinetically guided dosing and physician adherence to the tailored treatment recommendations. If this study underscores the results of previous retrospective studies and prospective feasibility studies, this will further support the implementation of pharmacokinetically guided dose optimization as the new standard. ${ }^{48-50,60-62,72}$

As can be seen in Table 2, results of PK-guided dose individualization studies are currently not being reported in a uniform way, making mutual comparisons difficult. Therefore, we propose that future studies should at least report the following:

- Proportion of patients with low pharmacokinetic exposure;

- Proportion of patients in whom PK-guided interventions were applied;

- Reasons why these were not applied in other patients (e.g. toxicity, physician adherence);

- Proportion of patients in which PK-guided interventions were successful, thus in which adequate PK-exposure was attained without intolerable toxicities.

In this way, study results could be compared more easily and potentially be combined in a meta-analytical approach.

It is essential to convince treating physicians of the importance of TDM, as they need to implement the treatment recommendations into clinical practice. Unwillingness of treating physicians to follow these treatment recommendations was the main reason that 
a previous randomized controlled trial could not demonstrate the benefit of TDM for imatinib. 60

Apart from the apparent advantages of TDM in optimizing pharmacokinetic exposure to improve treatment outcomes, TDM could serve several other purposes as well. First, it could play a role in detecting nonadherence to therapy. This is especially important in the case of long-term therapy, as compliance drastically decreases over time (e.g. for tamoxifen adherence was only $50 \%$ after four years of therapy ${ }^{73}$ ). Second, TDM could be helpful in the management of drug-drug interactions, since pharmacokinetic exposure to many oral targeted therapies is affected by concomitant use of CYP3A4 inhibitors/inducers or gastric acid-suppressive agents. ${ }^{74}$ Last, measuring plasma drug concentrations could also support dose titration in patients with renal or hepatic impairment.

\section{CONCLUSION}

The pharmacokinetic characteristics of (most) oral targeted therapies in oncology support dose individualization by therapeutic drug monitoring. To realize the full potential of personalized medicine, we should not only treat each patient with the right drug, but also at the right dose. 


\section{REFERENCES}

1. van Brummelen EMJ, Huitema ADR, van Werkhoven E, Beijnen JH, Schellens JHM. The performance of model-based versus rule-based phase I clinical trials in oncology. J Pharmacokinet Pharmacodyn 2016; 43: 235-242.

2. Mathijssen RHJ, Sparreboom A, VerweijJ. Determining the optimal dose in the development of anticancer agents. Nat Rev Clin Oncol 2014; 11: 272-281.

3. Willemsen AEACB, Lubberman FJE, Tol J, Gerritsen WR, van Herpen CML, van Erp NP. Effect of food and acid-reducing agents on the absorption of oral targeted therapies in solid tumors. Drug Discov Today 2016; 21: 962-976.

4. Yu H, van Erp N, Bins S, Mathijssen RHJ, Schellens JHM, Beijnen JH et al. Development of a pharmacokinetic model to describe the complex pharmacokinetics of pazopanib in cancer patients. Clin Pharmacokinet 2017; 56: 293-303.

5. Verheijen RB, Yu H, Schellens JHM, Beijnen JH, Steeghs N, Huitema ADR. Practical recommendations for therapeutic drug monitoring of kinase inhibitors in oncology. Clin Pharmacol Ther 2017; 102: 765-776.

6. Yu H, Steeghs N, Nijenhuis CM, Schellens JHM, Beijnen JH, Huitema ADR. Practical guidelines for therapeutic drug monitoring of anticancer tyrosine kinase inhibitors: focus on the pharmacokinetic targets. Clin Pharmacokinet 2014; 53: 305-325.

7. Groenland SL, van Nuland M, Verheijen RB, Schellens JHM, Beijnen JH, Huitema ADR et al. Therapeutic drug monitoring of oral anti- hormonal drugs in oncology. Clin Pharmacokinet 2019; 58: 299-308.

8. Widmer N, Bardin C, Chatelut E, Paci A, Beijnen JH, Levêque D et al. Review of therapeutic drug monitoring of anticancer drugs part two - targeted therapies. Eur J Cancer 2014; 50: 2020-2036.

9. de Wit D, Guchelaar HJ, den Hartigh J, Gelderblom H, van Erp NP. Individualized dosing of tyrosine kinase inhibitors: are we there yet? Drug Discov Today 2015; 20: 18-36.

10. Mcleod HL. Therapeutic drug monitoring opportunities in cancer therapy. Pharmacol Ther 1997; 74: 39-54.

11. Galpin AJ, Evans WE. Therapeutic drug monitoring in cancer management. Clin Chem 1993; 39: $2419-2430$.

12. Massoni E, Zamboniz WC. Pharmacokinetic optimisation of cancer chemotherapy - effect on outcomes. Clin Pharmacokinet 1997; 32: 324-343.

13. Hon YY, Evans WE. Making TDM work to optimize cancer chemotherapy: a multidisciplinary team approach. Clin Chem 1998; 400: 388-400.

14. de Jonge ME, Huitema ADR, Schellens JHM, Rodenhuis S, Beijnen JH. Individualised cancer chemotherapy: strategies and performance of prospective studies on therapeutic drug monitoring with dose adaptation: a review. Clin Pharmacokinet 2005; 44: 147-173.

15. Rustin GJS, van der Burg MEL, Griffin CL, Guthrie D, Lamont A, Jayson GC et al. Early versus delayed treatment of relapsed ovarian cancer (MRC OV05/EORTC 55955): a randomised trial. Lancet 2010; 376: 1155-1163. 
16. Rini BI, Melichar B, Ueda T, Grünwald V, Fishman MN, Arranz JA et al. Axitinib with or without dose titration for first-line metastatic renal-cell carcinoma: a randomised double-blind phase 2 trial. Lancet Oncol 2013; 14: 1233-1242.

17. Steffens M, Paul T, Hichert V, Scholl C, Von Mallek D, Stelzer C et al. Dosing to rash? - the role of erlotinib metabolic ratio from patient serum in the search of predictive biomarkers for EGFR inhibitor-mediated skin rash. Eur J Cancer 2016; 55: 131-139.

18. Perez CA, Song H, Raez LE, Agulnik M, Grushko TA, Dekker A et al. Phase II study of gefitinib adaptive dose escalation to skin toxicity in recurrent or metastatic squamous cell carcinoma of the head and neck. Oral Oncol 2012; 48: 887-892.

19. Jabbour E, Kantarjian H, O'Brien S, Shan J, Quintas-Cardama A, Faderl S et al. The achievement of an early complete cytogenetic response is a major determinant for outcome in patients with early chronic phase chronic myeloid leukemia treated with tyrosine kinase inhibitors. Blood 2011; 118: 4541-4547.

20. Armstrong AJ, Saad F, Phung D, Dmuchowski C, Shore ND, Fizazi K et al. Clinical outcomes and survival surrogacy studies of prostate-specific antigen declines following enzalutamide in men with metastatic castration-resistant prostate cancer previously treated with docetaxel. Cancer 2017; 123: 2303-2311.

21. Food and Drug Administration. Center for Drug Evaluation and Research. Clinical Pharmacology and Biopharmaceutics Review Enzalutamide. 2012. Available from: https://www.accessdata.fda.gov/drugsatfda_docs/nda/2012/203415orig1s000ClinPharmR. pdf.

22. Summary of Product Characteristics - Tamoxifen. Available from: https://www.geneesmiddeleninformatiebank.nl/smpc/h11198_smpc.pdf.

23. Herbrink M, de Vries N, Rosing H, Huitema ADR, Nuijen B, Schellens JHM et al. Quantification of 11 therapeutic kinase inhibitors in human plasma for therapeutic drug monitoring using liquid chromatography coupled with tandem mass spectrometry. Ther Drug Monit 2016; 38: 649-656.

24. Herbrink M, de Vries N, Rosing H, Huitema ADR, Nuijen B, Schellens JHM et al. Development and validation of a liquid chromatography - tandem mass spectrometry analytical method for the therapeutic drug monitoring of eight novel anticancer drugs. Biomed Chromatogr 2018; 32: 1-9.

25. van Nuland M, Venekamp N, de Vries N, de Jong KAM, Rosing H, Beijnen JH. Development and validation of an UPLC-MS/MS method for the therapeutic drug monitoring of oral antihormonal drugs in oncology. J Chromatogr B 2019; 1106-1107: 26-34.

26. Jager NGL, Rosing H, Linn SC, Schellens JHM, Beijnen JH. Dried blood spot self-sampling at home for the individualization of tamoxifen treatment: a feasibility study. Ther Drug Monit 2015; 37: 833-836.

27. Verheijen RB, Bins S, Thijssen B, Rosing H, Nan L, Schellens JHM et al. Development and clinical validation of an LC-MS/MS method for the quantification of pazopanib in DBS. Bioanalysis 2016; 8: 123-134.

28. de Wit D, den Hartigh J, Gelderblom H, Qian Y, den Hollander M, Verheul H et al. Dried blood spot analysis for therapeutic drug monitoring of pazopanib. J Clin Pharmacol 2015; 55: $1344-1350$ 
29. Antunes MV, Raymundo S, Wagner SC, Mattevi VS, Vieira N, Leite R et al. DBS sampling in imatinib therapeutic drug monitoring: from method development to clinical application. Bioanalysis 2015; 7: 2105-2117.

30. Verheijen RB, Thijssen B, Atrafi F, Schellens JHM, Rosing $\mathrm{H}$, de Vries $\mathrm{N}$ et al. Validation and clinical application of an LC-MS/MS method for the quantification of everolimus using volumetric absorptive microsampling. J Chromatogr B 2019; 1104: 234-239.

31. Willemsen AECAB, Knapen LM, de Beer YM, Brüggemann RJM, Croes S, van Herpen CML et al. Clinical validation study of dried blood spot for determining everolimus concentration in patients with cancer. EurJ Clin Pharmacol 2018; 74: 465-471.

32. Boons CCLM, Chahbouni A, Schimmel AM, Wilhelm AJ, den Hartog YM, Janssen JJWM et al. Dried blood spot sampling of nilotinib in patients with chronic myeloid leukaemia: a comparison with venous blood sampling. J Pharm Pharmacol 2017; 69: 1265-1274.

33. Nijenhuis CM, Huitema ADR, Marchetti S, Blank CU, Haanen JBAG, van Thienen JV et al. The use of dried blood spots for pharmacokinetic monitoring of vemurafenib treatment in melanoma patients. J Clin Pharmacol 2016; 56: 1307-1312.

34. Beumer JH, Kozo D, Harney RL, Caitlin N, Jarrah J, Christner SM et al. Automated imatinib immunoassay. Ther Drug Monit 2016; 37: 486-492.

35. Lankheet NAG, Knapen LM, Schellens JHM, Beijnen JH, Steeghs N, Huitema ADR. Plasma concentrations of tyrosine kinase inhibitors imatinib, erlotinib, and sunitinib in routine clinical outpatient cancer care. Ther Drug Monit 2014; 36: 326-34.

36. Verheijen RB, Swart LE, Beijnen JH, Schellens JHM, Huitema ADR, Steeghs N. Exposuresurvival analyses of pazopanib in renal cell carcinoma and soft tissue sarcoma patients: opportunities for dose optimization. Cancer Chemother Pharmacol 2017; 80: 1171-1178.

37. Carton E, Noe G, Huillard O, Golmard L, Giroux J, Cessot A et al. Relation between plasma trough concentration of abiraterone and prostate-specific antigen response in metastatic castration-resistant prostate cancer patients. Eur J Cancer 2017; 72: 54-61.

38. Nijenhuis CM, Huitema ADR, Blank CU, Haanen JBAG, van Thienen JV, Rosing $\mathrm{H}$ et al. Clinical pharmacokinetics of vemurafenib in BRAF-mutated melanoma patients. J Clin Pharmacol 2016; 57: 125-128.

39. Ouellet D, Gibiansky E, Leonowens C, O'Hagan A, Haney P, Switzky J et al. Population pharmacokinetics of dabrafenib, a BRAF inhibitor: effect of dose, time, covariates, and relationship with its metabolites. J Clin Pharmacol 2014; 54: 696-706.

40. Ouellet D, Kassir N, Chiu J, Mouksassi MS, Leonowens C, Cox D et al. Population pharmacokinetics and exposure-response of trametinib, a MEK inhibitor, in patients with BRAF V600 mutation-positive melanoma. Cancer Chemother Pharmacol 2016; 77: 807-817.

41. van Leeuwen RWF, van Gelder T, Mathijssen RHJ, Jansman FGA. Drug-drug interactions with tyrosine-kinase inhibitors: a clinical perspective. Lancet Oncol 2014; 15: e315-e326.

42. Chatelut E, Bruno R, Ratain MJ. Intraindividual pharmacokinetic variability: focus on smallmolecule kinase inhibitors. Clin Pharmacol Ther 2018; 103: 956-958.

43. Herbrink M, Nuijen B, Schellens JHM, Beijnen JH. Variability in bioavailability of small molecular tyrosine kinase inhibitors. Cancer Treat Rev 2015; 41: 412-422. 
44. Herbrink M, Nuijen B, Schellens JHM, Beijnen JH. High-tech drugs in creaky formulations. Pharm Res 2017; 34: 1751-1753.

45. Bullock JM, Rahman A, Liu Q. Lessons learned: dose selection of small molecule - targeted oncology drugs. Clin Cancer Res 2016; 22: 2630-2639.

46. Lacy S, Nielsen J, Yang B, Miles D, Nguyen L, Hutmacher M. Population exposure - response analysis of cabozantinib efficacy and safety endpoints in patients with renal cell carcinoma. Cancer Chemother Pharmacol 2018; 81: 1061-1070.

47. Hurwitz HI, Dowlati A, Saini S, Savage S, Suttle AB, Gibson DM et al. Phase I trial of pazopanib in patients with advanced cancer. Clin Cancer Res 2009; 15: 4220-4227.

48. Lankheet NAG, Desar IME, Mulder SF, Burger DM, Kweekel DM, van Herpen CML et al. Optimizing the dose in cancer patients treated with imatinib, sunitinib and pazopanib. $\mathrm{Br} J$ Clin Pharmacol 2017; 83: 2195-2204.

49. Verheijen RB, Bins S, Mathijssen RHJ, Lolkema MP, van Doorn L, Schellens JHM et al. Individualized pazopanib dosing: a prospective feasibility study in cancer patients. Clin Cancer Res 2016; 22: 5738-5746.

50. Lankheet NAG, Kloth JSL, Gadellaa-van Hooijdonk CGM, Cirkel GA, Mathijssen RHJ, Lolkema MPJK et al. Pharmacokinetically guided sunitinib dosing: a feasibility study in patients with advanced solid tumours. Br J Cancer 2014; 110: 2441-2449.

51. Larson RA, Druker BJ, Guilhot F, O'Brien SG, Riviere GJ, Krahnke $T$ et al. Imatinib pharmacokinetics and its correlation with response and safety in chronic-phase chronic myeloid leukemia: a subanalysis of the IRIS study. Blood 2008; 111: 4022-4028.

52. Demetri GD, Wang Y, Wehrle E, Racine A, Nikolova Z, Blanke CD et al. Imatinib plasma levels are correlated with clinical benefit in patients with unresectable/metastatic gastrointestinal stromal tumors. J Clin Oncol 2009; 27: 3141-3147.

53. Suttle AB, Ball HA, Molimard M, Hutson TE, Carpenter C, Rajagopalan D et al. Relationships between pazopanib exposure and clinical safety and efficacy in patients with advanced renal cell carcinoma. Br J Cancer 2014; 111: 1-8.

54. Houk BE, Bello CL, Poland B, Rosen LS, Demetri GD, Motzer RJ. Relationship between exposure to sunitinib and efficacy and tolerability endpoints in patients with cancer: results of a pharmacokinetic/pharmacodynamic meta-analysis. Cancer Chemother Pharmacol 2009; 66: 357-371.

55. Sternberg CN, Davis ID, Mardiak J, Szczylik C, Lee E, Wagstaff J et al. Pazopanib in locally advanced or metastatic renal cell carcinoma: results of a randomized phase III Trial. J Clin Oncol 2010; 28: 1061-1068.

56. Food and Drug Administration. Center for Drug Evaluation and Research. Clinical Pharmacology and Biopharmaceutics Review Osimertinib. 2015. Available from: http://www.accessdata.fda.gov/drugsatfda_docs/nda/2015/208434orig1s000ClinPharmR. pdf.

57. Food and Drug Administration. Center for Drug Evaluation and Research. Clinical Pharmacology and Biopharmaceutics Review Ibrutinib. 2013. Available from: https://www.accessdata.fda.gov/drugsatfda_docs/nda/2013/205552orig1s000ClinPharmR. pdf. 
58. Food and Drug Administration. Center for Drug Evaluation and Research. Clinical Pharmacology and Biopharmaceutics Review Afatinib. 2013. Available from: https://www.accessdata.fda.gov/drugsatfda_docs/nda/2013/201292orig1s000ClinPharmR. pdf.

59. Brown K, Comisar C, Witjes H, Maringwa J, De Greef R, Vishwanathan K et al. Population pharmacokinetics and exposure-response of osimertinib in patients with non-small cell lung cancer. BrJ Clin Pharmacol 2017; 83: 1216-1226.

60. Gotta V, Widmer N, Decosterd LA, Chalandon Y, Heim D, Gregor M et al. Clinical usefulness of therapeutic concentration monitoring for imatinib dosage individualization: results from a randomized controlled trial. Cancer Chemother Pharmacol 2014; 74: 1307-1319.

61. Krueger DA, Care MM, Holland K, Agricola K, Tudor C, Mangeshkar P et al. Everolimus for subependymal giant-cell astrocytomas in tuberous sclerosis. N Eng/J Med 2010; 363: 18011811.

62. Fox P, Balleine RL, Lee C, Gao B, Balakrishnar B, Menzies AM et al. Dose escalation of tamoxifen in patients with low endoxifen level: evidence for therapeutic drug monitoring the TADE study. Clin Cancer Res 2016; 22: 3164-3171.

63. Verheijen RB, Atrafi F, Schellens JHM, Beijnen JH, Huitema ADR, Mathijssen RHJ et al. Pharmacokinetic optimization of everolimus dosing in oncology: a randomized crossover trial. Clin Pharmacokinet 2018; 57: 637-644.

64. Wang Y, Chia Y, Nedelman J, Schran H, Mahon F, Molimard M. A therapeutic drug monitoring algorithm for refining the imatinib trough level obtained at different sampling times. Ther Drug Monit 2009; 31: 579-584.

65. Fuchs A, Csajka C, Thoma Y, Buclin T, Widmer N. Benchmarking therapeutic drug monitoring software: a review of available computer tools. Clin Pharmacokinet 2013; 52: 922.

66. Study of dose escalation versus no dose escalation of imatinib in metastatic gastrointestinal stromal tumors. Available from: http://clinicaltrials.gov/ct2/show/NCT01031628.

67. Evans W, Relling M, Rodman J, Crom W, Boyett J, Pui C. Conventional compared with individualized chemotherapy for childhood acute lymphoblastic leukemia. $N$ Engl J Med 1998; 338: 499-505.

68. Joerger M, Von Pawel J, Kraff S, Fisher J, Eberhardt W, Gauler T et al. Open-label, randomized study of individualized, pharmacokinetically (PK)-guided dosing of paclitaxel combined with carboplatin or cisplatin in patients with advanced non-small-cell lung cancer (NSCLC). Ann Oncol 2016; 27: 1895-1902.

69. Gamelin E, Delva R, Jacob J, Merrouche Y, Raoul JL, Pezet D et al. Individual fluorouracil dose adjustment based on pharmacokinetic follow-up compared with conventional dosage: results of a multicenter randomized trial of patients with metastatic colorectal cancer.J Clin Oncol 2008; 26: 2099-2105.

70. Fety R, Rolland F, Barberi-Heyob M, Hardouin A, Campion L, Conroy T et al. Clinical impact of pharmacokinetically-guided dose adaptation of 5-fluorouracil: results from a multicentric randomized trial in patients with locally advanced head and neck carcinomas. Clin Cancer Res 1998; 4: 2039-2045. 
71. Netherlands Trial Register. NL6695/NTR6866. Therapeutic drug monitoring for oral anticancer drugs. Available from: https://www.trialregister.nl/trial/6695.

72. de Wit D, van Erp NP, den Hartigh J, Wolterbeek R, Deursen MDH, Labots $M$ et al. Therapeutic drug monitoring to individualize the dosing of pazopanib: a pharmacokinetic feasibility study. Ther Drug Monit 2015; 37: 331-338.

73. Partridge AH, Wang PS, Winer EP, Avorn J. Nonadherence to adjuvant tamoxifen therapy in women with primary breast cancer. J Clin Oncol 2003; 21: 602-606.

74. Hussaarts KGAM, Veerman GDM, Jansman FGA, van Gelder T, Mathijssen RHJ, van Leeuwen RWF. Clinically relevant drug interactions with multikinase inhibitors: a review. Ther Adv Med Oncol 2019; 11: 1-34. 


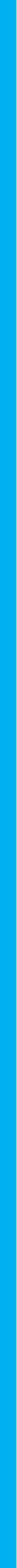





\section{PART II}

Exposure-response analyses 



\section{ABSTRACT}

\section{Background}

Abiraterone acetate is an oral 17a-hydroxylase/C17,20-lyase (CYP17) inhibitor approved for the treatment of metastatic castration-resistant prostate cancer (mCRPC) patients. Previously, a prospective observational trial demonstrated a relationship between abiraterone trough concentrations $\left(C_{\min }\right)$ in plasma and treatment efficacy. The aim of our study was to investigate the exposure-response relationship of abiraterone and its metabolites, and to study if the proposed target for abiraterone of $8.4 \mathrm{ng} / \mathrm{mL}$ is feasible in a "real-world" patient cohort.

\section{Patients and methods}

MCRPC patients who had at least one abiraterone plasma concentration at steady-state were included in this study. Plasma abiraterone and its metabolites levels were analyzed using a validated liquid chromatography-mass spectrometry method. Using calculated $C_{\min }$ values of abiraterone and its active metabolite $\Delta(4)$-abiraterone (D4A), univariate, and multivariable Cox regression analyses were performed.

\section{Results}

Sixty-two patients were included in this retrospective analysis, of which $42 \%$ were underexposed (mean abiraterone $C_{\min }<8.4 \mathrm{ng} / \mathrm{mL}$ ). In multivariable analysis, $C_{\min } \geq 8.4$ $\mathrm{ng} / \mathrm{mL}$ was associated with longer prostate-specific antigen (PSA) independent progression-free survival (16.9 vs. 6.1 months; $p=0.033$ ), which resulted in a hazard ratio of 0.44 (95\% confidence interval: $0.23-0.82, p=0.01$ ). $D 4 A C_{m i n}$ did not show a relationship with treatment efficacy.

\section{Conclusion}

Our study shows that $\mathrm{mCRPC}$ patients with an abiraterone $C_{\min } \geq 8.4 \mathrm{ng} / \mathrm{mL}$ have a better prognosis compared with patients with low $C_{\min }$. Monitoring $C_{\min }$ of abiraterone can help to identify those patients at risk of suboptimal treatment for whom treatment optimization may be appropriate. 


\section{INTRODUCTION}

Abiraterone is an inhibitor of 17a-hydroxylase/C17,20-lyase (CYP17), an enzyme involved in the intra- and extragonadal biosynthesis of androgens, including testosterone. Initially, abiraterone acetate was approved for treatment of metastatic castration-resistant prostate cancer (MCRPC) as it improves overall survival (OS) and progression-free survival (PFS) in this patient population compared with placebo. ${ }^{1,2}$

Following oral ingestion, abiraterone acetate is rapidly deacetylated to form the active substance abiraterone. Further metabolism into its major inactive metabolites abiraterone sulfate and abiraterone $\mathrm{N}$-oxide sulfate is facilitated by cytochrome P450 family 3A member 4 (CYP3A4) and sulfotransferase family 2A member 1 (SULT2A1). ${ }^{3}$ More recently, an active metabolite of abiraterone was discovered named $\Delta(4)$-abiraterone (D4A), which is formed by the enzyme 3ß-hydroxysteroid-dehydrogenase. ${ }^{4,5}$ D4A blocks CYP17, several steroidogenic enzymes, and the androgen receptor. ${ }^{5,6}$ Conversely, D4A is further metabolized to 3-keto-5-a-abiraterone, which stimulates the androgen receptor. ${ }^{7,8}$ The net result of these pharmacologic actions on therapeutic outcome remains to be elucidated.

Abiraterone acetate is administered in a fixed dose of $1000 \mathrm{mg}$ once daily (QD). Mean steady-state trough concentrations $\left(C_{\min }\right)$ at this approved dose are $11.1 \mathrm{ng} / \mathrm{mL}$ for abiraterone and $1.6 \mathrm{ng} / \mathrm{mL}$ for D4A., In a prospective observational trial, abiraterone $C_{\text {min }}$ has been associated with treatment response in MCRPC patients. In this study, plasma trough concentrations of abiraterone were significantly higher in prostate-specific antigen (PSA) responders $(n=38)$ compared with non-responders $(n=23)(12.0$ vs. $8.0 \mathrm{ng} / \mathrm{mL}$, $\mathrm{p}=0.0015) .{ }^{10}$ Furthermore, a threshold of $8.4 \mathrm{ng} / \mathrm{mL}$ has been identified, above which patients had a longer PFS compared with patients with $C_{\text {min }}$ below this target (12.2 vs. 7.4 months, $p=0.044) .{ }^{10}$ The same research group reported that higher $D 4 A C_{\min }$ is related to shorter OS, but not PFS ( $n=30)^{9}$

Abiraterone acetate has a large interpatient variability in $C_{\min }$ of $46 \% .^{10}$ Part of this variability may be accounted for by the food-effect, causing a sevenfold increase in maximum plasma concentration ( $\left.C_{\max }\right)$ with a low-fat meal and a 17-fold increase in $C_{\max }$ with a high-fat meal, compared with overnight fasting in healthy volunteers. ${ }^{11} \mathrm{~A}$ prospective clinical trial has shown that abiraterone acetate $250 \mathrm{mg}$ QD taken with a lowfat meal was non-inferior to abiraterone acetate at a standard dose of $1000 \mathrm{mg}$ QD in modified fasting state, in terms of PSA response and PFS ( $n=72) .{ }^{12}$ Furthermore, Stover et al. show that some men may benefit from taking abiraterone acetate concomitant with food. ${ }^{13}$

Previous studies clearly show an exposure-efficacy relationship between plasma trough concentrations of abiraterone and PFS. Yet, abiraterone acetate is still administered at 
fixed doses, which could lead to suboptimal treatment for some patients. Therapeutic drug monitoring (TDM), the clinical practice of measuring drug concentrations in biological fluids to individualize drug dosing, could be used to improve patient care. Based on the current data, TDM of abiraterone may be implemented with a $C_{\min }$ threshold of $8.4 \mathrm{ng} / \mathrm{mL}$. This threshold was established in a restrictive clinical study and needs to be confirmed with real-life data from daily clinical practice. The aim of our study was to assess the exposure-efficacy relationship of abiraterone and its major metabolites for the purpose of TDM in a "real-world" patient cohort. We hypothesized that patients with abiraterone $C_{\min } \geq 8.4 \mathrm{ng} / \mathrm{mL}$ will have a longer PFS compared with patients with a $C_{\min }<8.4 \mathrm{ng} / \mathrm{mL}$. A retrospective study was conducted to test this hypothesis.

\section{METHODS}

\section{Patients and sampling}

This was an observational study in the outpatient clinic of the Netherlands Cancer Institute-Antoni van Leeuwenhoek Hospital, Amsterdam. Abiraterone concentrations were monitored in all $\mathrm{MCRPC}$ patients using abiraterone acetate as part of routine clinical care. As authorized by the institute, data from clinical care were used retrospectively. Clinical characteristics were collected from medical records, including demographic data, medical history, abiraterone acetate dose, treatment duration, reason for discontinuation, concomitant medication, and PSA levels. Furthermore, testosterone and androstenedione concentrations were determined during treatment using a validated liquid chromatography-mass spectrometry (LC-MS/MS) assay. ${ }^{14}$

\section{Pharmacokinetics}

Blood samples were drawn as part of routine clinical care every 3 months on average. The date and time of blood withdrawal, and date and time of drug intake were recorded. Patients with at least one available abiraterone plasma concentration at steady-state were included in this study. Steady-state was considered to be reached after 1 week of treatment, taken into account the 15-hour half-life. ${ }^{3}$ Abiraterone and its metabolites D4A, abiraterone sulfate, and abiraterone $\mathrm{N}$-oxide sulfate were quantified using a validated LCMS/MS method. ${ }^{15,16}$ Plasma samples were collected at random time points during a dosing interval at routine patient visits to the outpatient clinic, and therefore, $C_{\text {min }}$ values were calculated from the measured concentrations. As abiraterone shows clear distribution pharmacokinetics, log-linear extrapolation was not feasible. Furthermore, the use of Bayesian estimates from a population pharmacokinetic model was considered, but this was complicated by high shrinkage. Therefore, we used the ratio of the observed concentration and median concentration as tool to calculate $C_{\text {min. }}$. First, we simulated a full population concentration-time curve of abiraterone with the pharmacokinetic model published by Stuyckens et al. ${ }^{17}$ Second, measured concentrations were divided by the 
simulated concentrations of the population curve at the recorded time points. Third, the ratio between measured concentrations and simulated concentrations was multiplied by the simulated $C_{\min }$ of the population curve to obtain the final calculated $C_{\min }$. Our data show that the shape of the D4A concentration-time curve is similar to that of abiraterone, and therefore, it is suggested that metabolite formation is rate-limiting in the clearance of D4A. As there is no pharmacokinetic model available for D4A, $C_{\min }$ was calculated in the same manner as the $C_{\min }$ of abiraterone. Measured concentrations of abiraterone sulfate and abiraterone $\mathrm{N}$-oxide sulfate were divided into three groups based on the time of sampling after dosing (TAD), being 0-4, 4-10, and 10-24 hours after drug intake. Samples taken before steady-state was reached or more than 24 hours after the last dose were excluded from further analysis.

\section{Outcome measures}

Three clinical end points regarding treatment response were evaluated separately in this study; PSA response, PSA independent PFS, and time to PSA progression (TTPP). PSA response was defined as $\geq 50 \%$ decrease in PSA from baseline, both according to the Prostate Cancer Working Group 2 (PCWG2) criteria. ${ }^{18,19}$ PSA independent PFS was defined as the time from treatment start to the first event of progression, being either radiographic progression, symptomatic progression (start of radiotherapy, samarium treatment, increase of analgesic dose, or a WHO performance level increase of at least 2), onset of next treatment or death from any cause. Radiographic progression was evaluated according to modified Response Evaluation Criteria in Solid Tumors (RECIST version 1.1). ${ }^{20}$ TTPP was defined as the time from treatment start to a $25 \%$ or greater PSA increase from the nadir, with an absolute increase in PSA levels of at least $2 \mathrm{ng} / \mathrm{mL}^{20}$, and had to be confirmed by a subsequent PSA value, also according to PCWG 2 criteria. Toxicity was defined as discontinuation due to adverse events, dose reductions due to adverse events or temporary treatment interruption.

\section{Statistics}

For the purpose of exposure-response analyses, the mean of all available abiraterone and metabolite levels per patient was used as parameter for exposure. The association between abiraterone plasma concentrations and metabolite concentrations was determined using the Spearman correlation test. Mann-Whitney $U$ tests were used for univariable analysis of PSA response and plasma concentrations of abiraterone and its metabolites. Using the abiraterone $C_{\min }$ target of $8.4 \mathrm{ng} / \mathrm{mL}$ as a cut-off value, patients were divided into two groups (adequate vs. Iow $C_{\text {min }}$ ) for PFS analyses. As no exposure target is known for D4A, D4A plasma concentrations were divided into quartiles for further analyses. PFS functions were estimated using the Kaplan-Meier method and predictive factors were assessed using the univariable model (log rank-test). A stepwise logistic regression was performed for the determination of a predictive score of PFS. Variables significantly associated with outcome in univariate analysis were used in the 
multivariate analysis. Ultimately, in multivariable analysis, PSA levels at baseline, WHO performance status, number of previous lines of treatment and whether patients switched from prednisone to dexamethasone during treatment were included as covariates. The following variables were tested but not included in the final model: age, weight, testosterone levels, androstenedione levels, prior treatment with docetaxel, hemoglobin, alkaline phosphatase, kidney, and liver function. All statistical analyses were performed in R (version 3.6.0, package "survival"). A post hoc power analysis was conducted to evaluate the statistical power of this study.

\section{RESULTS}

\section{Evaluable patients}

From June 2016 to June 2018, 62 patients on treatment with abiraterone acetate were included in this study. A full overview of patient characteristics is provided in Table 1. The median time of treatment was 13.6 months (range: 1.1-73.0 months). At data cut-off on 13 May 2019, 12 patients were still on abiraterone treatment. No relevant CYP-inhibiting or inducing co-medication was used during this treatment period. The Spearman correlation test showed that abiraterone and metabolite concentrations were statistically correlated, meaning that plasma samples with high abiraterone levels also contained high metabolite concentrations. Testosterone and androstenedione levels were below the lower limit of quantification of $0.01 \mathrm{ng} / \mathrm{mL}$ in all patients.

\section{Pharmacokinetics}

In total, 244 plasma samples were included. The distribution of time of sampling after dosing is shown in Supplementary Figure 1. Overall, a median (range) of 4(1-11) samples were available per patient. In aggregate, the median $\pm S D$ abiraterone $C_{\min }$ concentration was $9.3 \pm 10 \mathrm{ng} / \mathrm{mL}$, and median \pm SD metabolite plasma concentrations were $1.0 \pm 0.9$ $\mathrm{ng} / \mathrm{mL}$ for $\mathrm{D} 4 \mathrm{~A}, 8.7 \pm 7.2 \cdot 10^{3} \mathrm{ng} / \mathrm{mL}$ for abiraterone sulfate and $7.8 \pm 3.9 \cdot 10^{3} \mathrm{ng} / \mathrm{mL}$ for abiraterone $\mathrm{N}$-oxide sulfate. Interpatient variability (coefficient of variation; CV\%) of mean plasma concentrations at a $1000 \mathrm{mg}$ QD was 70\% for abiraterone and 61\% for D4A. Furthermore, mean intrapatient variability (CV\%) at a $1000 \mathrm{mg}$ QD was 53\% for abiraterone and $45 \%$ for D4A.

An overview of the distribution of mean abiraterone and $D 4 A C_{\text {min }}$ concentrations per patient is provided in Figure 1. Twenty-six (42\%) patients had an abiraterone $C_{\text {min }}$ below the target of $8.4 \mathrm{ng} / \mathrm{mL}$. Four patients received a dose reduction to $500 \mathrm{mg} Q \mathrm{QD}(\mathrm{n}=2)$ or $750 \mathrm{mg}$ QD $(\mathrm{n}=2)$ due to adverse events, including hepatotoxicity and fatigue. Two of these patients had an abiraterone $C_{\text {min }}$ below the target of $8.4 \mathrm{ng} / \mathrm{mL}$ after dose reduction. Of all explored clinical parameters, none were found to be significantly predictive of abiraterone plasma concentrations, except for body weight at baseline. Linear regression 
indicated that patients with a higher body weight at baseline had a lower plasma concentration $(p=0.014)$.

\section{Exposure-response analyses of abiraterone}

Among 62 included patients, 35 (56\%) patients were considered PSA responders, vs. 27 (44\%) patients without a PSA response. Figure 2 shows the relationship between $C_{\min }$ of abiraterone and PSA response. Mean plasma trough concentrations of abiraterone were $11.4 \mathrm{ng} / \mathrm{mL}$ in PSA responders compared with $7.2 \mathrm{ng} / \mathrm{mL}$ in non-responders $(p=0.18)$. The maximal change in PSA from baseline (\%) after start of treatment is shown for each patient in Figure 3. Plasma concentrations of the inactive metabolites abiraterone $\mathrm{N}$-oxide sulfate and abiraterone sulfate are depicted in Supplementary Figure 2. As no trough concentrations could be calculated for these metabolites, plasma levels are given in three groups based on the time after dosing. Median plasma concentrations were higher in PSA responders compared with non-responders in all groups but one.

Table 1 - Patient characteristics

\begin{tabular}{|c|c|c|c|}
\hline & & \multicolumn{2}{|c|}{ Abiraterone $C_{\min }$} \\
\hline & Total & $\geq 8.4 \mathrm{ng} / \mathrm{mL}$ & $<8.4 \mathrm{ng} / \mathrm{mL}$ \\
\hline Number of patients ( $\mathrm{n}(\%)$ ) & $62(100 \%)$ & $36(58 \%)$ & $26(42 \%)$ \\
\hline Age (mean, range) & $72(60-87)$ & $72(60-87)$ & $71(61-83)$ \\
\hline Weight (mean, range) & $89(57-175)$ & $91(57-175)$ & $85(68-117)$ \\
\hline \multicolumn{4}{|l|}{ WHO performance status (n (\%)) } \\
\hline 0 & $22(36 \%)$ & $12(33 \%)$ & $10(38 \%)$ \\
\hline 1 & $36(58 \%)$ & $22(61 \%)$ & $14(54 \%)$ \\
\hline 2 & $4(6 \%)$ & $2(5 \%)$ & $2(8 \%)$ \\
\hline Dose reduction $(\mathrm{n}(\%))$ & $4(6 \%)$ & $2(6 \%)$ & $2(8 \%)$ \\
\hline \multicolumn{4}{|l|}{ Previous lines of therapy (n (\%)) } \\
\hline 0 & $33(53 \%)$ & $23(64 \%)$ & $10(38 \%)$ \\
\hline 1 & $13(21 \%)$ & $7(19 \%)$ & $6(23 \%)$ \\
\hline 2 & $10(16 \%)$ & $3(8 \%)$ & $7(27 \%)$ \\
\hline 3 & $4(7 \%)$ & $2(5 \%)$ & $2(8 \%)$ \\
\hline 4 & $2(3 \%)$ & $1(3 \%)$ & $1(4 \%)$ \\
\hline Previous chemotherapy (n (\%)) & $26(42 \%)$ & $9(25 \%)$ & $17(65 \%)$ \\
\hline Switch to dexamethasone (n (\%)) & $33(53 \%)$ & $25(69 \%)$ & $8(69 \%)$ \\
\hline Number of samples $(n)$ & 244 & 165 & 79 \\
\hline Samples per patient (mean (range)) & $4(1-11)$ & $5(1-10)$ & $3(1-8)$ \\
\hline \multicolumn{4}{|l|}{ Median (range) $\mathbf{C}_{\min }(\mathrm{ng} / \mathrm{mL})$} \\
\hline Abiraterone & $9.3(2.0-49.8)$ & $14.9(8.5-49.8)$ & $6.3(2.0-8.4)$ \\
\hline $\mathrm{D} 4 \mathrm{~A}$ & $1.0(0.3-4.4)$ & $1.3(0.4-4.4)$ & $0.7(0.3-1.8)$ \\
\hline Median testosterone levels (ng/mL) & $<0.010^{a}$ & $<0.010^{a}$ & $<0.010^{a}$ \\
\hline Median androstenedione levels (ng/mL) & $<0.010^{a}$ & $<0.010^{a}$ & $<0.010^{a}$ \\
\hline
\end{tabular}

Demographic data and androgen levels are values at baseline.

${ }^{a}$ Data points below the lower limit of quantification of the bioanalytical method.

$D 4 A=\Delta(4)$-abiraterone 


\section{Mean calculated abiraterone Cmin}

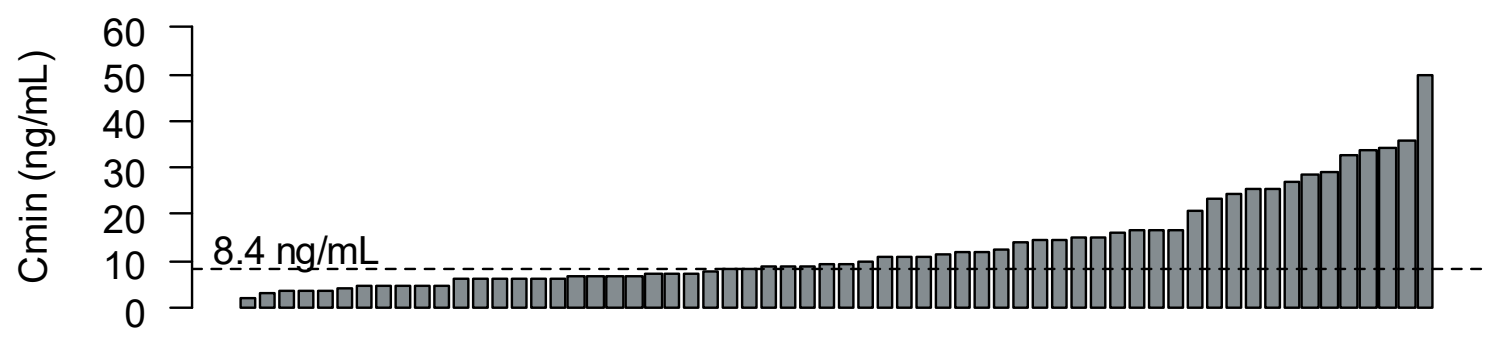

\section{Mean calculated D4A Cmin}

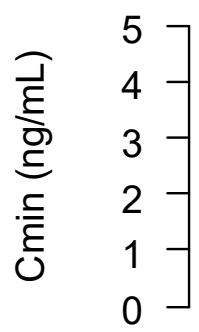

Figure 1 - Distribution of plasma concentrations of abiraterone and $\Delta$ (4)-abiraterone (D4A) in patients with metastatic castration-resistant prostate cancer (mCRPC), including the proposed target concentration for abiraterone of $8.4 \mathrm{ng} / \mathrm{mL}$

Each bar represents one patient.
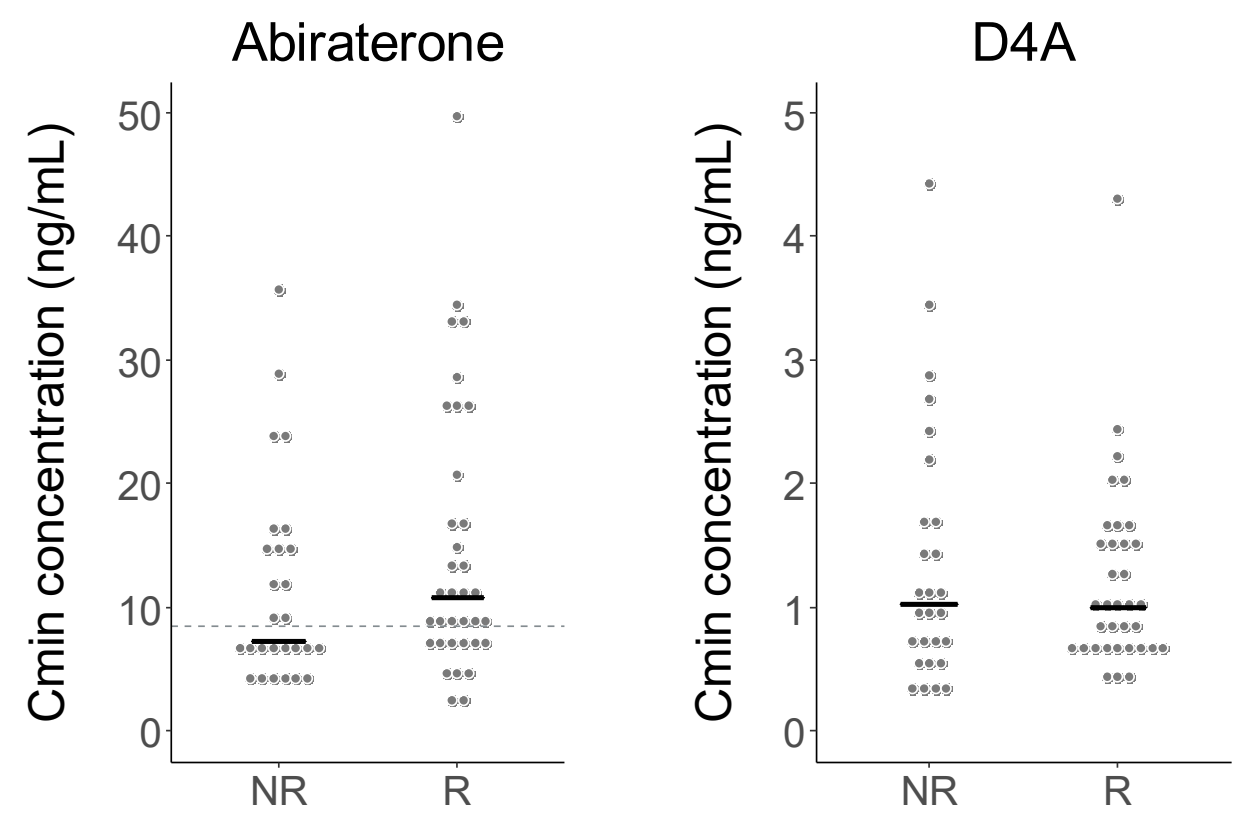

Figure 2 - Relationship between prostate-specific antigen response and the calculated trough concentration of abiraterone (left), $\Delta$ (4)-abiraterone (D4A) (right)

Horizontal lines represent the median concentration for PSA responders $(R, n=35)$ and nonresponders ( $N R, n=27)$, and the dotted lines represent the proposed target for abiraterone of 8.4 $\mathrm{ng} / \mathrm{mL}$. Mean plasma trough concentrations of abiraterone were $11.4 \mathrm{ng} / \mathrm{mL}$ in PSA responders compared with $7.2 \mathrm{ng} / \mathrm{mL}$ non-responders $(p=0.18)$ and D4A plasma concentrations were 1.0 $n g / m L$ in both PSA responders and non-responders $(p=0.88)$. 


\section{PSA change from baseline}

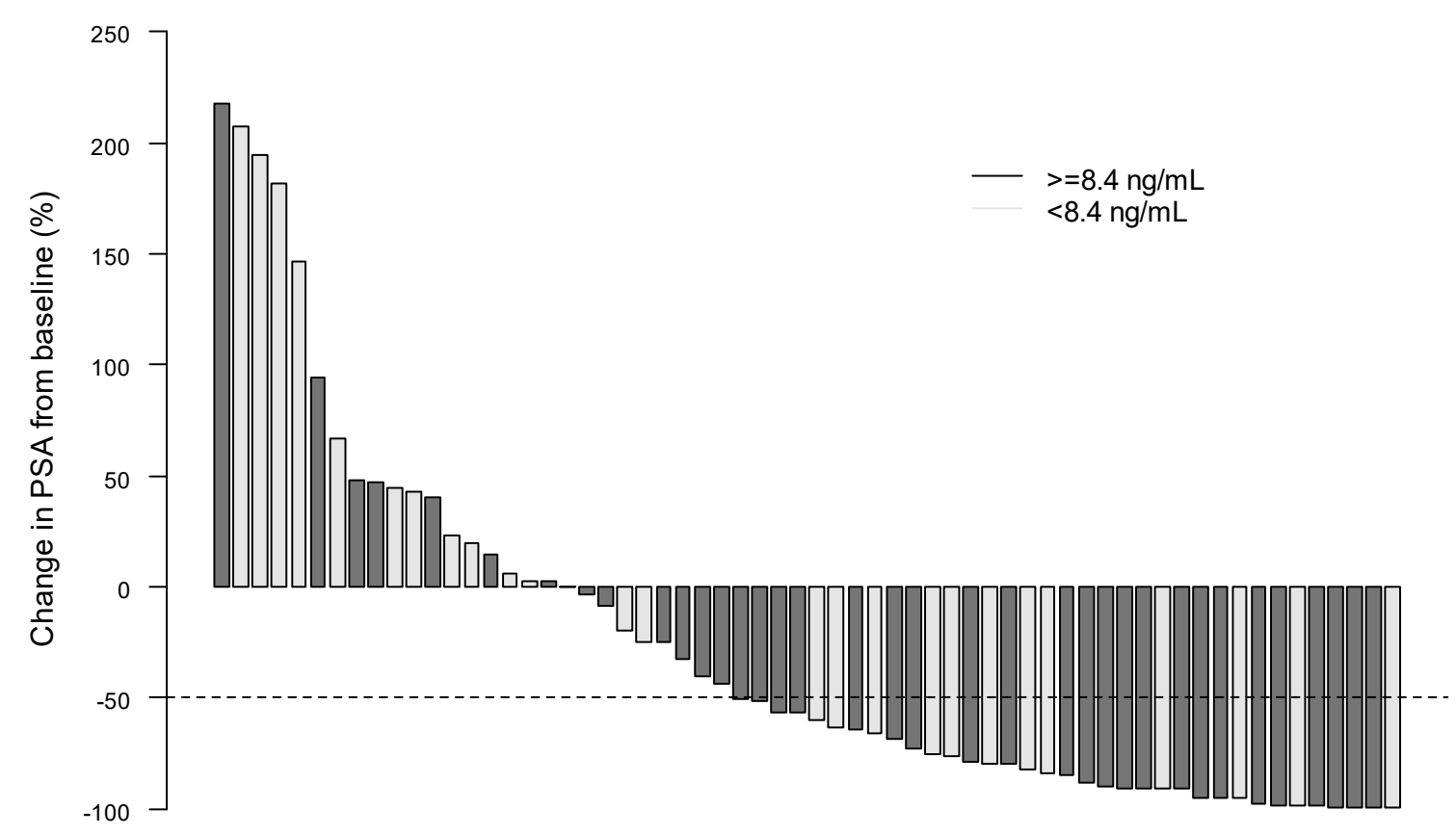

Figure 3 - Waterfall plot showing the PSA change from baseline (\%) after start of abiraterone acetate treatment

Each bar represents one patient and the colors indicate if this patient had an abiraterone $C_{\text {min }}$ above or below $8.4 \mathrm{ng} / \mathrm{mL}$. The dotted line indicates a 50\% PSA decrease from baseline, representing the cut-off for patients to be regarded PSA responders (> 50\%) or non-responders.

For PSA independent PFS, 62 patients were included with 50 events (81\% of patients) of progression. The remaining patients were still on treatment with abiraterone acetate. Median PSA independent PFS was 16.9 months in patients with an abiraterone $C_{\min } \geq 8.4$ $\mathrm{ng} / \mathrm{mL}$ compared with 6.1 months in patients with a $C_{\min }$ below the target $(p=0.077$, see Figure 4). The multivariable analysis resulted in a hazard ratio (HR) of $0.44(95 \% \mathrm{Cl} 0.23$ $0.82, p=0.01)$.

For TTPP analysis, 62 patients were included with 53 events ( $85 \%$ of patients) of PSA progression. Three patients were still on treatment, 1 patient died prior to PSA progression, and 5 patients did not show PSA progression but discontinued treatment due to radiographic progression. These patients were censored for TTPP analysis. Median TTPP in patients with an abiraterone $C_{\min } \geq 8.4 \mathrm{ng} / \mathrm{mL}$ was 19.8 months compared with 3.7 months in patients with a $C_{\min }$ below the target $(p=0.062$, see Figure 4). In multivariable analysis, $C_{\min } \geq 8.4 \mathrm{ng} / \mathrm{mL}$ resulted in a HR of 0.52 (95\% $\mathrm{Cl} 0.29-0.97, \mathrm{p}=0.038$ ).

A post hoc power analysis was conducted using the above described results. The power to detect a difference in PFS from 16.1 to 6.1 months (with a hazard ratio of 0.44 ) between patients with $C_{\min } \geq 8.4 \mathrm{ng} / \mathrm{mL}$ vs. $<8.4 \mathrm{ng} / \mathrm{mL}$, when there are 36 subjects in the first group and 26 in the second, using a two-sided log rank-test with alpha=0.05, was $80 \%$. 


\section{Exposure-response analyses of D4A}

Figure 2 shows the relationship between $C_{\min }$ of D4A and PSA response. Plasma concentrations were $1.0 \mathrm{ng} / \mathrm{mL}$ in both PSA responders and non-responders $(p=0.88)$.

Patients were divided into quartiles based on plasma concentrations of D4A, and PFS analyses were performed using these groups. There was no significant difference in the four quartiles regarding PSA independent PFS (7.7 vs. 22 vs. 13 vs. 11 months, $p=0.47$ ).
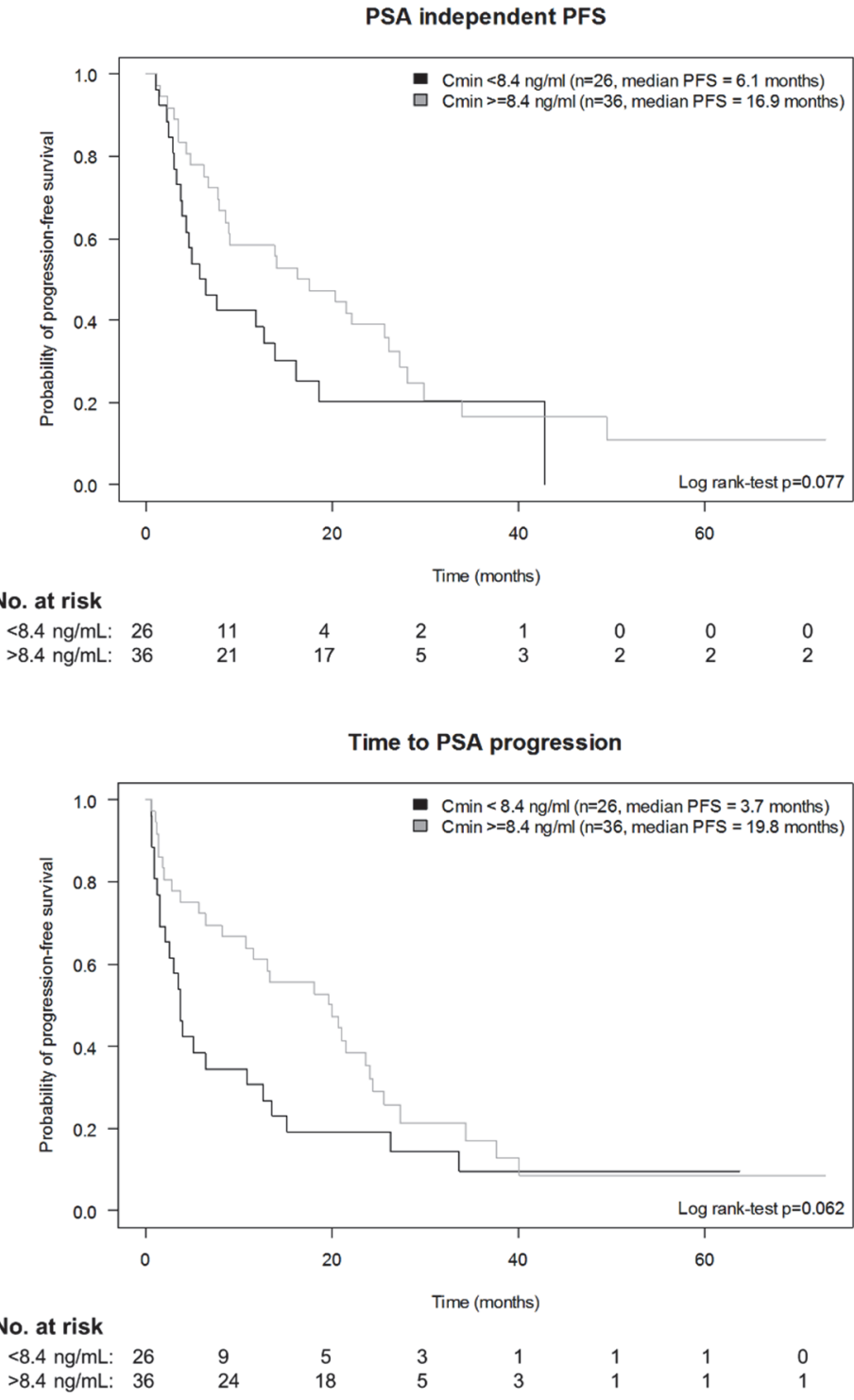

Figure 4 - Kaplan-Meier plots of PSA independent progression-free survival (PFS) in metastatic castration-resistant prostate cancer $(\mathrm{MCRPC})$ patients with a mean abiraterone $C_{\min }$ above ( $n=36$, grey line) or below ( $n=26$, black line) the exposure target of $8.4 \mathrm{ng} / \mathrm{mL}$

Prostate-specific antigen (PSA) independent PFS is shown in the upper panel, and time to PSA progression is shown in the lower panel. 
Furthermore, there was no significant difference in the four quartiles regarding TTPP (8.2 vs. 15 vs. 5.1 vs. 11 months, p=0.57). Kaplan-Meier curves are shown in Supplementary Figure 3. Both univariable and multivariable analysis did not support a relationship between D4A plasma concentrations and PFS.

\section{Exposure-toxicity analysis}

Of 62 included patients, four patients received a dose reduction and three patients temporarily discontinued treatment due to the presence of adverse events. Reasons for dose reduction or treatment interruption included fatigue, hepatotoxicity and abdominal pain. Median abiraterone $C_{\min }$ was $9.0 \mathrm{ng} / \mathrm{mL}$ for patients experiencing clinically relevant adverse events, compared with $9.3 \mathrm{ng} / \mathrm{mL}$ in those who did not $(p=1.0)$. Moreover, median D4A $C_{\min }$ concentrations were 1.1 vs. 1.0 for patients with and without adverse events, respectively $(p=0.60)$.

\section{DISCUSSION}

In this study, plasma concentrations of abiraterone and its metabolites were monitored in a clinical setting. To our knowledge, this is the first study to evaluate the correlation between abiraterone $C_{\min }$ and response in a real-world patient cohort, including D4A and other metabolite data. Obtaining real-life data is relevant for clinical practice, as this better reflects daily practice than data derived from clinical trials. ${ }^{21}$ Abiraterone acetate is administered at a fixed dose of $1000 \mathrm{mg}$ QD. Our data show that patients with an abiraterone $C_{\min } \geq 8.4 \mathrm{ng} / \mathrm{mL}$ have a longer PFS compared with patients with a pharmacokinetic exposure below this threshold. Furthermore, this study shows that $42 \%$ of patients with mCRPC may be underdosed with this standard fixed dosing regimen and could benefit from an individualized dosing strategy, which is in line with the previously reported $35 \%$ of patients having a $C_{\min }$ below the target. ${ }^{10}$

D4A was included in PFS analyses as it shows anti-androgen activity. However, it may be further converted to an androgen-stimulating metabolite and, therefore, the net contribution of D4A to the anti-tumor effect of abiraterone is ambiguous. ${ }^{7,8}$ Although a previous study has shown that a higher D4A $C_{\text {min }}$ was associated with shorter OS (HR 1.54, 95\% Cl 1.06-2.22, $\mathrm{p}=0.022$ ) but not with $\mathrm{PFS}^{9}$, our study did not reveal a relationship between D4A $C_{\min }$ and treatment response, PSA independent PFS or TTPP. Moreover, abiraterone and D4A concentrations are correlated, which indicates that abiraterone $C_{\text {min }}$ may serve as a proxy for the total antitumor effect of abiraterone and its metabolites.

The exposure target for abiraterone of $8.4 \mathrm{ng} / \mathrm{mL}$ was based on a prospective observational study. ${ }^{10}$ The CYP17 inhibitory concentrations 50\% (IC50) value of abiraterone is $0.07 \mathrm{ng} / \mathrm{mL}$. After correcting for plasma protein binding (99\%), a minimum concentration of $7.0 \mathrm{ng} / \mathrm{mL}$ should be reached to inhibit $50 \%$ of CYP17 in plasma. The exposure target is close to this corrected IC50 value, which biologically substantiates the 
threshold. Moreover, the CYP17 IC50 of D4A is $0.035 \mathrm{ng} / \mathrm{mL}$. Given a protein binding of $99 \%$, a minimum concentration of $3.5 \mathrm{ng} / \mathrm{mL}$ should be achieved to inhibit $50 \%$ of the CYP17 enzyme. ${ }^{4,5}$ Only three patients reached this threshold, which could explain why no association was found between D4A plasma levels and response in this population.

Although we believe our study provides relevant information on exposure-response of abiraterone in real-life patients, our analysis does have some limitations. First, in this study not actual $C_{\min }$ but calculated (from measured) plasma concentrations were used. Although actual $C_{\min }$ may be more accurate than calculated $C_{\min }$, the practical implementation of TDM is more feasible if samples can be drawn at random times during the dosing interval as it can be combined with routine visits to the outpatient clinic. Second, the extent of adherence to abiraterone acetate was not available due to the retrospective nature of this analysis. Although treating physicians provided instructions on drug intake and usage, this may be a potential source of variability in abiraterone $C_{\text {min. }}$.

Based on our study and previously published data, an exposure target for abiraterone of $8.4 \mathrm{ng} / \mathrm{mL}$ seems appropriate for TDM. Patients with a $C_{\min }$ below this target may be advised to take the drug concomitant with food, thereby avoiding expensive dose increments. A single-dose study of abiraterone in healthy volunteers has shown that the area under the plasma concentration-time curve (AUC) and $C_{\text {max }}$ increase 10- and 17-fold after intake with a high-fat meal, respectively, and sevenfold and fivefold after intake with a low-fat meal compared with overnight fasting, respectively. ${ }^{11}$ The same study showed a less pronounced effect in MCRPC patients when comparing a modified fasting state with food intake (similar exposure with low-fat meals and a twofold increase with high-fat meals). ${ }^{11}$ Furthermore, previous research has shown that some men may benefit from concomitant intake of abiraterone acetate with food in terms of PSA progression. ${ }^{13}$ This may be attributed to a lower percentage of patients with $C_{\min }<8.4 \mathrm{ng} / \mathrm{mL}$. Based on this information, concomitant intake of abiraterone with a low-fat meal may increase plasma levels up to fivefold, which would be sufficient for the majority of included patients with $C_{\min } \leq 8.4 \mathrm{ng} / \mathrm{mL}$ to reach plasma levels above the target. Treatment optimization by individualized dosing strategies could lead to better efficacy of abiraterone and higher treatment response. Furthermore, the lack of a relationship between exposure and toxicity suggests that increasing plasma levels will, in these ranges, not result in additional toxicity. Although more research is needed to confirm our findings and to further study the $8.4 \mathrm{ng} / \mathrm{mL}$ threshold, we advise clinicians to consider integrating TDM of abiraterone into standard treatment of MCRPC patients. Currently, a study is performed in our institute to investigate the feasibility of TDM with abiraterone using a food intervention ${ }^{22}$ by which we hope to improve outcome for MCRPC patients treated with abiraterone acetate. 


\section{CONCLUSION}

Our study shows that patients with an abiraterone trough level above $8.4 \mathrm{ng} / \mathrm{mL}$ have a longer PFS compared with patients with a pharmacokinetic exposure below this threshold. Exposure to the active metabolite D4A did not show a relationship with treatment efficacy and therefore may not add to the prognostic value of abiraterone plasma levels. Monitoring abiraterone $C_{\min }$ can identify those patients who are underdosed and we advise clinicians to consider integrating TDM of abiraterone into standard treatment of MCRPC patients. 


\section{REFERENCES}

1. de Bono JS, Logothetis CJ, Molina A, Fizazi K, North S, Chu L et al. Abiraterone and increased survival in metastatic prostate cancer. N Eng/ J Med 2011; 364: 1995-2005.

2. Ryan CJ, Smith MR, de Bono JS, Molina A, Logothetis CJ, de Souza P et al. Abiraterone in metastatic prostate cancer without previous chemotherapy. N Engl J Med 2013; 368: 138148.

3. US Food and Drug Administration (FDA). Clinical Pharmacology and Biopharmaceutics Review: Zytiga (abiraterone acetate). Availble from: https://www.accessdata.fda.gov/drugsatfda_docs/nda/2011/202379orig1s000clinpharmr.p df.

4. Li Z, Alyamani M, Li J, Rogacki K, Abazeed M, Upadhyay SK et al. Redirecting abiraterone metabolism to fine-tune prostate cancer anti-androgen therapy. Nature 2016; 533: 547-551.

5. Li Z, Bishop AC, Alyamani M, Garcia JA, Dreicer R, Bunch D et al. Conversion of abiraterone to D4A drives anti-tumour activity in prostate cancer. Nature 2015; 523: 347-351.

6. Emamekhoo H, Li Z, Sharifi N. Clinical significance of D4A in prostate cancer therapy with abiraterone. Cell cycle 2015; 14: 3213-3214.

7. Hettel D, Sharifi N. HSD3B1 status as a biomarker of androgen deprivation resistance and implications for prostate cancer. Nat Rev Urol 2018; 15: 191-196.

8. Alyamani M, Emamekhoo H, Park S, Taylor J, Almassi N, Upadhyay S et al. HSD3B1(1245A>C) variant regulates dueling abiraterone metabolite effects in prostate cancer. J Clin Invest 2018; 128: 3333-3340.

9. Blanchet B, Carton E, Alyamani M, Golmard L, Huillard O, Thomas-Scheomann A et al. A PK/PD study of Delta-4 abiraterone metabolite in metastatic castration-resistant prostate cancer patients. Pharmacol Res 2018; 136: 56-61.

10. Carton E, Noe G, Huillard O, Golmard L, Giroux J, Cessot A et al. Relation between plasma trough concentration of abiraterone and prostate-specific antigen response in metastatic castration-resistant prostate cancer patients. Eur J Cancer 2017; 72: 54-61.

11. Chi KN, Spratlin J, Kollmannsberger C, North S, Pankras C, Gonzalez M et al. Food effects on abiraterone pharmacokinetics in healthy subjects and patients with metastatic castrationresistant prostate cancer. J Clin Pharmacol 2015; 55: 1406-1414.

12. Szmulewitz RZ, Peer C, Ibraheem A, Martinez E, Kozloff MF, Carthon B et al. Prospective international randomized phase II study of low-dose abiraterone with food versus standard dose abiraterone in castration-resistant prostate cancer. J Clin Oncol 2018; 36: 1389-1395.

13. Stover JT, Moore RA, Davis K, Harrison MR, Armstrong AJ. Reversal of PSA progression on abiraterone acetate through the administration with food in men with metastatic castrationresistant prostate cancer. Prostate Cancer Prostatic Dis 2015; 18: 161-166.

14. van Nuland M, Venekamp N, Wouters WME, van Rossum HH, Rosing H, Beijnen JH. LCMS/MS assay for the quantification of testosterone, dihydrotestosterone, androstenedione, cortisol and prednisone in plasma from castrated prostate cancer patients treated with abiraterone acetate or enzalutamide. J Pharm Biomed Anal 2019; 170: 161-168.

15. van Nuland $M$, Hillebrand MJ, Rosing $H$, Schellens JHM, Beijnen JH. Development and 
validation of an LC-MS/MS method for the simultaneous quantification of abiraterone, enzalutamide, and their major metabolites in human plasma. Ther Drug Monit 2017; 39: 243251.

16. van Nuland M, Rosing $\mathrm{H}$, de Vries J, Ovaa H, Schellens JHM, Beijnen JH. An LC-MS/MS method for quantification of the active abiraterone metabolite $\Delta(4)$-abiraterone (D4A) in human plasma.J Chromatogr B Anal Technol Biomed Life Sci 2017; 1068-1069: 119-124.

17. Stuyckens K, Saad F, Xu XS, Ryan CJ, Smith MR, Griffin TW et al. Population pharmacokinetic analysis of abiraterone in chemotherapy-naive and docetaxel-treated patients with metastatic castration-resistant prostate cancer. Clin Pharmacokinet 2014; 53: 1149-1160.

18. Scher HI, Morris MJ, Stadler WM, Higano CS, Halabi S, Smith MR et al. The Prostate Cancer Working Group 3 (PCWG3) consensus for trials in castration-resistant prostate cancer (CRPC). J Clin Oncol 2015; 33: 5000.

19. Scher HI, Halabi S, Tannock I, Morris M, Sternberg CN, Carducci MA et al. Design and end points of clinical trials for patients with progressive prostate cancer and castrate levels of testosterone: recommendations of the Prostate Cancer Clinical Trials Working Group. J Clin Oncol 2008; 26: 1148-1159.

20. Therasse P, Arbuck SG, Eisenhauer EA, Wanders J, Kaplan RS, Rubinstein L et al. New guidelines to evaluate the response to treatment in solid tumors. JNCIJ Natl Cancer Inst 2000; 92: 205-216.

21. Westgeest HM, Uyl-de Groot CA, van Moorselaar RJA, de Wit R, van den Bergh ACM, Coenen JLLM et al. Differences in trial and real-world populations in the dutch castration-resistant prostate cancer registry. Eur Urol Focus 2018; 4: 694-701.

22. Groenland SL, van Eerden RAG, Verheijen RB, Koolen SLW, Moes DJAR, Desar IME et al. Therapeutic drug monitoring of oral anticancer drugs: the DPOG-TDM protocol for a prospective study. Ther Drug Monit 2019; 41: 561-567. 


\section{SUPPLEMENTARY DATA}

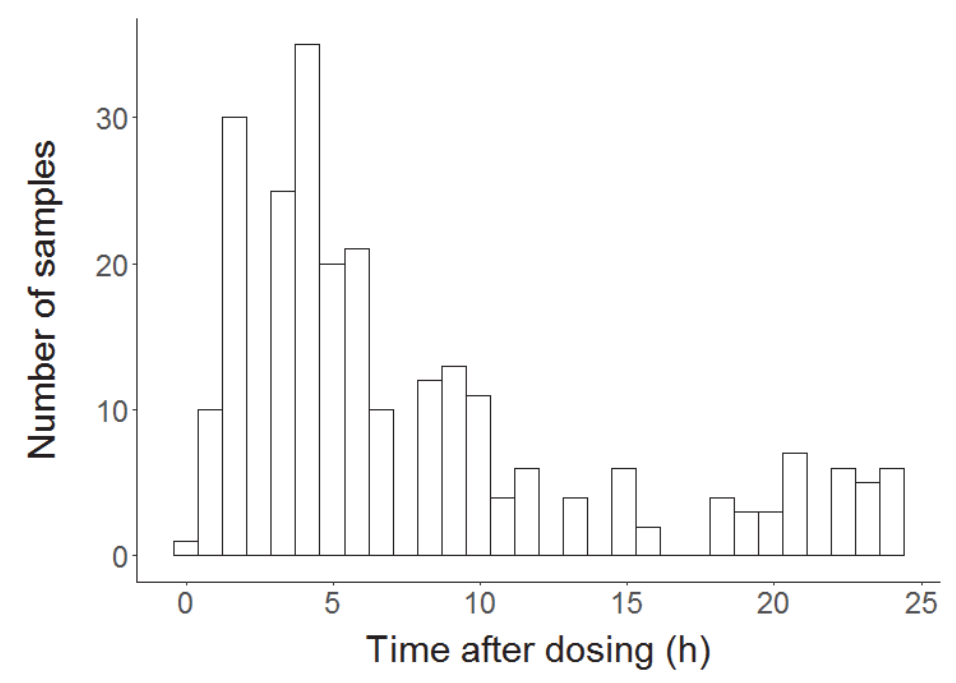

Supplementary Figure 1 - Histogram showing the number of samples taken at a certain time after dosing (TAD)
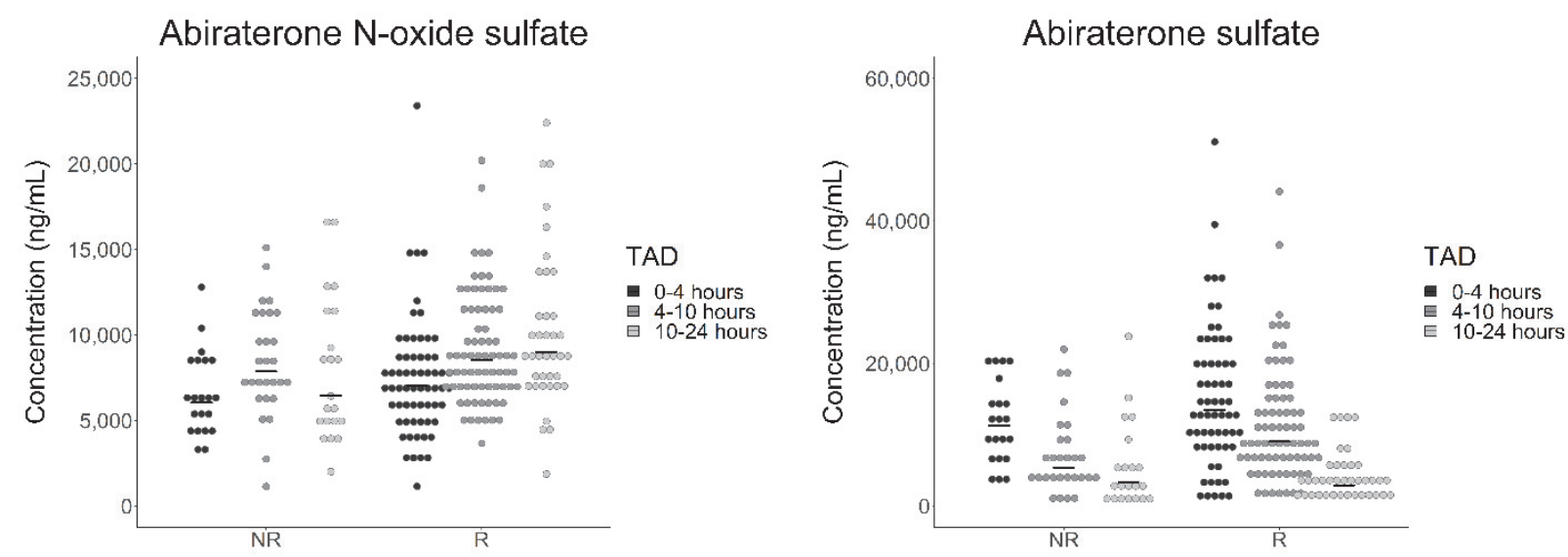

Supplementary Figure 2 - Relationship between prostate-specific antigen response and plasma concentrations of abiraterone $\mathrm{N}$-oxide sulfate (left) and abiraterone sulfate (right) Plasma concentrations are divided into three groups, based on the time of sampling after dosing (TAD), being 0-4, 4-10, and 10-24 hours. Horizontal lines represent the median concentration for PSA responders (R) and non-responders (NR). Median plasma concentrations of abiraterone $\mathrm{N}$ oxide sulfate per group were $6.0 \cdot 10^{3}, 7.9 \cdot 10^{3}$ and $6.4 \cdot 10^{3} \mathrm{ng} / \mathrm{mL}$ for the non-responders vs. $7.0 \cdot 10^{3}$, $8.5 \cdot 10^{3}$ and $9.0 \cdot 10^{3} \mathrm{ng} / \mathrm{mL}$ for PSA responders, respectively. Median plasma concentrations of abiraterone sulfate were $11 \cdot 10^{3}, 5.4 \cdot 10^{3}$ and $3.4 \cdot 10^{3} \mathrm{ng} / \mathrm{mL}$ vs. $14 \cdot 10^{3}, 9.1 \cdot 10^{3}$ and $2.9 \cdot 10^{3} \mathrm{ng} / \mathrm{mL}$ per group, respectively. 
PSA independent PFS

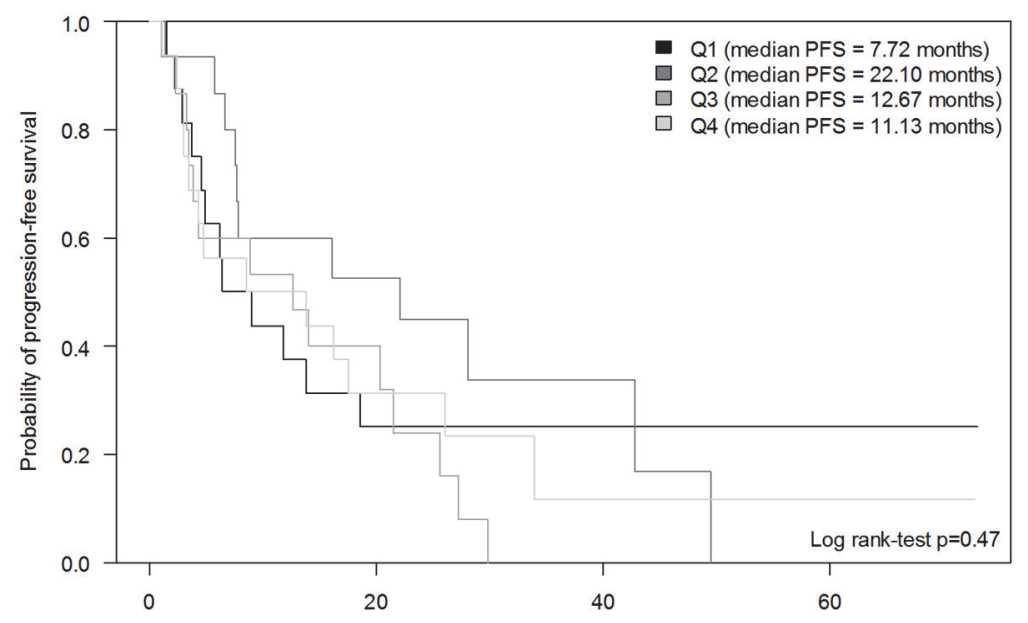

\begin{tabular}{lllllllll} 
No. at risk & \multicolumn{7}{c}{ Time (months) } \\
Q1: & 16 & 7 & 4 & 2 & 1 & 1 & 1 & 1 \\
Q2: & 15 & 9 & 7 & 3 & 2 & 0 & 0 & 0 \\
Q3: & 15 & 8 & 5 & 0 & 0 & 0 & 0 & 0 \\
Q4: & 16 & 8 & 5 & 2 & 1 & 1 & 1 & 1
\end{tabular}

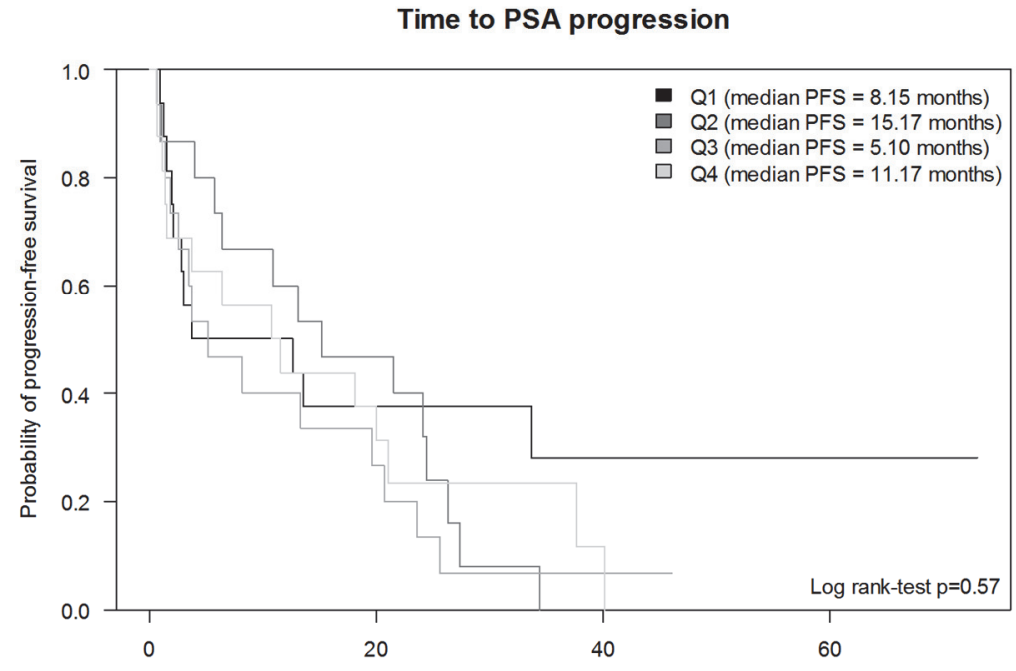

No. at risk

$\begin{array}{lcccccccc}\text { Q1: } & 16 & 8 & 6 & 4 & 2 & 2 & 2 & 1 \\ \text { Q2: } & 15 & 10 & 7 & 1 & 0 & 0 & 0 & 0 \\ \text { Q3: } & 15 & 6 & 4 & 1 & 1 & 0 & 0 & 0 \\ \text { Q4: } & 16 & 9 & 6 & 2 & 1 & 0 & 0 & 0\end{array}$

Supplementary Figure 3 - Kaplan-Meier plots of PSA independent progression-free survival (PFS) in metastatic castration-resistant prostate cancer (MCRPC) patients for each quartile of $\Delta(4)$-abiraterone (D4A) concentrations

Prostate-specific antigen (PSA) independent PFS is shown in the upper panel, and time to PSA progression is shown in the lower panel. 



\section{ABSTRACT}

Crizotinib and alectinib are anaplastic lymphoma kinase (ALK)-inhibitors indicated for the treatment of ALK-positive metastatic non-small cell lung cancer (NSCLC). At the currently used fixed doses, interindividual variability in exposure is high. The aim of this study was to investigate whether minimum plasma concentrations $\left(C_{\min }\right)$ of crizotinib and alectinib are related to efficacy and toxicity. An observational study was performed, in which ALKpositive NSCLC patients who were treated with crizotinib and alectinib and from whom pharmacokinetic samples were collected in routine care, were included in the study. Exposure-response analyses were performed using previously proposed $C_{\min }$ thresholds of $235 \mathrm{ng} / \mathrm{mL}$ for crizotinib and $435 \mathrm{ng} / \mathrm{mL}$ for alectinib. Forty-eight crizotinib and 52 alectinib patients were included. For crizotinib, median progression-free survival (mPFS) was 5.7 vs. 17.4 months for patients with $C_{\min }<235 \mathrm{ng} / \mathrm{mL}(48 \%)$ and $\geq 235 \mathrm{ng} / \mathrm{mL}$, respectively $\left(p=0.08\right.$ ). In multivariable analysis, $C_{\min }<235 \mathrm{ng} / \mathrm{mL}$ resulted in a hazard ratio (HR) of 1.79 (95\% confidence interval $(\mathrm{Cl}) 0.90-3.59, \mathrm{p}=0.100)$. In a pooled analysis of all crizotinib patients (not only ALK-positive, $n=79$ ), the HR was 2.15 (95\% Cl 1.21-3.84, $\mathrm{p}=0.009$ ). For alectinib, mPFS was 12.6 months vs. not estimable $(95 \% \mathrm{Cl}$ 19.8-not estimable) for patients with $C_{\min }<435 \mathrm{ng} / \mathrm{mL}(37 \%)$ and $\geq 435 \mathrm{ng} / \mathrm{mL}$, respectively $(p=0.04)$. Multivariable analysis resulted in an HR of $4.29(95 \% \mathrm{Cl} 1.33-13.90, \mathrm{p}=0.015)$. In conclusion, PFS of crizotinib and alectinib treated NSCLC patients is prolonged in patients with $C_{\min } \geq$ $235 \mathrm{ng} / \mathrm{mL}$ and $435 \mathrm{ng} / \mathrm{mL}$, respectively. Therefore, therapeutic drug monitoring should be part of routine clinical management for these agents. 


\section{INTRODUCTION}

Anaplastic lymphoma kinase (ALK) rearrangements are identified as oncogenic drivers in $3-7 \%$ of non-small cell lung cancer (NSCLC) patients. ${ }^{1-3}$ Over the past decade, several ALK tyrosine kinase inhibitors have become available and have resulted in significant improvements in overall survival, which is now extended to a median of close to four years in the metastatic setting. ${ }^{4}$ Crizotinib and alectinib are first and second generation ALKinhibitors, respectively, with alectinib being inherently more effective. ${ }^{5}$

Pharmacokinetic exposure to crizotinib and alectinib may be related to toxicity and efficacy. Currently, both crizotinib and alectinib are administered as oral fixed doses of $250 \mathrm{mg}$ twice daily (BID) and $600 \mathrm{mg}$ BID, respectively, exhibiting a high interindividual variability in pharmacokinetic exposure of $40-45 \% .6,7$ Nevertheless, for both crizotinib and alectinib, no relation between exposure and toxicity could be established based on the currently available data. ${ }^{6,7}$ However, in the registration study of crizotinib $(n=114)$ it was shown that patients with a minimum plasma concentration $\left(C_{\min }\right)$ in the lowest quartile (i.e. $<235 \mathrm{ng} / \mathrm{mL}$ ) had a significantly lower objective response rate and shorter progression-free survival (PFS) as compared with the upper three quartiles. ${ }^{6}$ Similarly, it has been shown that patients treated with alectinib $(n=46)$ in the lowest tertile of $C_{\text {min }}$ (i.e. $<435 \mathrm{ng} / \mathrm{mL}$ ) had less reduction in tumor size compared with the upper two tertiles. ${ }^{7}$

Therefore, personalized dosing based on measured drug levels (i.e. therapeutic drug monitoring) may be more rational, as a subgroup of patients might benefit from a higher dose of both crizotinib and alectinib.

However, before deciding whether individualized dosing might be appropriate, exposureefficacy relationships need to be confirmed in an independent patient cohort. Furthermore, clinical trial populations differ from real-life patients in several aspects. ${ }^{8}$ Therefore, exposure-efficacy analyses should preferably be performed in real-life patients, instead of in highly selected patients in clinical trials. For this reason, the aim of the observational study reported here was to investigate whether pharmacokinetic exposure to crizotinib and alectinib is related to efficacy and toxicity in a real-life patient cohort.

\section{METHODS}

\section{Patient population and data collection}

A retrospective observational cohort study was performed at The Netherlands Cancer Institute, Amsterdam, The Netherlands. Consecutive patients with ALK-positive NSCLC who were treated with crizotinib or alectinib were included. Pharmacokinetic (PK) samples of these patients were collected as part of routine care. An additional pooled analysis with all patients treated with crizotinib was performed (i.e. ALK-positive, c-ros oncogene 1 
(ROS1)-positive and mesenchymal epithelial transition growth factor (cMET) dysregulation). Of these patients, demographic data, prior lines of treatment, crizotinib and alectinib dose, treatment duration, reason for discontinuation, clinically relevant toxicities, and progression-free survival were retrospectively collected from medical records. Following initiation of treatment, imaging was performed twice every 6 weeks, thereafter every 12 weeks.

\section{Pharmacokinetic exposure}

Plasma samples were collected during routine follow-up visits to the outpatient clinic. Date and time of last drug intake and plasma sampling were recorded in order to calculate the time after dose. Crizotinib and alectinib concentrations were quantified using validated liquid chromatography-tandem mass spectrometry assays. ${ }^{9,10}$ As samples were collected at random time points during the dosing interval, $C_{\min }$ was calculated using the following algorithm ${ }^{11}$ :

$$
C_{\text {min }}=C_{\text {measured }} * 0.5^{\frac{\text { dosing interval-TAD }}{t_{1 / 2}}}
$$

in which $C_{\min }$ is the calculated minimum plasma concentration, $C_{\text {measured }}$ is the measured plasma concentration, dosing interval is the time between two consecutive administrations of the drug (i.e. 12 hours for crizotinib and alectinib), TAD is the time after dose (i.e. the time between last intake of the drug and collection of the PK sample), and $t_{1 / 2}$ is the elimination half-life of the drug (i.e. 42 hours for crizotinib ${ }^{6}$ and 32 hours for alectinib ${ }^{7}$ ). As crizotinib and alectinib have a longer elimination half-life than imatinib (i.e. 18 hours ${ }^{12}$ ), this algorithm should perform at least similarly to imatinib.

Samples drawn before steady-state was reached or more than one $t_{1 / 2}$ after the last dose were excluded from the analysis. The median of all available $C_{\min }$ levels per patient was taken as a measure of pharmacokinetic exposure.

\section{Exposure-response analyses}

Exposure-efficacy analyses were performed using previously proposed thresholds of 235 $\mathrm{ng} / \mathrm{mL}$ for crizotinib and $435 \mathrm{ng} / \mathrm{mL}$ for alectinib. ${ }^{6,7,13}$ PFS of patients with a median $C_{\min }$ above and below these thresholds was compared using univariable and multivariable Cox regression analyses. A two-sided $\mathrm{p}$-value $<0.05$ was considered statistically significant. Statistical analyses were performed using R version 3.3.6 (R Project, Vienna, Austria). ${ }^{14}$

Exposure-toxicity analyses were performed by comparing median $C_{\text {min }}$ between patients with and without clinically relevant toxicities, which was defined as toxicities leading to treatment interruption, dose reduction, or treatment discontinuation.

Median follow-up time was determined using the reverse Kaplan-Meier method. ${ }^{15}$ 


\section{Ethical regulations}

The institutional review board authorized the study on 15 November 2018. The need for written informed consent was waived.

\section{RESULTS}

\section{Patient characteristics}

In total, 100 consecutive patients were included (48 treated with crizotinib and 52 treated with alectinib) between 2012 and 2019. Baseline characteristics of these patients are provided in Table 1 and $\mathbf{2}$ for crizotinib and alectinib, respectively. At the time of data cutoff (26 April 2019), 11 and 34 patients were still on treatment for crizotinib and alectinib, respectively. For crizotinib, 23 patients (48\%) had a median $\mathrm{C}_{\min }<235 \mathrm{ng} / \mathrm{mL}$. For alectinib, 19 patients (37\%) had a median $\mathrm{C}_{\min }<435 \mathrm{ng} / \mathrm{mL}$.

Table 1 - Baseline characteristics of patients treated with crizotinib $(n=48)$

\begin{tabular}{|c|c|c|c|}
\hline Patient characteristic & $\begin{array}{l}\text { Median } C_{\min } \\
<235 \mathrm{ng} / \mathrm{mL} \\
(\mathrm{n}=23)\end{array}$ & $\begin{array}{l}\text { Median } C_{\min } \\
\geq 235 \mathrm{ng} / \mathrm{mL} \\
(\mathrm{n}=25)\end{array}$ & $\begin{array}{l}\text { All patients } \\
(n=48)\end{array}$ \\
\hline Gender, female & $9(39 \%)$ & $14(56 \%)$ & $23(48 \%)$ \\
\hline Age (years) & $48[21-86]$ & 60 [25-75] & $53[21-86]$ \\
\hline Weight (kg) & 79 [61-126] & 74 [54-96] & 77 [54-126] \\
\hline \multicolumn{4}{|l|}{ Tumor stage } \\
\hline$\| I_{A}$ & $1(4 \%)$ & 0 & $1(2 \%)$ \\
\hline\|\|$_{B}$ & $3(13 \%)$ & $2(8 \%)$ & $5(10 \%)$ \\
\hline IV & $19(83 \%)$ & $23(92 \%)$ & $42(88 \%)$ \\
\hline Brain metastases, yes & $3(13 \%)$ & $2(8 \%)$ & $5(10 \%)$ \\
\hline \multicolumn{4}{|c|}{ Previous lines of systemic therapy } \\
\hline 0 & $11(48 \%)$ & $17(68 \%)$ & $28(58 \%)$ \\
\hline 1 & $8(35 \%)$ & $6(24 \%)$ & $14(29 \%)$ \\
\hline$\geq 2$ & $4(17 \%)$ & $2(8 \%)$ & $6(13 \%)$ \\
\hline \multicolumn{4}{|l|}{ Crizotinib dose $^{a}$} \\
\hline $250 \mathrm{mg}$ BID & $16(70 \%)$ & $21(84 \%)$ & 37 (77\%) \\
\hline $200 \mathrm{mg}$ BID & $3(13 \%)$ & $2(8 \%)$ & $5(10 \%)$ \\
\hline 250 mg QD & $3(13 \%)$ & $2(8 \%)$ & $5(10 \%)$ \\
\hline 250 mg QAD & $1(4 \%)$ & 0 & $1(2 \%)$ \\
\hline \multicolumn{4}{|c|}{ WHO performance status } \\
\hline 0 & $16(70 \%)$ & $14(56 \%)$ & $30(63 \%)$ \\
\hline 1 & $7(30 \%)$ & $11(44 \%)$ & $18(38 \%)$ \\
\hline
\end{tabular}

Data are expressed as no. (\%) or median [range], as appropriate.

a Lowest dose per patient

$B I D=$ twice daily; $C_{\min }=$ minimum plasma concentration; $Q A D=$ every other day; $Q D=$ once daily; $W H O$

= World Health Organization 
Table 2 - Baseline characteristics of patients treated with alectinib $(n=52)$

\begin{tabular}{|c|c|c|c|}
\hline Patient characteristic & $\begin{array}{l}\text { Median } C_{\min } \\
<435 \mathrm{ng} / \mathrm{mL} \\
(\mathrm{n}=19)\end{array}$ & $\begin{array}{l}\text { Median } C_{\min } \\
\geq 435 \mathrm{ng} / \mathrm{mL} \\
(n=33)\end{array}$ & All patients \\
\hline Gender, female & $8(42 \%)$ & $20(61 \%)$ & $28(54 \%)$ \\
\hline Age (years) & 54 [21-70] & 60 [34-88] & 57 [21-88] \\
\hline Weight (kg) & $76[54-123]$ & 79 [49-117] & 78 [49-123] \\
\hline \multicolumn{4}{|l|}{ Tumor stage } \\
\hline$I I I_{A}$ & 0 & $1(3 \%)$ & $1(2 \%)$ \\
\hline$\| I I_{B}$ & 0 & $2(6 \%)$ & $2(4 \%)$ \\
\hline IV & $19(100 \%)$ & $30(91 \%)$ & $49(94 \%)$ \\
\hline Brain metastases, yes & $10(53 \%)$ & $10(30 \%)$ & $20(39 \%)$ \\
\hline \multicolumn{4}{|l|}{ Previous lines of systemic therapy } \\
\hline 0 & $3(16 \%)$ & $13(39 \%)$ & $16(31 \%)$ \\
\hline 1 & $8(42 \%)$ & $12(36 \%)$ & $20(39 \%)$ \\
\hline$\geq 2$ & $8(42 \%)$ & $8(24 \%)$ & $16(31 \%)$ \\
\hline Prior treatment with ALK-inhibitor(s), yes & $16(84 \%)$ & $19(58 \%)$ & $35(67 \%)$ \\
\hline \multicolumn{4}{|l|}{ Alectinib dose ${ }^{a}$} \\
\hline $600 \mathrm{mg}$ BID & $16(84 \%)$ & $15(45 \%)$ & $31(60 \%)$ \\
\hline 450 mg BID & $1(5 \%)$ & $8(24 \%)$ & $9(17 \%)$ \\
\hline $300 \mathrm{mg}$ BID & $2(11 \%)$ & $10(30 \%)$ & $12(23 \%)$ \\
\hline \multicolumn{4}{|l|}{ WHO performance status } \\
\hline 0 & $11(58 \%)$ & $6(18 \%)$ & $17(33 \%)$ \\
\hline 1 & $8(42 \%)$ & $21(64 \%)$ & $29(56 \%)$ \\
\hline$\geq 2$ & 0 & $4(12 \%)$ & $4(8 \%)$ \\
\hline missing & 0 & $2(6 \%)$ & $2(4 \%)$ \\
\hline
\end{tabular}

Data are expressed as no. (\%) or median [range], as appropriate

a Lowest dose per patient

$A L K=$ anaplastic lymphoma kinase; $B I D=$ twice daily; $C_{\min }=$ minimum plasma concentration; WHO = World Health Organization

In general, patients with a low exposure tended to be younger and had a more favourable World Health Organization (WHO) performance status (Table 1 and 2). For alectinib, patients with a low exposure were more often pre-treated with ALK-inhibitor(s) (i.e. crizotinib and/or ceritinib, Table 2).

Of the patients who were treated with crizotinib and alectinib sequentially and were included in both datasets $(n=17)$, seven patients had a low alectinib exposure, of whom five patients also had a low crizotinib exposure. Median follow-up was 43.6 months (range: 0.8-58.7 months) for crizotinib and 14.4 months (range: 2.2-24.6 months) for alectinib. Baseline characteristics of all patients treated with crizotinib (i.e. ALK-positive, ROS1-positive and CMET dysregulation) are provided in Supplementary Table 1. 


\section{Pharmacokinetic measurements}

Of the 100 consecutively included patients, a median of 3 samples per patient (range: 115) were available. In total, 376 PK samples were eligible for analysis ( 235 crizotinib and 141 alectinib). Figure 1 provides an overview of the median $C_{\min }$ per patient. Median crizotinib $C_{\min }$ per patient was $244 \mathrm{ng} / \mathrm{mL}$ (range: 103-688 ng/mL), with an interindividual and intra-individual variability of $45 \%$ and $20 \%$, respectively, at the standard dose of 250 mg BID. Median alectinib $C_{\text {min }}$ per patient was 517 ng/mL (range: 141-1944 ng/mL), with an interindividual and intra-individual variability of $57 \%$ and $27 \%$, respectively, at the standard dose of $600 \mathrm{mg}$ BID.

\section{Exposure-efficacy analysis}

Of the ALK-positive patients treated with crizotinib ( $n=48), 37$ patients (77\%) progressed, i.e. 20 patients (87\%) in the group with median $C_{\min }<235 \mathrm{ng} / \mathrm{mL}$ and 17 patients $(68 \%)$ in the group with median $C_{\min } \geq 235 \mathrm{ng} / \mathrm{mL}$. Intracranial progression occurred in 17 patients, i.e. 8 patients in the group with median $C_{\min }<235 \mathrm{ng} / \mathrm{mL}$ and 9 patients in the group with median $\mathrm{C}_{\min } \geq 235 \mathrm{ng} / \mathrm{mL}$. Median PFS in patients with crizotinib $\mathrm{C}_{\min }<235 \mathrm{ng} / \mathrm{mL}$ was 5.7 months (95\% confidence interval (Cl): 5.0-26.8 months), compared with 17.4 months (95\% $\mathrm{Cl}$ : 16.9-not estimable months) in patients with $C_{\min } \geq 235 \mathrm{ng} / \mathrm{mL}(\mathrm{p}=0.08$, log-rank test, Figure $2 A$ ). In multivariable analysis, $C_{\min }<235 \mathrm{ng} / \mathrm{mL}$ resulted in a hazard ratio (HR) of 1.79 (95\% Cl: 0.90-3.59, $\mathrm{p}=0.10$ ) when WHO performance status and the number of prior lines of treatment were taken into account (Table 3). A swimmer plot is shown in Supplementary Figure 2, which illustrates the treatment duration, dose reductions, and resistance mutations (when available) for each individual patient.

In the pooled analysis of all patients treated with crizotinib (i.e. ALK-positive, ROS1positive, and cMET dysregulation, $n=79$ ), median PFS in patients with crizotinib $C_{\min }<235$ $\mathrm{ng} / \mathrm{mL}$ was 5.3 months (95\% Cl: 4.9-15.9 months), compared with 11.8 months (95\% Cl: 8.7-18.1 months) in patients with $C_{\min }>235 \mathrm{ng} / \mathrm{mL}(\mathrm{p}=0.04$, log-rank test, Supplementary Figure 1). In multivariable analysis, $C_{\min }>235 \mathrm{ng} / \mathrm{mL}$ resulted in an $\mathrm{HR}$ of $2.15(95 \% \mathrm{Cl}$ : 1.21-3.84, $\mathrm{p}=0.009$ ) when mutation status, WHO performance status, and the number of prior lines of treatment were taken into account (Supplementary Table 2).

Of the patients treated with alectinib, 18 patients (35\%) progressed, i.e. 10 patients (53\%) in the group with median $\mathrm{C}_{\min }<435 \mathrm{ng} / \mathrm{mL}$ and 8 patients (24\%) in the group with median $C_{\min } \geq 435 \mathrm{ng} / \mathrm{mL}$. Intracranial progression occurred in four patients, i.e. three patients in the group with median $\mathrm{C}_{\min }<435 \mathrm{ng} / \mathrm{mL}$ and one patient in the group with median $\mathrm{C}_{\min } \geq$ $435 \mathrm{ng} / \mathrm{mL}$. Median PFS in patients with alectinib $C_{\min }<435 \mathrm{ng} / \mathrm{mL}$ was 12.6 months (95\% Cl: 9.2-not estimable months), compared with not estimable (95\% Cl: 19.8-not estimable months) in patients with $C_{\min } \geq 435 \mathrm{ng} / \mathrm{mL}$ ( $p=0.04$, log-rank test, Figure 2B). In multivariable analysis, $C_{\min } \geq 435 \mathrm{ng} / \mathrm{mL}$ resulted in an HR of 4.29 (95\% Cl: 1.33-13.90, $\mathrm{p}=0.015$ ) when WHO performance status and prior treatment with ALK-inhibitor(s) were 
taken into account (Table 4). A swimmer plot is shown in Supplementary Figure 3, which illustrates the treatment duration, dose reductions, and resistance mutations (when available) for each individual patient.
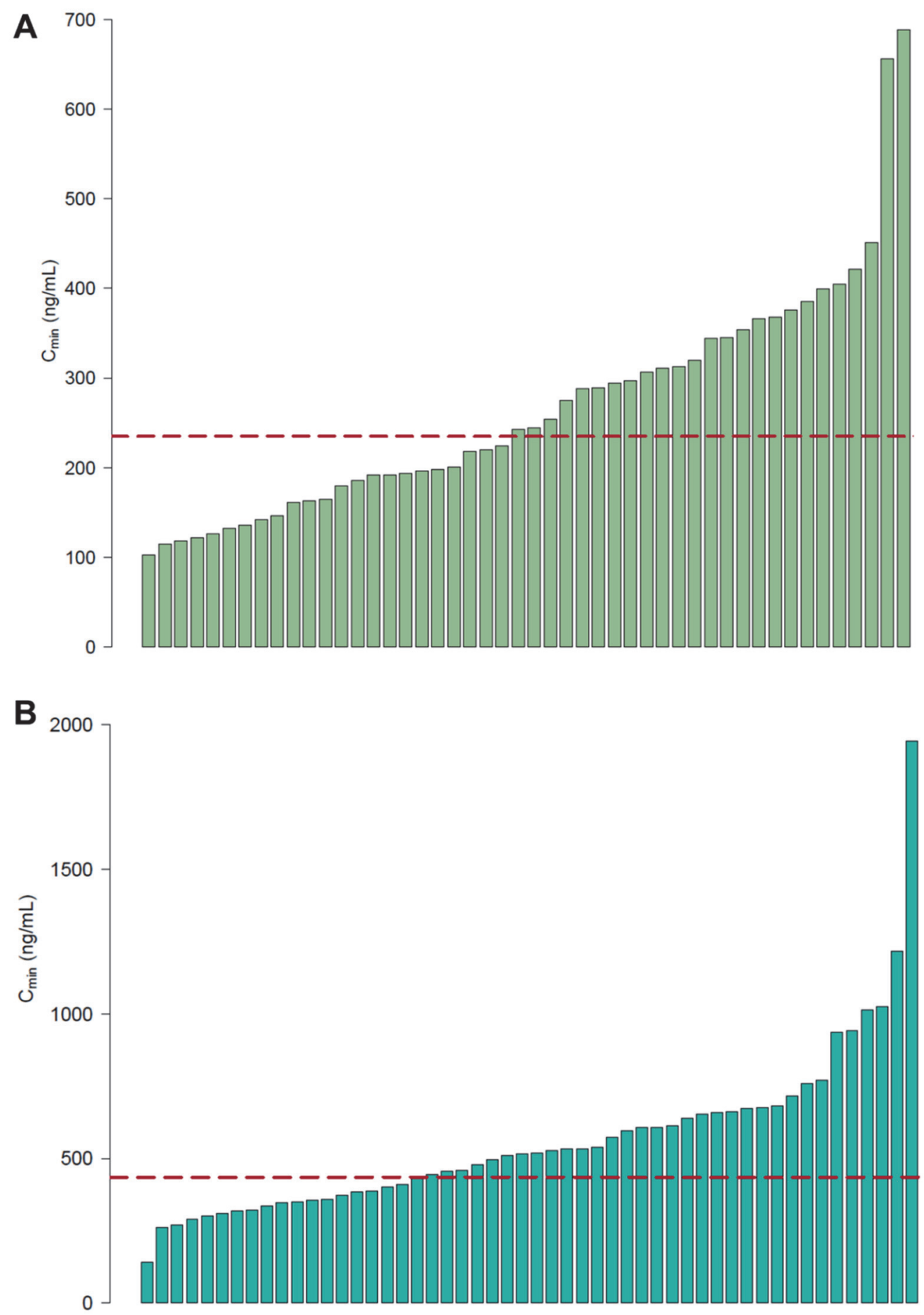

Figure 1 - Bar plots of median crizotinib and alectinib $C_{\min }$ per patient

A: Median crizotinib $C_{\min }$ per patient. Each bar represents one patient. The dotted line indicates the threshold of $235 \mathrm{ng} / \mathrm{mL}$. Twenty-three patients (48\%) have a pharmacokinetic exposure below this threshold. Interindividual and intra-individual variability were $45 \%$ and $20 \%$, respectively.

B: Median alectinib $C_{\min }$ per patient. Each bar represents one patient. The dotted line indicates the threshold of $435 \mathrm{ng} / \mathrm{mL}$. Nineteen patients (37\%) have a pharmacokinetic exposure below this threshold. Interindividual and intra-individual variability were $57 \%$ and $27 \%$, respectively.

$C_{\text {min }}=$ minimum plasma concentration 


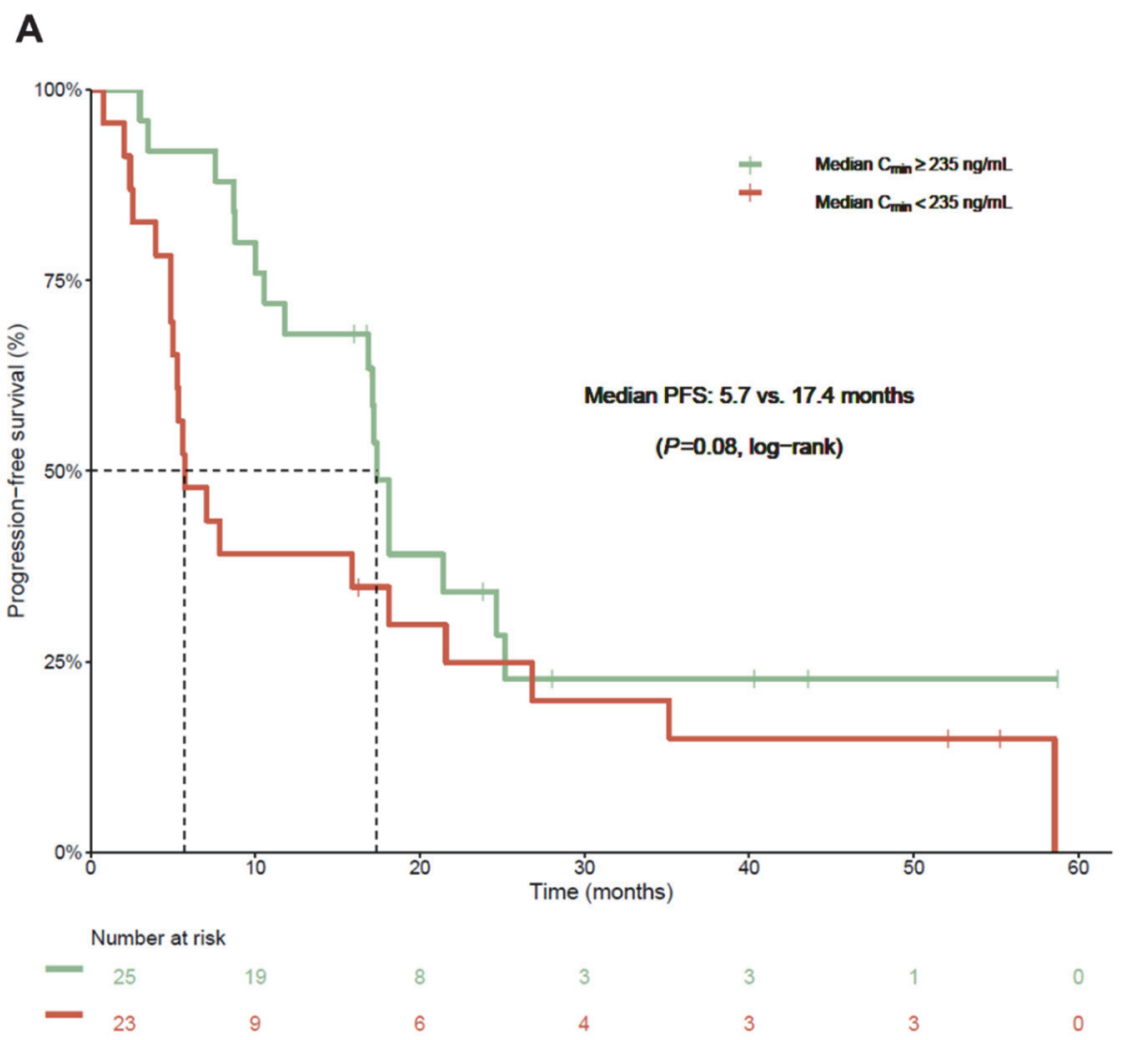

B

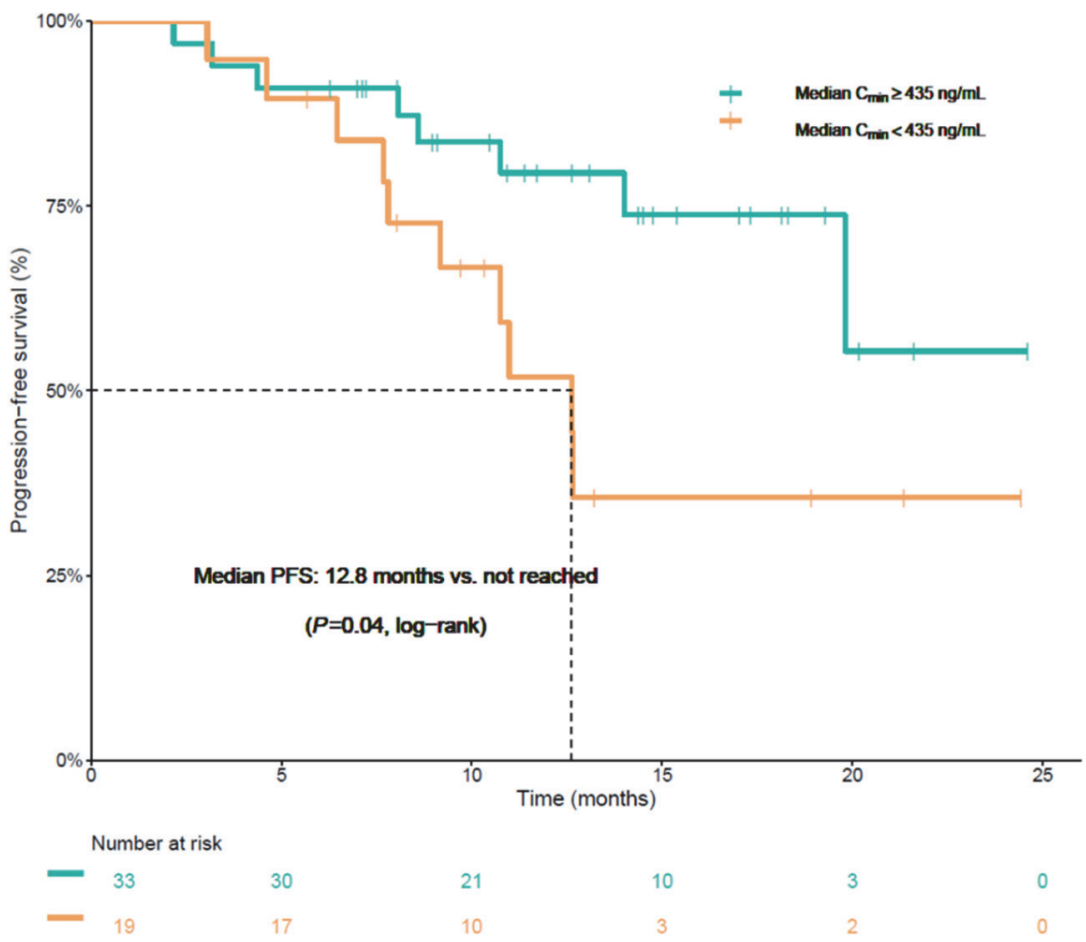

Figure 2 - Progression-free survival in patients treated with crizotinib and alectinib with an exposure above and below the proposed efficacy thresholds

A: Kaplan-Meier curve indicating the progression-free survival in patients treated with crizotinib with a median $C_{\min }$ above and below the threshold of $235 \mathrm{ng} / \mathrm{mL}$.

B: Kaplan-Meier curve indicating the progression-free survival in patients treated with alectinib with a median $C_{\min }$ above and below the threshold of $435 \mathrm{ng} / \mathrm{mL}$.

$C_{\text {min }}=$ minimum plasma concentration 
Table 3 - Univariable and multivariable Cox regression analysis for progression-free survival in patients treated with crizotinib

\begin{tabular}{lllllll}
\hline & \multicolumn{3}{c}{ Univariable } & \multicolumn{3}{c}{ Multivariable } \\
\hline Variable & HR & $\mathbf{9 5 \%} \mathbf{C l}$ & $\mathbf{p}$-value & HR & $\mathbf{9 5 \%} \mathbf{C l}$ & p-value \\
\hline $\mathbf{C}_{\min }<\mathbf{2 3 5} \mathbf{~ n g / \mathbf { m L }}$ & 1.76 & $0.92-3.39$ & 0.089 & 1.79 & $0.90-3.59$ & 0.100 \\
\hline Age (years) & 0.99 & $0.97-1.01$ & 0.295 & & & \\
\hline Gender, female & 0.65 & $0.34-1.27$ & 0.212 & & & 0.076 \\
\hline $\begin{array}{l}\text { WHO performance } \\
\text { status }\end{array}$ & 1.34 & $0.68-2.66$ & 0.398 & 1.97 & $0.93-4.15$ & \\
\hline $\begin{array}{l}\text { Number of prior lines of } \\
\text { treatment }\end{array}$ & 1.57 & $1.00-2.48$ & 0.052 & 1.61 & $1.01-2.58$ & 0.046 \\
\hline
\end{tabular}

$\mathrm{Cl}=$ confidence interval; $C_{\min }=$ minimum plasma concentration; $H R=$ hazard ratio; $W H O=$ World Health Organization

Table 4 - Univariable and multivariable Cox regression analysis for progression-free survival in patients treated with alectinib

\begin{tabular}{lllllll}
\hline & \multicolumn{3}{c}{ Univariable } & & \multicolumn{2}{c}{ Multivariable } \\
\hline Variable & HR & $\mathbf{9 5 \%} \mathbf{C l}$ & $\mathbf{p}$-value & HR & $\mathbf{9 5 \%} \mathbf{C l}$ & $\mathbf{p}$-value \\
\hline $\mathbf{C}_{\min }<\mathbf{4 3 5} \mathbf{n g} / \mathbf{m L}$ & 2.58 & $1.01-6.59$ & 0.047 & 4.29 & $1.33-13.90$ & 0.015 \\
\hline Age (years) & 0.99 & $0.95-1.02$ & 0.388 & & & \\
\hline Gender, female & 0.35 & $0.13-0.93$ & 0.035 & & & \\
\hline $\begin{array}{l}\text { WHO performance } \\
\text { status }\end{array}$ & 1.28 & $0.69-2.38$ & 0.428 & 2.35 & $1.07-5.16$ & 0.034 \\
\hline $\begin{array}{l}\text { Number of prior lines of } \\
\text { treatment }\end{array}$ & 1.65 & $1.05-2.61$ & 0.030 & & & \\
\hline $\begin{array}{l}\text { Prior treatment with } \\
\text { ALK-inhibitor(s) }\end{array}$ & 3.08 & $0.70-13.51$ & 0.136 & 2.81 & $0.57-13.94$ & 0.205 \\
\hline
\end{tabular}

Data were missing for two patients regarding WHO performance status.

$A L K=$ anaplastic lymphoma kinase; $C l=$ confidence interval; $C_{\min }=$ minimum plasma concentration; $H R$ = hazard ratio, $W H O=$ World Health Organization

\section{Exposure-toxicity analysis}

For crizotinib, 13 patients experienced clinically relevant toxicities (10 dose reductions, 7 dose interruptions, and 1 treatment discontinuation), including liver toxicity $(n=5)$, gastrointestinal toxicity $(n=2)$, pneumonitis $(n=1)$, neuropathy $(n=1)$, renal insufficiency $(n=1)$, neutropenia $(n=1)$, and fatigue $(n=1)$. In six of these patients, the toxicity event occurred before the first PK sample was collected. Median $\mathrm{C}_{\min }$ before the toxicity event was 338 ng/mL (range: $185-678$ ng/mL), compared with 264 ng/mL (range: 118-688 ng/mL) in patients without clinically relevant toxicities $(p=0.281)$. The two patients with the highest median $C_{\min }$ did not have clinically relevant toxicities with the currently used definition. However, the first patient (median $C_{\min } 656 \mathrm{ng} / \mathrm{mL}$ ) discontinued treatment due to cerebral progression, while at the same time she experienced symptoms possibly related to crizotinib (i.e. muscle weakness, ground glass opacities in the lungs and progression of kidney cysts). The second patient had a median $\mathrm{C}_{\min }$ of $688 \mathrm{ng} / \mathrm{mL}$ and died at the intensive 
care unit with an unknown cause of death, possibly due to cardiac arrhythmia, a recognised crizotinib toxicity.

For alectinib, 16 patients experienced clinically relevant toxicities (15 dose reductions, 7 dose interruptions), including edema $(n=6)$, fatigue $(n=4)$, myalgia $(n=3)$, gastrointestinal toxicity $(n=3)$, bradycardia $(n=2)$, liver toxicity $(n=2)$, skin rash $(n=1)$, anemia $(n=1)$, and renal insufficiency $(n=1)$. In addition, six patients started at a lower dose, due to severe toxicity during prior treatment with crizotinib $(n=4)$, elevated liver enzymes $(n=1)$, and miscommunication between patient and physician $(n=1)$. Median $C_{\min }$ in patients with and without clinically relevant toxicities was $539 \mathrm{ng} / \mathrm{mL}$ and $431 \mathrm{ng} / \mathrm{mL}$, respectively ( $p=0.205$ ).

\section{DISCUSSION}

In this observational study we investigated whether pharmacokinetic exposure to ALKinhibitors crizotinib and alectinib is related to treatment efficacy and toxicity. Patients with a median alectinib $C_{\min } \geq 435 \mathrm{ng} / \mathrm{mL}$ had a significantly longer median PFS compared with patients with an exposure below this threshold (12.6 months vs. not reached yet, Figure 2B, Table 4)). For crizotinib, median PFS was also numerically longer in patients with a median $C_{\min } \geq 235 \mathrm{ng} / \mathrm{mL}$ (5.7 vs. 17.4 months), which is a clinically relevant difference, although this difference was not statistically significant (Figure 2A, Table 3). In the pooled analysis, which also included ROS1-positive and CMET-dysregulated patients, statistical significance was reached (Supplementary Figure 1, Supplementary Table 2). Exposure to both crizotinib and alectinib was not significantly related to clinically relevant toxicities.

Interindividual variability in pharmacokinetic exposure was found to be considerable (i.e. $45-57 \%$ ), which is in line with previous literature. ${ }^{6,7}$ As a consequence, $48 \%$ of the patients treated with crizotinib and 37\% of the patients treated with alectinib had an exposure below the efficacy threshold, and were found at risk of decreased treatment efficacy. This implies that individualized dosing is indicated in this subgroup of patients with a low exposure to improve treatment outcomes, for which we provide practical recommendations in Figure 3.

Apart from the high interindividual variability, intra-individual variability in alectinib exposure was also found to be large (i.e. $27 \%$ ). This could be caused by the substantial food effect of alectinib, as its exposure increases more than fourfold when administered with a high-fat meal compared with fasting conditions. ${ }^{16}$ According to the label, alectinib is administered concomitantly with food, but the content and volume of these meals could vary substantially within patients over time.

Many patients needed a dose reduction due to toxicity or started treatment at a lower dose (i.e. $23 \%$ for crizotinib and $40 \%$ for alectinib, Table 1 and 2). It is notable that, especially for alectinib, many of these patients still had an adequate exposure. Of the patients with a low exposure to crizotinib and alectinib, 30\% and 16\%, respectively, 
received a prior dose reduction due to toxicity. This means that $70 \%$ and $84 \%$ of the low exposed patients, respectively, in the absence of toxicity, would potentially benefit from dose escalation.

Notably, patients with a low exposure to alectinib were more often pre-treated with other ALK-inhibitors (84\% compared with $58 \%$ of patients with adequate exposure). Similar underlying factors (i.e. increased clearance or decreased absorption) could have caused a low exposure to both alectinib and the previous ALK-inhibitor(s). These patients may, therefore, have failed treatment with the previous ALK-inhibitor(s) earlier due to their low exposure and needed subsequent treatment with alectinib sooner. In addition, an inherently lower treatment adherence in these patients could have played a role.

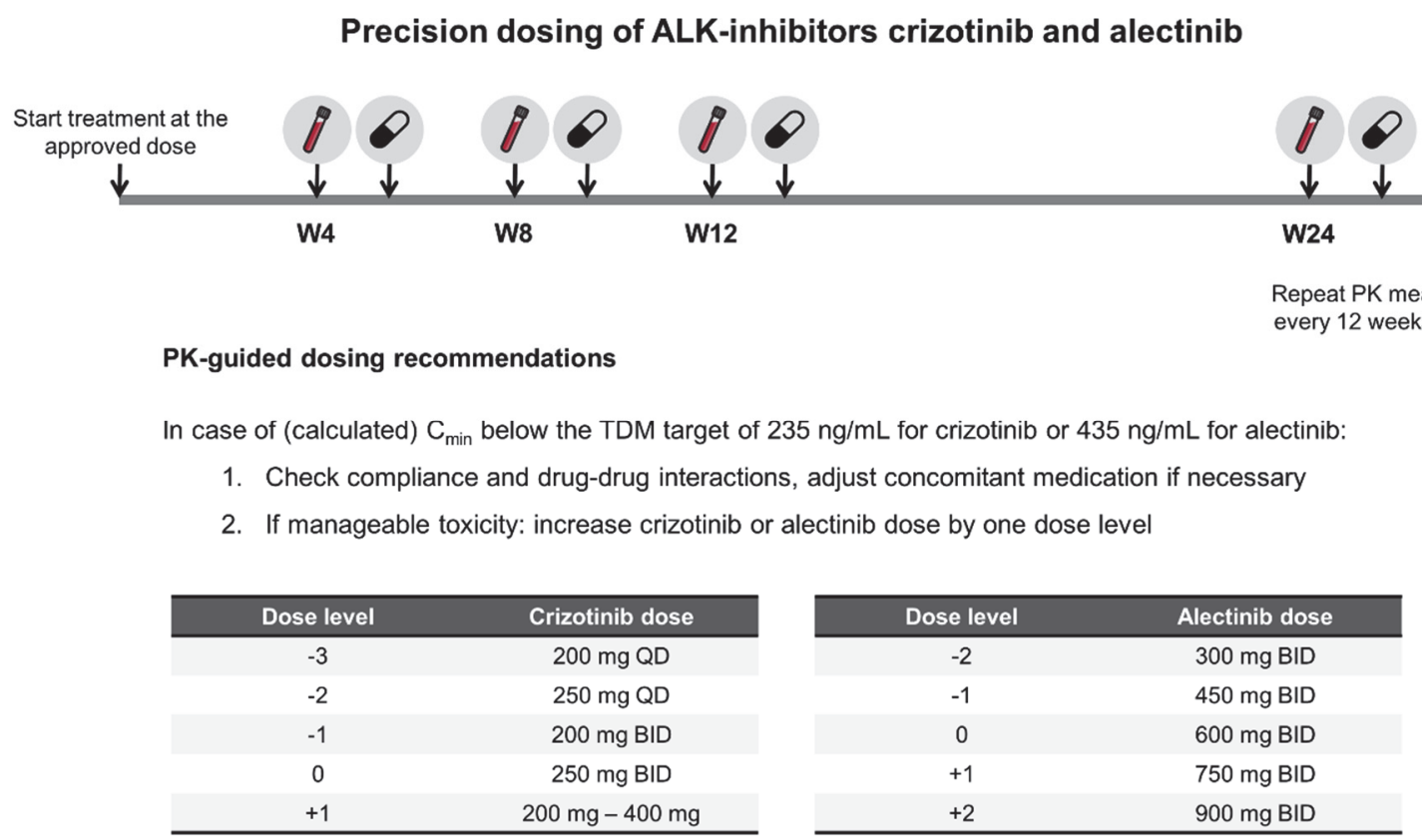

Figure 3 - Practical recommendations for precision dosing of crizotinib and alectinib

Patients start treatment at the approved dose of $250 \mathrm{mg}$ BID for crizotinib and $600 \mathrm{mg}$ BID for alectinib. PK samples will be collected 4,8 and 12 weeks after start of treatment and every 12 weeks thereafter. In case of (calculated) $C_{\min }$ below the TDM target of $235 \mathrm{ng} / \mathrm{mL}$ for crizotinib or 435 $\mathrm{ng} / \mathrm{mL}$ for alectinib and manageable toxicity, the dose will be increased by one dose level (after checking treatment adherence and drug-drug interactions). Maximum dose levels are based on data from phase I dose finding studies.

$B I D=$ twice daily,$C_{\min }=$ minimum plasma concentration, $P K=$ pharmacokinetic(ally), $Q D=$ once daily, $T D M=$ therapeutic drug monitoring, $W=$ week

While alectinib is indicated only for the treatment of ALK-positive NSCLC, crizotinib is also approved for ROS1-positive NSCLC and used off-label in the treatment of patients with CMET dysregulation (i.e. amplification or exon 14 skipping). Due to the similarity of ALK and ROS1 kinase domains, crizotinib has similar half maximal inhibitory concentrations values of 40-60 nM and $60 \mathrm{nM}$ against ALK and ROS1, respectively, while the half maximal inhibitory concentration against CMET was notably lower (i.e. $8 \mathrm{nM}$ ). ${ }^{17} \mathrm{It}$ could, therefore, be hypothesized that the efficacy threshold of $C_{\min } \geq 235 \mathrm{ng} / \mathrm{mL}$, that was established in 
ALK-positive patients, will also hold true for ROS1-positive patients, while a lower threshold might be sufficient for patients with cMET dysregulation. Since these subgroups have a different prognosis and the efficacy threshold might be different, exposure-efficacy analyses should preferably be performed separately for each subgroup. However, to further increase our sample size, we did perform a pooled analysis, in which we accounted for mutation status, resulting in a statistically significant exposure-response relationship.

In a previously performed exposure-response analysis in patients treated with alectinib $(n=207)$, no association has been identified between median $C_{\min }$ of alectinib and its active metabolite M4 and overall survival. ${ }^{18}$ Although overall survival is regarded as the gold standard metric of treatment outcome, an exposure-efficacy relationship can easily be diluted by the effects of successive treatment lines. ${ }^{4}$ In this study, a potential relationship with PFS has not been evaluated. Furthermore, no threshold was tested and adjustments to measured concentrations were performed to account for analytical bias (due to crossvalidation issues). ${ }^{18}$ More recent analyses across the phase III studies did demonstrate a relationship between exposure and PFS. They identified an optimal pharmacokinetic threshold of $1040 \mathrm{nM}$ for the sum of alectinib and M4, corresponding to an alectinib $C_{\text {min }}$ of approximately $370 \mathrm{ng} / \mathrm{mL} .{ }^{19}$ Since only limited data on this analysis is currently available in the literature, we did not use this cut-off value in our analyses.

An important strength of the current study is that data were collected from real-life patients, instead of from highly selected patients in a clinical trial. This is the first time that an exposure-response relationship is described outside the context of a clinical trial for ALK-inhibitors, which is relevant, since treatment in a real-life setting may differ considerably from treatment in a clinical trial setting (i.e. no drug accountability, more complex patients who would not be eligible for a trial). In addition, multiple samples over time were available for most patients, providing an adequate reflection of pharmacokinetic exposure during treatment.

On the other hand, limitations of this study include that the applied method to estimate $C_{\text {min }}$ assumes an equal alectinib clearance in all patients, not taking into account interindividual differences in the elimination half-life. In addition, part of the samples (up to $59 \%$, Supplementary Figure 4) were collected during the absorption or distribution phase (i.e. before the time to maximum concentration), resulting in an underestimation of $C_{\min }$. However, due to the long $t_{1 / 2}$ (i.e. 42 hours for crizotinib and 32 hours for alectinib) in respect to the dosing interval (i.e. 12 hours), differences between $C_{\min }$ and $C_{\max }$ are small with a peak-to-trough ratio of approximately 1.3. ${ }^{20,21}$ Therefore, the deviations from the actual trough levels can be considered acceptable. These imprecisions could have been circumvented by drawing actual trough levels, but this is less convenient for patients as the collection of PK samples is usually combined with regular visits to the outpatient clinic. Another approach would be to use population PK models to estimate $C_{\min }$, which would allow for taking into account the interpatient variability in PK parameters. However, 
disadvantages of this approach are its increased complexity and the fact that Bayesian estimation based on limited sampling will result in shrinkage towards the typical value. ${ }^{22}$ Furthermore, another limitation is that selection bias could have played a role, as some patients may have discontinued treatment before the first sample was drawn. These patients may have had early progression due to low exposure. Finally, $C_{\min }$ might not be the most appropriate PK parameter to assess exposure-toxicity relationships. Although trough levels are critical to ensure maximal target engagement during the complete dosing interval at the tumor level, other PK parameters may better reflect the potential relationship between exposure and side effects (i.e. AUC or $C_{\max }$ ).

It is known that the emergence of resistance mutations causes treatment failure. ${ }^{23} \mathrm{An}$ interesting concept that needs to be further elucidated is whether the prevalence of these resistance mutations is equally high in patients with a low pharmacokinetic exposure compared with patients with an adequate pharmacokinetic exposure. In Supplementary Figure $\mathbf{2}$ and $\mathbf{3}$ we report the identified resistance mutations in our patient cohorts. However, as resistance mutation analysis was only performed in a small subset of patients $(n=17)$, we have insufficient data to answer this question.

Future steps will be to evaluate the feasibility, tolerability, and efficacy of individualized dosing of crizotinib and alectinib, which will be studied in an ongoing prospective study on therapeutic drug monitoring (i.e. adjusting the dose based on measured drug levels) of oral anticancer drugs. ${ }^{24}$ Although a randomized controlled trial comparing a therapeutic drug monitoring strategy to a flat dosing strategy could be considered the gold standard, this also assumes an equipoise between treatment arms. Therefore, it could be questioned if performing a randomized controlled trial is ethical where a clear exposure-response relationship exists.

In addition, it would be interesting to investigate the role of the active metabolite of alectinib (i.e. M4). This metabolite has a similar potency as alectinib itself, but a much lower abundance $\left( \pm 30 \%\right.$ of the parent). ${ }^{7}$ Concentrations of M4 follow the alectinib concentrations, although data are very limited. Concomitant administration of alectinib with cytochrome P450 3A4 isozyme inhibitors or inducers results in inverse changes in alectinib and M4 concentrations, without affecting the total exposure to a clinically relevant degree. ${ }^{25}$ This should be kept in mind when therapeutic drug monitoring of alectinib is applied without measuring M4. It should be noted that all patients in the current study were carefully monitored for drug-drug interactions in clinical practice and, therefore, strong or moderate cytochrome P450 3A4 isozyme inhibitors or inducers were not used in this cohort. A combined threshold of the sum concentration of alectinib plus M4 may further improve precision dosing of alectinib. But given the above considerations, we think the addition of M4 will not relevantly change the finding of this study. 
In conclusion, here we demonstrated that exposure to crizotinib and alectinib is related to efficacy in a real-life patient cohort, providing a strong rationale for therapeutic drug monitoring. Individualized dosing based on therapeutic drug monitoring may improve treatment outcomes for the subgroup of patients with a $\mathrm{C}_{\min }$ below the efficacy thresholds of $235 \mathrm{ng} / \mathrm{mL}$ for crizotinib and $435 \mathrm{ng} / \mathrm{mL}$ for alectinib.

\section{ACKNOWLEDGEMENTS}

We thank everyone who contributed to the logistics of collecting and measuring the crizotinib and alectinib concentrations, in particular Remy Verheijen, Laura MolenaarKuijsten and René Boosman. This work was presented in part at the 2019 ESMO Annual Meeting, Barcelona (Annals of Oncology, Volume 30, Issue Supplement_5, October 2019, mdz260.008, https://doi.org/10.1093/annonc/mdz260.008). 


\section{REFERENCES}

1. Soda $M$, Isobe $K$, Inoue A, Maemondo M, Oizumi S, Fujita $Y$ et al. A prospective PCR-based screening for the EML4-ALK oncogene in non-small cell lung cancer. Clin Cancer Res 2012; 18: 5682-5689.

2. Horn L, Pao W. EML4-ALK: honing in on a new target in non-small-cell lung cancer. J Clin Oncol 2009; 27: 4232-4236.

3. Zhao F, Xu M, Lei H, Zhou Z, Wang L, Li P et al. Clinicopathological characteristics of patients with non-small-cell lung cancer who harbor EML4-ALK fusion gene: a meta-analysis. PLoS One 2015; 10: 1-13.

4. Solomon BJ, Kim D-W, Wu YL, Nakagawa K, Mekhail T, Felip E et al. Final overall survival analysis from a study comparing first-line crizotinib versus chemotherapy in ALK-mutationpositive non-small-cell lung cancer. J Clin Oncol 2018; 36: 2251-2258.

5. Peters S, Camidge DR, Shaw AT, Gadgeel S, Ahn JS, Kim DW et al. Alectinib versus crizotinib in untreated ALK-positive non-small-cell lung cancer. N Eng/J Med 2017; 377: 829-838.

6. Food and Drug Administration. Center for Drug Evaluation and Research. Crizotinib Clinical Pharmacology and Biopharmaceutics Review. 2011. Available from: http://www.accessdata.fda.gov/drugsatfda_docs/nda/2011/2025700rig1s000ClinPharmR.p df.

7. Food and Drug Administration. Center for Drug Evaluation and Research. Alectinib Clinical Pharmacology and Biopharmaceutics Review. 2016. Available from: http://www.accessdata.fda.gov/drugsatfda_docs/nda/2015/208434Orig1s000ClinPharmR.p df.

8. Mitchell AP, Harrison MR, Walker MS, George DJ, Abernethy AP, Hirsch BR. Clinical trial participants with metastatic renal cell carcinoma differ from patients treated in real-world practice. J Oncol Pract 2015; 11: 491-497.

9. Herbrink M, de Vries N, Rosing H, Huitema ADR, Nuijen B, Schellens JHM et al. Development and validation of a liquid chromatography - tandem mass spectrometry analytical method for the therapeutic drug monitoring of eight novel anticancer drugs. Biomed Chromatogr 2018; 38: 1-9.

10. Janssen JM, de Vries N, Venekamp N, Rosing H, Huitema ADR, Beijnen JH. Development and validation of a liquid chromatography-tandem mass spectrometry assay for nine oral anticancer drugs in human plasma.J Pharm Biomed Anal 2019; 174: 561-566.

11. Wang Y, Chia Y, Nedelman J, Schran H, Mahon F, Molimard M. A therapeutic drug monitoring algorithm for refining the imatinib trough level obtained at different sampling times. Ther Drug Monit 2009; 31: 579-584.

12. Food and Drug Administration. Center for Drug Evaluation and Research. Imatinib Clinical Pharmacology and Biopharmaceutics Review. 2001. Available from: https://www.accessdata.fda.gov/drugsatfda_docs/nda/2001/21335_Gleevec_biopharmr_P1.pdf.

13. Verheijen RB, $\mathrm{Yu} H$, Schellens JHM, Beijnen JH, Steeghs N, Huitema ADR. Practical recommendations for therapeutic drug monitoring of kinase inhibitors in oncology. Clin Pharmacol Ther 2017; 102: 765-776. 
14. R Core Development Team. A language and environment for statistical computing. R Found Statistical Computing, Vienna. 2016. Available from: https://www.r-project.org/.

15. Schemper $\mathrm{M}$, Smith TL. A note on quantifying follow-up in studies of failure time. Control Clin Trials 1996; 17: 343-346.

16. Morcos PN, Guerini E, Parrott N, Dall G, Blotner S, Bogman K et al. Effect of food and esomeprazole on the pharmacokinetics of alectinib, a highly selective ALK inhibitor, in healthy subjects. Clin Pharmacol Drug Dev 2017; 6: 388-397.

17. Lin JJ, Shaw AT. Recent advances in targeting ROS1 in lung cancer. J Thorac Oncol 2017; 12: 1611-1625.

18. Morcos PN, Nueesch E, Jaminion F, Guerini E, Hsu JC, Bordogna W et al. Exposure-response analysis of alectinib in crizotinib-resistant ALK-positive non-small cell lung cancer. Cancer Chemother Pharmacol 2018; 82: 129-138.

19. Smart K, Hsu JC, Jaminion F, Guerini E, Shaw AT, Zhou C et al. Alectinib exposure-response (ER) in ALK-inhibitor naive ALK-positive NSCLC patients: pooled analysis across phase III studies. J Clin Oncol 2019; 37: e20575.

20. Hirota T, Muraki S, leiri I. Clinical pharmacokinetics of anaplastic lymphoma kinase inhibitors in non-small-cell lung cancer. Clin Pharmacokinet 2019; 58: 403-420.

21. Gadgeel SM, Gandhi L, Riely GJ, Chiappori AA, West HL, Azada MC et al. Safety and activity of alectinib against systemic disease and brain metastases in patients with crizotinib-resistant ALK-rearranged non-small-cell lung cancer (AF-002JG): results from the dose-finding portion of a phase 1/2 study. Lancet Oncol 2014; 15: 1119-1128.

22. Janssen JM, Dorlo TPC, Beijnen JH, Huitema ADR. Evaluation of extrapolation methods to predict trough concentrations to guide therapeutic drug monitoring of oral anticancer drugs. Ther Drug Monit 2020; 42: 532-539.

23. Gainor JF, Dardaei L, Yoda S, Friboulet L, Leshchiner I, Katayama R et al. Molecular mechanisms of resistance to first- and second-generation ALK inhibitors in ALK-rearranged lung cancer. Cancer Discov 2016; 6: 1118-1133.

24. Groenland SL, van Eerden RAG, Verheijen RB, Koolen SLW, Moes DJAR, Desar IME et al. Therapeutic drug monitoring of oral anticancer drugs: the DPOG-TDM protocol for a prospective study. Ther Drug Monit 2019; 41: 561-567.

25. Morcos PN, Cleary Y, Guerini E, Dall G, Bogman K, De Petris L et al. Clinical drug-drug interactions through cytochrome P450 3A (CYP3A) for the selective ALK inhibitor alectinib. Clin Pharmacol Drug Dev 2017; 6: 280-291. 


\section{SUPPLEMENTARY DATA}

Supplementary Table 1 - Baseline characteristics of all patients treated with crizotinib (i.e. ALK-positive, ROS1-positive and CMET-dysregulated patients)

\begin{tabular}{|c|c|c|c|}
\hline Patient characteristic & $\begin{array}{l}\text { Median } C_{\min } \\
<235 \mathrm{ng} / \mathrm{mL} \\
(\mathrm{n}=34)\end{array}$ & $\begin{array}{l}\text { Median } C_{\min } \\
\geq 235 \mathrm{ng} / \mathrm{mL} \\
(\mathrm{n}=45)\end{array}$ & $\begin{array}{l}\text { All patients } \\
(n=79)\end{array}$ \\
\hline Gender, female & $11(32 \%)$ & $24(53 \%)$ & $35(44 \%)$ \\
\hline Age (years) & $54 \pm 15$ & $60 \pm 12$ & $57 \pm 14$ \\
\hline \multicolumn{4}{|l|}{ Mutation } \\
\hline ALK & $23(68 \%)$ & $25(56 \%)$ & $48(61 \%)$ \\
\hline ROS1 & $4(12 \%)$ & $10(22 \%)$ & $14(18 \%)$ \\
\hline cMET amplification & $7(21 \%)$ & $7(16 \%)$ & $14(18 \%)$ \\
\hline cMET exon 14 skipping & 0 & $3(7 \%)$ & $3(4 \%)$ \\
\hline \multicolumn{4}{|l|}{ Tumor stage } \\
\hline$\| \mathrm{I}_{\mathrm{A}}$ & $1(3 \%)$ & 0 & $1(1 \%)$ \\
\hline$\|I\|_{B}$ & $3(9 \%)$ & $2(4 \%)$ & $5(6 \%)$ \\
\hline IV & $30(88 \%)$ & $43(96 \%)$ & $73(92 \%)$ \\
\hline Brain metastases, yes & $7(21 \%)$ & $6(13 \%)$ & $13(17 \%)$ \\
\hline \multicolumn{4}{|c|}{ Previous lines of systemic therapy } \\
\hline 0 & $14(41 \%)$ & $23(51 \%)$ & $37(47 \%)$ \\
\hline 1 & $12(35 \%)$ & $19(42 \%)$ & $31(39 \%)$ \\
\hline$\geq 2$ & $7(21 \%)$ & $3(7 \%)$ & $10(13 \%)$ \\
\hline \multicolumn{4}{|l|}{ WHO performance status } \\
\hline 0 & 20 (59\%) & $19(42 \%)$ & 39 (49\%) \\
\hline 1 & $13(38 \%)$ & $22(49 \%)$ & 35 (44\%) \\
\hline 2 & 0 & $3(7 \%)$ & $3(4 \%)$ \\
\hline
\end{tabular}

$\overline{A L K}=$ anaplastic lymphoma kinase; $c M E T=$ mesenchymal epithelial transition growth factor; $C_{\min }=$ minimum plasma concentration; ROS1 = c-ros oncogene $1 ; W H O=$ World Health Organization 
Supplementary Table 2 - Univariable and multivariable Cox regression analysis for progression-free survival in all patients treated with crizotinib (i.e. ALK-positive, ROS1positive and CMET-dysregulated patients)

\begin{tabular}{|c|c|c|c|c|c|c|}
\hline & \multicolumn{3}{|c|}{ Univariable } & \multicolumn{3}{|c|}{ Multivariable } \\
\hline Variable & HR & $95 \% \mathrm{Cl}$ & p-value & HR & $95 \% \mathrm{Cl}$ & p-value \\
\hline$C_{\min }<235 \mathrm{ng} / \mathrm{mL}$ & 1.69 & $1.03-2.78$ & 0.039 & 2.15 & $1.21-3.84$ & 0.009 \\
\hline \multicolumn{7}{|l|}{ Mutation status ${ }^{a}$} \\
\hline ROS1 & 1.61 & $0.79-3.29$ & 0.188 & 1.11 & $0.45-2.78$ & 0.818 \\
\hline cMET amplification & 2.80 & $1.46-5.37$ & 0.002 & 2.45 & $1.22-4.91$ & 0.011 \\
\hline $\begin{array}{l}\text { CMET exon } 14 \\
\text { skipping }\end{array}$ & 2.93 & $0.87-9.93$ & 0.083 & 2.99 & $0.83-10.68$ & 0.093 \\
\hline Age (years) & 1.00 & $0.98-1.01$ & 0.588 & & & \\
\hline Gender, female & 0.79 & $0.48-1.31$ & 0.365 & & & \\
\hline WHO performance status & 1.71 & $1.07-2.74$ & 0.026 & 1.88 & $1.08-3.27$ & 0.025 \\
\hline $\begin{array}{l}\text { Number of prior lines of } \\
\text { treatment }\end{array}$ & 1.29 & $0.99-1.68$ & 0.063 & 1.11 & $0.82-1.51$ & 0.502 \\
\hline
\end{tabular}

a hazard ratio compared to ALK-positive patients

$A L K=$ anaplastic lymphoma kinase; $\mathrm{Cl}=$ confidence interval; $C M E T=$ mesenchymal epithelial transition growth factor; $C_{\min }=$ minimum plasma concentration; $H R=$ hazard ratio; $R O S 1=c$-ros oncogene $1 ; W H O$ $=$ World Health Organization 


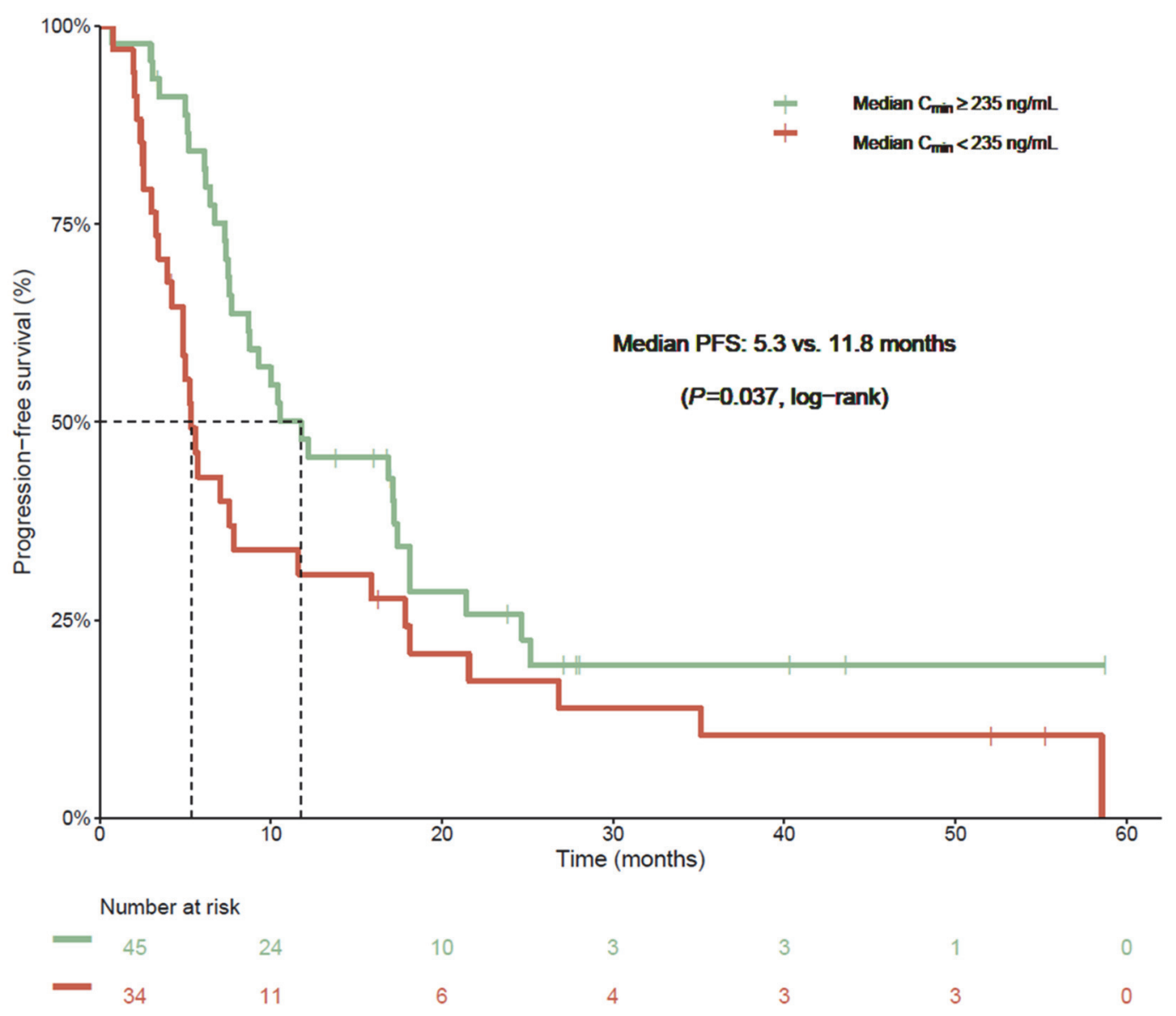

Supplementary Figure 1 - Kaplan-Meier curve of progression-free survival in all patients treated with crizotinib (i.e. ALK-positive, ROS1-positive and cMET-dysregulated patients)

Kaplan-Meier curve indicating the progression-free survival in all patients treated with crizotinib (i.e. ALK-positive, ROS1-positive and cMET-dysregulated patients) with a median $\mathrm{C}_{\min }$ above and below the threshold of $235 \mathrm{ng} / \mathrm{mL}$.

$A L K=$ anaplastic lymphoma kinase; $C M E T=$ mesenchymal epithelial transition growth factor; $R O S 1=c-$ ros oncogene 1 


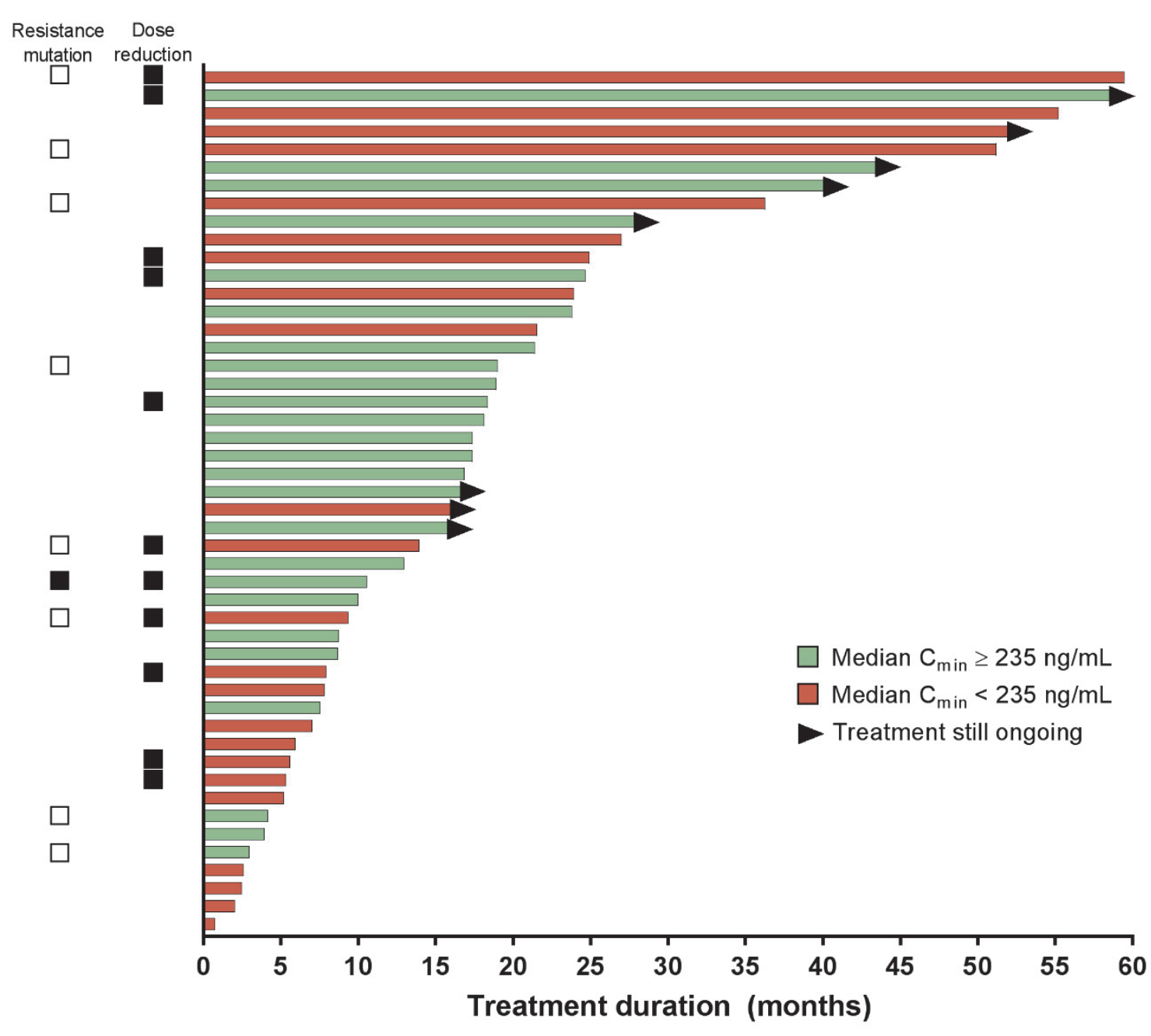

Each bar represents one patient

\section{Supplementary Figure $\mathbf{2}$ - Swimmer plot of patients treated with crizotinib}

Each bar represents one patient. White boxes indicate patients in whom the presence of resistance mutations was evaluated at disease progression, but in whom none were identified. The black box indicates the one patient in whom a resistance mutation was identified (i.e. ALK G1202R). $A L K=$ anaplastic lymphoma kinase $; C_{\min }=$ minimum plasma concentration 


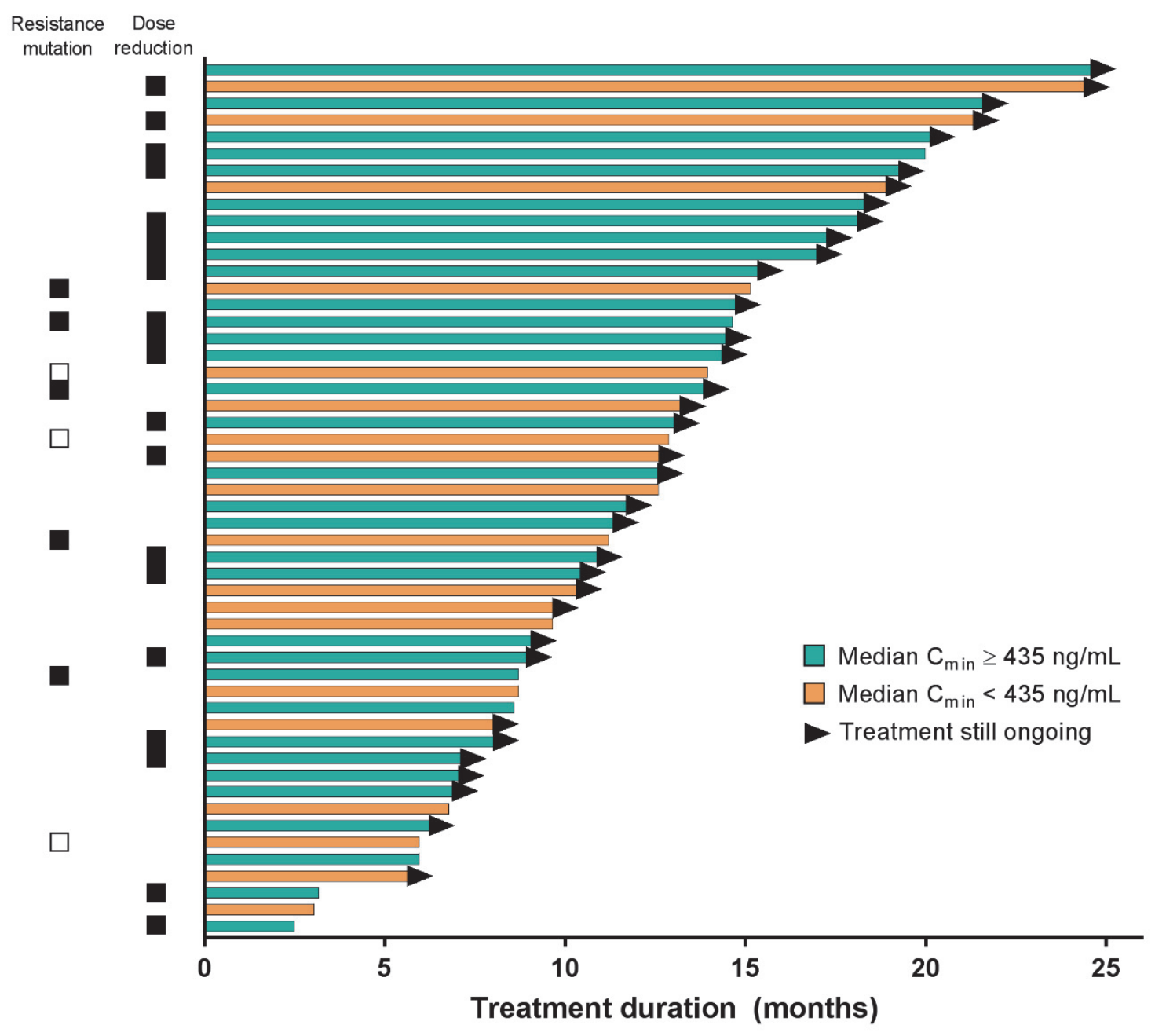

Each bar represents one patient

\section{Supplementary Figure 3 - Swimmer plot of patients treated with alectinib}

Each bar represents one patient. White boxes indicate patients in whom the presence of resistance mutations was evaluated at disease progression, but in whom none were identified. Black boxes indicate the patients in whom a resistance mutation was identified (i.e. ALK G1202R ( $n=3)$ and ALK $11171 \mathrm{~N}(\mathrm{n}=2))$.

$A L K=$ anaplastic lymphoma kinase; $C_{\min }=$ minimum plasma concentration 

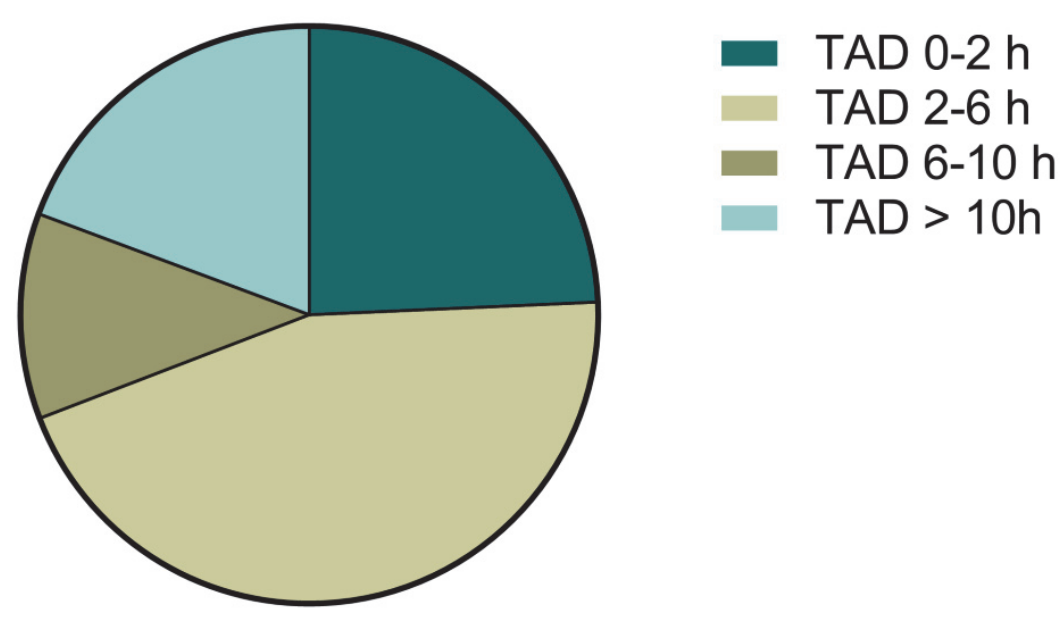

\section{1 crizotinib samples}

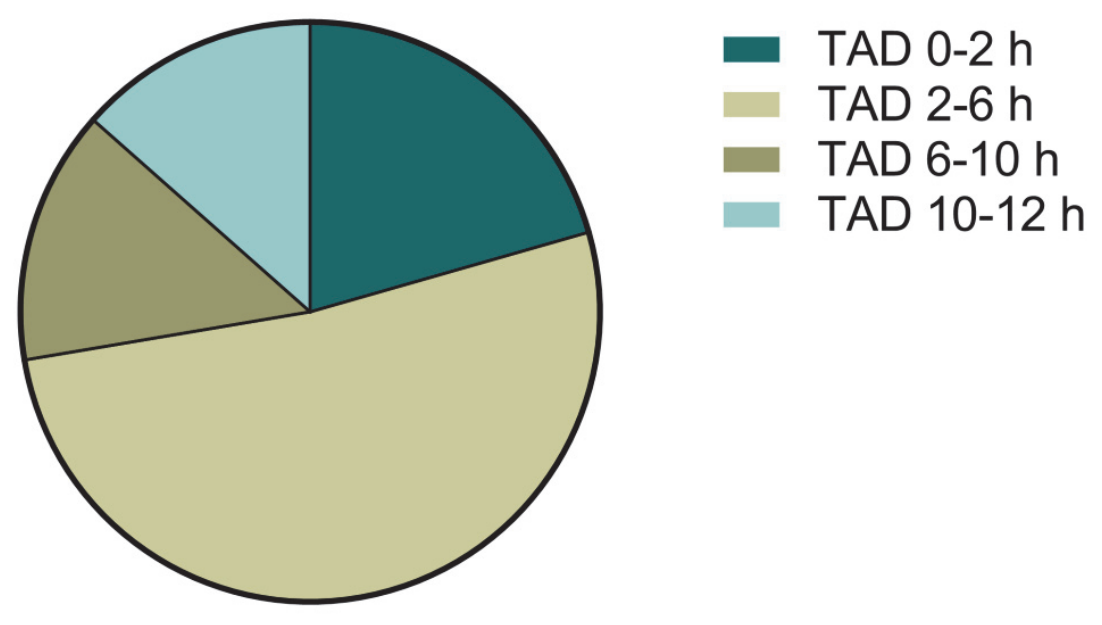

141 alectinib samples

Supplementary Figure 4 - Pie charts of the distribution of time after dose for crizotinib and alectinib samples

$T A D=$ time after dose 


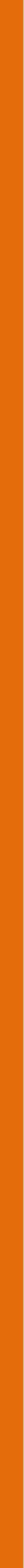





\section{PART III}

Precision dosing in clinical practice 


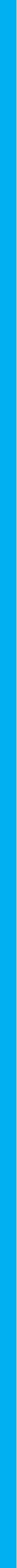




\section{ABSTRACT}

\section{Background}

Oral anticancer drugs show a high interpatient variability in pharmacokinetics (PK), leading to large differences in drug exposure. For many of these drugs, exposure has been linked to efficacy and toxicity. Despite this knowledge, these drugs are still administered in a one-size-fits-all approach. Consequently, individual patients have a high probability to be either underdosed, which can lead to decreased antitumor efficacy, or overdosed, which could potentially result in increased toxicity. Therapeutic drug monitoring (TDM), personalized dosing based on measured drug levels, could be used to circumvent underdosing and overdosing and thereby optimize treatment outcomes.

\section{Methods}

In this prospective clinical study (www.trialregister.nl; NL6695), the feasibility, tolerability, and efficacy of TDM of oral anticancer drugs will be evaluated. In total, at least 600 patients will be included for (at least) 23 different compounds. Patients starting regular treatment with one of these compounds at the approved standard dose can be included. PK sampling will be performed at 4, 8, and 12 weeks after start of treatment and every 12 weeks thereafter. Drug concentrations will be measured, and trough concentrations $\left(C_{\min }\right)$ will be calculated. In case where $C_{\min }$ falls below the predefined target and acceptable toxicity, a PK-guided intervention will be recommended. This could include emphasizing compliance, adapting concomitant medication (due to drug-drug interactions), instructing to take the drug concomitantly with food, splitting intake moments, or recommending a dose increase.

\section{Discussion}

Despite a strong rationale for the use of TDM for oral anticancer drugs, this is currently not yet widely adopted in routine patient care. This prospective study will be a valuable contribution to demonstrate the additional value of dose optimization on treatment outcome for these drugs. 


\section{BACKGROUND}

Although in the past century intravenously administered chemotherapy has always formed the backbone of cancer therapy, this paradigm has shifted in the past two decades toward personalized treatment in which oral anticancer drugs are indispensable. Despite the high interpatient variability in pharmacokinetic (PK) exposure and the fact that for most of these oral anticancer drugs, an exposure-response relationship has been identified, these drugs are still administered at fixed doses. As a consequence, individual patients have a high probability to be either underdosed ( $>30 \%$ of patients) or overdosed ( $>15 \%$ of patients), leading to decreased antitumor efficacy and increased toxicity, respectively. ${ }^{1-6}$ Therapeutic drug monitoring (TDM), personalized dosing based on measured drug levels, can be used to address these problems and thereby optimize treatment.

Practical guidelines for TDM of kinase inhibitors and oral antihormonal drugs have been developed and published previously. ${ }^{4,5}$ Also, feasibility studies have been performed for several anticancer drugs, and they showed TDM to be feasible and safe..$^{1,2,6-8}$

Despite the strong rationale for TDM, it has not yet been implemented as the standard of care in clinical practice. Reasons for this include reimbursement and regulatory issues for higher than approved doses of these expensive drugs and reimbursement of drug level measurement. In addition, clinicians might be reluctant to increase the dose in fear of toxicity, although several studies have shown TDM to be feasible and safe., ${ }^{1,2,6-8}$ Furthermore, indisputable evidence on the efficacy of TDM, demonstrated in prospective studies, is lacking. Although randomized controlled trials (RCTs) are considered the gold standard in evidence-based medicine, it would be challenging to perform a RCT on TDM of oral anticancer drugs. This is mainly because a high number of patients with mostly rare cancers would be needed and it would be challenging to secure funding for this. These difficulties are illustrated by the premature termination of a randomized trial of TDM in imatinib patients. ${ }^{9}$ Also, it could be argued that not performing dose increments in part of the patients is unethical when clear exposure-response relationships exist. ${ }^{10}$

Therefore, it is necessary to obtain prospective clinical data on the feasibility, tolerability, and efficacy of TDM of oral anticancer drugs. In this study, we aim to implement TDM for these drugs in multiple large medical centers across the Netherlands, assembled in the Dutch Pharmacology Oncology Group (DPOG, www.dpog.nl) and to build a prospective registry to structurally collect data on patients' clinical outcome and the effectiveness of the interventions. 


\section{METHODS}

The DPOG-TDM study is a multicenter investigator-initiated prospective clinical study. Patients with a regular indication for selected oral anticancer agents start treatment at the standard approved dose according to the label, which includes regular monitoring on drug-drug interactions, contra-indications, and other treatment-specific parameters. Then, drug levels will be measured at 4, 8, and 12 weeks after start of treatment and every 12 weeks thereafter, except for compounds with intermittent dosing schedules or a long elimination half-life $\left(\mathrm{t}_{1 / 2}\right)$. An overview of the PK sampling schedule per compound can be found in Table 1. For each of these agents, detailed drug-specific TDM and dosing guidelines have been formulated based on the currently available evidence and best practice (see Supplemental Data File, http://links.Iww.com/TDM/A341). According to the (calculated) trough levels $\left(C_{\min }\right)$ of the drug and the reported toxicities, treatment recommendations will be provided to the treating physician. This could include PK-guided interventions such as emphasizing compliance, adapting concomitant medication (due to drug-drug interactions), instructing the patients to take the drug concomitantly with food, splitting intake moments, or recommending a dose increase.

In total, at least 600 patients will be included for 23 different oral anticancer drugs, with a possibility to extend with additional agents (and patients) when additional funding is secured.

Figure 1 presents a schematic overview of the study design. Table 1 summarizes the PK sampling schedules, TDM targets, and dose levels per drug.

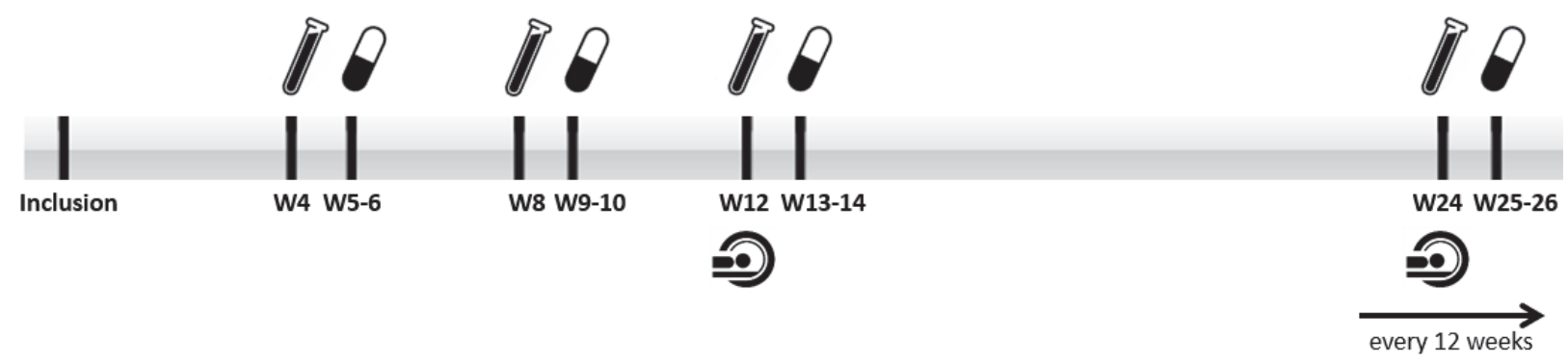

PK samples will be drawn 4, 8 and 12 weeks after start of treatment and every 12 weeks thereafter, except for compounds with a long half-life or an intermittent dosing schedule (for more details see Table 1)

TDM recommendations will be provided to the treating physician, these could include PK-guided interventions such as emphasizing compliance, adaptations in concomitant medication (due to drug-drug interactions), instructions to take the drug concomitant with food, splitting intake moments or the recommendation to increase the dose

20 Tumor assessments will be performed according to standard of care

Figure 1 - Study schedule

$P K=$ pharmacokinetic; TDM = therapeutic drug monitoring; $W=$ week 


\section{Objectives}

The primary objective of this study is to halve the proportion of patients with a drug exposure below the TDM target after two potential PK-guided interventions, which for most compounds will be after 12 weeks. Table $\mathbf{2}$ provides the historically presented fraction of patients with an exposure below the TDM target, which will be used as comparison.

The secondary objectives of this study are to determine the tolerability and feasibility of PK-guided dosing, to determine the objective response rate (according to the Response Evaluation Criteria In Solid Tumors (RECIST) version 1.1), to determine the time to tumor progression and progression-free survival, to determine the proportion of patients with drug exposure below the TDM target after one potential PK-guided intervention, and to have a physician adherence of $>90 \%$ in following the provided treatment recommendations. Objective response rate will be defined as the proportion of patients with confirmed complete response or confirmed partial response according to RECIST version 1.1. Time to tumor progression will be defined as the time from start of treatment to the first documentation of objective tumor progression. Progression-free survival will be defined as the time from the start of treatment to the first documentation of objective tumor progression or to death due to any cause, whichever occurs first.

\section{Inclusion criteria}

Patients are eligible for this study if they are aged 18 years or older, have a diagnosis of cancer, an indication to start treatment with one of the oral anticancer drugs included in the study protocol, have a World Health Organization (WHO) performance status of 0, 1, or 2, have a life expectancy of at least 3 months, allowing adequate follow-up of toxicity and antitumor efficacy, and are willing to provide written informed consent.

\section{Exclusion criteria}

Patients are excluded if they start treatment at a reduced dose, are known with alcoholism, drug addiction, and/or a psychiatric or physiological condition, which, in the opinion of the investigator, would impair treatment compliance, have any other disease, neurological or metabolic dysfunction, physical examination finding or laboratory finding giving reasonable suspicion of a disease or condition that contra-indicates the use of the drug or puts the patient at high risk of treatment-related complications, or are legally incapable.

\section{TDM targets}

The TDM targets used in this study are based on previously published practical guidelines on TDM of kinase inhibitors and oral antihormonal drugs and are shown in Table 1. ${ }^{4,5}$ For most drugs (16 of 23), this TDM target is based on exposure-efficacy 
Table 1 - Summary of TDM targets, PK parameters, and defined dose levels per oral anticancer drug

\begin{tabular}{|c|c|c|c|c|c|c|c|c|}
\hline Drug & $\begin{array}{l}\text { Indica- } \\
\text { tion }\end{array}$ & $\begin{array}{l}\text { TDM } \\
\text { target }^{a} \\
(\mathrm{ng} / \mathrm{mL})\end{array}$ & $\begin{array}{l}t_{1 / 2} \\
\text { (h) }\end{array}$ & $\begin{array}{l}t_{\max } \\
\text { (h) }\end{array}$ & $\begin{array}{l}\text { Starting } \\
\text { dose }\end{array}$ & $\begin{array}{l}\text { Stepwise } \\
\text { increases } \\
\text { in daily } \\
\text { dose per } \\
\text { dose level } \\
\text { (mg) }\end{array}$ & $\begin{array}{l}\text { Maximum } \\
\text { dose }\end{array}$ & Remarks \\
\hline Abiraterone & PC & 8.4 & 12 & 2 & $\begin{array}{l}1000 \mathrm{mg} \\
\text { QD }\end{array}$ & 250 & $\begin{array}{l}1500 \mathrm{mg} \\
\text { QD }\end{array}$ & $\begin{array}{l}\text { First step is intake } \\
\text { concomitant with } \\
\text { light meal or } \\
\text { snack }\end{array}$ \\
\hline Alectinib & NSCLC & 435 & 32 & 4 & $\begin{array}{l}600 \mathrm{mg} \\
\text { BID }\end{array}$ & 300 & $900 \mathrm{mg}$ BID & \\
\hline Axitinib & RCC & 5 & 4 & 3 & $5 \mathrm{mg}$ BID & $4-6$ & $10 \mathrm{mg}$ BID & \\
\hline Bosutinib & CML & 147 & 34 & 6 & $\begin{array}{l}500 \mathrm{mg} \\
\text { QD }\end{array}$ & 100 & $600 \mathrm{mg}$ QD & \\
\hline Cobimetinib $^{b}$ & Mel & 127 & 44 & 2 & $60 \mathrm{mg}$ QD & 20 & $100 \mathrm{mg}$ QD & \\
\hline Crizotinib & NSCLC & 235 & 42 & 5 & $\begin{array}{l}250 \mathrm{mg} \\
\text { BID }\end{array}$ & 100 & $\begin{array}{l}200 \mathrm{mg}- \\
400 \mathrm{mg}\end{array}$ & \\
\hline Dasatinib & CML & 2.61 & 6 & 1 & $\begin{array}{l}100 \mathrm{mg} \\
\text { QD }\end{array}$ & 40 & $180 \mathrm{mg}$ QD & \\
\hline Enzalutamide $^{c}$ & PC & 5000 & 139 & 1 & $\begin{array}{l}160 \mathrm{mg} \\
\text { QD }\end{array}$ & 40 & $240 \mathrm{mg}$ QD & \\
\hline Erlotinib & NSCLC & 500 & 36 & 4 & $\begin{array}{l}150 \mathrm{mg} \\
\text { QD }\end{array}$ & 50 & 300 mg QD & \\
\hline Everolimus & $\begin{array}{l}\text { RCC, } \\
\text { NET, BC }\end{array}$ & 10 & 38 & 1 & $10 \mathrm{mg}$ QD & 2.5 & 15 mg QD & \\
\hline Gefitinib & NSCLC & 200 & 41 & 5 & $\begin{array}{l}250 \mathrm{mg} \\
\text { QD }\end{array}$ & 250 & 750 mg QD & \\
\hline Imatinib & $\begin{array}{l}\text { GIST, } \\
\text { CML }\end{array}$ & $1100^{d}$ & 19 & 3 & $\begin{array}{l}400 \mathrm{mg} \\
\text { QD }\end{array}$ & 200 & $400 \mathrm{mg}$ BID & $\begin{array}{l}\text { In case of very } \\
\text { low } P K\left(C_{\min }<550\right. \\
\mathrm{ng} / \mathrm{mL}) \text {, an } \\
\text { increase of two } \\
\text { dose levels will be } \\
\text { recommended }\end{array}$ \\
\hline Nilotinib & CML & 469 & 17 & 3 & $\begin{array}{l}300 \mathrm{mg} \\
\text { BID }\end{array}$ & 200 & $600 \mathrm{mg}$ BID & \\
\hline Olaparib & OC & 1290 & 12 & 2 & $\begin{array}{l}400 \mathrm{mg} \\
\text { BID }\end{array}$ & 200 & $600 \mathrm{mg}$ BID & \\
\hline Palbociclib & BC & 61 & 27 & 4 & $\begin{array}{l}125 \mathrm{mg} \\
\text { QD }\end{array}$ & 25 & $200 \mathrm{mg}$ BID & \\
\hline Pazopanib & $\begin{array}{l}\text { RCC, } \\
\text { STS }\end{array}$ & 20500 & 31 & 4 & $\begin{array}{l}800 \mathrm{mg} \\
\text { QD }\end{array}$ & 200 & $\begin{array}{l}1000 \mathrm{mg} \\
\text { BID }\end{array}$ & $\begin{array}{l}\text { First step is to } \\
\text { split intake } \\
\text { moments into } \\
400 \mathrm{mg} \mathrm{BID,} \\
\text { second step is } \\
\text { intake } \\
\text { concomitant with } \\
\text { food }\end{array}$ \\
\hline Regorafenib $^{b}$ & $\begin{array}{l}\text { CRC, } \\
\text { GIST }\end{array}$ & 1400 & 28 & 4 & $\begin{array}{l}160 \mathrm{mg} \\
\text { QD }\end{array}$ & 40 & 200 mg QD & \\
\hline Sorafenib & $\mathrm{HCC}, \mathrm{TC}$ & 3750 & 26 & 8 & $\begin{array}{l}400 \mathrm{mg} \\
\text { BID }\end{array}$ & 200 & $800 \mathrm{mg}$ BID & \\
\hline
\end{tabular}




\begin{tabular}{|c|c|c|c|c|c|c|c|c|}
\hline Sunitinib $^{\mathbf{e}}$ & $\begin{array}{l}\text { GIST, } \\
\text { NET, } \\
\text { RCC }\end{array}$ & $50^{f}$ & $\begin{array}{l}50 / \\
95^{\mathrm{g}}\end{array}$ & 5 & $50 \mathrm{mg}$ QD & 12.5 & $75 \mathrm{mg}$ QD & \\
\hline Tamoxifen $^{\text {h }}$ & $B C$ & 5.97 & 336 & 2 & $20 \mathrm{mg}$ QD & 10 & $60 \mathrm{mg}$ QD & $\begin{array}{l}\text { In case of very } \\
\text { low PK }\left(C_{\text {steady-state }}\right. \\
<3 \mathrm{ng} / \mathrm{mL}) \text {, an } \\
\text { increase of two } \\
\text { dose levels will be } \\
\text { recommended }\end{array}$ \\
\hline Trametinibi $^{i}$ & $\mathrm{Mel}$ & 10.6 & 96 & 2 & $2 \mathrm{mg}$ QD & 0.5 & $3 \mathrm{mg}$ QD & \\
\hline Vemurafenib & $\mathrm{Mel}$ & 42000 & 34 & 5 & $\begin{array}{l}960 \mathrm{mg} \\
\text { BID }\end{array}$ & 480 & $\begin{array}{l}1440 \mathrm{mg} \\
\text { BID }\end{array}$ & \\
\hline Vismodegib $^{h}$ & $\mathrm{BCC}$ & 11.4 & 96 & 4 & $\begin{array}{l}150 \mathrm{mg} \\
\text { QD }\end{array}$ & 150 & 450 mg QD & \\
\hline
\end{tabular}

a TDM target concentrations are all $C_{\min }$, except for tamoxifen, for which the TDM target refers to the steadystate concentration of its active metabolite endoxifen

${ }^{\mathrm{b}}$ Because these drugs have an intermittent dosing schedule, PK samples will be drawn 3, 7, and 11 weeks after the start of treatment

c Because enzalutamide has a long half-life ( \pm 6 days), PK samples will be drawn 4, 10, and 16 weeks after the start of treatment

${ }^{\mathrm{d}}$ For CML patients, the TDM target is $\mathrm{C}_{\min } \geq 1000 \mathrm{ng} / \mathrm{mL}$

e For patients receiving sunitinib in an intermittent dosing schedule, PK samples will be drawn 4, 10, and 16 weeks after the start of treatment

f TDM target for intermittent dosing schedule is $C_{\min } \geq 50 \mathrm{ng} / \mathrm{mL}$ (sum of concentrations of both sunitinib and its active metabolite $\mathrm{N}$-desethylsunitinib), whereas for continuous dosing schedule, TDM target is $C_{\min } \geq 37.5$ $\mathrm{ng} / \mathrm{mL}$

$\mathrm{g}_{1 / 2}$ is different for sunitinib (50 hours) and its active metabolite $\mathrm{N}$-desethylsunitinib (95 hours)

${ }^{h}$ Because (active metabolites of) these drugs have a very long half-life, PK samples will be drawn each 12 weeks

' For dabrafenib, drug concentrations will only be measured, no dose adaptations will be recommended because this might not be the ideal drug for TDM

$B C=$ breast cancer; $B C C=$ basal cell carcinoma; $B I D=$ twice daily; $C_{\min }=$ minimum plasma concentration $/$ trough concentration; $C_{\text {steady-state }}=$ steady-state concentration; $C M L=$ chronic myelogenous leukemia; $C R C=$ colorectal cancer; GIST = gastrointestinal stromal tumor; HCC = hepatocellular carcinoma; Mel = melanoma; NET = neuroendocrine tumor; NSCLC = non-small-cell lung cancer; $O C=$ ovarian cancer; $P K=$ pharmacokinetics; $Q D=$ once daily; $P C=$ prostate cancer; $R C C=$ renal cell carcinoma; $S T S=$ soft tissue sarcoma; $T C=$ thyroid cancer; $T D M=$ therapeutic drug monitoring; $t_{\max }=$ time to maximum concentration; $t_{1 / 2}=$ elimination half-life

analyses. If these analyses were not available (yet), the mean or median exposure of the drug was taken as a reference. For the compounds with TDM targets based on exposureefficacy analyses, the PK targets amounted to $81-85 \%$ of the average population exposure. ${ }^{4,5}$ Therefore, targeting the mean or median concentration will generally lead to an efficacious exposure. In the meantime, thorough exposure-efficacy analyses will be awaited, which can provide a definitive target for TDM.

\section{Dose levels}

Levels for dose adjustments have been defined for each drug, indicating the maximum dose of the drug and the steps with which the dose should be increased in case of low exposure or decreased in case of toxicity. The highest dose level is based on the maximum tolerated dose found in the phase I study. If the maximum tolerated dose was not 
reached, the highest dose tested in the phase I study was taken as the maximum dose. In case of saturated absorption, concomitant intake with food (abiraterone and pazopanib) or splitting intake moments (pazopanib) will be recommended, based on findings from previous studies. ${ }^{32,33}$ In Table 1, the maximum dose levels and stepwise increases are reported for each drug. All dose levels per drug are described in the Supplemental Data File (http://links.Iww.com/TDM/A341).

Table 2 - Historical data of the percentage of patients below TDM target per oral anticancer drug

\begin{tabular}{|c|c|c|}
\hline Drug & $\begin{array}{l}\text { Patients below TDM target } \\
\text { at standard dose (\%) }\end{array}$ & Reference \\
\hline Abiraterone & 35 & 11 \\
\hline Alectinib & 33 & 12 \\
\hline Axitinib & 38 & 13 \\
\hline Bosutinib & 50 & 14 \\
\hline Cobimetinib & 50 & 15 \\
\hline Crizotinib & 25 & 16 \\
\hline Dasatinib & 50 & 17 \\
\hline Enzalutamide & 2 & 18 \\
\hline Erlotinib & 11 & 3 \\
\hline Everolimus & 37 & 19 \\
\hline Gefitinib & 26 & 20 \\
\hline Imatinib & 73 & 3 \\
\hline Nilotinib & 25 & 21 \\
\hline Olaparib & 50 & 22 \\
\hline Palbociclib & 50 & 23 \\
\hline Pazopanib & $16-20$ & 24,25 \\
\hline Regorafenib & 50 & 26 \\
\hline Sorafenib & 50 & 27 \\
\hline Sunitinib & 49 & 3 \\
\hline Tamoxifen & 20 & 28 \\
\hline Trametinib $^{a}$ & 27 & 29 \\
\hline Vemurafenib & 52 & 30 \\
\hline Vismodegib & 50 & 31 \\
\hline
\end{tabular}

a Data reported only for trametinib, as for dabrafenib, no dose adjustments will be recommended because little evidence for an exposure-response relationship for dabrafenib is available.

$T D M=$ therapeutic drug monitoring

\section{Pharmacokinetic measurements}

Concentrations of the drug will be measured using validated liquid chromatographytandem mass spectrometry assays. ${ }^{34-39}$ Quality of measurements will be secured by interlaboratory comparison. Patients will be instructed to let the blood sample be drawn after the time to maximum concentration $\left(t_{\max }\right)$ of the drug has been reached. Each time 
a PK sample is drawn, the patient will be asked the date and time of the last drug intake, and this will be recorded, as well as the time of blood sampling, to calculate the time after dose. Trough concentrations will then be calculated based on the time after dose and the $t_{1 / 2}$ of the drug using the following formula:

$$
C_{\text {min }}=C_{\text {measured }} * 0.5 \frac{\text { dosing interval-TAD }}{t_{1 / 2}}
$$

where $C_{\text {min }}$ is the minimum drug concentration, $C_{\text {measured }}$ is the measured drug concentration, TAD is the time after dose, and $t_{1 / 2}$ is the average elimination half-life of the drug. ${ }^{40}$

Table 1 shows the PK sampling schedule per drug and the corresponding $t_{\max }$ and $t_{1 / 2}$ values, which will be used to calculate the trough levels.

\section{PK-guided interventions}

If the estimated trough concentration is below the predefined TDM target and the patient does not show any treatment-related $\geq$ grade 3 toxicity, a PK-guided intervention will be recommended to the treating physician within 1-2 weeks. This could include emphasizing compliance, adapting concomitant medication (due to drug-drug interactions), instructing to take the drug concomitantly with food, splitting intake moments, or recommending a dose increase. If patients show any $\geq$ grade 3 toxicity, the dose will be interrupted until the toxicity is $\leq$ grade 1 . If the toxicity was treatment-related, the dose will be reduced with one dose level.

In case of concentrations below the TDM target, compliance will be checked directly with the patient. If compliance seems to be the cause of low PK exposure, no dose increments will be performed. Instead, compliance will be emphasized, and a new PK sample will be drawn after steady-state concentrations have been reached again. In this way, compliance will be assessed before making dose increases.

\section{Tolerability and efficacy assessments}

Toxicity will be evaluated during routine visits to the outpatient clinics. Tumor assessments according to RECIST version 1.1 will be performed at least every 12 weeks as part of standard care.

\section{Statistics: sample size}

The primary objective of this study is to halve the proportion of patients with a PK exposure below the predefined TDM target after two PK-guided interventions. If we consider the percentages reported in the literature as historical controls (Table 2), then using an exact binomial test with a nominal 0.05 two-sided significance level will provide the power as indicated in Table 3 assuming different levels of the null and alternative 
hypothesis and various sample sizes. Obviously, if a higher proportion of patients have a low PK exposure, fewer patients are needed to provide a reasonable power. Sample size calculations were performed using the power.binom.test function of the pwr package in R. $^{41}$

Table 3 - Sample size calculation showing power at different levels of null and alternative hypothesis and three examples of sample size

\begin{tabular}{|c|c|c|c|c|}
\hline \multirow{2}{*}{\multicolumn{2}{|c|}{$\begin{array}{c}\text { Proportion of patients with a } \\
\text { drug exposure below TDM } \\
\text { target }\end{array}$}} & \multicolumn{3}{|c|}{ Number of patients } \\
\hline & & 30 & 60 & 90 \\
\hline Null & Alternative & \multicolumn{3}{|c|}{ Power $(\%, 1-\beta)$} \\
\hline 0.10 & 0.05 & 1 & 19 & 33 \\
\hline 0.20 & 0.10 & 18 & 43 & 71 \\
\hline 0.30 & 0.15 & 32 & 71 & 92 \\
\hline 0.40 & 0.20 & 60 & 92 & 98 \\
\hline 0.50 & 0.25 & 80 & 97 & \\
\hline 0.60 & 0.30 & 91 & & \\
\hline
\end{tabular}

TDM = therapeutic drug monitoring

Regarding the secondary outcome of evaluating the tolerability and feasibility of TDM, generally $\pm 25-30 \%$ of the total patient group will be eligible for dose escalation. To assess the feasibility of PK-guided interventions in at least 8 patients, about 3-4 times as many patients need to be included. Therefore, the aim is to include at least 30 patients per compound. For abiraterone, imatinib, pazopanib, sunitinib, and trametinib, patient inclusion will be expanded to be able to evaluate the influence of TDM on efficacy as well.

\section{Statistics: analysis}

The full analysis set will include all patients who received at least one dose of the oral anticancer drug. Patients will only be considered evaluable for the primary endpoint if they have completed the first three PK measurements. An exact binomial test will be performed for each drug. In some situations of drug and target combinations, it may prove difficult to obtain a sufficient number of patients for an acceptable level of power. Therefore, an additional meta-analytic approach will be applied to test the "proof-ofprinciple" of TDM. For each drug, the standardized change in percentage of patients with a concentration below the predefined target at the third PK measurement after the start of treatment will be calculated. Secondary endpoints will be described using descriptive statistics.

\section{Logistic and administrative arrangements}

The DPOG-TDM study was assessed by the accredited Medical Ethics Committee of the Netherlands Cancer Institute - Antoni van Leeuwenhoek Hospital (NKI-AVL) on May 3, 2017, and it was decided that the study did not fall under the Dutch Medical Research Involving Human Subjects Act because no additional procedures are required for the 
participants. The institutional review board authorized the study on August 7, 2017. Patients do need to give written informed consent because data will be collected and shared. The study protocol follows the principles of the Declaration of Helsinki and the code of conduct of the Dutch FEDERA guidelines.

The NKI-AVL is the coordinating center. Other participating centers are the Erasmus Medical Center (EMC), Radboud University Medical Center (Radboud UMC), Leiden University Medical Center (LUMC), and University Medical Center Groningen (UMCG). Additional participating centers of the study are currently being recruited.

Data on baseline characteristics, measured blood concentrations, TDM recommendations, dose adjustments, toxicity, efficacy, and survival will be collected in the electronical case report form. Members of the study team will have access to the final dataset.

\section{DISCUSSION}

Currently, all patients treated with oral anticancer drugs receive a standard fixed dose (i.e. all patients receive exactly the same dose, independent of their weight or body surface area), although it is well known that these drugs show a large interpatient variability, and for many of them, exposure has been linked to efficacy and adverse events, providing a strong rationale for TDM. Also, other strategies such as body surface area-based dosing do not lead to an improvement in PK exposure. ${ }^{42}$ This study aims to demonstrate the added value of TDM in collaborating hospitals.

In the development of the study design, several choices had to be made. Ideally, one would choose to perform a RCT. However, this would require an even larger sample size. Also, it could be considered unethical to fail to increase the dose in case of measured low exposure when a clear relationship between exposure and efficacy exists. Therefore, we decided to perform this prospective intervention trial.

Furthermore, we decided to only recommend dose increments in case of low PK exposure, whereas it could be argued that dose reductions in case of high PK exposure might be beneficial as well, as this could be associated with less toxicity and lower costs. However, in oncology, cautions are warranted regarding dose reductions based on PK exposure because disease progression is an irreversible event. Of course, dose reductions would be made in case of toxicity, as this is regular routine patient care, which is also included in the labels.

In addition, we chose to calculate $C_{\min }$ using the above-mentioned formula based on time after dose and the average elimination half-life of the drug, assuming a one-compartment model and that distribution has largely been completed by the time the sample is drawn. Alternative methods could be to draw actual trough levels or to estimate $C_{\text {min }}$ using 
existing population PK models. However, these methods are not feasible in clinical practice because the timing of actual trough samples would be inconvenient for patients and the use of population PK models would be time consuming and would seriously delay the report of PK results and treatment advice to the treating physician and patient. Furthermore, Bayesian estimates of trough concentration based on a single sample suffer from shrinkage (regression to the mean), which will result in the misclassification of patients with low trough levels. Therefore, we chose to use this method, as it is easy to use, relatively precise, and thereby suitable to implement in routine care.

Many factors could contribute to low PK exposure, including drug-drug interactions, absorption problems (e.g. caused by poor bioavailability, food effects, or altered stomach $\mathrm{pH}$ ), pharmacogenetics, and compliance. ${ }^{43}$ Compliance can be defined as the extent to which the patient follows the dosing schedule as intended by the prescriber. Especially in case of long-term treatment, compliance is known to decrease over time, potentially leading to low PK exposure and thereby decreased efficacy. ${ }^{44}$ For example, poor adherence to imatinib has been related to suboptimal treatment outcomes. ${ }^{45}$ TDM could play a role in detecting poor compliance to oral anticancer drugs.

Previous attempts to evaluate the efficacy of TDM have failed to do so because of the unwillingness of treating physicians to follow treatment recommendations. ${ }^{8}$ We realize it is important that treatment recommendations should be followed to adequately evaluate the feasibility, tolerability, and efficacy of TDM. This is one of the reasons why physician adherence was chosen as one of the secondary objectives. We hope to achieve a high physician adherence by providing treating physicians with the available scientific evidence on exposure-efficacy relationships. Also, we summarize for them the number of patients previously treated at the proposed dose level (e.g. in the phase I study) and the tolerability in these patients.

We believe that the current fixed dosing paradigm should be changed. Subtherapeutic treatment with these expensive drugs due to low PK exposure at the standard dose is senseless. It is our opinion that personalized dosing based on individual drug levels is far more rational. If this large prospective study underscores the results of previous retrospective studies and prospective feasibility studies, ${ }^{1,2,6-8}$ this will support the implementation of PK-guided dose optimization as the new standard, although we realize that classical endpoints such as improvement of survival and/or quality of life will not be explored in this study. Because reimbursement of drug level measurement and administration of higher than approved doses of these expensive drugs could remain a challenge in the implementation of TDM as the standard of care in oncology, next steps would be to perform cost-effectiveness analyses and to address this with the concerning healthcare authorities. 
The DPOG-TDM study protocol has been developed to be a dynamic protocol, meaning that future oral anticancer drugs could be added to the protocol. Also, when new literature on exposure-efficacy relationships becomes available, the TDM targets could be updated. The guidance provided in this protocol could also be used outside this study for the implementation of TDM of oral anticancer drugs in the rest of the world.

In conclusion, this prospective clinical trial evaluating the feasibility, tolerability, and efficacy of TDM of oral anticancer drugs will be a valuable contribution to the fields of clinical pharmacology and oncology and holds promise to optimize treatment outcomes for patients treated with these agents. 


\section{REFERENCES}

1. Verheijen RB, Bins S, Mathijssen RHJ, Lolkema MP, van Doorn L, Schellens JHM et al. Individualized pazopanib dosing: a prospective feasibility study in cancer patients. Clin Cancer Res 2016; 22: 5738-5746.

2. Lankheet NAG, Kloth JSL, Gadellaa-van Hooijdonk CGM, Cirkel GA, Mathijssen RHJ, Lolkema MPJK et al. Pharmacokinetically guided sunitinib dosing: a feasibility study in patients with advanced solid tumours. Br J Cancer 2014; 110: 2441-2449.

3. Lankheet NAG, Knapen LM, Schellens JHM, Beijnen JH, Steeghs N, Huitema ADR. Plasma concentrations of tyrosine kinase inhibitors imatinib, erlotinib, and sunitinib in routine clinical outpatient cancer care. Ther Drug Monit 2014; 36: 326-34.

4. Verheijen RB, Yu H, Schellens JHM, Beijnen JH, Steeghs N, Huitema ADR. Practical recommendations for therapeutic drug monitoring of kinase inhibitors in oncology. Clin Pharmacol Ther 2017; 102: 765-776.

5. Groenland SL, van Nuland M, Verheijen RB, Schellens JHM, Beijnen JH, Huitema ADR et al. Therapeutic drug monitoring of oral anti-hormonal drugs in oncology. Clin Pharmacokinet 2019; 58: 299-308.

6. Lankheet NAG, Desar IME, Mulder SF, Burger DM, Kweekel DM, van Herpen CML et al. Optimizing the dose in cancer patients treated with imatinib, sunitinib and pazopanib. $\mathrm{Br} J$ Clin Pharmacol 2017; 83: 2195-2204.

7. Fox P, Balleine RL, Lee C, Gao B, Balakrishnar B, Menzies AM et al. Dose escalation of tamoxifen in patients with low endoxifen level: evidence for therapeutic drug monitoring the TADE study. Clin Cancer Res 2016; 22: 3164-3171.

8. Gotta V, Widmer N, Decosterd LA, Chalandon Y, Heim D, Gregor M et al. Clinical usefulness of therapeutic concentration monitoring for imatinib dosage individualization: results from a randomized controlled trial. Cancer Chemother Pharmacol 2014; 74: 1307-1319.

9. NCT01031628. Study of dose escalation versus no dose escalation of imatinib in metastatic gastrointestinal stromal tumors. Available from: http://clinicaltrials.gov/ct2/show/NCT01031628.

10. Koolen SLW, Bins S, Mathijssen RHJ. Individualized tamoxifen dose escalation - letter. Clin Cancer Res 2016; 22: 6300.

11. Carton E, Noe G, Huillard O, Golmard L, Giroux J, Cessot A et al. Relation between plasma trough concentration of abiraterone and prostate-specific antigen response in metastatic castration-resistant prostate cancer patients. Eur J Cancer 2017; 72: 54-61.

12. Food and Drug Administration. Center for Drug Evaluation and Research. Alectinib Clinical Pharmacology and Biopharmaceutics Review. 2016. Available from: http://www.accessdata.fda.gov/drugsatfda_docs/nda/2015/208434Orig1s000ClinPharmR.p df.

13. Rini BI, Garrett M, Poland B, Dutcher JP, Rixe O, Wilding G et al. Axitinib in metastatic renal cell carcinoma: results of a pharmacokinetic and pharmacodynamic analysis. J Clin Pharmacol 2013; 53: 491-504.

14. Hsyu P-H, Mould DR, Upton RN, Amantea M. Pharmacokinetic-pharmacodynamic relationship of bosutinib in patients with chronic phase chronic myeloid leukemia. Cancer 
Chemother Pharmacol 2013; 71: 209-18.

15. Food and Drug Administration. Center for Drug Evaluation and Research. Cobimetinib Clinical Pharmacology and Biopharmaceutics Review. 2014. Available from: http://www.accessdata.fda.gov/drugsatfda_docs/nda/2015/206192Orig1s000ClinPharmR.p df.

16. Food and Drug Administration. Center for Drug Evaluation and Research. Crizotinib Clinical Pharmacology and Biopharmaceutics Review. 2011. Available from: http://www.accessdata.fda.gov/drugsatfda_docs/nda/2011/2025700rig1s000ClinPharmR.p df.

17. Wang X, Roy A, Hochhaus A, Kantarjian HM, Chen T-T, Shah NP. Differential effects of dosing regimen on the safety and efficacy of dasatinib: retrospective exposure-response analysis of a Phase III study. Clin Pharmacol Adv Appl 2013; 5: 85-97.

18. Gibbons JA, Ouatas T, Krauwinkel W, Ohtsu Y, van der Walt JS, Beddo V et al. Clinical pharmacokinetic studies of enzalutamide. Clin Pharmacokinet 2015; 54: 1043-1055.

19. Ravaud A, Urva SR, Grosch K, Cheung WK, Anak O, Sellami DB. Relationship between everolimus exposure and safety and efficacy: meta-analysis of clinical trials in oncology. Eur J Cancer 2014; 50: 486-495.

20. Zhao Y-Y, Li S, Zhang Y, Zhao H-Y, Liao H, Guo Y et al. The relationship between drug exposure and clinical outcomes of non-small cell lung cancer patients treated with gefitinib. Med Oncol 2011; 28: 697-702.

21. Giles FJ, Yin OQP, Sallas WM, le Coutre PD, Woodman RC, Ottmann OG et al. Nilotinib population pharmacokinetics and exposure-response analysis in patients with imatinibresistant or -intolerant chronic myeloid leukemia. Eur J Clin Pharmacol 2013; 69: 813-823.

22. Fong P, Boss D, Yap T, Tutt A, Wu P, Mergui-Roelvink M et al. Inhibition of poly(ADP-ribose) polymerase in tumors from BRCA mutation carriers. N Engl J Med 2009; 361: 557-568.

23. Food and Drug Administration. Center for Drug Evaluation and Research. Clinical Pharmacology and Biopharmaceutics Review Palbociclib. 2014. Available from: https://www.accessdata.fda.gov/drugsatfda_docs/nda/2015/2071030rig1s000ClinPharmR. pdf.

24. Suttle AB, Ball HA, Molimard M, Hutson TE, Carpenter C, Rajagopalan D et al. Relationships between pazopanib exposure and clinical safety and efficacy in patients with advanced renal cell carcinoma. Br J Cancer 2014; 111: 1-8.

25. Verheijen RB, Swart LE, Beijnen JH, Schellens JHM, Huitema ADR, Steeghs N. Exposuresurvival analyses of pazopanib in renal cell carcinoma and soft tissue sarcoma patients: opportunities for dose optimization. Cancer Chemother Pharmacol 2017; 80: 1171-1178.

26. Regorafenib Clinical Pharmacology and Biopharmaceutics Review. 2012. Available from: http://www.accessdata.fda.gov/drugsatfda_docs/nda/2012/203085Orig1s000ClinPharmR.p df.

27. Minami H, Kawada K, Ebi H, Kitagawa K, Kim Y Il, Araki K et al. Phase I and pharmacokinetic study of sorafenib, an oral multikinase inhibitor, in Japanese patients with advanced refractory solid tumors. Cancer Sci 2008; 99: 1492-1498.

28. Madlensky L, Natarajan L, Tchu S, Pu M, Mortimer J, Flatt SW et al. Tamoxifen metabolite concentrations, CYP2D6 genotype, and breast cancer outcomes. Clin Pharmacol Ther 2011; 
89: 718-25.

29. Flaherty KT, Robert C, Hersey P, Nathan PD, Garbe C, Milhem M et al. Improved survival with MEK Inhibition in BRAF-mutated melanoma. N Engl J Med 2012; 367: 107-114.

30. Nijenhuis CM, Huitema ADR, Blank C, Haanen JBAG, van Thienen JV, Rosing $\mathrm{H}$ et al. Clinical pharmacokinetics of vemurafenib in BRAF-mutated melanoma patients. J Clin Pharmacol 2016; 57: 125-128.

31. Food and Drug Administration. Center for Drug Evaluation and Research. Vismodegib Clinical Pharmacology and Biopharmaceutics Review. 2012. Available from: http://www.accessdata.fda.gov/drugsatfda_docs/nda/2012/2033880rig1s000ClinPharmR.p df.

32. Willemsen AEACB, Lubberman FJE, Tol J, Gerritsen WR, van Herpen CML, van Erp NP. Effect of food and acid-reducing agents on the absorption of oral targeted therapies in solid tumors. Drug Discov Today 2016; 21: 962-976.

33. Yu H, van Erp NP, Bins S, Mathijssen RHJ, Schellens JHM, Beijnen JH et al. Development of a pharmacokinetic model to describe the complex pharmacokinetics of pazopanib in cancer patients. Clin Pharmacokinet 2017; 56: 293-303.

34. Herbrink M, de Vries N, Rosing H, Huitema ADR, Nuijen B, Schellens JHM et al. Quantification of 11 therapeutic kinase inhibitors in human plasma for therapeutic drug monitoring using liquid chromatography coupled with tandem mass spectrometry. Ther Drug Monit 2016; 38: 649-656.

35. Herbrink M, de Vries N, Rosing H, Huitema ADR, Nuijen B, Schellens JHM et al. Development and validation of a liquid chromatography - tandem mass spectrometry analytical method for the therapeutic drug monitoring of eight novel anticancer drugs. Biomed Chromatogr 2018; 32: 1-9.

36. van Nuland M, Hillebrand MJX, Rosing $H$, Schellens JHM, Beijnen JH. Development and validation of an LC-MS/MS method for the simultaneous quantification of abiraterone, enzalutamide, and their major metabolites in human plasma. Ther Drug Monit 2017; 39: 243251.

37. de Krou S, Rosing H, Nuijen B, Schellens JHM, Beijnen JH. Fast and adequate liquid chromatography-tandem mass spectrometric determination of Z-endoxifen serum levels for therapeutic drug monitoring. Ther Drug Monit 2017; 39: 132-137.

38. Binkhorst L, Mathijssen RHJ, Moghaddam-helmantel IMG, de Bruijn P, van Gelder T, Wiemer EAC et al. Quantification of tamoxifen and three of its phase-I metabolites in human plasma by liquid chromatography / triple-quadrupole mass spectrometry. J Pharm Biomed Anal 2011; 56: 1016-1023.

39. de Bruijn P, Sleijfer S, Lam M, Mathijssen RHJ, Wiemer EAC, Loos WJ. Bioanalytical method for the quantification of sunitinib and its N-desethyl metabolite SU12662 in human plasma by ultra performance liquid chromatography / tandem triple-quadrupole mass spectrometry. J Pharm Biomed Anal 2010; 51: 934-941.

40. Wang Y, Chia Y, Nedelman J, Schran H, Mahon F, Molimard M. A therapeutic drug monitoring algorithm for refining the imatinib trough level obtained at different sampling times. Ther Drug Monit 2009; 31: 579-584.

41. R Core Development Team. A language and environment for statistical computing. R Found 
Statistical Computing, Vienna. 2016. Available from: https://www.r-project.org/.

42. Mathijssen RHJ, de Jong F, Loos W, van der Bol J, Verweij J, Sparreboom A. Flat-fixed dosing versus body surface area-based dosing of anticancer drugs in adults: does it make a difference? Oncologist 2007; 12: 913-923.

43. Hussaarts KGAM, Veerman GDM, Jansman FGA, van Gelder T, Mathijssen RHJ, van Leeuwen RWF. Clinically relevant drug interactions with multikinase inhibitors: a review. Ther Adv Med Oncol 2019; 11: 1-34.

44. Cardoso E, Csajka C, Schneider MP, Widmer N. Effect of adherence on pharmacokinetic/pharmacodynamic relationships of oral targeted anticancer drugs. Clin Pharmacokinet 2017; 57: 1-6.

45. Noens $L$, van Lierde $M$, de Bock $R$, Verhoef $G$, Zachée $P$, Martiat $P$ et al. Prevalence, determinants, and outcomes of nonadherence to imatinib therapy in patients with chronic myeloid leukemia: the ADAGIO study. Blood 2009; 113: 5401-5411. 


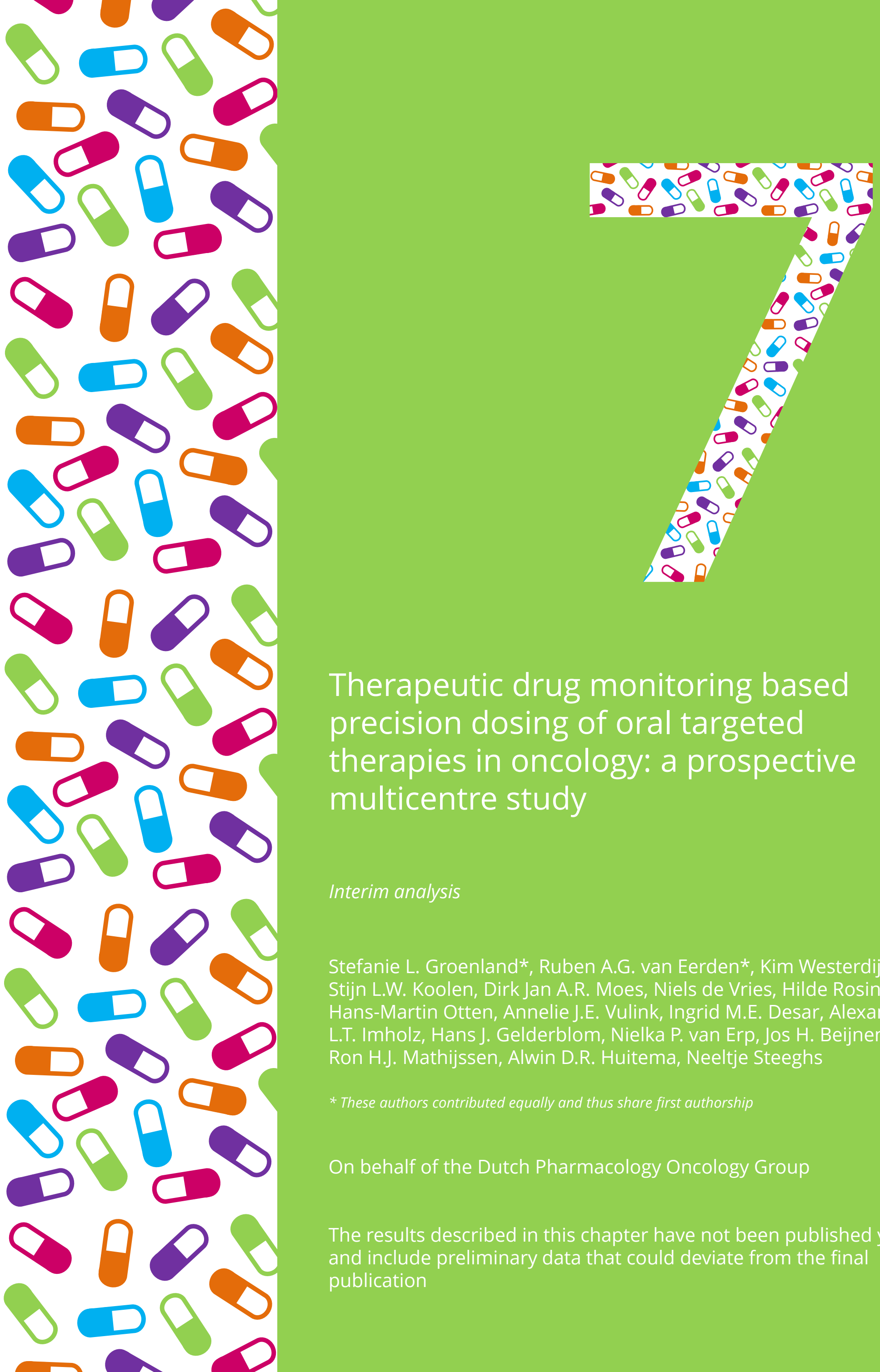

Therapeutic drug monitoring based precision dosing of oral targeted therapies in oncology: a prospective multicentre study

Interim analysis

Stefanie L. Groenland*, Ruben A.G. van Eerden*, Kim Westerdijk, Stijn L.W. Koolen, Dirk Jan A.R. Moes, Niels de Vries, Hilde Rosing, Hans-Martin Otten, Annelie J.E. Vulink, Ingrid M.E. Desar, Alexander L.T. Imholz, Hans J. Gelderblom, Nielka P. van Erp, Jos H. Beijnen, Ron H.J. Mathijssen, Alwin D.R. Huitema, Neeltje Steeghs

* These authors contributed equally and thus share first authorship

On behalf of the Dutch Pharmacology Oncology Group

The results described in this chapter have not been published yet and include preliminary data that could deviate from the final publication 



\title{
ABSTRACT
}

\begin{abstract}
Aim
Higher imatinib exposure is correlated with longer time to progression, while the variability in exposure is high. This provides a strong rationale for therapeutic drug monitoring, which has therefore been implemented in routine clinical practice in our institute. The aim of this study is to evaluate whether pharmacokinetically (PK)-guided dose increases are feasible in daily clinical practice and result in an improved exposure $\left(C_{\min } \geq 1100 \mathrm{ng} / \mathrm{mL}\right)$ and longer progression-free survival (PFS).
\end{abstract}

\section{Methods}

This retrospective study included all patients with a gastrointestinal stromal tumour (GIST) in the Netherlands Cancer Institute who started imatinib treatment at a dose of $400 \mathrm{mg}$ and of whom PK plasma samples were available. Of these patients, minimum plasma concentrations (Cmin) of imatinib, frequency and successfulness of PK-guided dose increases and PFS in the palliative treatment setting were analysed.

\section{Results}

In total, 169 consecutive patients were included, of whom 1402 PK samples were collected. In 126 patients (75\%), $C_{\min }$ was below the efficacy threshold of $1100 \mathrm{ng} / \mathrm{mL}$. In 78 of these patients (62\%), a PK-guided dose increase was performed, which was successful in 49 patients (63\%). PFS was similar in patients with and without imatinib dose increase. However, due to the small number of patients with progressive disease, no definite conclusions on the effect on PFS could yet be drawn.

\section{Conclusion}

This is the largest cohort evaluating PK-guided dose increases of imatinib in patients with GIST in routine clinical practice and demonstrating its feasibility. PK-guided dose increases should be applied to optimise exposure in the significant subset of patients with a low $C_{\min }$. 


\section{INTRODUCTION}

Gastrointestinal stromal tumour (GIST) is a rare type of soft tissue sarcoma treated with the tyrosine kinase inhibitor imatinib in neoadjuvant, adjuvant and palliative treatment setting. Since the introduction of imatinib, the survival in advanced GIST has improved drastically. ${ }^{1}$ Nevertheless, therapeutic failures are still common and survival could be further improved.

In a previous study by Demetri et al. the time to progression was almost three times longer for patients with imatinib trough levels $\left(C_{\min }\right) \geq 1100 \mathrm{ng} / \mathrm{mL}$ compared with those with a $C_{\text {min }}$ below this threshold (30 months vs. 11 months). ${ }^{2}$ These results are in line with the exposure-response relationship that has been identified for patients with chronic myeloid leukaemia $(\mathrm{CML})$ treated with imatinib. ${ }^{3-5}$ There is, however, a large inter- and intraindividual variability $\left(47-75 \%\right.$ and $19-26 \%$, respectively) in imatinib exposure. ${ }^{2,6-10}$ Furthermore, decreased imatinib exposure of $30 \%$ after long-term treatment has been described. ${ }^{8}$ The high variability in imatinib exposure combined with the clear exposureresponse relationship provides a strong rationale for adjusting the dose based on measured drug levels, also known as therapeutic drug monitoring (TDM).

Previously, our group has published a report describing the pharmacokinetics (PK) of imatinib in routine clinical care, showing that $32-73 \%$ of the patients was systematically underexposed. ${ }^{9,11}$ In addition, TDM of imatinib in patients with GIST has shown promising results. PK-guided dose interventions in 20 patients treated with imatinib resulted in adequate $C_{\min }(1100-3200 \mathrm{ng} / \mathrm{mL})$ in $95 \%$ of patients in a previous study. ${ }^{10}$ In addition, Zuidema et al. described that TDM-guided dosing is a cost-effective intervention for patients with advanced GIST. ${ }^{12}$ Based on the previous fact, TDM of imatinib has been implemented as standard of care in the Netherlands Cancer Institute (NKI).

In our routine TDM program, drug levels are measured regularly in the out-patient clinic and dose regimens are adjusted accordingly, striving for $a C_{\min } \geq 1100 \mathrm{ng} / \mathrm{mL}$. 2,3,7 Data on the state of affairs of TDM of imatinib in patients with GIST are not widely available. Therefore, the aim of this retrospective study was to evaluate whether PK-guided dose increases are feasible in daily clinical practice and result in an increased proportion of patients with adequate exposure (as defined as $C_{\min } \geq 1100 \mathrm{ng} / \mathrm{mL}$ ) and longer progression-free survival (PFS).

\section{METHODS}

\section{Patient selection}

Data were collected from the Dutch GIST Registry (DGR), a database containing information on all patients with GIST treated in one of the five Dutch GIST centres since 2009. The DGR was approved by the local independent ethics committee. All patients in 
the $\mathrm{NKI}$, one of these centres, who started treatment with imatinib at the standard dose of $400 \mathrm{mg}$ once daily (QD) and of whom PK samples were available were included. Of these patients, demographic data, tumour characteristics, imatinib treatment details, imatinib trough levels, PFS (in case of metastatic disease) and clinically relevant toxicities (i.e. toxicities requiring a dose reduction, treatment interruption or discontinuation) were obtained from the database. In the neoadjuvant setting, imatinib was continued until maximum tumour response (in general around 6 months). ${ }^{13,14}$ The duration of imatinib treatment in the adjuvant setting was 12 months before 2012 and was expanded to 36 months thereafter. ${ }^{15,16}$ Palliative treatment was continued until progressive disease or unacceptable toxicity.

\section{PK analyses}

Plasma samples were collected during routine follow-up visits (in general, monthly during the first three months and once every three months thereafter). Date and time of last imatinib ingestion and plasma collection were recorded. Imatinib concentrations were measured using a validated liquid chromatography-tandem mass spectrometry assay. ${ }^{17}$ $C_{\min }$ was estimated using the following formula, which has previously been validated by Wang et al. ${ }^{18}$ :

$$
C_{\text {min }}=C_{\text {measured }} * 0.5^{\frac{\text { dosing interval-TAD }}{t_{1 / 2}}}
$$

In this formula, the $C_{\min }$ is the calculated minimum plasma concentration (trough level, in $\mathrm{ng} / \mathrm{mL}$ ), $\mathrm{C}_{\text {measured }}$ is the measured plasma concentration (in $\mathrm{ng} / \mathrm{mL}$ ), dosing interval is the time in hours between two consecutive ingestions of the drug, TAD is the time after dose, that is, the time between last administration of imatinib and collection of the plasma sample in hours and $t_{1 / 2}$ is the elimination half-life of the drug (18 hours for imatinib $\left.{ }^{19}\right)$.

If samples were collected within 0.5 hour after imatinib intake, they were interpreted as $C_{\min }$. When samples were collected before $t_{\max }$ (i.e. 0.5-2.5 hours after intake), $C_{\min }$ was calculated by multiplying the ratio of the measured concentration and the typical population concentration (at the corresponding time after dose) with the typical population value of $\mathrm{C}_{\min }{ }^{20}$

\section{TDM subgroups}

Patients were retrospectively divided into group 1 (low PK) if they had a $C_{\text {min }}$ below the threshold of $1100 \mathrm{ng} / \mathrm{mL}$ on at least two occasions or if they received a PK-guided dose increase based on one $C_{\text {min }}$ below this threshold. Patients in group 1 were further subdivided based on the experienced toxicity and whether or not they received a dose increase (group 1A: no toxicity, dose increase; group 1B: toxicity, no dose increase; group 1C: no toxicity, no dose increase). Dose increases were considered successful if the median $C_{\min }$ at the increased dose was $\geq 1100 \mathrm{ng} / \mathrm{mL}$ and if the patient tolerated this dose for at least six months. Patients were divided into group 2 (adequate PK) if all $C_{\text {min }}$ were $\geq$ 
$1100 \mathrm{ng} / \mathrm{mL}$. Patients in group 2 were further subdivided based on experienced toxicity (group 2A: no toxicity; group 2B: toxicity). For the comparison of exposure and PFS, group $1 \mathrm{~B}+1 \mathrm{C}$ (low $\mathrm{PK}$, no dose increase) and group 2A+2B (adequate PK) were combined and compared with group $1 \mathrm{~A}$ (low PK, dose increase).

\section{Statistical analysis}

Descriptive statistics were performed using IBM SPSS Statistics, version 25, and PK results were analysed using R, version 3.6.1 (R Project, Vienna, Austria). Survival was estimated with the Kaplan-Meier method. To compare treatment outcomes, PFS was compared for metastatic patients with KIT exon 11 mutations using Cox regression analyses. Factors with a known correlation to outcome (according to National Institutes of Health risk assessment: tumour size, primary tumour location and mitotic rate ${ }^{21}$ and World Health Organisation performance status ${ }^{22}$ ) and with an apparent unequal distribution among the subgroups were included in the multivariable analysis. A two-sided $P$-value $<0.05$ was considered statistically significant.

\section{RESULTS}

\section{Patient characteristics}

In total, 202 patients were diagnosed with GIST between January 2009 and July 2019, registered in the DGR and treated in the NKI. PK data were available for 169 patients (84\%). Sixteen patients were treated in the beginning of the implementation and received no TDM, thirteen patients just started treatment with no PK samples yet and four patients were lost to follow-up before PK data were collected. Patient characteristics are shown in Table 1. The median age at diagnosis was 63 years (interquartile range (IQR): $53-70$ ) and a slight majority was men (58\%). Most frequent GIST location was the stomach (50\%) or ileum/jejunum (34\%). The most common mutation was KIT exon 11 (82\%) but also 13 patients had a known imatinib insensitive mutation: 11 KIT exon 9 and 2 PDGFR D842V. At baseline, $39 \%$ of the tumours had a high mitotic rate and $27 \%$ of the patients already had metastatic disease. Patients were treated in different treatment settings: neoadjuvant $(n=75)$, adjuvant $(n=75)$ and palliative $(n=83)$. Patients who received a dose increase (group $1 \mathrm{~A})$ appeared to be younger, and patients with a low exposure (group 1) were relatively more often men (68\% and $54 \%$ vs. $44 \%$ in group 2 ) and had KIT exon 11 mutated GIST ( $86 \%$ and $90 \%$ vs. $65 \%$ in group 2 ).

\section{PK samples}

In total, 1402 PK samples were collected with a median of 7 samples per patient (IQR: 412). The median time after dose was 15.3 hours (IQR: 10.0-18.5 hours). The median total time on imatinib was 23 months (IQR: 10-36 months). The median $C_{\text {min }}$ per patient was 1074 ng/mL (IQR: $946-1247$ ng/mL, Figure 1). Inter- and intra-individual variability was 49\% 
and 26\%, respectively, at the standard dose of $400 \mathrm{mg}$ QD. Table 2 provides an overview of all PK samples.

Table 1 - Patient characteristics

\begin{tabular}{|c|c|c|c|c|}
\hline & All patients & Group 1A & $\begin{array}{l}\text { Group } \\
1 \mathrm{~B}+1 \mathrm{C}\end{array}$ & $\begin{array}{l}\text { Group } \\
2 A+2 B\end{array}$ \\
\hline No. of patients & 169 & 78 & 48 & 43 \\
\hline Age at diagnosis & $63[53-70]$ & $58[50-68]$ & $65[57-73]$ & $64[60-75]$ \\
\hline $\begin{array}{l}\text { Primary tumour size } \\
\text { (in } \mathrm{mm} \text { ) }\end{array}$ & $100[62-150]$ & 102 [64-135] & 96 [60-150] & $108[63-168]$ \\
\hline Gender, male & $98(58 \%)$ & $53(68 \%)$ & $26(54 \%)$ & $19(44 \%)$ \\
\hline \multicolumn{5}{|l|}{ Primary GIST location } \\
\hline Gastric & $84(50 \%)$ & $34(44 \%)$ & $26(54 \%)$ & $24(56 \%)$ \\
\hline Ileum/jejunum & $57(34 \%)$ & $29(37 \%)$ & $16(33 \%)$ & $12(28 \%)$ \\
\hline Duodenum & $13(8 \%)$ & $8(10 \%)$ & $2(4 \%)$ & $3(7 \%)$ \\
\hline Rectum & $7(4 \%)$ & $4(5 \%)$ & $1(2 \%)$ & $2(5 \%)$ \\
\hline Esophagus & $3(2 \%)$ & $1(1 \%)$ & $2(4 \%)$ & 0 \\
\hline Colon & $3(2 \%)$ & $1(1 \%)$ & $1(2 \%)$ & $1(2 \%)$ \\
\hline Other ${ }^{\mathrm{a}}$ & $2(1 \%)$ & $1(1 \%)$ & 0 & $1(2 \%)$ \\
\hline \multicolumn{5}{|l|}{ Mutation status } \\
\hline KIT exon 11 & $138(82 \%)$ & $67(86 \%)$ & $43(90 \%)$ & $28(65 \%)$ \\
\hline KIT exon 9 & $11(7 \%)$ & $5(6 \%)$ & 0 & $6(14 \%)$ \\
\hline KIT other & $2(1 \%)$ & $1(1 \%)$ & $1(2 \%)$ & 0 \\
\hline PDGFR non-D842V & $6(4 \%)$ & $3(4 \%)$ & $2(4 \%)$ & $1(2 \%)$ \\
\hline PDGFR D842V & $2(1 \%)$ & 0 & $2(4 \%)$ & 0 \\
\hline SDH deficient & $1(1 \%)$ & 0 & 0 & $1(2 \%)$ \\
\hline NF1 related & $2(1 \%)$ & 0 & 0 & $2(5 \%)$ \\
\hline Wildtype & $4(2 \%)$ & $1(1 \%)$ & 0 & $3(7 \%)$ \\
\hline No mutation analysis & $3(2 \%)$ & $1(1 \%)$ & 0 & $2(5 \%)$ \\
\hline \multicolumn{5}{|l|}{ Baseline mitotic rate } \\
\hline $\operatorname{Low}\left(\leq 5 / 5 \mathrm{~mm}^{\mathrm{b}}\right)$ & $84(50 \%)$ & $39(50 \%)$ & $27(56 \%)$ & $18(42 \%)$ \\
\hline High $\left(>5 / 5 \mathrm{~mm}^{\mathrm{b}}\right)$ & $66(39 \%)$ & $34(44 \%)$ & $16(33 \%)$ & $16(37 \%)$ \\
\hline Unknown & $19(11 \%)$ & $5(6 \%)$ & $5(10 \%)$ & $9(21 \%)$ \\
\hline \multicolumn{5}{|l|}{$\begin{array}{l}\text { Tumour status at } \\
\text { diagnosis }\end{array}$} \\
\hline Localised disease & $55(33 \%)$ & $27(35 \%)$ & $17(35 \%)$ & $11(26 \%)$ \\
\hline Locally advanced & $64(38 \%)$ & $30(39 \%)$ & $17(35 \%)$ & $17(40 \%)$ \\
\hline Multiple locations & $4(2 \%)$ & $1(1 \%)$ & $1(2 \%)$ & $2(5 \%)$ \\
\hline Metastatic disease & $46(27 \%)$ & $20(26 \%)$ & $13(27 \%)$ & $13(30 \%)$ \\
\hline \multicolumn{5}{|l|}{ Performance status $^{c}$} \\
\hline WHO 0 & $117(69 \%)$ & $55(71 \%)$ & $32(67 \%)$ & $30(70 \%)$ \\
\hline WHO 1 & $31(18 \%)$ & $10(13 \%)$ & $14(29 \%)$ & $7(16 \%)$ \\
\hline $\mathrm{WHO} \geq 2$ & $3(2 \%)$ & $2(2 \%)$ & 0 & $1(2 \%)$ \\
\hline Unknown & $18(11 \%)$ & $11(14 \%)$ & $2(4 \%)$ & $5(12 \%)$ \\
\hline \multicolumn{5}{|l|}{ Treatment objective $^{d}$} \\
\hline Neoadjuvant & $75(32 \%)$ & $33(30 \%)$ & $22(33 \%)$ & $20(36 \%)$ \\
\hline Adjuvant & 75 (32\%) & $41(37 \%)$ & $22(33 \%)$ & $12(22 \%)$ \\
\hline Palliative & $83(36 \%)$ & $37(33 \%)$ & $23(34 \%)$ & $23(42 \%)$ \\
\hline
\end{tabular}




\begin{tabular}{ccccc}
\hline Treatment duration $^{\mathbf{e}}$ & & & & \\
\hline Neoadjuvant & $6[5-7]$ & $6[5-7]$ & $7[6-8]$ & $6[4-8]$ \\
\hline Adjuvant & $34[31-36]$ & $35[34-36]$ & $30[29-31]$ & $23[10-36]$ \\
\hline Palliative & $30[23-37]$ & $35[13-57]$ & $30[28-32]$ & $12[8-16]$ \\
\hline
\end{tabular}

Data are expressed as no. (\%) or median [interquartile range], as appropriate. Group 1A: low exposure, PK-guided dose increase, group 1B+1C: low exposure, no PK-guided dose increase, group 2A+2B: adequate exposure all the time.

${ }^{a}$ One in the liver, one deposition in mesentery

${ }^{\mathrm{b}}$ One KIT exon 13 and one KIT exon 17

cWHO Performance status at start of first treatment or if palliative treatment at start of palliative treatment

d Several patients had imatinib in multiple treatment settings

e Median treatment duration (in months, [95\% confidence interval]) was estimated using the Kaplan-Meier method, censoring patients who were still on treatment.

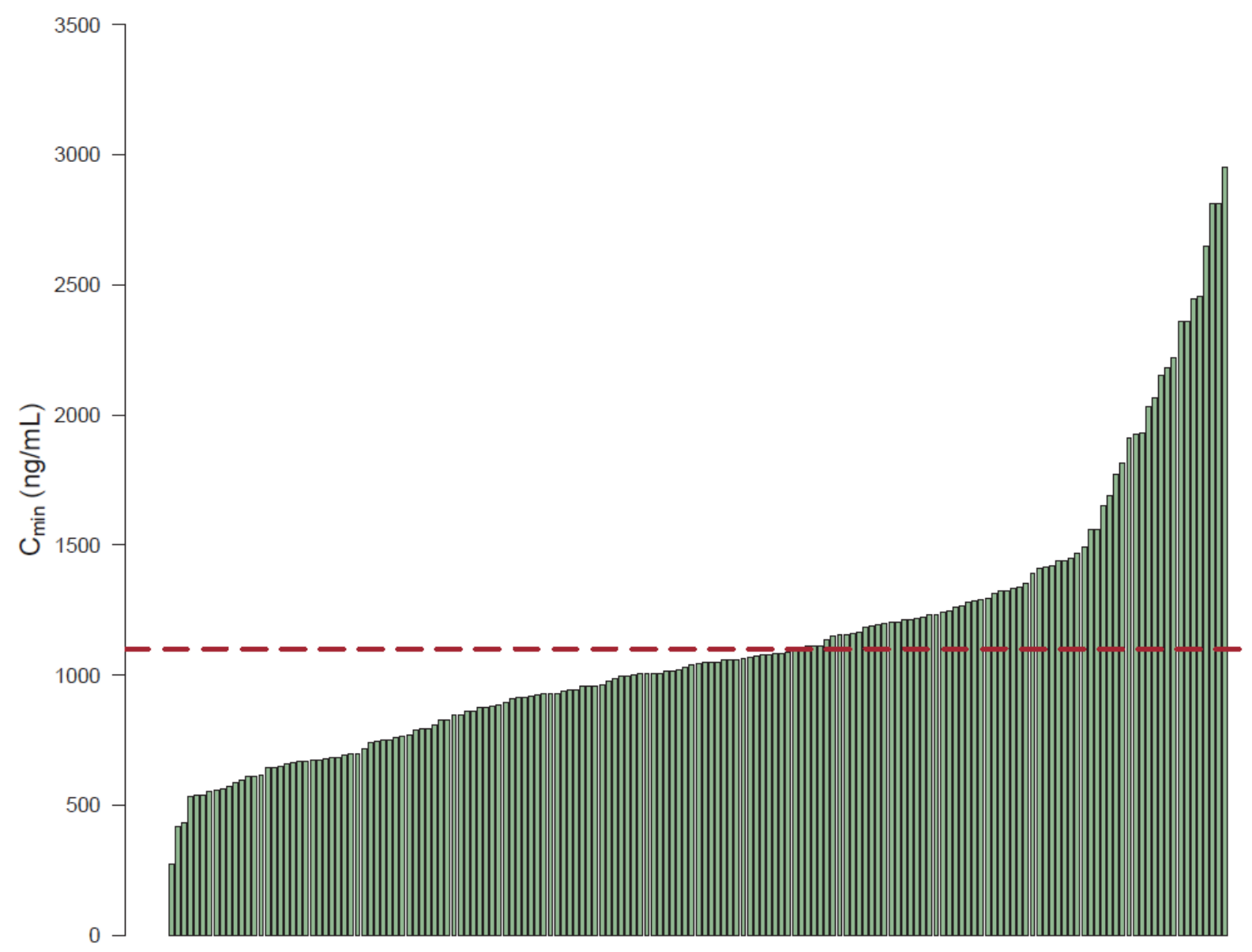

Figure 1 - Median imatinib $\mathrm{C}_{\min }$ per patient at the standard dose

Each bar represents one patient. The dashed line indicates the efficacy threshold of $1100 \mathrm{ng} / \mathrm{mL}$. Only PK samples at the standard dose of $400 \mathrm{mg}$ QD were included. Ninety-eight patients (58\%) had a median $C_{\min }$ below this threshold. Inter- and intra-individual variability were $49 \%$ and $26 \%$, respectively.

$C_{\min }=$ minimum plasma concentration; $P K=$ pharmacokinetic; $Q D=$ once daily 


\section{TDM recommendations}

In total, 126 patients (75\%) had a low imatinib exposure according to the used definition (group 1: $\geq 2 C_{\min }<1100 \mathrm{ng} / \mathrm{mL}$ or a dose increase based on a single $C_{\min }$ below this threshold), while in the other 43 patients (25\%), $C_{\min }$ was $\geq 1100 \mathrm{ng} / \mathrm{mL}$ all the time (group 2). Figure 2 demonstrates the distribution of patients among the different groups.

In 78 of the patients with a low PK exposure (66\%), a PK-guided dose increase was performed (group 1A) after a median of two low PK samples (IQR: 1-3). The daily dose of imatinib was escalated to $600 \mathrm{mg}$ in 43 patients, while in 35 patients, the dose was further increased to $800 \mathrm{mg}$ (either administered as $800 \mathrm{mg}$ QD or $400 \mathrm{mg}$ twice daily). This PKguided dose increase was successful (i.e. target attainment without additional toxicities) in 49 of these patients (63\%). The $C_{\text {min }}$ increased by 55\% (IQR: $35-76 \%$ ) and 86\% (IQR: 65$127 \%$ ) with dose increases to $600 \mathrm{mg}$ and $800 \mathrm{mg}$, respectively. Reasons why this was not successful in other patients were emerging toxicity $(n=13)$, PK (i.e. $C_{\min }$ remained below target, $n=7$ ) or both $(n=2)$. In addition, in seven patients, the effect has not been evaluated (yet). The median imatinib $C_{\text {min }}$ increased from $864 \mathrm{ng} / \mathrm{mL}$ (IQR: 698-1089 ng/mL) before to $1198 \mathrm{ng} / \mathrm{mL}$ (IQR: $1081-1428 \mathrm{ng} / \mathrm{mL}$ ) after the PK-guided dose increase $(p<0.001)$. Figure 3 visualizes imatinib exposure in the different subgroups in box plots.

In 23 patients with a low exposure (18\%), it was not feasible to perform a PK-guided dose increase, because they already experienced toxicity at the standard dose of $400 \mathrm{mg}$ QD (group 1B).

The remaining 25 patients with a low exposure (20\%) experienced no toxicities and a PKguided dose increase could thus theoretically have been implemented in these patients (group 1C). Reasons why PK-guided dose increases were not performed were physician adherence $\left(n=9\right.$; either due to borderline low $C_{\min }(n=2)$ or unknown reasons $\left.(n=7)\right)$, treatment discontinuation $(n=7)$, inclusion in a study where imatinib dose escalation was not allowed ( $n=3$, control arm (i.e. imatinib only) of a clinical trial investigating alternating treatment with imatinib and regorafenib) or the $C_{\min }$ was reported as adequate by the hospital pharmacist $(n=6)$.

In group 2, 26 patients (60\%) did not experience toxicities and continued treatment at the standard dose (group 2A), while 17 patients (40\%) did experience clinically relevant toxicities (group 2B). These toxicities resulted in imatinib dose reduction $(n=8)$, treatment interruption ( $n=5)$ and treatment discontinuation $(n=4)$. The median $C_{\min }$ was comparable in group $2 \mathrm{~A}$ and $2 \mathrm{~B}$. 
Table 2 - Pharmacokinetic characteristics of imatinib trough levels

\begin{tabular}{lcccc}
\hline & All patients & Group 1A & Group 1B+1C & Group 2A+2B \\
\hline No. of patients & 169 & 78 & 48 & 43 \\
\hline $\boldsymbol{C}_{\min }$ per patient $(\mathrm{ng} / \mathrm{mL})$ & $\begin{array}{c}1074 \\
{[946-1247]}\end{array}$ & $\begin{array}{c}1051 \\
{[902-1191]}\end{array}$ & $\begin{array}{c}1050 \\
{[950-1158]}\end{array}$ & $\begin{array}{c}1418 \\
{[1237-2813]}\end{array}$ \\
\hline $\begin{array}{l}\boldsymbol{C}_{\min } \text { per patient before PK- } \\
\text { guided dose increase }(\mathrm{ng} / \mathrm{mL})\end{array}$ & $\mathrm{NA}$ & $\begin{array}{c}864 \\
{[698-1089]^{\mathrm{a}}}\end{array}$ & $\mathrm{NA}$ & $\mathrm{NA}$ \\
\hline $\begin{array}{l}\mathbf{C}_{\min } \text { per patient after } \mathbf{P K}- \\
\text { guided dose increase }(\mathrm{ng} / \mathrm{mL})\end{array}$ & $\mathrm{NA}$ & $\begin{array}{c}1198 \\
{[1081-1428]^{\mathrm{a}}}\end{array}$ & $\mathrm{NA}$ & $\mathrm{NA}$ \\
\hline $\begin{array}{l}\text { Number of samples per } \\
\text { patient }\end{array}$ & $7[4-12]$ & $10[6-13]$ & $8[5-13]$ & $4[2-7]$ \\
\hline
\end{tabular}

Data are expressed as no. (\%) or median [interquartile range], as appropriate.

Group 1A: low exposure, PK-guided dose increase, group 1B+1C: low exposure, no PK-guided dose increase, group $2 \mathrm{~A}+2 \mathrm{~B}$ : adequate exposure all the time.

${ }^{a} C_{\min }$ after PK-guided dose increase was significantly higher than $C_{\min }$ before PK-guided dose increase ( $p<0.001$, Wilcoxon signed rank test). Patients in whom the effect of the PK-guided dose increase was not evaluated (yet) were excluded for this analysis.

$C_{\text {min }}=$ minimum plasma concentration; $N A=$ not applicable; $P K=$ pharmacokinetic

ALL IMATINIB PATIENTS: starting at $\mathbf{4 0 0} \mathrm{mg}$ and with available PK data $(\mathrm{n}=169)$

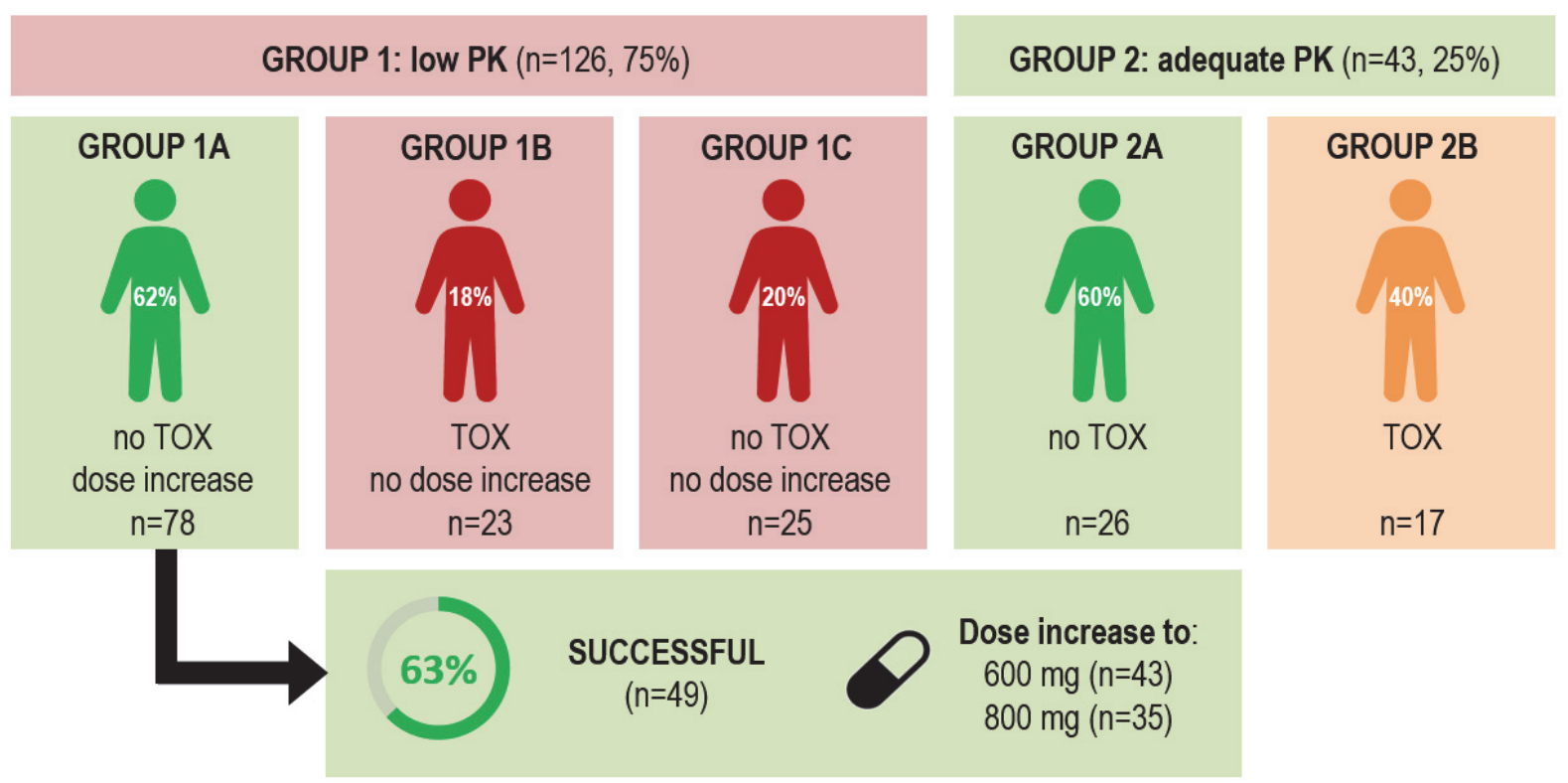

Figure 2 - Schematic overview of results

Patients were classified in group 1 if they had $\geq 2 C_{\min }$ below the efficacy threshold of $1100 \mathrm{ng} / \mathrm{mL}$ or if they received a PK-guided dose increase based on a single $C_{\min }$ below this threshold.

Patients in group 1 were further divided based on the experienced toxicity and whether or not they received a dose increase (group 1A: no toxicity, dose escalation; group 1B: toxicity, no dose increase; group 1C: no toxicity, no dose increase).

Dose increases were considered successful if the median $C_{\min }$ at the increased dose was $\geq 1100$ $\mathrm{ng} / \mathrm{mL}$ and if the patient tolerated this dose for at least six months.

Patients were classified in group 2 (adequate PK) if they had all $C_{\min } \geq 1100 \mathrm{ng} / \mathrm{mL}$.

Patients in group 2 were further divided based on the experienced toxicity (group 2A: no toxicity; group 2B: toxicity).

TOX = toxicity; $C_{\min }=$ minimum plasma concentration; $P K=$ pharmacokinetic 


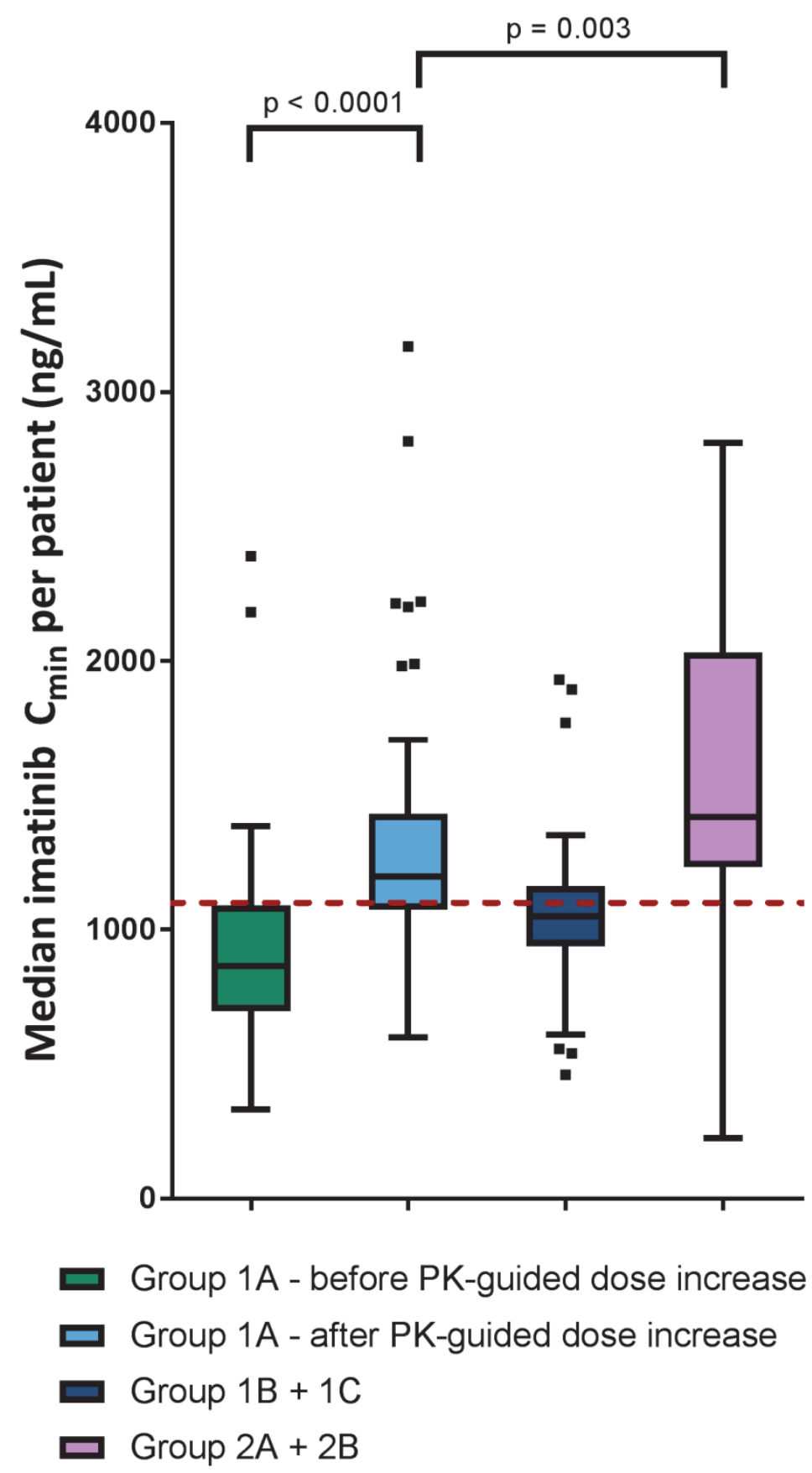

Figure 3 - Boxplots of imatinib $C_{\min }$ in patients with adequate and low exposure, before and after dose increase

Group 1A: low exposure, PK-guided dose increase, group 1B: low exposure, no PK-guided dose increase due to toxicity, group 1C: low exposure, no toxicity, no PK-guided dose increase for other reasons.

Group 2: adequate exposure with (2B) or without (2A) toxicity.

$C_{\text {min }}=$ minimum plasma concentration; $P K=$ pharmacokinetic 


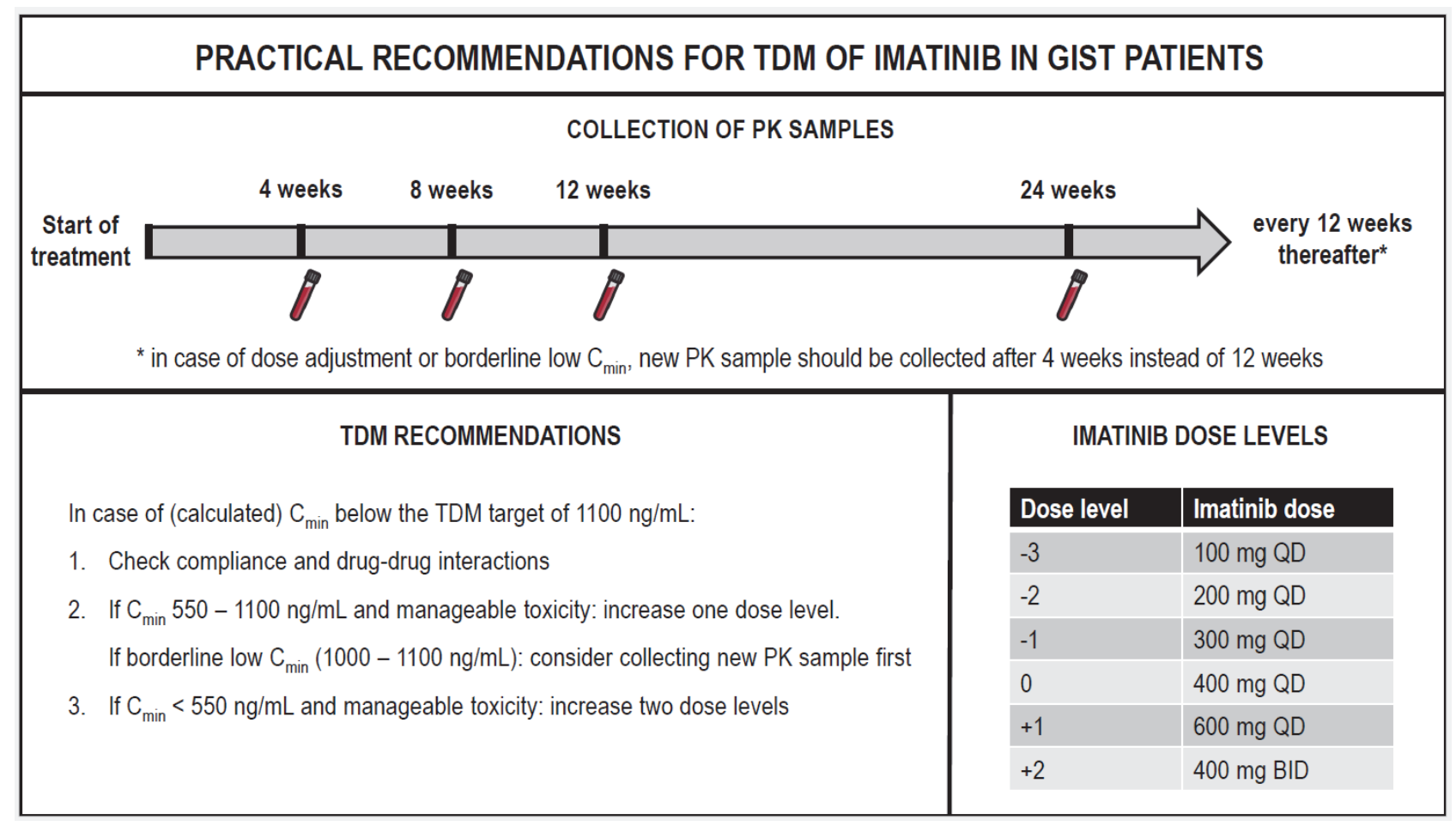

Figure 4 - Practical recommendations for TDM of imatinib in patients with GIST

$B I D=$ twice daily; $C_{\min }=$ minimum plasma concentration; $G I S T=$ gastrointestinal stromal tumour; $P K=$ pharmacokinetic; $Q D=$ once daily; TDM = therapeutic drug monitoring

\section{Treatment outcome}

The median follow-up time was 48 months (95\% confidence interval ( $\mathrm{Cl}$ ) 40.5-55.5). Sixtynine KIT exon 11 mutated patients received imatinib with palliative intent, of which 32 (46\%) had progressive disease. The median progression-free survival (mPFS) in these patients was 31 months (95\% Cl: 24.6-37.4) and did not differ between the groups (logrank test: $p=0.149$, Supplementary Figure 1). The mPFS was not reached for group $1 A$, was 30 months for group $1 \mathrm{~B}+1 \mathrm{C}(95 \% \mathrm{Cl}$ : 27.8-32.2) and 30 months for group $2 \mathrm{~A}+2 \mathrm{~B}(95 \%$ Cl: 4.8-55.3). Multivariable Cox regression yielded similar results (Supplementary Table 1).

\section{DISCUSSION}

In this manuscript, we described the largest cohort of patients with GIST in whom PKguided dose increases of imatinib were performed in daily clinical practice. In total, 75\% of patients had a low exposure. In $62 \%$ of these patients, a PK-guided dose increase was performed, which was successful in $63 \%$ of them. Patients with initial low PK who received PK-guided dose increases, levelled the outcome of patients with all adequate PK. Hence, TDM of imatinib is feasible in clinical practice and leads to an increased proportion of patients with adequate exposure. 
It is remarkable that in our cohort, patients with a low imatinib exposure appeared to be more often men. Pharmacologically this might be explained by a lower absorption or a higher clearance of imatinib in men than in women. In a population PK model, imatinib clearance was indeed found to be higher in men ${ }^{23}$, while other studies did not report an influence of gender on imatinib PK. ${ }^{6,9}$ In addition, PK-guided dose increases tended to be more often feasible in younger patients.

No differences in PFS were found between the three subgroups. Moreover, looking at the Kaplan-Meier curve (Supplementary Figure 1), it seems that group 2A+2B (adequate PK) has a similar PFS to $1 \mathrm{~B}+1 \mathrm{C}$ (low $\mathrm{PK}$, no intervention), while one would expect a longer PFS for group $2 \mathrm{~A}+2 \mathrm{~B}$ due to the known exposure-response relationship. ${ }^{2}$ However, these results are difficult to interpret for several reasons. First, subgroups were small and the number of events was still limited. Second, PFS in group 1B+1C (low PK, no intervention) was longer than expected from the literature (i.e. 30 months vs. 11.3 months ${ }^{2}$ ), possibly explained by the fact that exposure was only slightly below the target in this group (1050 $\mathrm{ng} / \mathrm{mL}$ ). Finally, patients who are longer on imatinib treatment have a higher chance of being classified as group 1 , as the chance of detecting a low $C_{\min }$ increases with an increasing number of PK samples per patient.

Theoretically, PK-guided dose increases could have been performed for patients in group $1 \mathrm{C}$ as well, as these patients had low PK without reported toxicity. In part of these patients, toxicity might still have played a role in the decision not to increase the dose but may not have been documented in the patient files. In addition, for some patients, $C_{\text {min }}$ was initially reported to be adequate, since at that time the described algorithm to estimate $C_{\min }$ was not used yet and a slightly lower threshold was used for reporting of the results $\left(C_{\min } \geq\right.$ $1000 \mathrm{ng} / \mathrm{mL}$, based on the target for $\left(\mathrm{ML}^{4,5}\right)$.

In a previous randomized controlled trial on TDM of imatinib between 2009-2011 by Gotta et al., dose recommendations were not followed up upon in almost half of the patients. ${ }^{24}$ In contrast, in our study, the lack of physician adherence was only encountered in nine patients (8\%). The higher physician adherence in our study compared with that of Gotta et al. may be explained by the passage of time and TDM becoming more accepted as routine clinical practice meanwhile.

The percentage of patients in whom a PK-guided dose increase is considered successful does depend on the applied definition of successful. In our opinion, a definition including tolerability and median exposure over time after the dose increase reflects the concept of successful. With this definition, the relatively high intra-individual variability of imatinib is taken into account. In addition, if treatment at a higher imatinib dose is only feasible for a short period of time due to toxicity, this should not be considered successful.

The majority of patients (75\%) had a low exposure according to the applied definition. This could raise the question whether the threshold of $1100 \mathrm{ng} / \mathrm{mL}$ is a valid target. Because 
imatinib has been demonstrated to be an effective treatment for GIST, it would be unlikely that the majority of patients are underdosed. In another exposure-response analysis, $C_{\text {min }}$ $\geq 760 \mathrm{ng} / \mathrm{mL}$ was identified as the optimal threshold. ${ }^{7}$ It has to be noted though, that this cohort seems not to be representative of the general GIST population, as PK samples were collected randomly instead of consecutively for all patients, and PFS was unexpectedly long compared with that of the literature. As the cohort of Demetri et al. is a better reflection of clinical practice, we decided to use their proposed threshold of $C_{\min } \geq 1100$ $\mathrm{ng} / \mathrm{mL}$ in our routine TDM.

Strengths of the current study include the accurate reflection of daily clinical practice and the measurement of multiple PK samples over time. In addition, to the best of our knowledge, this is the largest cohort of patients with GIST in whom PK-guided dose increases were performed in daily clinical practice. From our current cohort, 38\% of patients were also described in a previously published article on imatinib PK in patients with GIST. ${ }^{25}$ In that article, no PK-guided dose increases were described, as we do in the present article. Therefore, new to the present article is the focus on feasibility of TDM in daily clinical practice. Limitations could be that the reported $C_{\min }$ was not accompanied with a specific dose recommendation, which could have impaired the physician adherence. In addition, the methods used to estimate $C_{\min }$ are accompanied by some inherent limitations, for example, the formula does not correct for inter-individual variability in imatinib clearance. In addition, although data collection in the GIST registry is prospective, the current research questions were answered retrospectively, with the inherent limitations. Furthermore, the number of PK samples and treatment duration differ among patients, which could have influenced the results, and data on patient compliance are lacking. Finally, due to the small number of patients with metastatic disease, no definite conclusions on the effect on PFS could yet be drawn.

To conclude, these results emphasise the feasibility of TDM of imatinib in clinical practice. Although this has been routine clinical practice for patients with GIST in our hospital for several years now, this has not yet been applied worldwide. Efforts should be made to implement TDM as part of routine care for every patient. Real-life data of these patients should continuously be collected to further investigate the effects on treatment efficacy.

\section{ACKNOWLEDGEMENTS}

The authors would like to thank everyone who contributed to the logistics of collecting and measuring the imatinib concentrations, in particular Julie Janssen, Laura MolenaarKuijsten and René Boosman. Furthermore, the authors would like to thank all our colleagues that helped with the maintenance of the Dutch GIST Registry (DGR), for the Netherlands Cancer Institute (NKI), in particular Sheima Farag. 


\section{REFERENCES}

1. Güller U, Tarantino I, Cerny T, Schmied BM, Warschkow R. Population-based SEER trend analysis of overall and cancer-specific survival in 5138 patients with gastrointestinal stromal tumor. BMC Cancer 2015; 15: 557.

2. Demetri GD, Wang Y, Wehrle E, Racine A, Nikolova Z, Blanke CD et al. Imatinib plasma levels are correlated with clinical benefit in patients with unresectable/metastatic gastrointestinal stromal tumors. J Clin Oncol 2009; 27: 3141-3147.

3. Guilhot F, Hughes TP, Cortes J, Druker BJ, Baccarani M, Gathmann I et al. Plasma exposure of imatinib and its correlation with clinical response in the Tyrosine Kinase Inhibitor Optimization and Selectivity Trial. Haematologica 2012; 97: 731-738.

4. Larson RA, Druker BJ, Guilhot F, O'Brien SG, Riviere GJ, Krahnke $T$ et al. Imatinib pharmacokinetics and its correlation with response and safety in chronic-phase chronic myeloid leukemia: a subanalysis of the IRIS study. Blood 2008; 111: 4022-4028.

5. Picard S, Titier K, Etienne G, Teilhet E, Ducint D, Bernard MA et al. Trough imatinib plasma levels are associated with both cytogenetic and molecular responses to standard-dose imatinib in chronic myeloid leukemia. Blood 2007; 109: 3496-3499.

6. Yoo C, Ryu MH, Ryoo BY, Beck MY, Kang YK. Efficacy, safety, and pharmacokinetics of imatinib dose escalation to $800 \mathrm{mg} /$ day in patients with advanced gastrointestinal stromal tumors. Invest New Drugs 2013; 31: 1367-1374.

7. Bouchet S, Poulette S, Titier K, Moore N, Lassalle R, Abouelfath A et al. Relationship between imatinib trough concentration and outcomes in the treatment of advanced gastrointestinal stromal tumours in a real-life setting. Eur J Cancer 2016; 57: 31-38.

8. Eechoute K, Fransson MN, Reyners AK, de Jong FA, Sparreboom A, van der Graaf WT et al. A long-term prospective population pharmacokinetic study on imatinib plasma concentrations in GIST patients. Clin Cancer Res 2012; 18: 5780-5787.

9. Farag S, Verheijen RB, Martijn Kerst J, Cats A, Huitema AD, Steeghs N. Imatinib Pharmacokinetics in a Large Observational Cohort of Gastrointestinal Stromal Tumour Patients. Clin Pharmacokinet 2017; 56: 287-292.

10. Lankheet NAG, Desar IME, Mulder SF, Burger DM, Kweekel DM, van Herpen CML et al. Optimizing the dose in cancer patients treated with imatinib, sunitinib and pazopanib. $\mathrm{Br} J$ Clin Pharmacol 2017; 83: 2195-2204.

11. Lankheet NA, Knapen LM, Schellens JH, Beijnen JH, Steeghs N, Huitema AD. Plasma concentrations of tyrosine kinase inhibitors imatinib, erlotinib, and sunitinib in routine clinical outpatient cancer care. Ther Drug Monit 2014; 36: 326-334.

12. Zuidema S, Desar IME, van Erp NP, Kievit W. Optimizing the dose in patients treated with imatinib as first line treatment for gastrointestinal stromal tumors: A cost-effectiveness study. Br J Clin Pharmacol 2019; 85: 1994-2001.

13. Wang SY, Wu CE, Lai CC, Chen JS, Tsai CY, Cheng CT et al. Prospective Evaluation of Neoadjuvant Imatinib Use in Locally Advanced Gastrointestinal Stromal Tumors: Emphasis on the Optimal Duration of Neoadjuvant Imatinib Use, Safety, and Oncological Outcome. Cancers (Basel) 2019; 11: 424. 
14. Rutkowski $P$, Gronchi A, Hohenberger P, Bonvalot S, Schöffski P, Bauer S et al. Neoadjuvant imatinib in locally advanced gastrointestinal stromal tumors (GIST): the EORTC STBSG experience. Ann Surg Oncol 2013; 20: 2937-2943.

15. Joensuu $H$, Eriksson M, Sundby Hall K, Hartmann JT, Pink D, Schütte J et al. One vs three years of adjuvant imatinib for operable gastrointestinal stromal tumor: a randomized trial. JAMA 2012; 307: 1265-1272.

16. Casali PG, Abecassis N, Aro HT, Bauer S, Biagini R, Bielack S et al. Gastrointestinal stromal tumours: ESMO-EURACAN Clinical Practice Guidelines for diagnosis, treatment and followup. Ann Oncol 2018; 29: iv267.

17. Herbrink M, de Vries N, Rosing H, Huitema AD, Nuijen B, Schellens JH et al. Quantification of 11 Therapeutic Kinase Inhibitors in Human Plasma for Therapeutic Drug Monitoring Using Liquid Chromatography Coupled With Tandem Mass Spectrometry. Ther Drug Monit 2016; 38: 649-656.

18. Wang Y, Chia YL, Nedelman J, Schran H, Mahon FX, Molimard M. A therapeutic drug monitoring algorithm for refining the imatinib trough level obtained at different sampling times. Ther Drug Monit 2009; 31: 579-584.

19. Summary of Product Characteristics - Imatinib. Available from: https://www.ema.europa.eu/en/documents/product-information/glivec-epar-productinformation_en.pdf2001. Accessed 24 Feb 2020.

20. Janssen JM, Dorlo TPC, Beijnen JH, Huitema AD. Evaluation of extrapolation methods to predict trough concentrations to guide therapeutic drug monitoring of oral anticancer drugs. Ther Drug Monit 2020. Epub ahead of print.

21. Miettinen M, Lasota J. Gastrointestinal stromal tumors: pathology and prognosis at different sites. Semin Diagn Pathol 2006; 23: 70-83.

22. Casali PG, Zalcberg J, Le Cesne A, Reichardt P, Blay JY, Lindner LH et al. Ten-Year ProgressionFree and Overall Survival in Patients With Unresectable or Metastatic GI Stromal Tumors: Long-Term Analysis of the European Organisation for Research and Treatment of Cancer, Italian Sarcoma Group, and Australasian Gastrointestinal Trials Group Intergroup Phase III Randomized Trial on Imatinib at Two Dose Levels. J Clin Oncol 2017; 35: 1713-1720.

23. Widmer N, Decosterd LA, Csajka C, Leyvraz S, Duchosal MA, Rosselet A et al. Population pharmacokinetics of imatinib and the role of alpha-acid glycoprotein. Br J Clin Pharmacol 2006; 62: 97-112.

24. Gotta V, Widmer N, Decosterd LA, Chalandon Y, Heim D, Gregor M et al. Clinical usefulness of therapeutic concentration monitoring for imatinib dosage individualization: results from a randomized controlled trial. Cancer Chemother Pharmacol 2014; 74: 1307-19.

25. Farag S, Verheijen RB, Kerst MJ, Cats A, Huitema AD, Steeghs N. Imatinib Pharmacokinetics in a Large Observational Cohort of Gastrointestinal Stromal Tumour Patients. Clin Pharmacokinet 2017; 56: 287-292. 


\section{SUPPLEMENTARY DATA}

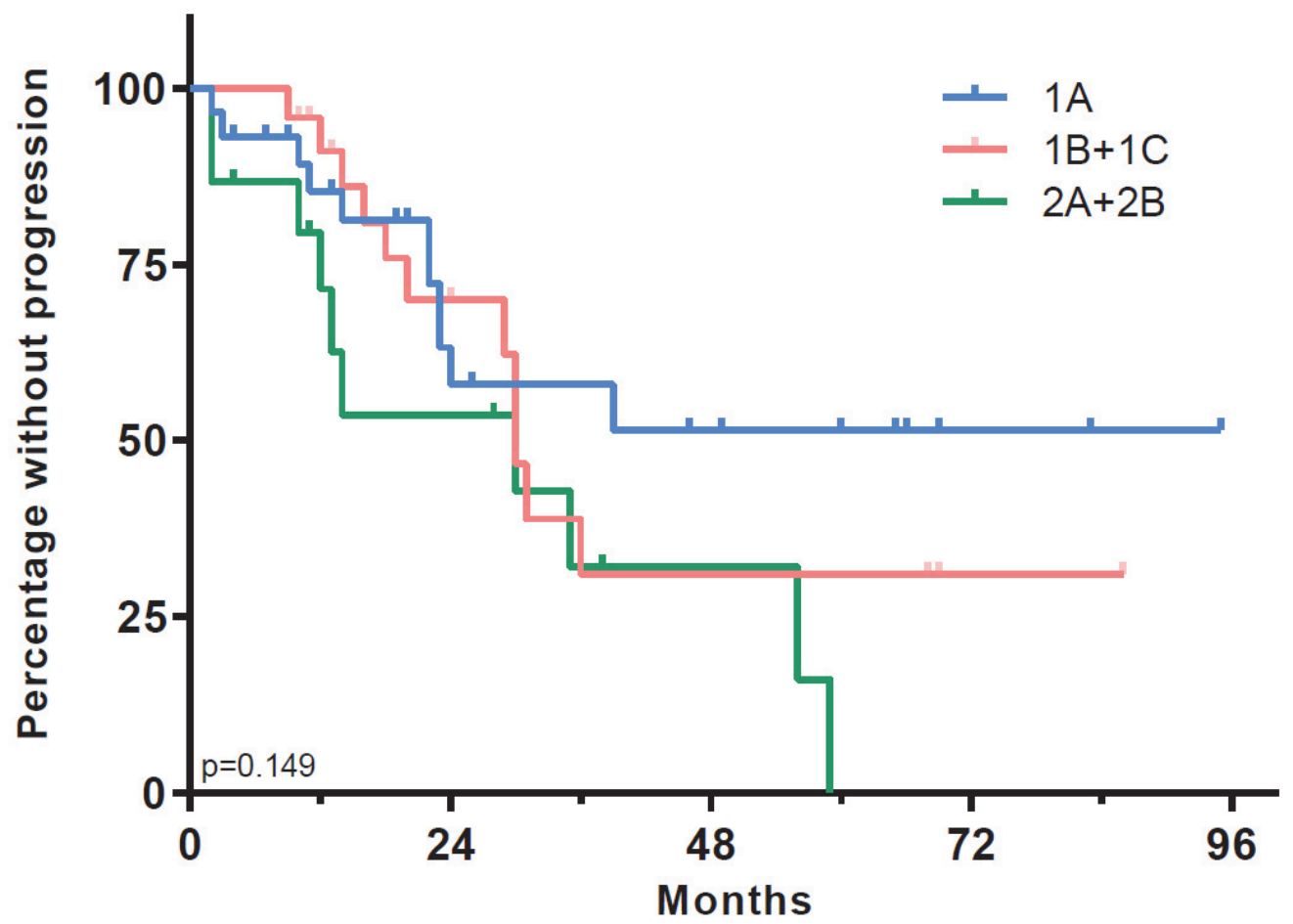

No. at risk $\mathrm{O} \quad \mathrm{N}$

$\begin{array}{lllllll}\text { 1A: } & 11 & 30 & 11 & 7 & 2 & 0 \\ \text { 1B+1C: } & 11 & 24 & 9 & 3 & 1 & 0 \\ 2 \mathrm{~A}+2 \mathrm{~B}: & 10 & 15 & 6 & 2 & 0 & 0\end{array}$

Supplementary Figure 1 - Progression-free survival of KIT exon 11 mutated patients in palliative treatment setting

Group 1A: low exposure, PK-guided dose increase, group 1B+1C: low exposure, no PK-guided dose increase, group 2A+2B: adequate exposure.

$O=$ number of observed events; $N=$ number of patients at risk 
Supplementary Table 1 - Cox regression analysis for progression-free survival in KIT exon 11 mutated patients treated in the palliative setting

\begin{tabular}{|c|c|c|c|c|c|c|}
\hline & \multicolumn{3}{|c|}{ Univariable analysis } & \multicolumn{3}{|c|}{ Multivariable analysis } \\
\hline & HR & $95 \% \mathrm{Cl}$ & p-value & HR & $95 \% \mathrm{Cl}$ & p-value \\
\hline \multicolumn{7}{|l|}{ Subgroup } \\
\hline $1 \mathrm{~A}$ & 0.439 & $0.186-1.040$ & 0.061 & 0.389 & $0.088-1.719$ & 0.213 \\
\hline $1 \mathrm{~B}+1 \mathrm{C}$ & 0.590 & $0.250-1.393$ & 0.228 & 0.517 & $0.143-1.868$ & 0.314 \\
\hline $2 A+2 B$ & Reference & & & & & \\
\hline Primary tumor size & 1.003 & $0.995-1.010$ & 0.485 & 1.008 & $0.999-1.017$ & 0.086 \\
\hline \multicolumn{7}{|l|}{$\begin{array}{l}\text { Primary GIST } \\
\text { location }\end{array}$} \\
\hline Gastric & Reference & & & & & \\
\hline Small bowel & 1.329 & $0.629-2.808$ & 0.456 & 1.689 & $0.573-4.978$ & 0.342 \\
\hline Other & 0.837 & $0.272-2.574$ & 0.757 & 0.968 & $0.229-4.095$ & 0.965 \\
\hline \multicolumn{7}{|l|}{$\begin{array}{l}\text { Baseline mitotic } \\
\text { rate }\end{array}$} \\
\hline Low & Reference & & & & & \\
\hline High & 1.688 & $0.699-4.077$ & 0.244 & 1.762 & $0.576-5.386$ & 0.321 \\
\hline Age at diagnosis & 1.040 & 1.009-1.071 & 0.010 & 1.027 & $0.982-1.074$ & 0.240 \\
\hline \multicolumn{7}{|l|}{ Gender } \\
\hline Male & Reference & & & & & \\
\hline Female & 1.095 & $0.505-2.372$ & 0.818 & 0.880 & $0.259-2.992$ & 0.838 \\
\hline \multicolumn{7}{|l|}{$\begin{array}{l}\text { Performance } \\
\text { status }\end{array}$} \\
\hline WHO 0 & Reference & & & & & \\
\hline $\mathrm{WHO} \geq 1$ & 0.749 & $0.337-1.666$ & 0.479 & 0.799 & $0.242-2.636$ & 0.713 \\
\hline
\end{tabular}

All selected univariable confounders were included in the multivariable model.

Group 1A: low exposure, PK-guided dose increase, Group 1B+1C: low exposure, no PK-guided dose increase, Group 2A+2B: adequate exposure all the time. 


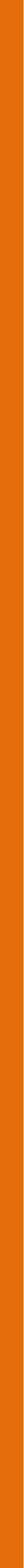




\section{ABSTRACT}

\section{Background}

Pazopanib is a tyrosine kinase inhibitor indicated for the treatment of renal cell carcinoma and soft tissue sarcoma. Despite the high inter-patient variability in pharmacokinetic exposure, pazopanib is administered at a fixed dose of $800 \mathrm{mg}$ once daily (QD). Pharmacokinetic exposure is linked to both efficacy and toxicity. In this case report, we illustrate the value of therapeutic drug monitoring by describing two patients with adequate pazopanib trough concentrations $\left(C_{\min }\right)$ at an eight times lower than standard dose.

\section{Case presentation}

Patient $A$ is a 69-year-old woman with metastatic leiomyosarcoma who had significant toxicities and a high $C_{\min }$ on the standard dose. While dose reductions to $200 \mathrm{mg}$ QD and later $200 \mathrm{mg}$ every other day were made, pazopanib $C_{\min }$ remained above the efficacy threshold. Patient B is a 50-year-old male with metastatic angiosarcoma and a history of Gilbert syndrome. Pazopanib treatment was initiated at the standard dose of $800 \mathrm{mg}$ QD, but was reduced to $200 \mathrm{mg}$ QD 1-week-on - 1-week-off due to total bilirubin elevation. Pazopanib $C_{\min }$ was adequate in this patient as well.

\section{Conclusion}

It could be valuable to measure pazopanib levels in case of dose reductions due to toxicity, as exposure could still be adequate at considerably lower than standard doses. 


\section{BACKGROUND}

Pazopanib is a tyrosine kinase inhibitor mainly targeting the vascular endothelial growth factor receptor and is indicated for the treatment of advanced renal cell carcinoma and soft tissue sarcoma. ${ }^{1}$ Despite the high inter-patient variability in exposure (40-70\%), pazopanib is administered at a fixed oral dose of $800 \mathrm{mg}$ once daily (QD). ${ }^{1,2}$ Suttle et al. reported a clear exposure-response relationship, with patients with a pazopanib trough concentration $\left(C_{\min }\right)$ above $20.5 \mathrm{mg} / \mathrm{L}$ having a significantly longer progression-free survival (PFS). Also, an exposure-toxicity relationship has been demonstrated, with an increasing incidence of toxicities such as hypertension, diarrhea, elevated alanine aminotransferase levels, stomatitis and hand-foot syndrome with increasing pazopanib plasma concentrations. ${ }^{3}$ It has been shown that patients are unlikely to tolerate $C_{\min } \geq 50$ $\mathrm{mg} / \mathrm{L}$ for a prolonged period of time. ${ }^{4}$ In this case report, we illustrate the value of therapeutic drug monitoring (TDM) for pazopanib by describing two patients with pazopanib $C_{\min }$ above the efficacy threshold of $20.5 \mathrm{mg} / \mathrm{L}$ at an eight times lower than standard dose.

\section{CASE PRESENTATION}

\section{Case A}

We present a 69-year-old woman with a history of metastatic leiomyosarcoma, for which pazopanib treatment was initiated at the standard dose of $800 \mathrm{mg}$ QD, after she progressed upon first-line chemotherapy with doxorubicin. During the first month of treatment pazopanib was temporarily withheld twice due to significant toxicities, including fatigue, nausea, vomiting and syncope. Pazopanib plasma concentrations were measured and $C_{\min }$ was calculated using the formula proposed by Wang et al. ${ }^{5}$, showing high pazopanib trough levels ( $36.1 \mathrm{mg} / \mathrm{L}$ and $41 \mathrm{mg} / \mathrm{L}$ ). Pazopanib treatment was resumed after sequential dose reductions to $600 \mathrm{mg}$ QD and $200 \mathrm{mg}$ QD. The last dose was well tolerated despite mild liver enzyme disorders and hypertension. During the following months, the patient developed diarrhea and hypothyroidism, after which pazopanib was further reduced to $200 \mathrm{mg}$ every other day. Pazopanib $C_{\min }$ remained adequate at this eight times lower than standard dose at first, although the last two measurements were below the efficacy threshold (Figure 1A). Unfortunately, 14 months after start of treatment, progressive disease was observed, after which chemotherapy with trabectedin was started.

\section{Case B}

The second case is a 50-year-old male with metastatic angiosarcoma and a history of Gilbert syndrome, previously treated with 6 cycles of doxorubicin in combination with ifosfamide. Pazopanib treatment was started at the standard dose of $800 \mathrm{mg}$ QD. Shortly 
hereafter, total bilirubin increased to twice the upper limit of normal with only minimal elevation of direct bilirubin, after which pazopanib was halted. Upon normalization of bilirubin, pazopanib treatment was resumed at a reduced dose of $400 \mathrm{mg}$ QD and later 200 mg QD 1-week-on - 1-week-off. At the end of the on-treatment week pazopanib $C_{\text {min }}$ was $29.9 \mathrm{mg} / \mathrm{L}$ (Figure 1B). The patient is still on treatment now, nine months after pazopanib initiation, with a partial remission.

\section{DISCUSSION AND CONCLUSION}

It is remarkable that even these unusually low doses of pazopanib lead to an adequate exposure, defined by $C_{\min } \geq 20.5 \mathrm{mg} / \mathrm{L}^{3}{ }^{3}$ In the absence of TDM, when doses would be reduced according to the Summary of Product Characteristics (SPC), treatment would probably have been discontinued in these patients, as the SPC considers the exposure at $200 \mathrm{mg}$ QD as markedly reduced and insufficient to obtain a clinically relevant effect. ${ }^{1}$ However, we observed an adequate $C_{\min }$ at considerably lower than standard doses and treatment could have been continued for 14 and 9 months (ongoing response), respectively. This is a relatively long duration of response taking into account the median PFS of 4.6 months for sarcoma patients found in the phase 3 trial. ${ }^{6}$ Although the efficacy threshold of $C_{\min } \geq 20.5 \mathrm{mg} / \mathrm{L}$ has been established in patients with renal cell carcinoma ${ }^{3}$, a similar trend has been demonstrated in sarcoma patients. ${ }^{7}$

Gilbert's syndrome, uridine diphosphoglucuronate glucuronosyltransferase 1A1 (UGT1A1) polymorphism, has been associated with pazopanib-induced hyperbilirubinemia, which was probably the case in the second patient. ${ }^{8}$ Given the benign etiology of this condition, continuing pazopanib treatment was possible, although in reduced dose.

With regard to obtaining the most adequate exposure, the dosing regimen of $200 \mathrm{mg}$ every other day is most rational, as with the $200 \mathrm{mg}$ 1-week-on - 1-week-off regimen patients will probably not have an adequate exposure in the off-treatment week. Unfortunately, we do not have any trough concentrations in the off-treatment week of patient B available.

As we illustrated with these two cases, it could be valuable to measure pazopanib levels in case of dose reductions due to toxicity, as $C_{\min }$ could still be above the efficacy threshold at considerably lower than standard doses. 
A

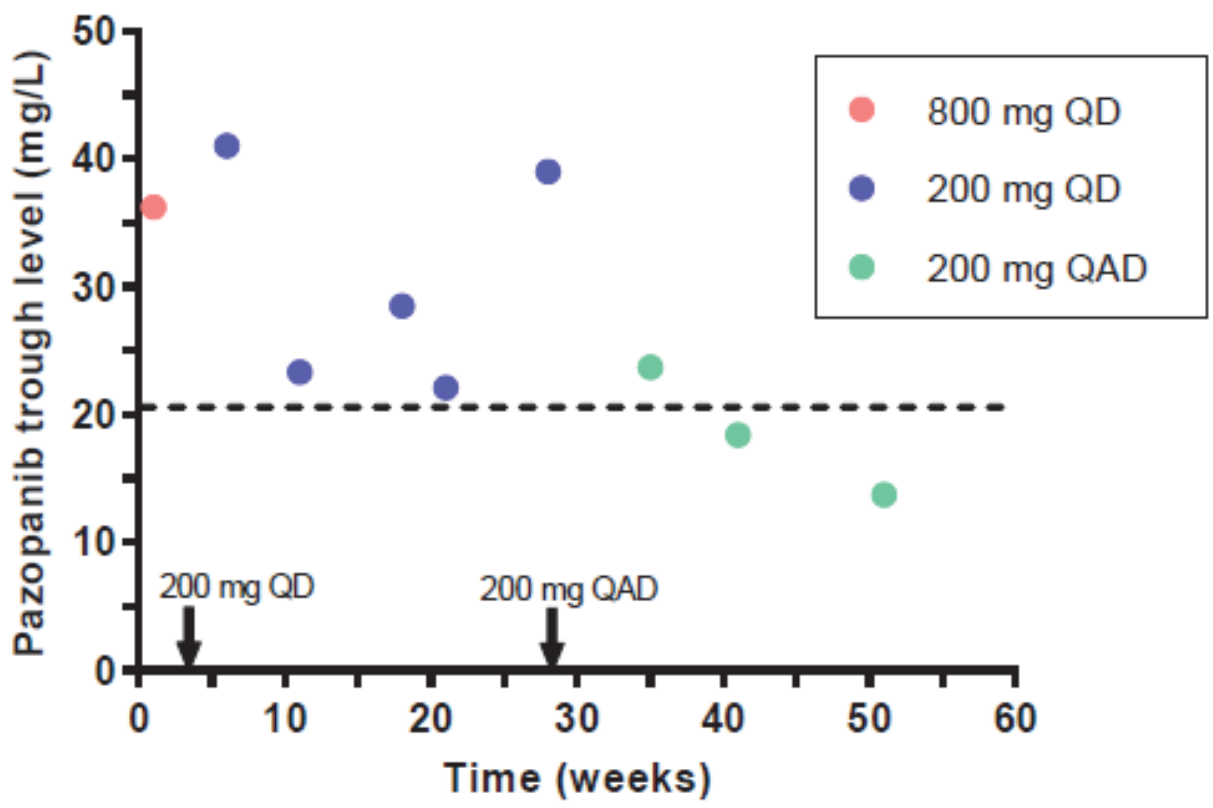

B

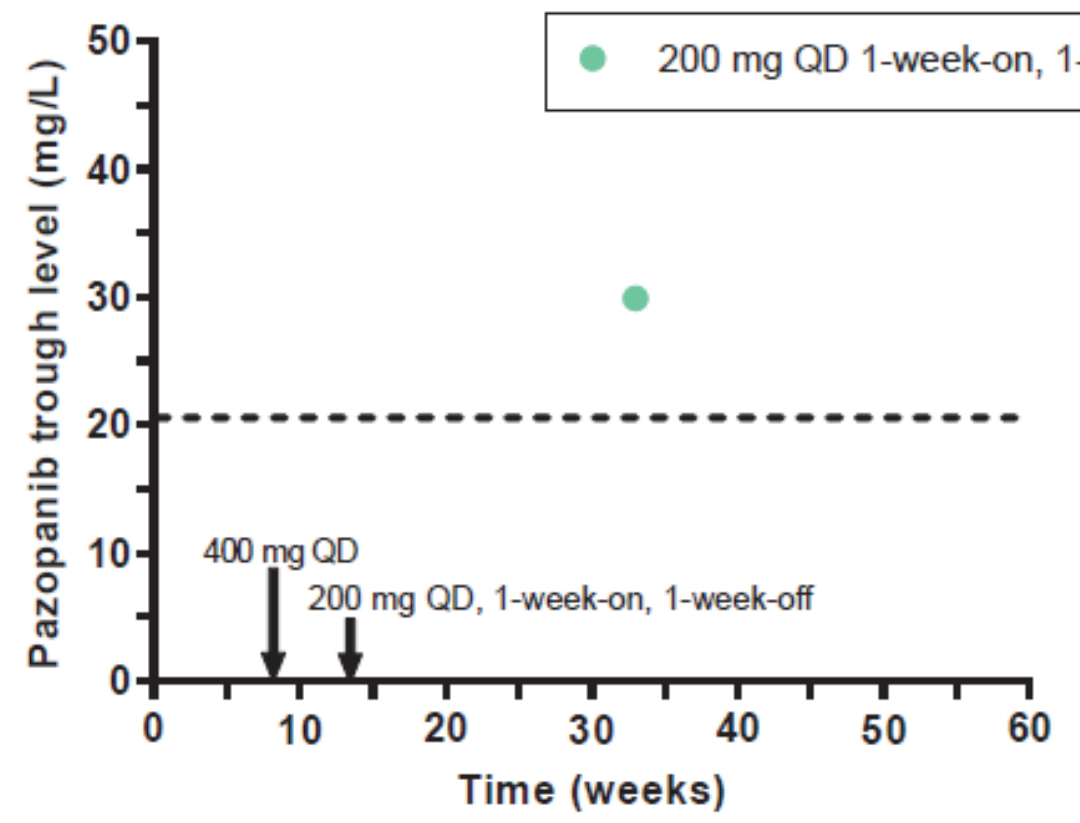

Figure 1 - Calculated pazopanib trough levels

A: For patient A, pazopanib dose was rapidly reduced to $200 \mathrm{mg}$ QD in the first three weeks and further reduced to $200 \mathrm{mg}$ every other day in week 28. Corresponding doses of pazopanib were: week 0-1 800 mg QD, week 1-2 stop, week 2-2.5 600 mg QD, week 2.5-3 stop, week 3-11.5 $200 \mathrm{mg}$ QD, week 11.5-13 stop, week 13-28 $200 \mathrm{mg}$ QD, week 28-59 $200 \mathrm{mg}$ every other day.

B: For patient B, pazopanib dose was reduced to $400 \mathrm{mg}$ QD after two months and to $200 \mathrm{mg}$ QD 1-week-on, 1-week-off after three months. Corresponding doses of pazopanib were: week 0-6 800 mg QD, week 6-8 stop, week 8-12 400 mg QD, week 12-13 stop, week 13-39 200 mg QD 1-weekon, 1-week-off.

The dashed line indicates the pharmacokinetic target of $C_{\min } \geq 20.5 \mathrm{mg} / \mathrm{L}$. The arrows indicate the time at which doses were changed.

$C_{\min }=$ minimum blood concentration; $Q A D=$ every other day; $Q D=$ once daily 


\section{REFERENCES}

1. Summary of Product Characteristics of pazopanib. Available from: http://www.ema.europa.eu/docs/nl_NL/document_library/EPAR_Product_Information/hu man/001141/WC500094272.pdf.

2. Hurwitz HI, Dowlati A, Saini S, Savage S, Suttle AB, Gibson DM et al. Phase I trial of pazopanib in patients with advanced cancer. Clin Cancer Res 2009; 15: 4220-4227.

3. Suttle AB, Ball HA, Molimard M, Hutson TE, Carpenter C, Rajagopalan D et al. Relationships between pazopanib exposure and clinical safety and efficacy in patients with advanced renal cell carcinoma. Br J Cancer 2014; 111: 1-8.

4. Verheijen RB, Bins S, Mathijssen RHJ, Lolkema MP, van Doorn L, Schellens JHM et al. Individualized pazopanib dosing: a prospective feasibility study in cancer patients. Clin Cancer Res 2016; 22: 5738-5746.

5. Wang Y, Chia Y, Nedelman J, Schran H, Mahon F, Molimard M. A therapeutic drug monitoring algorithm for refining the imatinib trough level obtained at different sampling times. Ther Drug Monit 2009; 31: 579-584.

6. Graaf WTA, Blay J-Y, Chawla SP, Kim D-W, Bui-Nguyen B, Casali PG et al. Pazopanib for metastatic soft-tissue sarcoma (PALETTE): a randomised, double-blind, placebo-controlled phase 3 trial. Lancet 2012; 379: 1879-1886.

7. Verheijen RB, Swart LE, Beijnen JH, Schellens JHM, Huitema ADR, Steeghs N. Exposuresurvival analyses of pazopanib in renal cell carcinoma and soft tissue sarcoma patients: opportunities for dose optimization. Cancer Chemother Pharmacol 2017; 80: 1171-1178.

8. $\mathrm{Xu} C-F$, Reck BH, Xue Z, Huang L, Baker $\mathrm{KL}$, Chen $\mathrm{M}$ et al. Pazopanib-induced hyperbilirubinemia is associated with Gilbert's syndrome UGT1A1 polymorphism. $\mathrm{Br} J$ Cancer 2010; 102: 1371-1377. 



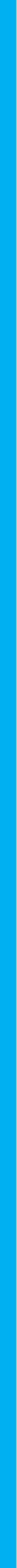




\section{ABSTRACT}

Oral anti-hormonal drugs are essential in the treatment of breast and prostate cancer. It is well known that the interpatient variability in pharmacokinetic exposure is high for these agents and exposure-response relationships exist for many oral anti-hormonal drugs. Yet, they are still administered at fixed doses. This could lead to under dosing and thus suboptimal efficacy in some patients, while other patients could be overdosed resulting in unnecessary side effects. Therapeutic drug monitoring (TDM), individualized dosing based on measured blood concentrations of the drug, could therefore be a valid option to further optimize treatment. In this review, we provide an overview of relevant clinical pharmacokinetic and pharmacodynamic characteristics of oral anti-hormonal drugs in oncology and translate these into practical guidelines for TDM.

For some agents, TDM targets are not well established yet and as a reference the median pharmacokinetic exposure could be targeted (exemestane: minimum plasma concentration $\left(C_{\min }\right) 4.1 \mathrm{ng} / \mathrm{mL}$ and enzalutamide: $\left.C_{\min } 11.4 \mathrm{mg} / \mathrm{L}\right)$. However, for most drugs, exposure-efficacy analyses could be translated into specific targets (abiraterone: $C_{\min } 8.4 \mathrm{ng} / \mathrm{mL}$, anastrozole: $C_{\min } 34.2 \mathrm{ng} / \mathrm{mL}$, and letrozole: $C_{\min } 85.6 \mathrm{ng} / \mathrm{mL}$ ). Moreover, prospective clinical trials have shown TDM to be feasible for tamoxifen, for which the exposure-efficacy threshold of its active metabolite endoxifen is $5.97 \mathrm{ng} / \mathrm{mL}$. Based on the available data, we therefore conclude that individualized dosing based on drug concentrations is feasible and promising for oral anti-hormonal drugs and should be developed further and implemented into clinical practice. 


\section{INTRODUCTION}

Breast and prostate cancer are both highly prevalent malignancies, with breast cancer being the most commonly diagnosed malignancy in women and prostate cancer in men in the Western world. Breast and prostate cancer represent the second leading cause of cancer deaths in women and men, respectively. ${ }^{1}$ As these tumors are often dependent on estrogens and androgens for their growth, anti-hormonal drugs are imperative in their treatment.

Even though many oral anti-hormonal drugs show exposure-response relationships and the interpatient variability in pharmacokinetic (PK) exposure is high (up to $141 \%$ for abiraterone $)^{2}$, they are still administered at fixed doses. As a result, some patients may be underdosed, which could lead to suboptimal efficacy, while other patients might be overdosed, causing unnecessary toxicity. Treatment could be optimized by therapeutic drug monitoring (TDM), which is individualized dosing based on measured blood concentrations of the drug. ${ }^{3-8}$

Use of TDM in oncology has been previously advocated, and for other targeted therapies, such as kinase inhibitors, TDM targets have been described previously. 3,4,6,7,9 The aim of this review is to summarize the available PK and pharmacodynamic (PD) data on oral antihormonal drugs, to discuss exposure-toxicity and exposure-efficacy relationships and to propose PK targets, which can be used for TDM.

Table 1 provides a summary of selected (steady state) PK parameters of these drugs. The proposed targets and TDM recommendations have been summarized in Table 2.

\section{METHODS}

Although this is not a systematic review, the literature was searched as comprehensively as possible. For all oral anti-hormonal drugs, the US FDA Clinical Pharmacology \& Biopharmaceutics Review and the Committee for Medicinal Products for Human Use European Public Assessment Report were consulted. Furthermore, PubMed searches were performed using the term "pharmacokinetics" in combination with the different oral anti-hormonal drugs. In addition, citation snowballing was used to find other relevant studies. 


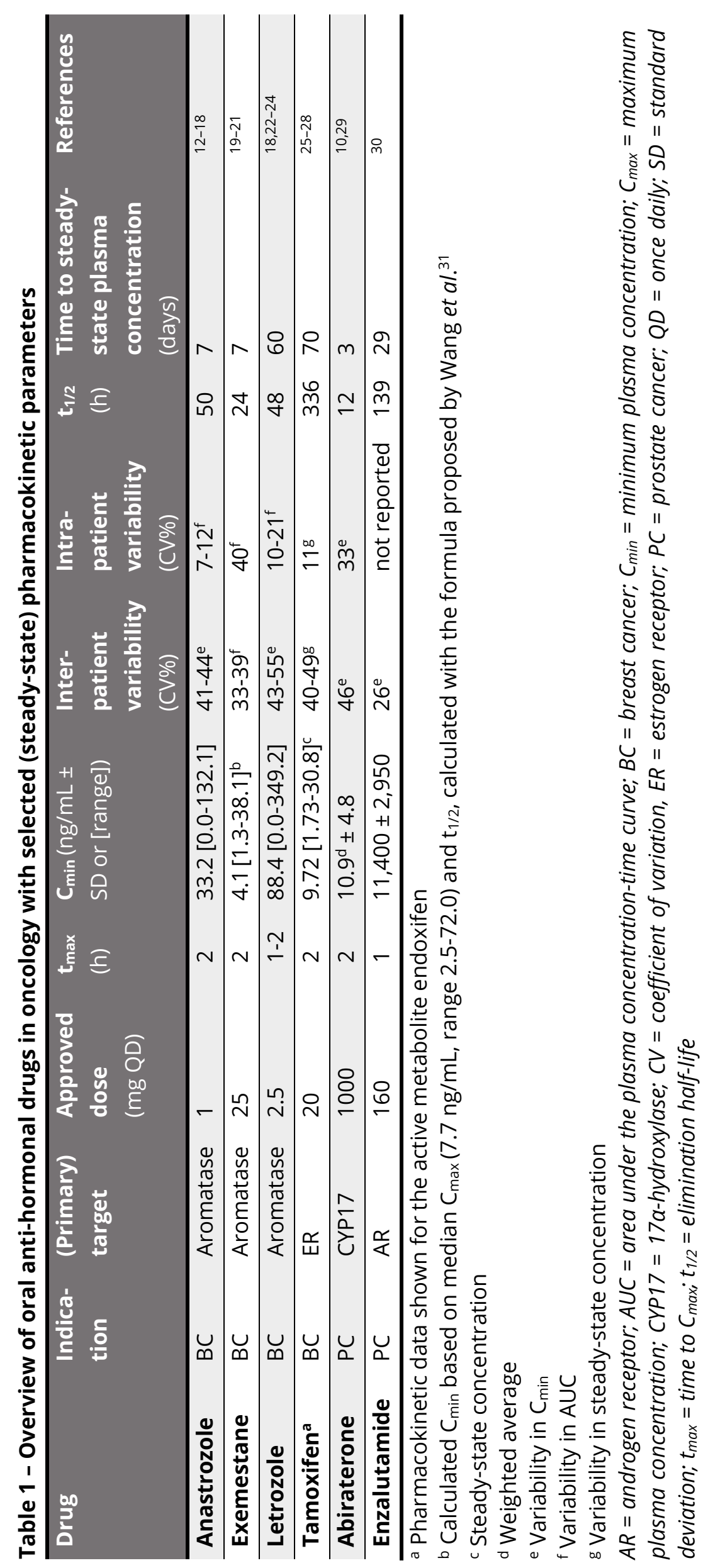


Table 2 - Overview of practical therapeutic drug monitoring (TDM) recommendations for oral anti-hormonal drugs in oncology

\begin{tabular}{|c|c|c|c|c|c|}
\hline Drug & $\begin{array}{l}\text { TDM } \\
\text { recommen- } \\
\text { dation }^{a}\end{array}$ & $\begin{array}{l}\text { Proposed } \\
\text { target } \\
(\mathrm{ng} / \mathrm{mL})\end{array}$ & $\begin{array}{l}\text { Mean/ } \\
\text { median } \\
\text { exposure } \\
\text { ( } C_{\text {min }} \text { in } \\
\mathrm{ng} / \mathrm{mL})\end{array}$ & $\begin{array}{l}\text { Outcome } \\
\text { parameter } \\
\text { associated } \\
\text { with TDM target }\end{array}$ & References \\
\hline Anastrozole & Exploratory & $C_{\min } \geq 34.2$ & 33.2 & $\begin{array}{l}\text { Estradiol } \\
\text { suppression }\end{array}$ & 12 \\
\hline Exemestane & Exploratory & & $4.1^{b}$ & & 20 \\
\hline Letrozole & Promising & $C_{\min } \geq 85.6$ & 88.4 & $\begin{array}{l}\text { Increased time to } \\
\text { tumor progression }\end{array}$ & 22 \\
\hline Tamoxifen & Viable & $C_{\min } \geq 5.97$ & 9.72 & $\begin{array}{l}\text { Lower recurrence } \\
\text { rate }\end{array}$ & 25,34 \\
\hline Abiraterone & Promising & $C_{\min } \geq 8.4$ & 10.9 & $\begin{array}{l}\text { PSA reduction, } \\
\text { progression-free } \\
\text { survival }\end{array}$ & 29,32 \\
\hline Enzalutamide & Exploratory & $C_{\min } \geq 5000$ & 11,400 & $\begin{array}{l}16 \beta-{ }^{18} \mathrm{~F}-5 a- \\
\text { dihydrotestosterone } \\
\text { imaging }\end{array}$ & 36 \\
\hline
\end{tabular}

\footnotetext{
a The provided TDM recommendation is considered promising if a pharmacokinetic TDM target is available, or viable if a prospective TDM study has been conducted. Otherwise, the recommendations are considered exploratory.

${ }^{b}$ Calculated $C_{\min }$ based on median $C_{\max }$ and $t_{1 / 2}$, calculated with the formula proposed by Wang et al. $^{31}$

$C_{\min }=$ minimum plasma concentration; $C_{\max }=$ maximum plasma concentration; $P S A=$ prostate-specific antigen; $t_{1 / 2}$ elimination half-life
}

\section{PRACTICAL RECOMMENDATIONS FOR THERAPEUTIC DRUG MONITORING OF ORAL ANTI-HORMONAL DRUGS IN ONCOLOGY}

\section{Anti-androgens}

\section{Abiraterone}

Abiraterone acetate (Zytiga $\left.{ }^{\circledR}\right)$ is the prodrug of abiraterone, which is a steroidal irreversible inhibitor of 17a-hydroxylase (cytochrome P450 (CYP) 17), thereby blocking the androgen synthesis. Abiraterone acetate is currently indicated for metastatic castrationresistant prostate cancer. ${ }^{10}$ In the near future, this indication might be expanded to patients with locally advanced or metastatic prostate cancer who are naive to antihormonal treatment. ${ }^{11}$

According to the Summary of Product Characteristics, abiraterone acetate should be ingested in modified fasting state, which means no food $2 \mathrm{~h}$ before or $1 \mathrm{~h}$ after drug 
intake. Chi et al. studied the food effect of the pharmacokinetics of abiraterone acetate and found a seven- and five-fold increase in maximum plasma concentration $\left(C_{\max }\right)$ and area under plasma concentration-time curve (AUC), respectively, with a low-fat meal and a 17- and 10-fold increase in $C_{\max }$ and AUC, respectively, with a high-fat meal, compared to overnight fasting in healthy subjects. However, in patients with metastatic castrationresistant prostate cancer, the food effect was compared to a modified fasting state, showing a less pronounced effect (similar exposure with low-fat meals and a twofold increase with high-fat meals). Adverse events (all grade $\leq 3$ ) were similar in the different groups (mainly hot flashes, fatigue, and hypokalemia). ${ }^{32}$

Abiraterone acetate has a high interpatient variability of $41-141 \%$ for AUC from time zero to infinity and $46 \%$ for $C_{\text {min }}$, with an intrapatient variability of $33 \% .2,29$ In the population-PK model described by Stuyckens et al. food and hepatic impairment appeared to be relevant covariates that influence abiraterone exposure, while $70 \%$ of the interpatient variability remained unexplained. ${ }^{33}$

Unfortunately, insufficient PK data have been collected in the pivotal trial and phase II trials to evaluate the exposure-toxicity relationships. ${ }^{10}$ Abiraterone acetate was generally well tolerated, and no dose-limiting toxicities were reported for doses up to $2000 \mathrm{mg}$ once daily (QD). ${ }^{37}$

A model has been developed in which a relationship between pharmacokinetics and prostate-specific antigen (PSA) reduction has been established. ${ }^{2,38}$ Additionally, in a prospective observational study in patients with castration-resistant prostate cancer $(n=61)$, higher abiraterone trough concentrations $\left(C_{\mathrm{min}}\right)$ were found in PSA responders compared to non-responders (12.0 vs. $8.0 \mathrm{ng} / \mathrm{mL}, \mathrm{p}=0.0015)$, in which PSA response was defined as a PSA decline of at least $50 \%$ after 3 months of treatment. ${ }^{29}$ The most predictive $C_{\text {min }}$ cut-off for PSA response was $8.4 \mathrm{ng} / \mathrm{mL}$ according to a receiver operating characteristic curve. Using this threshold, exposure-survival analysis found a progressionfree survival, defined as the time from treatment initiation to the first progression event (either PSA or radiologic progression), of 7.4 months in patients with a $C_{\text {min }}$ below 8.4 $\mathrm{ng} / \mathrm{mL}$ and 12.2 months in patients with a $C_{\min }$ above $8.4 \mathrm{ng} / \mathrm{mL}(\mathrm{p}=0.044)$. Nineteen of the 55 patients (35\%) in this study had a $\mathrm{C}_{\min }$ below $8.4 \mathrm{ng} / \mathrm{mL}^{29}$

Abiraterone is converted into the active metabolite $\Delta^{4}$-abiraterone (D4A) by the enzyme $3 \beta$-hydroxysteroid-dehydrogenase. Although the conversion ratio of abiraterone to D4A is low $( \pm 5 \%)$, it targets multiple steps of the androgen receptor signaling pathway, some of them more potently than abiraterone. An exposure-efficacy relationship has not been established for D4A hitherto, but given the dual mechanism of action of D4A (both inhibition of CYP17 and blockage of the androgen receptor), it is to be expected that such a relationship exists and may be identified. Therefore, measuring this metabolite could be interesting to refine TDM-guided dosing in the future..$^{39,40}$ 
Given the clear exposure-efficacy relationship, a target $C_{\min }$ of $\geq 8.4 \mathrm{ng} / \mathrm{mL}$ can be recommended for abiraterone. At the currently used fixed dose of $1000 \mathrm{mg}$ QD, 35\% of patients do not reach this threshold, with the potential to increase progression-free survival by 4.8 months for this subpopulation. Although it is advised to administer abiraterone in a modified fasting state, in clinical practice, patients often take it after an overnight fast. Since a clinically significant food effect has been shown when compared to overnight fasting, a first step in case of low exposure could be to administer abiraterone concomitantly with a light meal or a snack, before escalating the dose.

\section{Enzalutamide}

Enzalutamide (Xtandi ${ }^{\circledR}$ ) is an androgen-receptor antagonist indicated for the treatment of metastatic castration-resistant prostate cancer. ${ }^{30}$ Enzalutamide is metabolized by CYP2C8 and CYP3A4/5 to an inactive carboxylic acid (M1) and an active N-desmethyl metabolite (M2). The mean \pm standard deviation $C_{\min }$ of the approved 160-mg QD dose was $11.4 \pm 3.0 \mathrm{mg} / \mathrm{L}$ for enzalutamide, $13.0 \pm 3.8 \mathrm{mg} / \mathrm{L}$ for $\mathrm{M} 2$, and $8.4 \pm 6.8 \mathrm{mg} / \mathrm{L}$ for $\mathrm{M} 1{ }^{4}{ }^{4}$ Since $\mathrm{M} 2$ has a high abundancy and similar potency to enzalutamide, and concentrations of these two compounds can differ between patients $( \pm 25 \%)$, it could be scientifically interesting to measure both enzalutamide and its M2 metabolite. ${ }^{30}$ Future studies should clarify the role of this M2 metabolite in TDM.

No clinically significant exposure-toxicity relationships have been found so far. ${ }^{30}$

An exposure-efficacy analysis has been executed for enzalutamide in the pivotal phase III study, using the sum of the $C_{\min }$ for enzalutamide and $M 2$ with overall survival as the endpoint. All quartiles performed significantly better than placebo $(p<0.0001)$, yet no differences between the exposure quartiles could be found $(p \geq 0.5499) .{ }^{41}$

In the phase I/II trial, PSA decreases at 12 weeks were comparable in the different dose levels (range: 60-600 mg). ${ }^{36}$ Although enzalutamide targets the androgen receptor and could therefore cause a PSA decline without reflecting tumor response, it has been shown that PSA decline after 12 weeks is still associated with progression-free survival and overall survival. ${ }^{42}$

In the phase I trial, $16 \beta-{ }^{18} \mathrm{~F}-5 \mathrm{a}$-dihydrotestosterone positron emission tomography imaging in 22 patients suggested androgen receptor binding was higher in the 150-mg (corresponding to a median $C_{\min }$ of $11.4 \mathrm{mg} / \mathrm{L}$ ) than in the $60-\mathrm{mg}$ dose group (corresponding to a median $C_{\min }$ of approximately $5 \mathrm{mg} / \mathrm{L}$ ). No additional effect was seen at higher doses, suggesting a plateau at a dose of $150 \mathrm{mg}$ and $C_{\min }$ of $12 \mathrm{mg} / \mathrm{L}^{36}$

Given the lack of an exposure-toxicity relationship, the limited evidence for an exposureefficacy relationship, and the small interpatient variability in exposure ( $26 \%$ for $C_{\min }$ ), enzalutamide may not be the ideal drug for TDM. In absence of an exposure-efficacy target, the mean $C_{\min }$ of $11.4 \mathrm{mg} / \mathrm{L}$ at the standard dose of $160 \mathrm{mg}$ QD could be used as a 
reference. As this is the mean exposure, approximately $50 \%$ of patients will have concentrations below this reference. Based on the $16 \beta-{ }^{18} \mathrm{~F}-5 \mathrm{a}$-dihydrotestosterone data, dose increments could be considered in patients with a very low (e.g. $<5 \mathrm{mg} / \mathrm{L}$ ) $\mathrm{C}_{\mathrm{min}}$. Taking into account the mean exposure and standard deviation, less than $2.5 \%$ of patients will have trough concentrations $<5 \mathrm{mg} / \mathrm{L}$.

\section{Anti-estrogens}

\section{Tamoxifen}

Tamoxifen (Nolvadex $®$ ) is an estrogen receptor antagonist indicated for the treatment of estrogen receptor-positive breast cancer. Tamoxifen is extensively metabolized mainly by CYP2D6 and CYP3A4 into a range of active and inactive metabolites. ${ }^{43}$ Endoxifen is one of the most potent and abundant metabolites and, therefore, TDM of tamoxifen has focused on measuring endoxifen concentrations. Endoxifen shows a large interpatient variability in steady-state concentrations of $40-49 \%$, while the intrapatient variability is only $11 \% .^{25,27,28}$

No clear relationship between endoxifen concentration and toxicity has been reported in the literature. A retrospective study $(n=109)$ could not find evidence for an association between exposure and hot flashes, a major side effect of tamoxifen treatment. ${ }^{44}$ In another prospective trial ( $n=122)$, no significant correlation was found between tamoxifen metabolites and hot flash score $(p=0.07){ }^{45}$

A retrospective analysis of 1370 patients with estrogen receptor-positive breast cancer receiving tamoxifen in the adjuvant setting, found that patients in the lowest endoxifen exposure quintile (0-5.9 ng/mL) had a higher risk of recurrence than patients above this threshold (hazard ratio $0.74 ; 95 \%$ confidence interval 0.55-1.00). The recurrence rate was $16 \%$ for patients in the lowest quintile vs. $10.1-14.7 \%$ in the higher exposure quintiles. The investigators also explored dichotomized optimal cut-off points for the association between endoxifen concentrations and additional breast cancer events, in which an endoxifen concentration $\geq 5.97 \mathrm{ng} / \mathrm{mL}$ was the best threshold. This threshold corresponds closely to the lowest quintile. ${ }^{34}$

With the same dataset, an anti-estrogenic activity score was developed taking into account the $\mathrm{IC}_{50}$-corrected concentrations of tamoxifen, endoxifen, 4-hydroxytamoxifen, and $\mathrm{N}$ desmethyltamoxifen. ${ }^{46}$ An anti-estrogenic activity score threshold of 1798 was associated with a hazard ratio of 0.69 (95\% confidence interval $0.48-0.99$ ). It should be noted that this anti-estrogenic activity score was dominated by endoxifen, suggesting that endoxifen can serve as a proxy for the overall anti-estrogenic effect of tamoxifen and its metabolites.

While a clear exposure-efficacy relationship has been demonstrated in the adjuvant setting, Neven et al. did not find this relationship in the neo-adjuvant and metastatic setting. ${ }^{47}$ 
In a recent prospective clinical trial $(n=122)$, tamoxifen doses were tailored based on endoxifen concentrations. ${ }^{45}$ Breast cancer patients with an endoxifen concentration $<5.6$ $\mathrm{ng} / \mathrm{mL}$ (corresponding to $15 \mathrm{nmol} / \mathrm{L}$ ) received a 20-mg dose increase, while patients with endoxifen concentrations between 5.6 and $11.2 \mathrm{ng} / \mathrm{mL}$ (or 15-30 nmol/L) were recommended a dose increase of $10 \mathrm{mg}$. All patients with endoxifen concentrations $\geq 11.2$ $\mathrm{ng} / \mathrm{mL}$ continued treatment at the fixed dose of $20 \mathrm{mg}$ of tamoxifen. In total, 68 of 122 patients had at least one dose increment, after which $96 \%$ of patients achieved an endoxifen concentration $\geq 5.6 \mathrm{ng} / \mathrm{mL}$, compared to only $76 \%$ at baseline. ${ }^{45}$

Although it is known that CYP2D6 intermediate and poor metabolizer phenotypes are associated with lower endoxifen concentrations, the CYP2D6 phenotype only accounts for $18-43 \%$ of the interpatient variability in endoxifen concentrations. ${ }^{34,47-49}$ Twenty-four percent of the poor metabolizers and $58 \%$ of the intermediate metabolizers still have an endoxifen concentration above the efficacy threshold, while $12 \%$ of the normal metabolizers do not reach this threshold ${ }^{34}$ As endoxifen concentrations cannot be adequately predicted by the CYP2D6 phenotype, we advocate endoxifen-guided dosing instead of genotype-guided dosing.

At the currently used fixed dose of $20 \mathrm{mg}$ QD, $20 \%$ of patients do not reach the proposed efficacy threshold of $5.97 \mathrm{ng} / \mathrm{mL}$, with the potential to lower the recurrence rate by $26 \%$ in this subpopulation. The presence of a large retrospective exposure-efficacy study and prospective dose individualization study support the conclusion that it is feasible to dose tamoxifen based on measured endoxifen concentrations, using $\geq 5.97 \mathrm{ng} / \mathrm{mL}$ as a threshold, although no unequivocal evidence from a prospective trial is available yet, which demonstrates that TDM increases tamoxifen treatment efficacy.

\section{Aromatase inhibitors}

Estrogens are synthesized from androgens by the aromatase enzyme complex. This enzyme system is inhibited by aromatase inhibitors (Als). After previous use of the firstand second-generation Als (e.g. aminoglutethimide and formestane), the third-generation Als currently used in clinical practice are anastrozole, letrozole, and exemestane. These drugs are indicated for the treatment of postmenopausal patients with estrogen receptorpositive breast cancer, either in the (neo)adjuvant or metastatic setting. ${ }^{13,19,22}$ Anastrozole and letrozole are non-steroidal Als that bind reversibly to aromatase while exemestane is a steroidal Al that binds irreversibly to aromatase. ${ }^{50}$

Since Als inhibit the synthesis of estrogens, measuring circulating estrogen levels would be a good biomarker for efficacy. However, the sensitivity of the currently most commonly used estrogen assays is insufficient to measure the low concentrations of circulating estradiol in postmenopausal women, especially in those receiving Al treatment. ${ }^{51,52}$ Patients receiving anastrozole, letrozole, and exemestane treatment have median estradiol concentrations of $1.26,0.63$, and $0.63 \mathrm{pg} / \mathrm{mL}$, respectively. ${ }^{53,54}$ In daily clinical 
practice, circulating estradiol is measured using immunoassays (optimized to measure concentrations between $40-2000 \mathrm{pg} / \mathrm{mL}^{52}$ ), while mass spectrometry would be a more sensitive method, although this method is more costly and labor intensive. Even mass spectrometry assays are not always sensitive enough to measure the low circulating concentrations of estradiol in patients receiving Al treatment, for which assays with a lower limit of quantification (LLOQ) of 0.1-0.2 pg/mL are needed..$^{52} \mathrm{~A}$ recently published article suggested measuring gonadotrophins as a possible surrogate marker for estrogen activity. ${ }^{55}$ Future studies are needed to confirm the feasibility of gonadotrophins as a biomarker for efficacy of Als.

Hypothetically, one could imagine the dosing of Als could be personalized using a pharmacodynamic biomarker, such as measured estradiol concentrations or gonadotrophin levels. In absence of these data, individualized dosing based on pharmacokinetics is more within reach.

\section{Anastrozole}

Ingle et al. reported a high variability in anastrozole concentrations at the standard dose of $1 \mathrm{mg} \mathrm{QD}$, with a median of $33.2 \mathrm{ng} / \mathrm{mL}$, interquartile range $23.5-44.8 \mathrm{ng} / \mathrm{mL}$, and a range from LLOQ $(0.1 \mathrm{ng} / \mathrm{mL})$ to $132.1 \mathrm{ng} / \mathrm{mL}(\mathrm{n}=649)^{12}$, while the intra-patient variability is small $(7-12 \%) .^{18}$

To our knowledge, no exposure-toxicity relationship has been described for anastrozole. In phase I studies patients received repeated doses up to $10 \mathrm{mg}$ QD and single doses up to $60 \mathrm{mg}$ QD, which were well tolerated and did not cause any serious adverse events. ${ }^{14}$ A linear dose-exposure relationship was found for doses of 0.5 up to $10 \mathrm{mg} .{ }^{14}$

Dose-efficacy and exposure-efficacy relationships have only been studied with estrogen suppression as a surrogate marker of effect. Although previous studies showed estradiol suppression to below the limits of detection (LLOQ $2 \mathrm{pg} / \mathrm{mL}$ ) for doses of $1 \mathrm{mg}$ or higher ${ }^{14,56}$, it could still be possible that higher doses suppress estradiol to a greater extend, which could not have been quantified with these assays.

In a prospective study ( $n=649)$, Ingle et al. reported significantly lower anastrozole concentrations in patients with stable or increased estradiol concentrations compared with patients with decreased estradiol concentrations (LLOQ $0.625 \mathrm{pg} / \mathrm{mL}$ ) after the start of anastrozole treatment ( 26.7 vs. $34.2 \mathrm{ng} / \mathrm{mL}, \mathrm{p}<0.001) .{ }^{12}$ This indicates that TDM could be of value for anastrozole. However, because not all patients with decreased estradiol concentrations compared to baseline necessarily have sufficient estrogen suppression, higher anastrozole concentrations might be needed to attain adequate estrogen suppression.

No definitive exposure-efficacy target has been proposed yet for anastrozole. However, based on the available data, dose escalation could be considered for patients with a $C_{\text {min }}$ 
$<34.2 \mathrm{ng} / \mathrm{mL}^{12}$ Since the median exposure is $33.2 \mathrm{ng} / \mathrm{mL}$, approximately $50 \%$ of patients will have a $C_{\min }$ below this threshold at the currently used fixed dose of $1 \mathrm{mg}$ QD. Future studies should further investigate the relationship of anastrozole plasma concentrations with both circulating estrogen levels and progression-free and recurrence-free survival.

\section{Exemestane}

Although exemestane is extensively metabolized, 17-hydroxy-exemestane is the only active metabolite. However, because the 17-hydroxy-exemestane concentration is ten times lower than the exemestane concentration and 17-hydroxy-exemestane is 2.6 times less potent than exemestane, its additional anti-estrogenic effect is limited. ${ }^{19}$

Estradiol and exemestane share the same steroidal backbone. This structural resemblance can lead to falsely elevated estradiol concentrations in immunoassays. Therefore, measuring estradiol concentrations with liquid chromatography-tandem mass spectrometry instead of immunoassays therefore is the preferred option. ${ }^{57}$

Hertz et al. reported a median $\mathrm{C}_{\max }$ of exemestane of $7.7 \mathrm{ng} / \mathrm{mL}$ (range: $2.5-72.0, \mathrm{n}=246$ ) at the standard daily dose of $25 \mathrm{mg}$. Higher exemestane concentrations have been associated with the CYP3A4*22 variant, white race, elevated liver enzymes, renal insufficiency, lower body mass index (BMI), and not having received prior chemotherapy (all $p<0.05$ ). However, these factors explained less than $10 \%$ of the overall interpatient variability in exemestane concentrations. ${ }^{20}$

No exposure-toxicity relationship has been shown for exemestane. In general, exemestane is well tolerated, with single doses up to $800 \mathrm{mg}$ and multiple doses up to $200 \mathrm{mg}$ administered in phase I studies. ${ }^{19}$

Exposure increases proportionally with increasing dose. Estrogen suppression was maximal at a dose of $25 \mathrm{mg}$ (used assay is not mentioned). ${ }^{19}$ However, exemestane concentrations were not significantly different in patients who did and did not achieve estradiol suppression to undetectable concentrations (LLOQ $1.25 \mathrm{pg} / \mathrm{mL}$ ). ${ }^{58}$

No exposure-efficacy analyses have been reported yet for exemestane. Future studies need to explore any relationship between exemestane exposure and clinical response. In the absence of an exposure-efficacy target, the median $C_{\max }$ of $7.7 \mathrm{ng} / \mathrm{mL}$ could be used as a reference for TDM, corresponding to a calculated trough concentration of 4.1 $\mathrm{ng} / \mathrm{mL} .{ }^{31}$ As this is the median exposure, approximately $50 \%$ of patients will have trough concentrations below this proposed reference.

\section{Letrozole}

Desta et al. reported a high interpatient variability, with a median steady-state exposure of $88.4 \mathrm{ng} / \mathrm{mL}$ (range: LLOQ (7 ng/mL)-349.2 ng/mL) at the standard dose of $2.5 \mathrm{mg}$ QD. ${ }^{23}$ Higher exposure was associated with increasing age, lower BMI, and CYP2A6 genetic variations. The lower exposure with increasing BMI can be explained by the fact that 
letrozole is a highly lipophilic drug with a large volume of distribution (183L), which increases with increasing BMI. These three variables explain only $32.3 \%$ of the interpatient variability, thus a large proportion remains to be elucidated. ${ }^{23}$

In phase I studies, single doses up to $30 \mathrm{mg}$ and repeated doses up to $5 \mathrm{mg}$ were well tolerated. Higher exposure did not cause increased toxicity. ${ }^{22}$ Exposure increases approximately linearly with doses up to the standard dose of $2.5 \mathrm{mg} \mathrm{QD}$, while at higher doses the exposure increases non-linearly. ${ }^{22}$

No significant relationship was found between dose (range 0.5-5.0 mg) and estrogen suppression, albeit the assay used may not have been sensitive enough (LLOQ 2.5 $\mathrm{pg} / \mathrm{mL}){ }^{22}$ Furthermore, Hertz et al. found that median steady-state concentrations of letrozole were comparable in patients who did and did not achieve E2 suppression to undetectable concentrations (LLOQ $1.25 \mathrm{pg} / \mathrm{mL}, 88.8$ vs. $105.7 \mathrm{ng} / \mathrm{mL}$, respectively, $\mathrm{p}=0.63) .{ }^{58}$

In an exposure-efficacy analysis, patients were divided into groups reaching different letrozole plasma concentrations. This analysis showed a tendency to an increase in the time to tumor progression for those patients with a letrozole plasma concentration $\geq 85.6$ $\mathrm{ng} / \mathrm{mL} .{ }^{22}$ Future studies need to confirm this exposure-efficacy relationship. Until then, the most appropriate target for TDM of letrozole is $85.6 \mathrm{ng} / \mathrm{mL}$. Because the median exposure is $88.4 \mathrm{ng} / \mathrm{mL}$, slightly less than $50 \%$ of patients will not reach this target at the currently used fixed dose of $2.5 \mathrm{mg}$ QD.

\section{DISCUSSION}

The data presented in this review highlight clear opportunities to improve and to optimize treatment with anti-hormonal agents in oncology through TDM. However, the evidence for this is not equally strong for all agents. Because of this, we evaluated the available evidence and proposed TDM recommendations, which we considered either exploratory, promising, or viable, as presented in Table 2. The provided TDM recommendation is considered promising if a PK TDM target is available or viable if a prospective TDM study has been conducted. Otherwise the recommendations are considered exploratory.

Future studies are needed to explore exposure-efficacy relationships for those oral antihormonal drugs that are classified as exploratory (anastrozole, enzalutamide, and exemestane). In addition, prospective clinical studies should be performed to demonstrate the safety and feasibility of TDM for those oral anti-hormonal drugs that are classified as promising (abiraterone and letrozole). Ideally, for those drugs for which TDM is viable (tamoxifen), randomized controlled trials comparing TDM and fixed dosing with regard to relevant clinical efficacy endpoints such as progression-free survival and overall survival would be needed. Then, TDM could be fully integrated in clinical practice and become the standard of care. However, given the large patient numbers needed to 
conduct adequately powered randomized controlled trials, especially in the adjuvant setting, this will be a major challenge. Instead, future research could focus on prospective clinical studies strengthening the evidence of the PK target and confirming the safety and feasibility of TDM. ${ }^{59}$

Currently, exposure-efficacy and exposure-toxicity analyses are pivotal parts of the drug development process. ${ }^{60}$ However, in the era in which most of the older oral anti-hormonal drugs were registered, this was uncommon, resulting in a paucity of PK-PD data for these agents. Nonetheless, a PK target could be identified for four of the seven included agents. Overall, these targets amounted to $85 \%$ ( $\pm 19 \%$ ) of the mean population exposure (Figure 1). This is in accordance with the data for kinase inhibitors, as reported previously of $82 \%$ $( \pm 17 \%) .{ }^{3,4}$ Thus, targeting the mean exposure, in the absence of exposure-efficacy analyses, generally leads to adequate concentrations.

While awaiting a TDM target based on exposure-response analyses, measuring drug concentrations and collecting data on the efficacy and toxicity in routine patient care can provide us with valuable data on exposure-efficacy and exposure-toxicity relationships, comparable to safety monitoring as part of post-marketing surveillance.

To measure drug concentrations of oral anti-hormonal drugs, validated bio-analytical assays are needed. Our methods for the quantification of abiraterone, enzalutamide, and endoxifen have been previously published. ${ }^{61-63}$ Additionally, methods on the quantification of anastrozole, letrozole, and exemestane have been published by other investigators. ${ }^{64-66}$ Currently, we are validating an assay for the simultaneous measurement of all mentioned oral anti-hormonal drugs, which makes this a suitable assay for TDM in clinical practice.

Apart from the apparent advantages of TDM, another potential advantage for antihormonal drugs could be the monitoring of medication adherence, as it is has been shown that compliance decreases with long-term treatment. ${ }^{67}$ Additionally, TDM could help in detecting drug-drug interactions.

Since many of the anti-hormonal drugs have considerably long elimination half-lives, PK sampling for TDM should be timed appropriately to ensure that steady-state concentration has been achieved. In Table 1, the time until steady-state concentration has been reached is specified for the different compounds.

In disciplines other than oncology, TDM is being broadly applied, for example in patients using antibiotics, antiretroviral drugs, and immunosuppressants. An important difference, however, is the fact that in oncology we are reluctant to reduce the dose in the case of high drug concentrations because tumor progression is irreversible. For this reason, we advise to increase the dose in the case of low drug concentrations, while reducing doses only in the case of toxicity. 


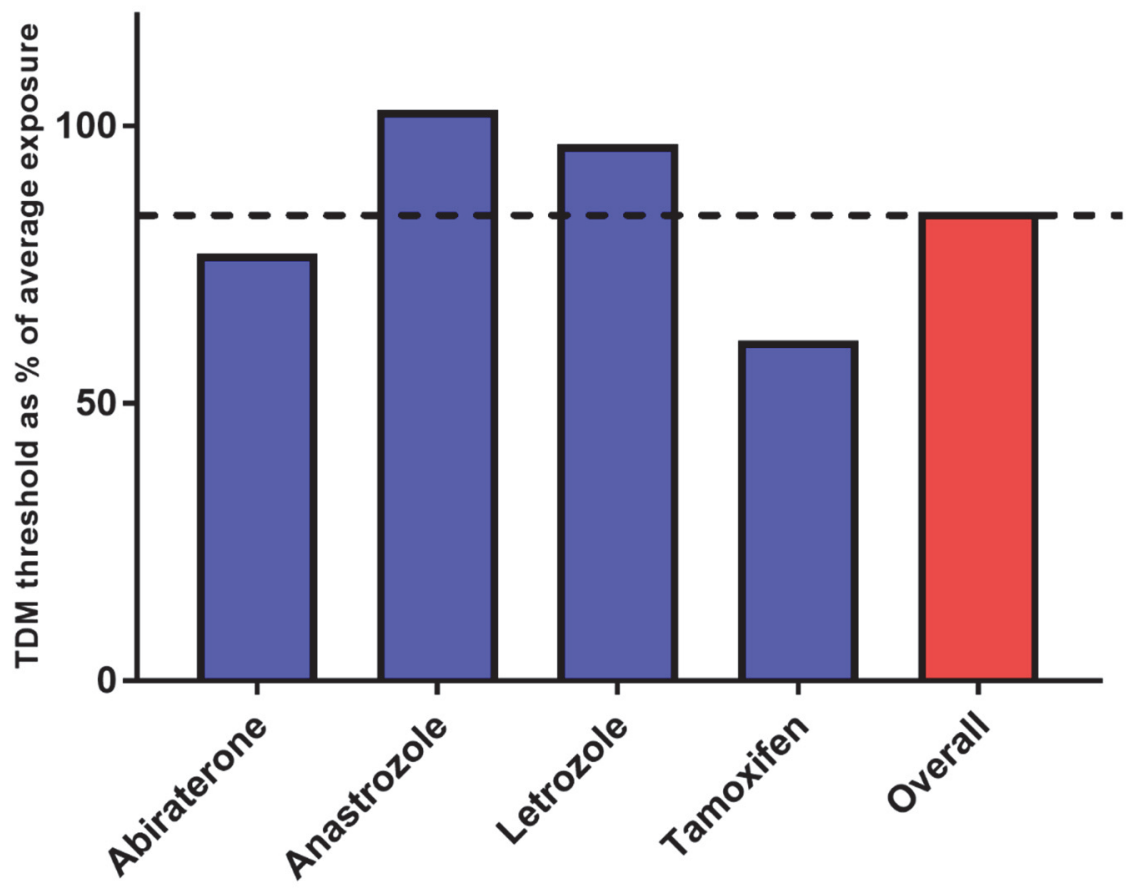

Figure 1 - Proposed therapeutic drug monitoring (TDM) thresholds as percentage of the average exposure

On average the threshold amounted to $85 \%( \pm 19 \%)$ of the population average (indicated by the dotted line).

\section{CONCLUSION}

This review has summarized the clinical PK and PD properties of oral anti-hormonal drugs used in daily oncology practice and aimed to translate these data into practical guidelines for TDM.

For abiraterone, anastrozole, and letrozole, PK targets for TDM could be identified. Furthermore, for tamoxifen, a prospective clinical trial has already demonstrated the feasibility of individualizing the dose based on the endoxifen concentration. However, prospective studies to correlate individualized dosing with tumor response or outcome parameters, such as progression-free survival and overall survival, are lacking.

To conclude, the data presented in this review highlight clear opportunities to study and improve the treatment with oral anti-hormonal agents in oncology via TDM. 


\section{REFERENCES}

1. Siegel RL, Miller KD, Jemal A. Cancer statistics. CA Cancer J Clin 2016; 66: 7-30.

2. Committee for Medicinal Products for Human Use (CHMP). European Medicines Agency. Abiraterone European public assessment report. 2011. Available from: https://www.ema.europa.eu/en/documents/assessment-report/zytiga-epar-publicassessment-report_en.pdf.

3. Verheijen RB, Yu H, Schellens JHM, Beijnen JH, Steeghs N, Huitema ADR. Practical recommendations for therapeutic drug monitoring of kinase inhibitors in oncology. Clin Pharmacol Ther 2017; 102: 765-776.

4. Yu H, Steeghs N, Nijenhuis CM, Schellens JHM, Beijnen JH, Huitema ADR. Practical guidelines for therapeutic drug monitoring of anticancer tyrosine kinase inhibitors: focus on the pharmacokinetic targets. Clin Pharmacokinet 2014; 53: 305-325.

5. Beumer JH. Without therapeutic drug monitoring, there is no personalized cancer care. Clin Pharmacol Ther 2013; 93: 228-230.

6. Paci A, Veal G, Bardin C, Levêque D, Widmer N, Beijnen JH et al. Review of therapeutic drug monitoring of anticancer drugs part 1 - cytotoxics. Eur J Cancer 2014; 50: 2010-2019.

7. Widmer N, Bardin C, Chatelut E, Paci A, Beijnen JH, Levêque D et al. Review of therapeutic drug monitoring of anticancer drugs part two - targeted therapies. Eur J Cancer 2014; 50: 2020-2036.

8. Fox P, Balleine RL, Lee C, Gao B, Balakrishnar B, Menzies AM et al. Dose escalation of tamoxifen in patients with low endoxifen level: evidence for therapeutic drug monitoring the TADE study. Clin Cancer Res 2016; 22: 3164-3171.

9. de Wit D, Guchelaar HJ, den Hartigh J, Gelderblom H, van Erp NP. Individualized dosing of tyrosine kinase inhibitors: are we there yet? Drug Discov Today 2015; 20: 18-36.

10. Food and Drug Administration. Center for Drug Evaluation and Research. Abiraterone Clinical Pharmacology and Biopharmaceutics Review. 2011. Available from: https://www.accessdata.fda.gov/drugsatfda_docs/nda/2011/202379orig1s000clinpharmr.p df

11. James ND, de Bono JS, Spears MR, Clarke NW, Mason MD, Dearnaley DP et al. Abiraterone for prostate cancer not previously treated with hormone therapy. N Engl J Med 2017; 377: 338-351.

12. Ingle JN, Kalari KR, Buzdar AU, Robson ME, Goetz MP, Desta $Z$ et al. Estrogens and their precursors in postmenopausal women with early breast cancer receiving anastrozole. Steroids 2015; 99: 32-38.

13. Food and Drug Administration. Center for Drug Evaluation and Research. Anastrozole Clinical Pharmacology and Biopharmaceutics Review. 2000. Available from: https://www.accessdata.fda.gov/drugsatfda_docs/nda/2000/20541S006_Arimidex_biopharmr.pdf.

14. Plourde P, Dyroff M, Dukes M. Arimidex: a potent and selective fourth-generation aromatase inhibitor. Breast Cancer Res Treat 1994; 30: 103-111.

15. Gervasini G, Jara C, Olier C, Romero N, Martinez R, Carrillo JA. Polymorphisms in ABCB1 and 
CYP19A1 genes affect anastrozole plasma concentrations and clinical outcomes in postmenopausal breast cancer patients. BrJ Clin Pharmacol 2017; 83: 562-571.

16. Dowsett M, Cuzick J, Howell A, Jackson I, ATAC Trialists' Group. Pharmacokinetics of anastrozole and tamoxifen alone, and in combination, during adjuvant endocrine therapy for early breast cancer in postmenopausal women: a sub-protocol of the 'Arimidex ${ }^{\mathrm{TM}}$ and tamoxifen alone or in combination' (ATAC) trial. Br J Cancer 2001; 85: 317-324.

17. Hubalek M, Oberguggenberger A, Beer B, Meraner V, Sztankay M, Oberacher H et al. Does obesity interfere with anastrozole treatment? Positive association between body mass index and anastrozole plasma levels. Clin Breast Cancer 2014; 14: 291-296.

18. Micheal F, Saranya S, Aparna N, Sridevi, Chithra R, Judith MP. Concepts of bioequivalence and it's impact on truncated area under curve (AUC) of drugs with long half life in point estimate and intra-subject variability. J Pharm Sci Res 2012; 4: 1890-1896.

19. Food and Drug Administration. Center for Drug Evaluation and Research. Exemestane Clinical Pharmacology and Biopharmaceutics Review. 1999. Available from: https://www.accessdata.fda.gov/drugsatfda_docs/nda/99/20-

753_Aromasin_biopharmr_P1.pdf.

20. Hertz DL, Kidwell KM, Seewald NJ, Gersch CL, Desta Z, Flockhart DA et al. Polymorphisms in drug-metabolizing enzymes and steady-state exemestane concentration in postmenopausal patients with breast cancer. Pharmacogenomics J 2016; 17: 521-527.

21. Committee for Medicinal Products for Human Use (CHMP). European Medicines Agency. Public Assessment Report Scientific discussion Exemestane. 2010. Available from: https://db.cbg-meb.nl/Pars/h104327.pdf.

22. Food and Drug Administration. Center for Drug Evaluation and Research. Letrozole Clinical Pharmacology and Biopharmaceutics Review. 1997. Available from: https://www.accessdata.fda.gov/drugsatfda_docs/nda/97/20726_FEMARA

2.5MG_BIOPHARMR.PDF.

23. Desta Z, Kreutz Y, Nguyen AT, Li L, Skaar T, Kamdem LK et al. Plasma letrozole concentrations in postmenopausal women with breast cancer are associated with CYP2A6 genetic variants, body mass index, and age. Clin Pharmacol Ther 2011; 90: 693-700.

24. Bisagni G, Cocconi G, Scaglione F, Fraschini F, Pfister C, Trunet PF. Letrozole, a new oral nonsteroidal aromatase inhibitor in treating postmenopausal patients with advanced breast cancer. A pilot study. Ann Oncol 1996; 7: 99-102.

25. Jager NGL, Rosing $\mathrm{H}$, Schellens JHM, Linn SC, Beijnen JH. Tamoxifen dose and serum concentrations of tamoxifen and six of its metabolites in routine clinical outpatient care. Breast Cancer Res Treat 2014; 143: 477-483.

26. Binkhorst L, Kloth JSL, de Wit AS, de Bruijn P, Lam MH, Chaves I et al. Circadian variation in tamoxifen pharmacokinetics in mice and breast cancer patients. Breast Cancer Res Treat 2015; 152: 119-128.

27. Borges S, Desta Z, Li L, Skaar TC, Ward BA, Nguyen A et al. Quantitative effect of CYP2D6 genotype and inhibitors on tamoxifen metabolism: implication for optimization of breast cancer treatment. Clin Pharmacol Ther 2006; 80: 61-74.

28. Mürdter TE, Schroth W, Bacchus-Gerybadze L, Winter S, Heinkele G, Simon W et al. Activity levels of tamoxifen metabolites at the estrogen receptor and the impact of genetic 
polymorphisms of phase $\mathrm{i}$ and ii enzymes on their concentration levels in plasma. Clin Pharmacol Ther 2011; 89: 1-10.

29. Carton E, Noe G, Huillard O, Golmard L, Giroux J, Cessot A et al. Relation between plasma trough concentration of abiraterone and prostate-specific antigen response in metastatic castration-resistant prostate cancer patients. Eur J Cancer 2017; 72: 54-61.

30. Food and Drug Administration. Center for Drug Evaluation and Research. Enzalutamide Clinical Pharmacology and Biopharmaceutics Review. 2012. Available from: http://www.accessdata.fda.gov/drugsatfda_docs/nda/2012/2034150rig1s000ClinPharmR.p df.

31. Wang Y, Chia Y, Nedelman J, Schran H, Mahon F, Molimard M. A therapeutic drug monitoring algorithm for refining the imatinib trough level obtained at different sampling times. Ther Drug Monit 2009; 31: 579-584.

32. Chi KN, Spratlin J, Kollmannsberger C, North S, Pankras C, Gonzalez M et al. Food effects on abiraterone pharmacokinetics in healthy subjects and patients with metastatic castrationresistant prostate cancer. J Clin Pharmacol 2015; 55: 1406-1414.

33. Stuyckens K, Saad F, Xu XS, Ryan CJ, Smith MR, Griffin TW et al. Population pharmacokinetic analysis of abiraterone in chemotherapy-naïve and docetaxel-treated patients with metastatic castration-resistant prostate cancer. Clin Pharmacokinet 2014; 53: 1149-1160.

34. Madlensky L, Natarajan L, Tchu S, Pu M, Mortimer J, Flatt SW et al. Tamoxifen metabolite concentrations, CYP2D6 genotype, and breast cancer outcomes. Clin Pharmacol Ther 2011; 89: 718-725.

35. Food and Drug Administration. Center for Drug Evaluation and Research. Abiraterone Clinical Pharmacology and Biopharmaceutics Review. 2010. Available from: https://www.accessdata.fda.gov/drugsatfda_docs/nda/2011/2023790rig1s000ClinPharmR. pdf.

36. Scher HI, Anand A, Rathkopf D, Shelkey J, Morris MJ, Danila DC et al. Antitumour activity of MDV3100 in castration-resistant prostate cancer: a phase 1-2 study. Lancet 2010; 375: 14371446.

37. Attard G, Reid AHM, Yap TA, Raynaud F, Dowsett M, Settatree S et al. Phase I clinical trial of a selective inhibitor of CYP17, abiraterone acetate, confirms that castration-resistant prostate cancer commonly remains hormone driven. J Clin Oncol 2008; 26: 4563-4571.

38. Xu XS, Ryan CJ, Stuyckens K, Smith MR, Saad F, Griffin TW et al. Modeling the relationship between exposure to abiraterone and prostate-specific antigen dynamics in patients with metastatic castration-resistant prostate cancer. Clin Pharmacokinet 2017; 56: 55-63.

39. Li Z, Bishop AC, Alyamani M, Garcia JA, Dreicer R, Bunch D et al. Conversion of abiraterone to D4A drives anti-tumour activity in prostate cancer. Nature 2015; 523: 347-351.

40. Emamekhoo H, Li Z, Sharifi N. Clinical significance of D4A in prostate cancer therapy with abiraterone. Cell Cycle 2015; 14: 3213-3214.

41. Gibbons JA, Ouatas $T$, Krauwinkel W, Ohtsu Y, van der Walt J-S, Beddo V et al. Clinical Pharmacokinetic Studies of Enzalutamide. Clin Pharmacokinet 2015; 54: 1043-1055.

42. Armstrong AJ, Saad F, Phung D, Dmuchowski C, Shore ND, Fizazi K et al. Clinical outcomes and survival surrogacy studies of prostate-specific antigen declines following enzalutamide in men with metastatic castration-resistant prostate cancer previously treated with 
docetaxel. Cancer 2017; 123: 2303-2311.

43. de Vries Schultink AHM, Zwart W, Linn SC, Beijnen JH, Huitema ADR. Effects of pharmacogenetics on the pharmacokinetics and pharmacodynamics of tamoxifen. Clin Pharmacokinet 2015; 54: 797-810.

44. Jager NGL, Koornstra RHT, Vincent AD, van Schaik RHN, Huitema ADR, Korse CM et al. Hot flashes are not predictive for serum concentrations of tamoxifen and its metabolites. $B M C$ Cancer 2013; 13: 612.

45. Fox P, Balleine RL, Lee C, Gao B, Balakrishnar B, Menzies AM et al. Dose escalation of tamoxifen in patients with low endoxifen level: evidence for therapeutic drug monitoring the TADE study. Clin Cancer Res 2016; 22: 3164-3171.

46. de Vries Schultink AHM, Alexi X, van Werkhoven E, Madlensky L, Natarajan L, Flatt SW et al. An antiestrogenic activity score for tamoxifen and its metabolites is associated with breast cancer outcome. Breast Cancer Res Treat 2016. doi:10.1007/s10549-016-4083-6.

47. Neven P, Jongen L, Lintermans A, Van Asten K, Blomme C, Lambrechts D et al. Tamoxifen metabolism and efficacy in breast cancer- a prospective multicentre trial. Clin Cancer Res 2018; 24: 2312-2318.

48. Jin Y, Desta Z, Stearns V, Ward B, Ho H, Lee KH et al. CYP2D6 genotype, antidepressant use, and tamoxifen metabolism during adjuvant breast cancer treatment. J Natl Cancer Inst 2005; 97: 30-39.

49. Dezentje V, den Hartigh J, Guchelaar H, Hessing T, van der Straaten T, Vletter-Bogaartz J. Association between endoxifen serum concentration and predicted CYP2D6 phenotype in a prospective cohort of patients with early-stage breast cancer. J Clin Oncol 2011; 15(Suppl): 562.

50. Kelly CM, Buzdar AU. Anastrozole. Expert Opin Drug Saf 2010; 9: 995-1003.

51. Pauwels S, Lintermans A, Neven P, Verhaeghe J, Jans I, Billen J et al. Need for estradiol assays with a lower functional sensitivity in clinical studies examining postmenopausal women treated with aromatase inhibitors. J Clin Oncol 2013; 31: 509.

52. Ketha H, Girtman A, Singh RJ. Estradiol assays - the path ahead. Steroids 2015; 99: 39-44.

53. Ingle JN, Buzdar AU, Schaid DJ, Goetz MP, Batzler A, Robson ME et al. Variation in anastrozole metabolism and pharmacodynamics in women with early breast cancer. Cancer Res 2010; 70: 3278-3286.

54. Folkerd EJ, Dixon JM, Renshaw L, A'Hern RP, Dowsett M. Suppression of plasma estrogen levels by letrozole and anastrozole is related to body mass index in patients with breast cancer. J Clin Oncol 2012; 30: 2977-2980.

55. Oberguggenberger A, Meraner V, Sztankay M, Beer B, Weigel G, Oberacher H et al. Can we use gonadotropin plasma concentration as surrogate marker for BMI-related incomplete estrogen suppression in breast cancer patients receiving anastrozole? BMC Cancer 2017; 17: $1-7$.

56. Geisler J, King N, Dowsett M, Ottestad L, Lundgren S, Walton P et al. Influence of anastrozole (Arimidex), a selective, non-steroidal aromatase inhibitor, on in vivo aromatisation and plasma oestrogen levels in postmenopausal women with breast cancer. Br J Cancer 1996; 74: 1286-91. 
57. Mandic S, Kratzsch J, Mandic D, Debeljak Z, Lukic I, Horvat V et al. Falsely elevated serum oestradiol due to exemestane therapy. Ann Clin Biochem An Int J Biochem Lab Med 2017; 54: 402-405.

58. Hertz DL, Speth KA, Kidwell KM, Gersch CL, Desta Z, Storniolo AM et al. Variable aromatase inhibitor plasma concentrations do not correlate with circulating estrogen concentrations in post-menopausal breast cancer patients. Breast Cancer Res Treat 2017; 165: 659-668.

59. de Jonge ME, Huitema ADR, Schellens JHM, Rodenhuis S, Beijnen JH. Individualised cancer chemotherapy: strategies and performance of prospective studies on therapeutic drug monitoring with dose adaptation: a review. Clin Pharmacokinet 2005; 44: 147-173.

60. Food and Drug Administration. Center for Drug Evaluation and Research. Guidance for Industry: Exposure-Response Relationships - Study Design, Data Analysis and Regulatory Applications. 2003. Available from: https://www.fda.gov/regulatory-information/search-fdaguidance-documents/exposure-response-relationships-study-design-data-analysis-andregulatory-applications.

61. van Nuland M, Hillebrand MJX, Rosing H, Schellens JHM, Beijnen JH. Development and validation of an LC-MS/MS method for the simultaneous quantification of abiraterone, enzalutamide, and their major metabolites in human plasma. Ther Drug Monit 2017; 39: 243251.

62. de Krou S, Rosing $H$, Nuijen B, Schellens JHM, Beijnen JH. Fast and adequate liquid chromatography-tandem mass spectrometric determination of z-endoxifen serum levels for therapeutic drug monitoring. Ther Drug Monit 2017; 39: 132-137.

63. van Nuland M, Rosing H, de Vries J, Ovaa H, Schellens JHM, Beijnen JH. An LC-MS/MS method for quantification of the active abiraterone metabolite $\triangle(4)$-abiraterone (D4A) in human plasma. J Chromatogr B Anal Technol Biomed Life Sci 2017; 1068-1069: 119-124.

64. Shao R, Yu L, Lou H, Ruan Z, Jiang B, Chen J. Development and validation of a rapid LC-MS/MS method to quantify letrozole in human plasma and its application to therapeutic drug monitoring. Biomed Chromatogr 2016; 30: 632-637.

65. Yu J, He J, Zhang Y, Qin F, Xiong Z, Li F. Development of a liquid chromatography-tandem mass spectrometry method for determination of butoconazole nitrate in human plasma and its application to a pharmacokinetic study. Biomed Chromatogr 2011; 25: 511-516.

66. Wang L-Z, Goh S-H, Wong AL-A, Thuya W-L, Lau J-YA, Wan S-C et al. Validation of a rapid and sensitive LC-MS/MS method for determination of exemestane and its metabolites, 17betahydroxyexemestane and 17beta-hydroxyexemestane-17-O-beta-D-glucuronide: application to human pharmacokinetics study. PLoS One 2015; 10: e0118553.

67. Cardoso E, Csajka C, Schneider MP, Widmer N. Effect of adherence on pharmacokinetic/pharmacodynamic relationships of oral targeted anticancer drugs. Clin Pharmacokinet 2018; 57: 1-6. 



\section{PART IV}

Alternative strategies for precision dosing 



\section{ABSTRACT}

\section{Aim}

Abiraterone acetate is approved for the treatment of metastatic prostate cancer. At the currently used fixed dose of $1000 \mathrm{mg}$ once daily in modified fasting state, $40 \%$ of patients do not reach the efficacy threshold of a minimum plasma concentration $\left(C_{\min }\right) \geq 8.4 \mathrm{ng} / \mathrm{mL}$ and are thereby at risk of decreased treatment efficacy. This study aims to evaluate whether pharmacokinetically (PK) guided abiraterone acetate dosing with a food intervention is feasible and results in an increased percentage of patients with concentrations above the target.

\section{Methods}

Patients starting regular treatment with abiraterone acetate in modified fasting state were included. Pharmacokinetic analysis was performed 4, 8 and 12 weeks after start of treatment and every 12 weeks thereafter. In case of $C_{\min }<8.4 \mathrm{ng} / \mathrm{mL}$ and acceptable toxicity, a PK-guided intervention was recommended. The first step was concomitant intake of abiraterone acetate with a light meal or a snack.

\section{Results}

In total, 32 evaluable patients were included, of which 20 patients (63\%) had a $C_{\min }<8.4$ $\mathrm{ng} / \mathrm{mL}$ at a certain time point during treatment. These patients were recommended to take abiraterone acetate concomitantly with food, after which $C_{\text {min }}$ increased from 6.9 $\mathrm{ng} / \mathrm{mL}$ to $27 \mathrm{ng} / \mathrm{mL}(\mathrm{p}<0.001)$ without additional toxicities. This intervention led to adequate exposure in 28 patients (87.5\%).

\section{Conclusion}

Therapeutic drug monitoring of abiraterone was applied in clinical practice and proved to be feasible. Concomitant intake with food resulted in a significant increase in $C_{\text {min }}$ and offers a cost-neutral opportunity to optimize exposure in patients with low $C_{\min }$. 


\section{INTRODUCTION}

Abiraterone acetate is an antihormonal prodrug, which is rapidly converted to its active form abiraterone after oral ingestion. Abiraterone inhibits 17a-hydroxylase/C17,20-lyase (CYP17) and thereby blocks the androgen biosynthesis. Initially, abiraterone acetate was approved for the treatment of metastatic castration-resistant prostate cancer, but recently, it has also been approved for the treatment of metastatic hormone-sensitive prostate cancer. $^{1}$

Exposure-response analyses have shown that plasma concentrations of abiraterone are related to efficacy. ${ }^{2-4}$ Carton et al. demonstrated that progression-free survival (PFS) was significantly longer in patients with a minimum plasma concentration $\left(C_{\min }\right)$ above 8.4 $\mathrm{ng} / \mathrm{mL}$ compared with those below (12.2 vs. 7.4 months, $\mathrm{p}=0.044){ }^{3}$ We have confirmed this exposure-efficacy threshold in a real-life patient cohort. ${ }^{4}$

Abiraterone acetate is currently administered using a one-size-fits-all approach, in which all patients receive a dose of $1000 \mathrm{mg}$ once daily (QD) without food. This dosing strategy results in high interindividual variability in exposure to abiraterone, with a coefficient of variation (CV\%) of $46-70 \%$ for $C_{\text {min. }}{ }^{3,4}$ At the currently used fixed dose, $35-42 \%$ of patients do not reach the efficacy threshold of $C_{\min } \geq 8.4 \mathrm{ng} / \mathrm{mL}$ and are thus underdosed. ${ }^{3,4}$ This provides a strong rationale for therapeutic drug monitoring (TDM) to intervene and to increase the number of patients having an adequate abiraterone exposure.

As food intake impacts the absorption of abiraterone, concomitant intake of abiraterone acetate and food could be applied in case of low exposure. According to the drug label ${ }^{5}$, abiraterone acetate should be administered in a modified fasting state, which means no food $2 \mathrm{~h}$ before and $1 \mathrm{~h}$ after intake of the drug. However, concomitant intake with food has been shown to result in a clinically relevant increase in exposure in a previous foodeffect study. ${ }^{6}$

The aim of this study was to evaluate whether TDM of abiraterone with a food intervention is feasible in clinical practice and results in an increased percentage of patients with efficacious exposure to abiraterone without additional toxicities.

\section{METHODS}

\section{Patients}

Patients starting regular treatment with abiraterone acetate at the registered dose of $1000 \mathrm{mg}$ QD in a modified fasting state were included in an ongoing prospective study on TDM of oral anticancer drugs (www.trialregister.nl; NL6695). ${ }^{7}$ 


\section{Objectives}

The primary objective of this study was to halve the percentage of patients with an exposure below the target of $8.4 \mathrm{ng} / \mathrm{mL}$ after 12 weeks compared to historical data. The study of Carton et al. was taken as a reference, in which $35 \%$ of patients had a mean $\mathrm{C}_{\text {min }}$ $<8.4 \mathrm{ng} / \mathrm{mL}^{3}$ Secondary objectives were to evaluate the feasibility, tolerability and efficacy of TDM of abiraterone with a food intervention in clinical practice and to achieve a physician adherence $>90 \%$ (i.e. whether TDM recommendations were followed by the treating physician). Feasibility was defined as the percentage of successful pharmacokinetically (PK) guided interventions (i.e. target attainment without additional toxicities). Tolerability was evaluated by the incidence of clinically relevant toxicities, defined as toxicities leading to dose reduction, treatment interruption or discontinuation, as evaluated by the treating physician. Preliminary efficacy was assessed by comparing PFS and prostate-specific antigen (PSA) responses between patients who needed a PKguided intervention and those who did not (i.e. all $C_{\min } \geq 8.4 \mathrm{ng} / \mathrm{mL}$ ). PFS was defined as the time from start of treatment to progression, as assessed by the treating physician based on either PSA increase, radiological progression or clinical progression. PSA response was defined as $\geq 50 \%$ decrease in PSA from baseline, according to the Prostate Cancer Working Group 2 criteria. 8,9

\section{PK samples}

PK samples were collected 4, 8 and 12 weeks after start of treatment and every 12 weeks thereafter. Figure 1 provides an overview of the study design. Abiraterone concentrations were measured using a validated liquid chromatography-tandem mass spectrometry assay. ${ }^{10} \mathrm{C}_{\mathrm{min}}$ was estimated using the following formula:

$$
C_{\text {min }}=C_{\text {measured }} * 0.5 \frac{\text { dosing interval }-T A D}{t_{1 / 2}}
$$

in which $C_{\text {measured }}$ is the measured plasma concentration, dosing interval is the time between two consecutive administrations of the drug (i.e. $24 \mathrm{~h}$ ), TAD is the time after dose (i.e. time between last intake of the drug and collection of the PK sample) and $t_{1 / 2}$ is the elimination half-life of the drug (i.e. $12 \mathrm{~h}^{11}$ ).

\section{PK-guided interventions}

In case of $\mathrm{C}_{\min }<8.4 \mathrm{ng} / \mathrm{mL}$ and acceptable toxicity, a PK-guided intervention was recommended. After compliance and drug-drug interactions were checked, the first step was concomitant intake of abiraterone acetate with a light meal or a snack. No specified meals were used. Patients were instructed to take abiraterone acetate for example with some bread, yoghurt or fruit, but not with food high in fat. If exposure remained below the target, dose increments of abiraterone acetate were recommended (to 1250 and 1500 $\mathrm{mg}$, respectively). Dose reductions were solely based on toxicities, not on exposure. 


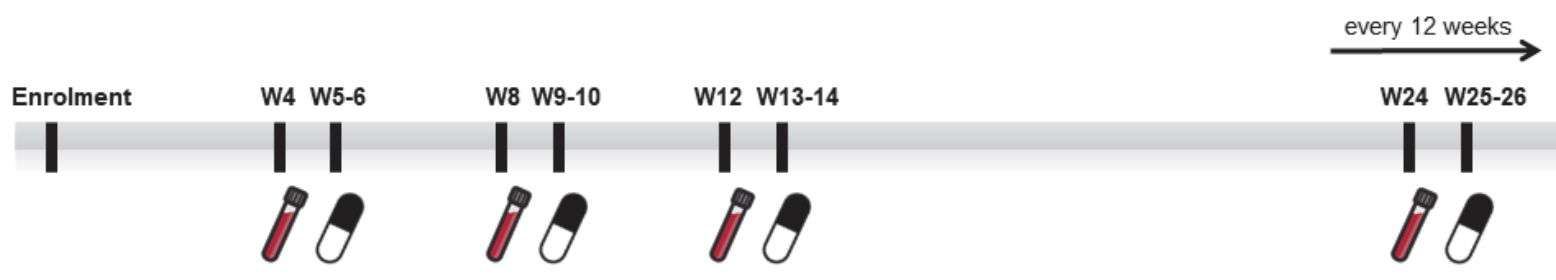

PK samples were drawn 4,8 and 12 weeks after start of treatment and every 12 weeks thereafter

PK-guided interventions were recommended within 1-2 weeks and included emphasising compliance, adjusting concomitant medication (due to drug-drug interactions), concomitant intake of abiraterone acetate with a light meal or a snack or conventional dose increments of abiraterone acetate

Figure 1 - Schematic overview of study design

$P K=$ pharmacokinetic(ally); $W=$ week

\section{Statistical analyses}

Patients were evaluable for the primary endpoint if they completed the first three PK measurements. The effect of concomitant intake of abiraterone acetate and food was evaluated by a Wilcoxon signed-rank test and a Mann-Whitney $U$ test. Preliminary efficacy was evaluated using univariable and multivariable Cox regression and logistic regression analyses. Other data were analysed using descriptive statistics. Statistical analyses were performed using R version 3.3.2. ${ }^{12}$

\section{Ethical regulations}

This study was assessed by the accredited Medical Ethics Committee of the Netherlands Cancer Institute, Amsterdam, The Netherlands, in May 2017, and it was reviewed not to fall under the Dutch Medical Research Involving Human Subjects Act, because TDM is performed as standard care, and no additional procedures were required for participants. The study was authorized by the institutional review board. Patients provided written informed consent. The study protocol followed the principles of the Declaration of Helsinki.

\section{RESULTS}

\section{Patient characteristics}

In total, 32 evaluable patients were enrolled in the study between June 2017 and December 2018 (Figure 2). Baseline characteristics of these patients are provided in Table 1. Twenty-nine patients completed the first three PK measurements and were eligible for evaluation of the primary endpoint. Twenty patients (63\%) had $C_{\min }<8.4 \mathrm{ng} / \mathrm{mL}$ at a certain time point during treatment. In general, these patients tended to have received more prior lines of treatment, had a worse World Health Organisation (WHO) performance status and had a higher baseline PSA compared with patients with all $C_{\min } \geq$ 
$8.4 \mathrm{ng} / \mathrm{mL}$. At the time of data cut-off (30 August 2019), 13 patients (41\%) were still on treatment with a median duration of 11.4 months (range: 2.8-26.3 months).

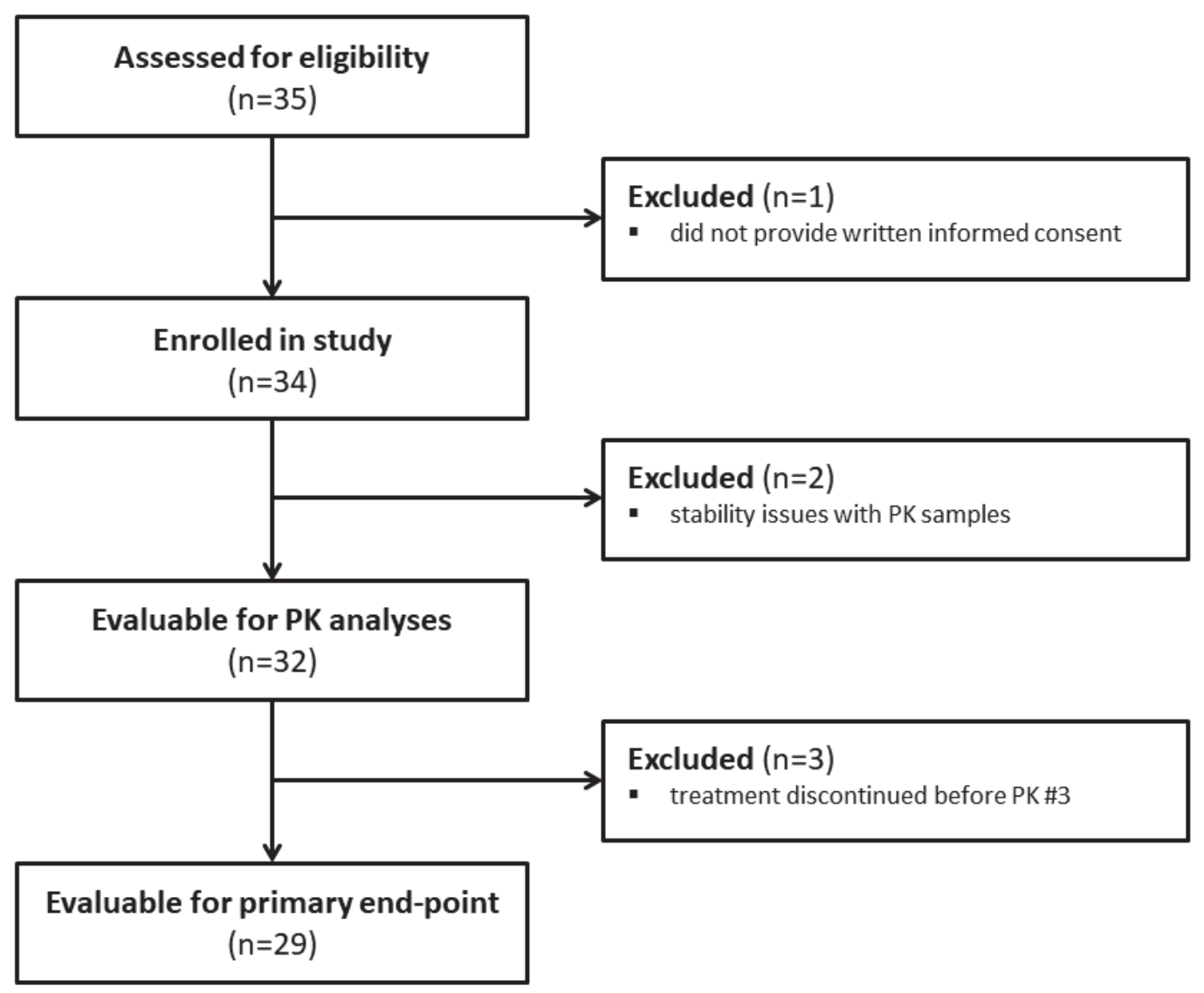

Figure 2 - Patient flow chart

$P K=$ pharmacokinetic

\section{Pharmacokinetically guided dosing}

In total, 194 samples have been collected, with a median number of samples per patient of 6 (range: 1-13). First, the results with regard to the primary outcome will be described (i.e. to halve the percentage of patients with a low exposure after 12 weeks). An overview of the median $C_{\min }$ and the percentage of patients with $C_{\min }$ below the efficacy threshold at each time point can be found in Table 2 . After 4 weeks of abiraterone acetate treatment at $1000 \mathrm{mg}$ QD in modified fasting state, median abiraterone $C_{\min }$ was $12.5 \mathrm{ng} / \mathrm{mL}$ (range: 1.0-100 ng/mL), and 8 patients (25\%) had a $C_{\min }<8.4 \mathrm{ng} / \mathrm{mL}$. After 12 weeks, median abiraterone $C_{\min }$ increased to $17 \mathrm{ng} / \mathrm{mL}$ (range: $6.7-126 \mathrm{ng} / \mathrm{mL}$ ) after a food intervention was implemented in patients with a low exposure, with $10 \%$ of patients not reaching the target.

To evaluate the secondary objectives, all PK-guided interventions were taken into account, so also the interventions that were performed after 12 weeks. Figure 3 provides an overview of the PK-guided interventions and its results. The 20 patients (63\%) with $C_{\min }<$ $8.4 \mathrm{ng} / \mathrm{mL}$ at a certain time point during treatment were recommended to take 
abiraterone acetate concomitantly with a light meal or a snack. In one patient, this PKguided intervention could not be performed, because treatment was discontinued because of progression. The interventions resulted in adequate exposure (i.e. $C_{\min } \geq 8.4$ $\mathrm{ng} / \mathrm{mL}$ ) in 16 patients (84\%). In two patients, the effect could not be evaluated, as treatment was discontinued because of progression before the next PK measurement. In one patient, $C_{\min }$ remained below the target initially, and further dose escalation was not deemed feasible because of prior liver toxicity. Eventually, the target was reached with the initial recommended intake of food. Physician adherence to the recommendations was $100 \%$. In total, 28 patients (87.5\%) eventually had an adequate exposure. Only one patient (3\%) had a median $\mathrm{C}_{\min }<8.4 \mathrm{ng} / \mathrm{mL}$.

\section{Table 1 - Baseline characteristics}

\begin{tabular}{|c|c|c|c|}
\hline Characteristic & $\begin{array}{l}\text { Patients with } \geq 1 \\
\text { measurement of } \\
\text { abiraterone } C_{\min }< \\
8.4 \mathrm{ng} / \mathrm{mL}(\mathrm{n}=20)\end{array}$ & $\begin{array}{l}\text { Patients with all } \\
\text { measurements of } \\
\text { abiraterone } C_{\min } \geq \\
8.4 \mathrm{ng} / \mathrm{mL}(n=12)\end{array}$ & $\begin{array}{l}\text { All evaluable } \\
\text { patients } \\
(n=32)\end{array}$ \\
\hline Age (years) & $73[52-87]$ & 73 [63-83] & $73[52-87]$ \\
\hline \multicolumn{4}{|c|}{ WHO performance status } \\
\hline 0 & $3(15 \%)$ & $5(42 \%)$ & $8(25 \%)$ \\
\hline 1 & $11(55 \%)$ & $6(50 \%)$ & $17(53 \%)$ \\
\hline 2 & $5(25 \%)$ & $1(8 \%)$ & $6(19 \%)$ \\
\hline 3 & $1(5 \%)$ & 0 & $1(3 \%)$ \\
\hline \multicolumn{4}{|l|}{ Treatment setting } \\
\hline Castration-resistant & $19(95 \%)$ & $12(100 \%)$ & $31(97 \%)$ \\
\hline Hormone-sensitive & $1(5 \%)$ & 0 & $1(3 \%)$ \\
\hline \multicolumn{4}{|c|}{$\begin{array}{l}\text { Previous lines of systemic } \\
\text { treatment }^{a}\end{array}$} \\
\hline 0 & $11(55 \%)$ & $9(75 \%)$ & $20(63 \%)$ \\
\hline 1 & $4(20 \%)$ & $2(17 \%)$ & $6(19 \%)$ \\
\hline$\geq 2$ & $5(25 \%)$ & $1(8 \%)$ & $6(19 \%)$ \\
\hline \multicolumn{4}{|c|}{ Previous systemic treatment $^{a}$} \\
\hline Docetaxel & $9(45 \%)$ & $2(17 \%)$ & $11(34 \%)$ \\
\hline Enzalutamide & $3(15 \%)$ & $1(8 \%)$ & $4(13 \%)$ \\
\hline Radium-223 & $3(15 \%)$ & $1(8 \%)$ & $4(13 \%)$ \\
\hline Cabazitaxel & $4(20 \%)$ & 0 & $4(13 \%)$ \\
\hline \multicolumn{4}{|l|}{ Gleason score } \\
\hline$\leq 7$ & $10(50 \%)$ & $7(58 \%)$ & $17(53 \%)$ \\
\hline 8-10 & $9(45 \%)$ & $5(42 \%)$ & $14(44 \%)$ \\
\hline Missing & $1(5 \%)$ & 0 & $1(3 \%)$ \\
\hline Baseline PSA (ng/mL) & $83[6-1036]$ & $32[6-282]$ & $48[6-1036]$ \\
\hline
\end{tabular}

Data are expressed as no. (\%) or median [range], as appropriate.

a in castration-resistant setting

$C_{\min }=$ minimum plasma concentration; $P S A=$ prostate specific antigen 
Table 2 - Abiraterone $C_{\min }$ and percentage of patients with low pharmacokinetic exposure after 4,8 and 12 weeks

\begin{tabular}{cl}
\hline Parameter & Result \\
\hline Abiraterone $\mathbf{C}_{\min }$ & in $\mathbf{n g} / \mathbf{m L}$ [range] \\
PK sample \#1 (week 4) & $13[1.0-100]$ \\
PK sample \#2 (week 8) & $17[5.8-114]$ \\
PK sample \#3 (week 12) & $17[6.7-126]$ \\
\hline Patients with $\mathbf{C}_{\min }$ below the target of $8.4 \mathbf{~ n g / m L}$ & $\mathbf{n}(\%)$ \\
PK sample \#1 (week 4) & $8(25 \%)$ \\
PK sample \#2 (week 8) & $6(19 \%)$ \\
PK sample \#3 (week 12) & $3(10 \%)$ \\
Any time point during treatment & $20(63 \%)$ \\
\hline
\end{tabular}

Data are expressed as median [range] or number (\%), as appropriate.

PK\#1: 32 patients; PK\#2: 31 patients; PK\#3: 29 patients.

$C_{\text {min }}=$ minimum plasma concentration; $P K=$ pharmacokinetic

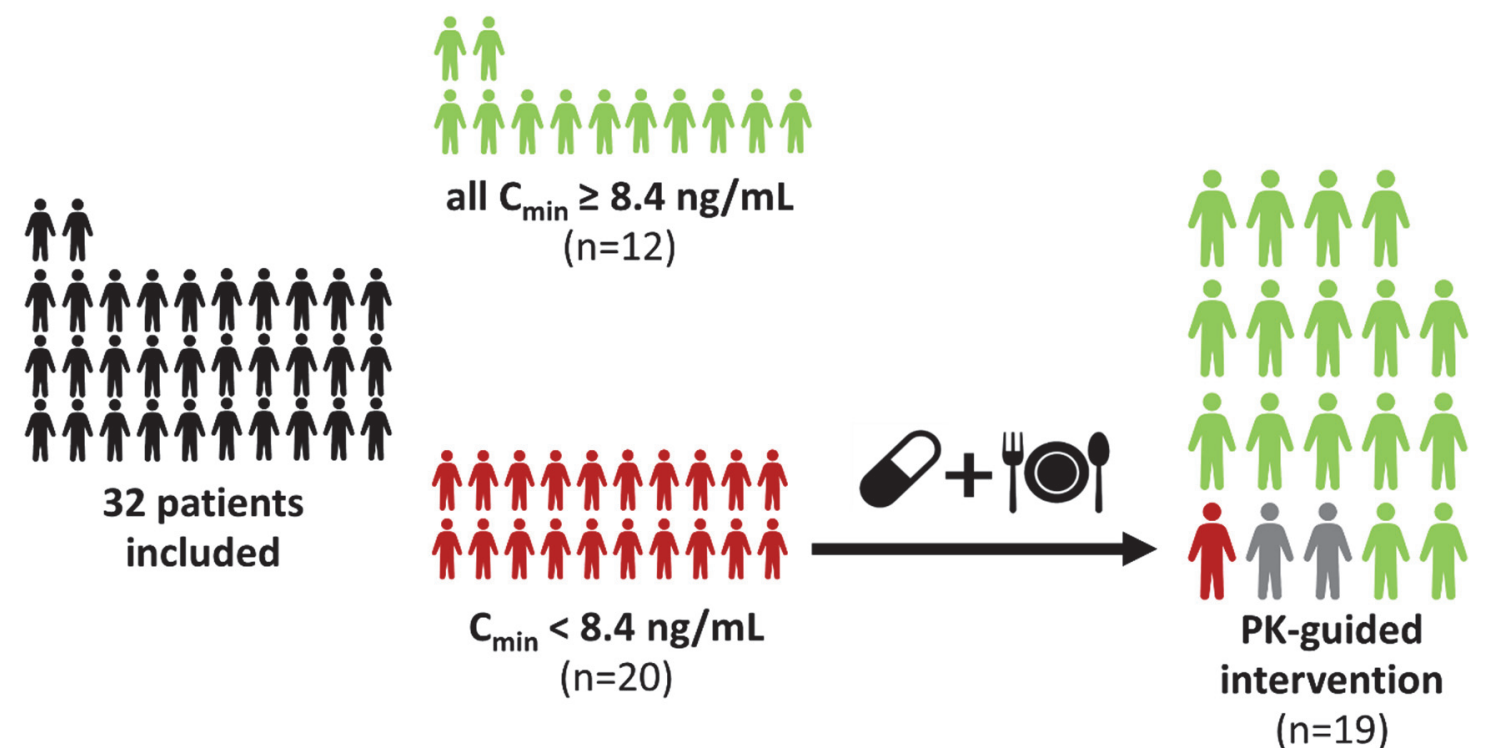

Figure 3 - Schematic overview of study results

$(n=19)$

The group of patients with $C_{\min }<8.4 \mathrm{ng} / \mathrm{mL}(\mathrm{n}=20)$ had one or more PK-samples with a calculated $\mathrm{C}_{\min }<8.4 \mathrm{ng} / \mathrm{mL}$ at a certain time point during their treatment. In one patient, a PK-guided intervention could not be performed, because treatment was discontinued because of progressive disease. In two patients, the effect of the PK-guided intervention could not be evaluated, because treatment was discontinued because of progressive disease before the next PK measurement. In one patient, the PK-guided intervention did not result in $C_{\min } \geq 8.4 \mathrm{ng} / \mathrm{mL}$, further dose escalation was not deemed feasible because of prior liver toxicity.

$C_{\min }=$ minimum plasma concentration; $P K=$ pharmacokinetically

Figure 4 shows boxplots of abiraterone $C_{\min }$ in patients with adequate and low exposure, before and after concomitant intake with food. In the group of patients with adequate exposure (i.e. all $C_{\min } \geq 8.4 \mathrm{ng} / \mathrm{mL}$ ), in which no PK-guided intervention was needed, median abiraterone $C_{\min }$ was $23 \mathrm{ng} / \mathrm{mL}$ (range: 15-70 ng/mL). In the group with low exposure (i.e. $C_{\min }<8.4 \mathrm{ng} / \mathrm{mL}$ ), median abiraterone $C_{\min }$ before the PK-guided 
intervention was $6.9 \mathrm{ng} / \mathrm{mL}$ (range: $1.0-8.2 \mathrm{ng} / \mathrm{mL}$ ). Concomitant intake of abiraterone acetate and food resulted in an increase in $C_{\min }$ to $27 \mathrm{ng} / \mathrm{mL}$ (range: $4.3-94 \mathrm{ng} / \mathrm{mL}$, p < 0.001), which was comparable to the patients with all $C_{\min }$ above the target.

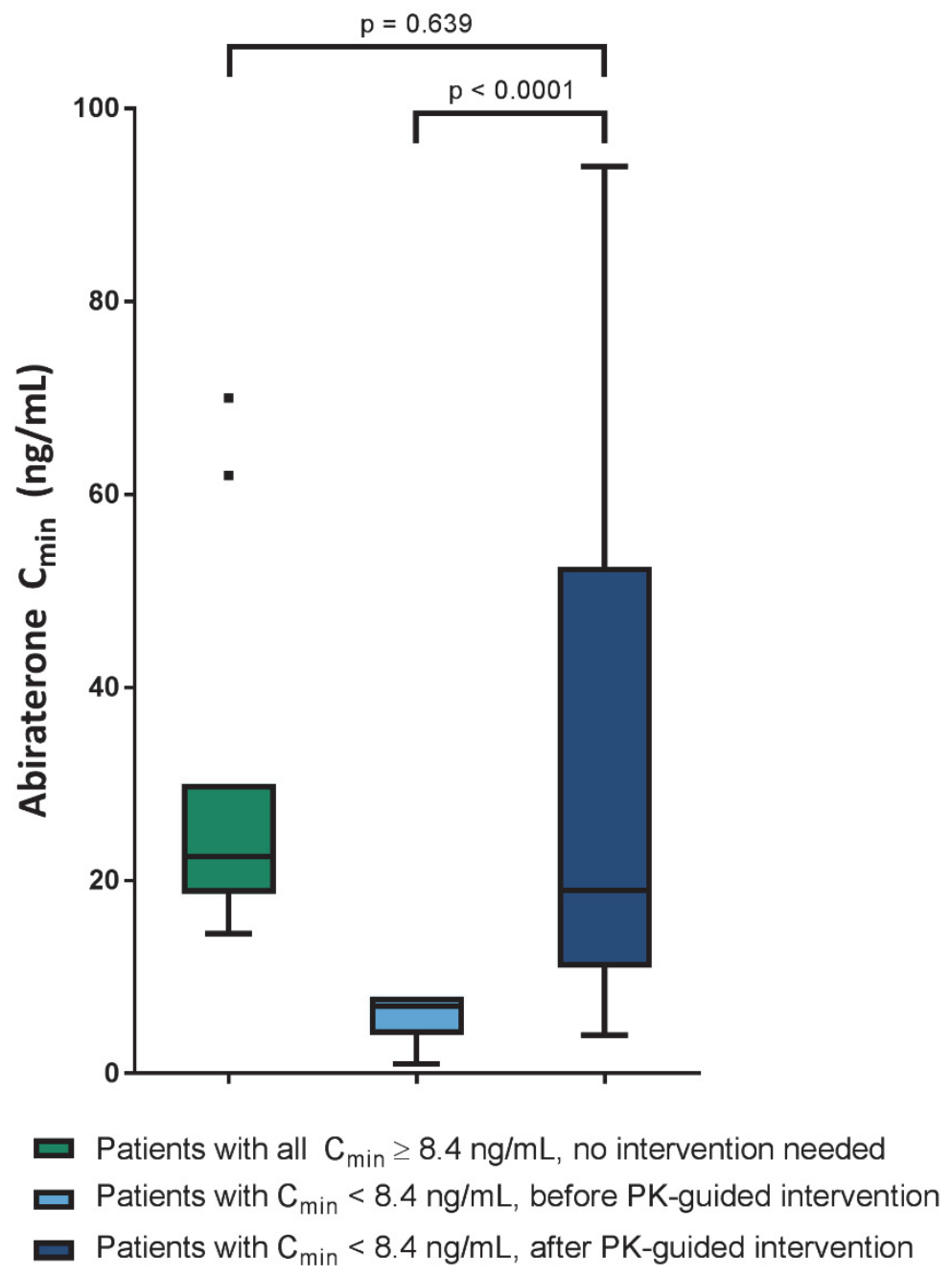

Figure 4 - Box plots of abiraterone $C_{\min }$ in patients with adequate and low pharmacokinetic exposure, before and after concomitant intake with food

$C_{\text {min }}=$ minimum plasma concentration; $P K=$ pharmacokinetically

For the patients who had a $\mathrm{C}_{\min }<8.4 \mathrm{ng} / \mathrm{mL}$ at a later time point during treatment, median $\mathrm{C}_{\text {min }}$ in previous $\mathrm{PK}$ samples was $14 \mathrm{ng} / \mathrm{mL}$ (range: 9.0-77 $\mathrm{ng} / \mathrm{mL}$ ) with a median intraindividual variability (CV\%) of $23 \%$.

In Supplementary Figure 1, individual graphs of the patients who received a food intervention are shown, depicting all measured abiraterone concentrations before and after concomitant intake with a light meal or a snack.

\section{Toxicity}

Three patients needed a dose reduction to $500 \mathrm{mg}$ QD because of toxicity (elevated liver enzymes $(n=2)$ and fatigue $(n=1))$. None of these patients had received a PK-guided 
intervention when toxicity emerged. Median $C_{\min }$ at presentation was $33 \mathrm{ng} / \mathrm{mL}$ (range: 11-48 ng/mL). After dose reduction, exposure remained adequate in two patients. In one patient, $C_{\text {min }}$ dropped below the target, after which the dose was carefully increased to 1000 mg QD concomitant with food, and the target was reached eventually.

In the patients who did receive a PK-guided intervention, this did not lead to additional toxicities.

\section{Efficacy}

Median PFS was 9.3 months (95\% confidence interval (CI): 6.8-NA) in patients with one or more $\mathrm{C}_{\min }<8.4 \mathrm{ng} / \mathrm{mL}$, compared with not reached yet $(95 \% \mathrm{Cl}$ : 15.8-NA) in patients with all $\mathrm{C}_{\min } \geq 8.4 \mathrm{ng} / \mathrm{mL}$ (hazard ratio: $2.59,95 \% \mathrm{Cl}: 0.84-7.97, \mathrm{p}=0.097$ ). However, in multivariable Cox regression, $C_{\min }<8.4 \mathrm{ng} / \mathrm{mL}$ resulted in a hazard ratio of $1.14(95 \% \mathrm{Cl}$ : 0.34-3.85, $p=0.834$ ), when WHO performance status and number of prior lines of treatment were taken into account. In five patients, the last $C_{\min }$ before progression was $<8.4 \mathrm{ng} / \mathrm{mL}$ (three of them received successful PK-guided interventions before).

In the initially low $\mathrm{C}_{\min }$ cohort, 10 patients had a PSA response (50\%), whereas in the group with all $C_{\min } \geq 8.4 \mathrm{ng} / \mathrm{mL}, 11$ patients $(92 \%)$ had a PSA response. Multivariable logistic regression resulted in an odds ratio of 0.15 for $C_{\min }<8.4 \mathrm{ng} / \mathrm{mL}(p=0.154)$.

\section{DISCUSSION}

In this prospective study we evaluated the feasibility of PK-guided abiraterone acetate dosing. At the authorized dose of $1000 \mathrm{mg}$ QD in modified fasting state, $63 \%$ of patients had a $\mathrm{C}_{\mathrm{min}}<8.4 \mathrm{ng} / \mathrm{mL}$ at a certain time point during treatment. Concomitant intake with a light meal or a snack in these patients resulted in a 3.8-fold increase in $C_{\text {min }}$ without additional toxicities (Figures 3 and 4; Table 2). Hence, TDM of abiraterone is feasible and concomitant intake with food offers a strategy to optimize exposure in patients with a low $\mathrm{C}_{\min }$. For the small proportion of patients in whom the target is not attained with this food intervention, a dose increase can be recommended, although this has not been the case in this study.

In our study, $63 \%$ of patients had a low exposure at a certain time point during treatment, which is notably higher than the $35-42 \%$ reported in literature. ${ }^{3,4}$ However, these values refer to the mean or median value of multiple abiraterone $C_{\min }$ measurements, whereas in our study, it represents every patient with a single measurement below the target. In our cohort, only one patient (3\%) had a median $C_{\min }$ below $8.4 \mathrm{ng} / \mathrm{mL}$ because of the successful PK-guided interventions. It is remarkable that especially patients with more prior lines of treatment appear to be at risk of low exposure, which was also seen in our previous exposure-response analysis for abiraterone. ${ }^{4}$ It would be of interest to further 
investigate the mechanism behind the lower exposure in this subgroup (e.g. higher clearance because of enzyme induction or decreased absorption).

Concomitant intake with food not only resulted in an increased exposure but also led to a considerably higher interindividual variability (Figure 4). As a result, some patients attained very high $C_{\min }$ levels. This may be attributed to the fact that meals were not specified and that the composition could thus differ between patients and time points. However, no additional toxicities were experienced by these patients, which is in line with previous literature where no exposure-toxicity relationship was found either. ${ }^{1,3,4}$ Therefore, the increased interindividual variability in exposure is considered acceptable, as long as $C_{\min }$ levels are above $8.4 \mathrm{ng} / \mathrm{mL}$.

Since abiraterone also shows a high intra-individual variability, many patients had a $C_{\text {min }}$ below $8.4 \mathrm{ng} / \mathrm{mL}$ at a later time point during treatment. From that moment, patients were recommended to take abiraterone acetate concomitantly with food, whereas it was uncertain if this would have been necessary all the time. However, owing to the absence of an exposure-toxicity relationship, long-term implementation of this PK-guided intervention does not appear to be harmful.

The magnitude of the food effect in our study is not in line with the previous study by Chi et $a l .{ }^{6}$ While they found a similar exposure (i.e. area under the concentration-time curve (AUC)) for a low-fat meal compared with modified fasting state, our study shows a 3.8-fold increase in $C_{\min }$ after concomitant intake with a light meal or a snack. A possible explanation for this could be that many patients (65\%) took abiraterone acetate early in the morning, which was probably after an overnight fast. In that case, the results would be more consistent with the study of Chi et al., who reported a five-fold increase in AUC for a low-fat meal compared with overnight fasting in healthy volunteers. ${ }^{6}$

Compared with conventional dose increments, concomitant intake with food offers a costneutral strategy to increase pharmacokinetic exposure, although a longer treatment duration could result in higher total treatment costs. Additional costs for a $250 \mathrm{mg}$ or 500 mg dose increase would be $€ 862$ or $€ 1782$, respectively, per patient per month in The Netherlands. Furthermore, concomitant intake with food is more patient-friendly because patients do not have to take into account the modified fasting conditions.

This prospective study provides real-life data on a TDM programme. Advantages of this study design include the fact that data are representative for the abiraterone population in clinical practice and that our findings can easily be implemented in routine care. On the other hand, this is simultaneously a limitation of our study because compliance could not be guaranteed (i.e. no drug accountability has been performed, and no patient diaries were used). 
Although this study demonstrated that an adequate exposure could be attained in the majority of patients by the support of TDM, the ultimate goal is to improve treatment efficacy. Preliminary data on efficacy in this small group of patients indicate that patients who needed a PK-guided intervention still have a shorter PFS than patients with all adequate $C_{\min }$. However, patients with a low $C_{\min }$ had a less favourable prognosis at baseline, as they received more prior lines of treatment, had a worse WHO performance status and a higher baseline PSA. We have statistically shown that the adverse results in this cohort are influenced by the adverse patient characteristics in the initially low $C_{\text {min }}$ cohort. To evaluate whether TDM actually improves treatment outcomes, a larger cohort of patients will be needed. Therefore, patient inclusion in this study will continue to investigate the effect on treatment efficacy as well.

The significant food effect of abiraterone raises two other interesting concepts. The first is a cost-saving approach: treating patients at a lower dose with food, as has been evaluated by Szmulewitz et al. ${ }^{13}$ Ideally, this would be investigated using a two-step procedure. To start, it should be proven that adding food to efficiently raise $C_{\min }$ is associated with better treatment outcomes for the standard dose of abiraterone. Then, the same should be proven for lower doses of abiraterone. The other concept is a more pragmatic approach: to recommend concomitant intake with food to all patients, regardless of pharmacokinetic exposure.

In conclusion, we demonstrated that TDM of abiraterone is feasible in clinical practice. Furthermore, concomitant intake of abiraterone acetate and food resulted in a significant increase in $C_{\min }$ and thereby offers a safe and cost-neutral opportunity to optimize exposure in patients with a low $C_{\text {min }}$. Therefore, we recommend to implement TDM of abiraterone for all patients in routine care.

\section{ACKNOWLEDGEMENTS}

The authors would like to thank all patients for their participation in this study. This work was supported by unrestricted research grants from Novartis, Pfizer and Roche. The funding sources had no involvement in the study design, collection, analysis and interpretation of data, the writing of the report or in the decision to submit the article for publication. This work was presented in part at the 2019 ASCO (American Society of Clinical Oncology) Annual Meeting, Chicago (J Clin Oncol 37, 2019 (suppl; abstr 3117)). 


\section{REFERENCES}

1. Committee for Medicinal Products for Human Use (CHMP). European Medicines Agency. Abiraterone European Public Assessment Report. 2011. Available from: https://www.ema.europa.eu/en/documents/assessment-report/zytiga-epar-publicassessment-report_en.pdf.

2. Xu XS, Ryan CJ, Stuyckens K, Smith MR, Saad F, Griffin TW et al. Modeling the relationship between exposure to abiraterone and prostate-specific antigen dynamics in patients with metastatic castration-resistant prostate cancer. Clin Pharmacokinet 2017; 56: 55-63.

3. Carton E, Noe G, Huillard O, Golmard L, Giroux J, Cessot A et al. Relation between plasma trough concentration of abiraterone and prostate-specific antigen response in metastatic castration-resistant prostate cancer patients. Eur J Cancer 2017; 72: 54-61.

4. van Nuland $\mathrm{M}$, Groenland $\mathrm{SL}$, Bergman AM, Steeghs $\mathrm{N}$, Rosing $\mathrm{H}$, Venekamp $\mathrm{N}$ et al. Exposure-response analyses of abiraterone and its metabolites in real-world patients with metastatic castration-resistant prostate cancer. Prostate Cancer Prostatic Dis 2019; 23: 244251.

5. Summary of Product Characteristics of abiraterone acetate. Available from: https://www.ema.europa.eu/en/documents/product-information/zytiga-epar-productinformation_en.pdf.

6. Chi KN, Spratlin J, Kollmannsberger C, North S, Pankras C, Gonzalez M et al. Food effects on abiraterone pharmacokinetics in healthy subjects and patients with metastatic castrationresistant prostate cancer. J Clin Pharmacol 2015; 55: 1406-1414.

7. Groenland SL, van Eerden RAG, Verheijen RB, Koolen SLW, Moes DJAR, Desar IME et al. Therapeutic drug monitoring of oral anticancer drugs: the DPOG-TDM protocol for a prospective study. Ther Drug Monit 2019; 41: 561-567.

8. Scher HI, Halabi S, Tannock I, Morris M, Sternberg CN, Carducci MA et al. Design and end points of clinical trials for patients with progressive prostate cancer and castrate levels of testosterone: recommendations of the Prostate Cancer Clinical Trials Working Group. J Clin Oncol 2008; 26: 1148-1159.

9. Scher HI, Morris MJ, Stadler WM, Higano C, Basch E, Fizazi K et al. Trial design and objectives for castration-resistant prostate cancer: updated recommendations from the Prostate Cancer Clinical Trials Working Group 3. J Clin Oncol 2016; 34: 1402-1418.

10. van Nuland $M$, Venekamp N, de Vries N, de Jong KAM, Rosing H, Beijnen JH. Development and validation of an UPLC-MS / MS method for the therapeutic drug monitoring of oral antihormonal drugs in oncology. J Chromatogr B 2019; 1106-1107: 26-34.

11. Food and Drug Administration. Abiraterone Clinical Pharmacology and Biopharmaceutics Review. 2010. Available from: https://www.accessdata.fda.gov/drugsatfda_docs/nda/2011/ 2023790rig1 s000ClinPharmR.pdf.

12. R Core Development Team. A language and environment for statistical computing. R Found Statistical Computing, Vienna. 2016. Available from: https://www.r-project.org/.

13. Szmulewitz RZ, Peer C, Ibraheem A, Martinez E, Kozloff MF, Carthon B et al. Prospective international randomized phase II study of low-dose abiraterone with food versus standard dose abiraterone in castration-resistant prostate cancer. J Clin Oncol 2018; 36: 1389-1395. 


\section{SUPPLEMENTARY DATA}

ABI \#1

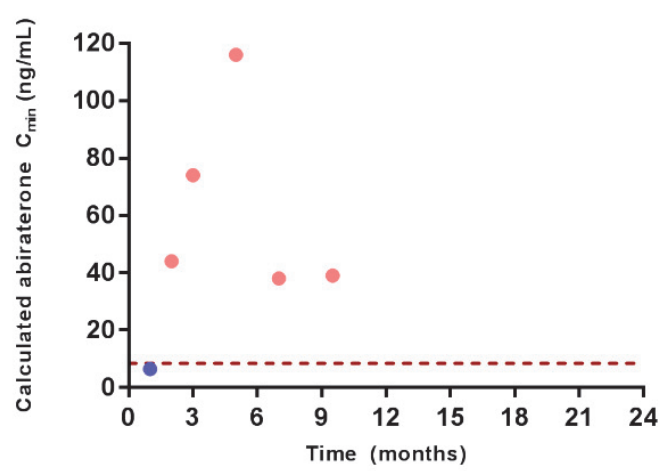

ABI \#6

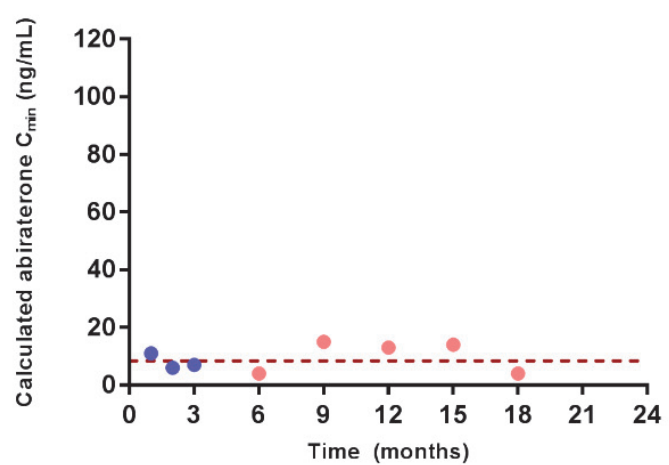

ABI \#9

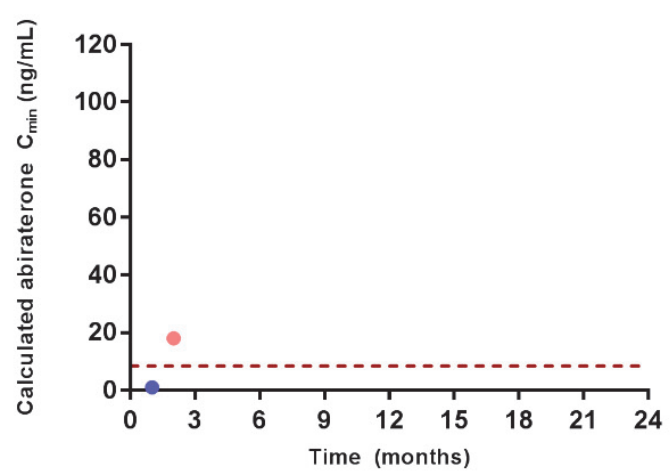

ABI \#5

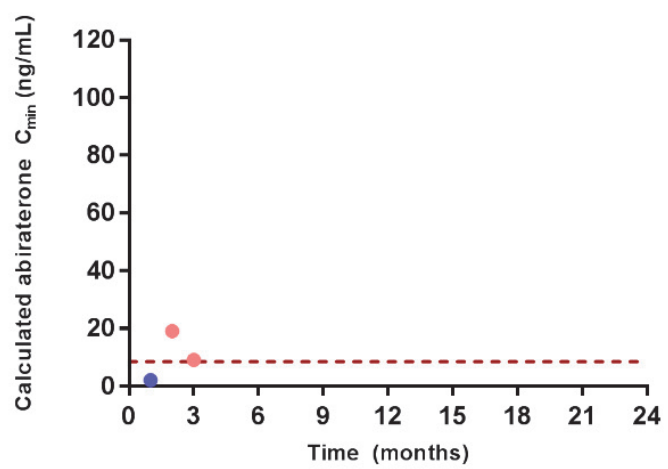

ABI \#7 (ongoing)

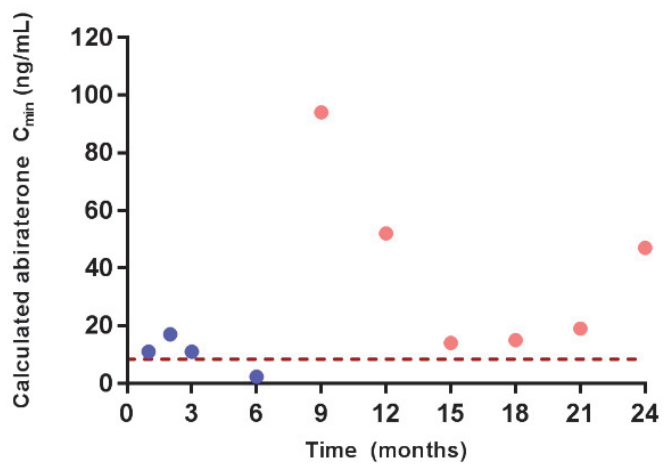

$\mathrm{ABI} \# 10$

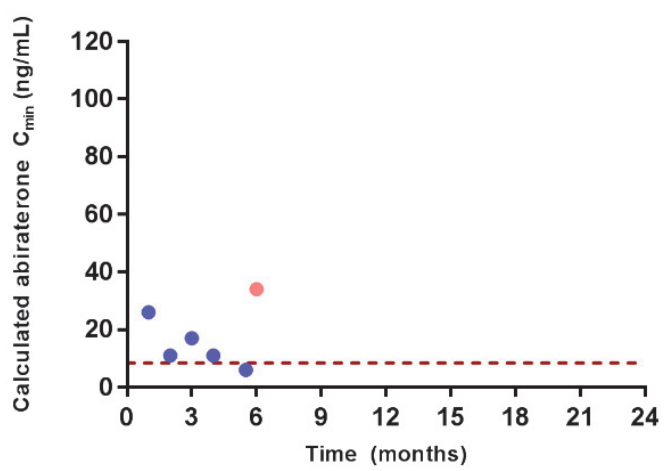

- abiraterone without food

- abiraterone with food 
ABI \#12

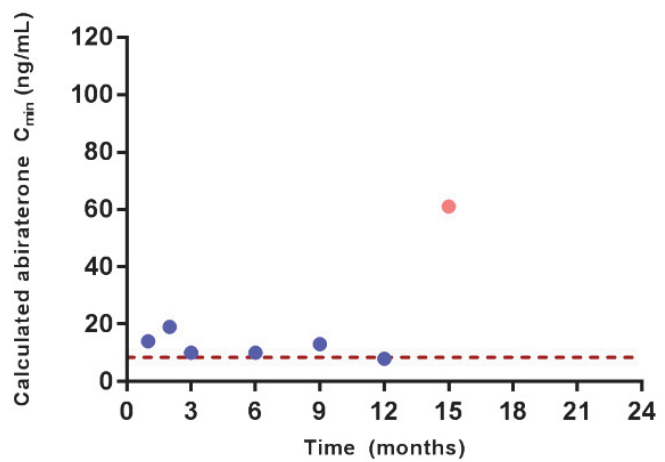

$A B I \# 14$ (ongoing)

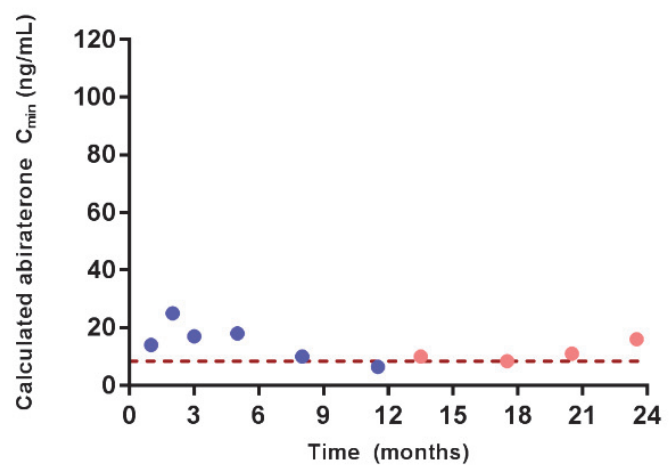

ABI \#16 (ongoing)

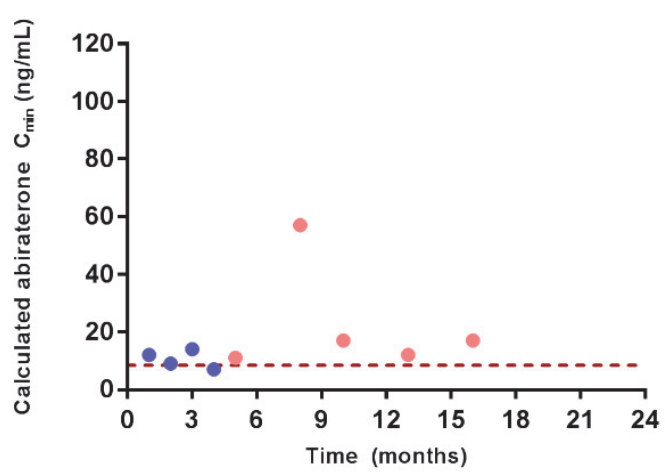

ABI \#13

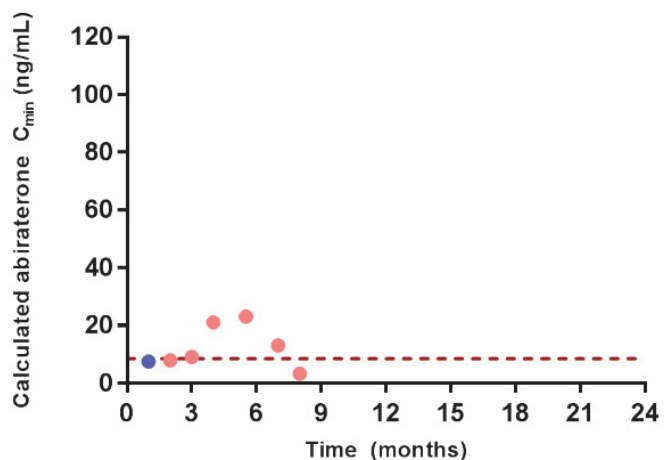

ABI \#15

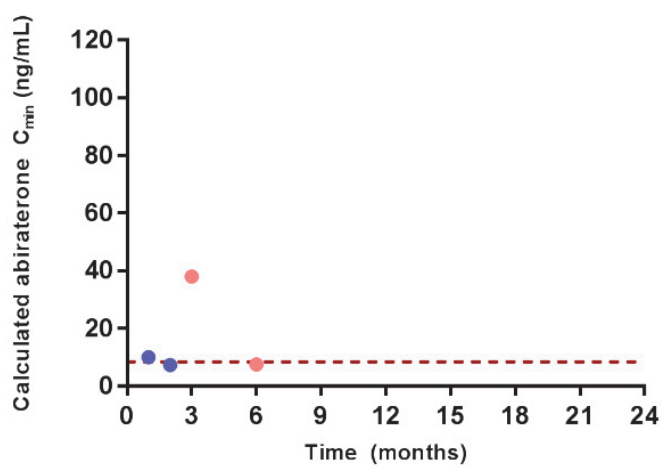

$A B I$ \#17 (ongoing)

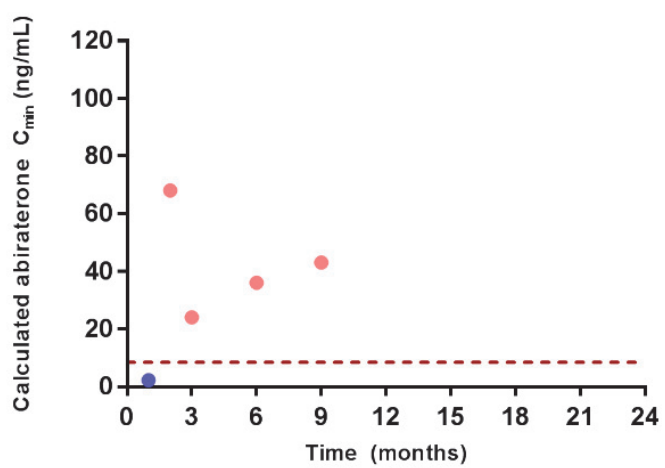

- abiraterone without food

- abiraterone with food 
ABI \#18

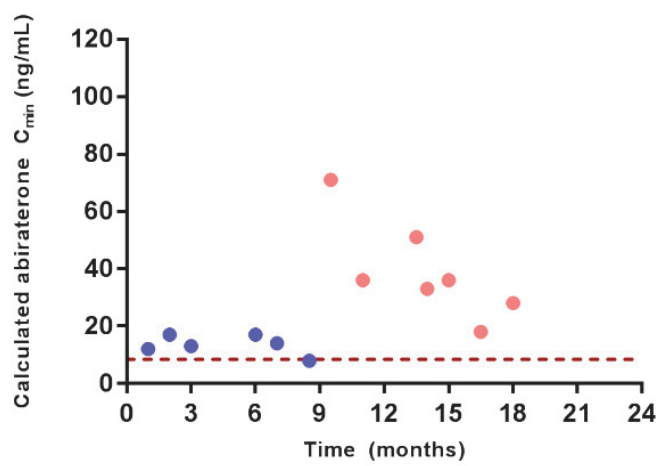

ABI \#29

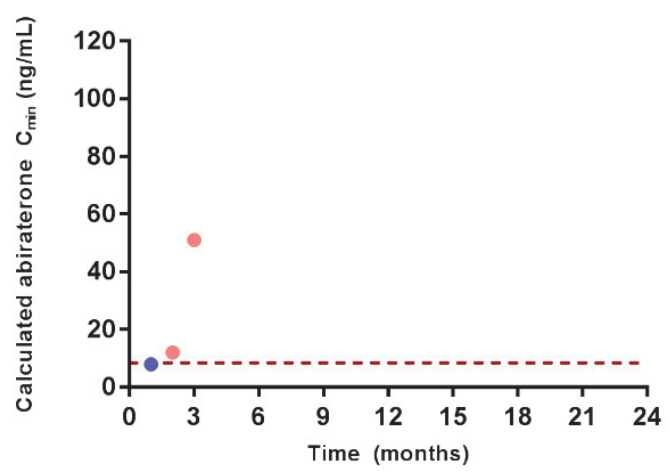

ABI \#35 (ongoing)

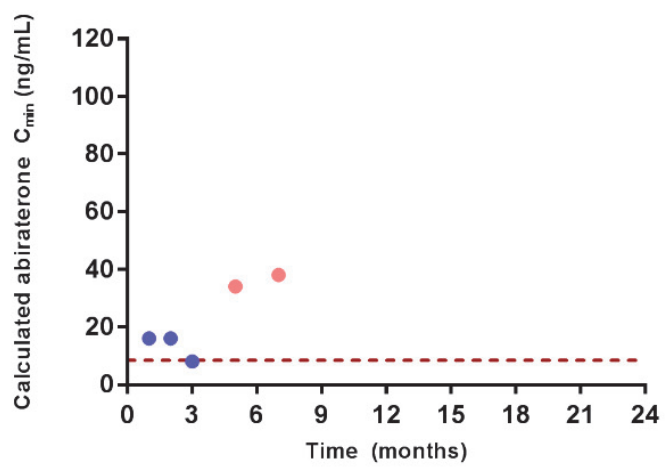

ABI \#22

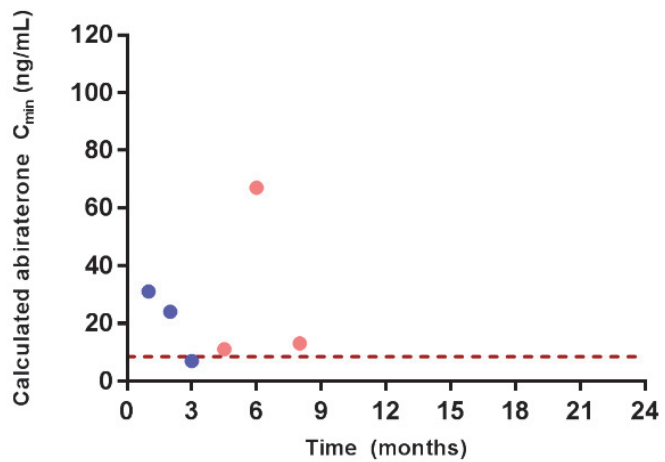

ABI \#34 (ongoing)

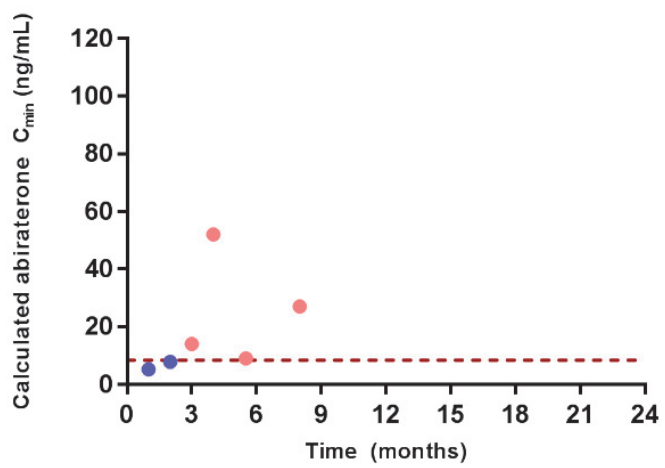

- abiraterone without food

- abiraterone with food

Supplementary Figure 1 - Individual patient graphs of the patients who received a food intervention and in whom the effect was evaluated $(n=17)$, depicting all calculated abiraterone $\mathrm{C}_{\min }$, before and after concomitant intake with a light meal or a snack.

The dashed line indicates the pharmacokinetic target of $C_{\min } \geq 8.4 \mathrm{ng} / \mathrm{mL}$. Blue dots indicate calculated abiraterone $C_{\min }$ in modified fasting state. Pink dots indicate calculated abiraterone $\mathrm{C}_{\min }$ concomitant with a light meal or a snack.

$C_{\text {min }}=$ minimum plasma concentration 



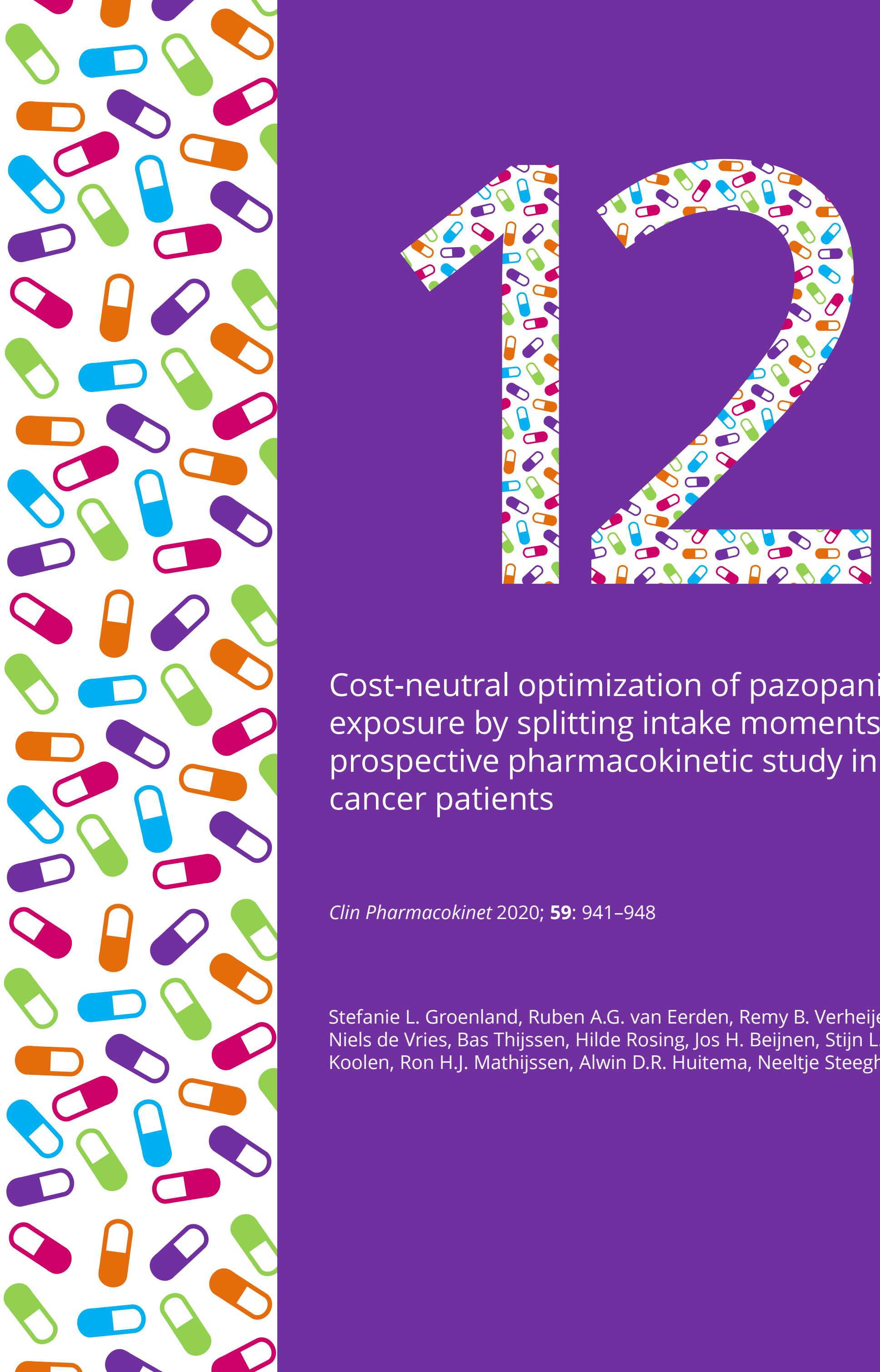

Cost-neutral optimization of pazopanib exposure by splitting intake moments: a prospective pharmacokinetic study in cancer patients

Clin Pharmacokinet 2020; 59: 941-948

Stefanie L. Groenland, Ruben A.G. van Eerden, Remy B. Verheijen, Niels de Vries, Bas Thijssen, Hilde Rosing, Jos H. Beijnen, Stijn L.W. Koolen, Ron H.J. Mathijssen, Alwin D.R. Huitema, Neeltje Steeghs 


\section{ABSTRACT}

\section{Background and objective}

Pazopanib is an oral tyrosine kinase inhibitor used in the treatment of renal cell carcinoma and soft-tissue sarcoma. At the approved dose of $800 \mathrm{mg}$ once daily (QD), $16-20 \%$ of patients are being underdosed and at risk of decreased efficacy. This study aimed to show whether splitting intake moments, as a cost-neutral alternative to a dose increase, leads to an increased exposure.

\section{Methods}

We performed a cross-over trial comparing the pharmacokinetics of pazopanib $800 \mathrm{mg}$ QD with pazopanib $400 \mathrm{mg}$ twice daily. Pharmacokinetic sampling was performed at steady-state for both dosing schedules.

\section{Results}

Nine evaluable patients were included. At the $800 \mathrm{mg}$ QD dosing schedule, median minimum plasma concentration $\left(C_{\min }\right)$, area under the concentration-time curve from zero to $24 \mathrm{~h}\left(\mathrm{AUC}_{0-24 \mathrm{~h}}\right)$, and maximum plasma concentration $\left(\mathrm{C}_{\max }\right)$ were $23.2 \mathrm{mg} / \mathrm{L}$ (interquartile range 18.5-27.6), $773 \mathrm{mg} \mathrm{h/L} \mathrm{(557-1009)} \mathrm{and} 40.6 \mathrm{mg} / \mathrm{L}$ (36.4-56.4) compared with 41.6 $\mathrm{mg} / \mathrm{L}$ (30.5-55.8, p=0.004), $942 \mathrm{mg} \mathrm{h/L} \mathrm{(885-1419,} \mathrm{p=0.027)} \mathrm{and} 50.2 \mathrm{mg} / \mathrm{L}$ (46.8-72.5, $\mathrm{p}=0.074$ ) at $400 \mathrm{mg}$ twice daily. One patient experienced a grade 3 event (i.e. diarrhea).

\section{Conclusions}

This study demonstrates that splitting intake moments of pazopanib leads to a $79 \%$ increase in $C_{\min }$, with acceptable tolerability. Therefore, this new dosing schedule offers a cost-neutral opportunity to optimize treatment in patients with low exposure.

\section{Clinical Trial Registration}

NL6137 (https://www.trialregister.nl) 


\section{INTRODUCTION}

Pazopanib is an oral tyrosine kinase inhibitor approved for the treatment of advanced renal cell carcinoma (RCC) and soft-tissue sarcoma (STS). Pazopanib is targeted at the vascular endothelial growth factor receptors-1, -2 and -3 , platelet-derived growth factor receptors- $\alpha$ and $-\beta$, fibroblast growth factor receptor, and stem cell factor receptor (c-Kit). ${ }^{1}$ In the phase III trial in patients with RCC, pazopanib prolonged progression-free survival from 4.2 to 9.2 months compared to placebo. ${ }^{2}$

Exposure-response analyses by Suttle et al. revealed that patients with RCC with a minimum plasma concentration $\left(C_{\min }\right) \geq 20.5 \mathrm{mg} / \mathrm{L}$ had a significantly longer progressionfree survival than patients with a $C_{\min }$ below this threshold (19.6 vs. 52.0 weeks, $\left.p=0.004\right){ }^{3}$ This exposure-efficacy threshold has been confirmed in the adjuvant setting and in a reallife patient cohort of patients with RCC. ${ }^{4,5}$ A similar trend was found for patients with STS as well, although not statistically significant. ${ }^{5}$

At the currently used fixed dose of $800 \mathrm{mg}$ once daily (QD), interindividual variability in pharmacokinetic exposure is high (40-70\% $)^{5-7}$ and about $16-20 \%$ of patients do not reach the efficacy threshold of $C_{\min } \geq 20.5 \mathrm{mg} / \mathrm{L}$. 3,5 These patients are thus underdosed and potentially at risk of decreased antitumor efficacy. This provides a strong rationale for therapeutic drug monitoring, which is individualized dosing based on measured drug concentrations. ${ }^{8}$ In a previous prospective clinical trial $(n=30)$ by Verheijen et al., it has been demonstrated that pharmacokinetically guided pazopanib dosing is feasible and results in an increased proportion of patients with adequate pharmacokinetic exposure. To achieve this, pazopanib dosages needed to be increased up to $1800 \mathrm{mg}$ QD in some cases. ${ }^{9}$ However, because of the non-linear absorption of pazopanib, which is plateauing at dosages above $800 \mathrm{mg}$, absolute dose increments are not an efficient strategy to increase pharmacokinetic exposure for pazopanib. ${ }^{10}$ Furthermore, it leads to an increase in treatment costs. Previously, we have developed a population pharmacokinetic model based on three clinical trials $(n=96)$ and have shown that the relative bioavailability of pazopanib dosed at $400 \mathrm{mg}$ is estimated to be $59 \%$ higher than at $800 \mathrm{mg} .{ }^{10}$ Therefore, we hypothesized that splitting intake moments would be a convenient and cost-neutral option for dose optimization.

The aim of this pharmacokinetic cross-over trial was to demonstrate whether switching patients from an $800 \mathrm{mg}$ QD to a $400 \mathrm{mg}$ twice daily (BID) dosing schedule leads to a significant increase in pharmacokinetic exposure, in particular $C_{\min }$. 


\section{METHODS}

\section{Study design}

We performed a prospective multi-center clinical trial with a cross-over design. Figure 1 provides a schematic overview of the study design. First, pharmacokinetic exposure was determined at the $800 \mathrm{mg}$ QD dosing schedule. Subsequently, patients switched to a 400 mg BID schedule for 7 days, after which the pharmacokinetic exposure was determined again at this new dosing schedule. As the elimination half-life $\left(t_{1 / 2}\right)$ of pazopanib is $31 \mathrm{~h}, 7$ days was accepted to be sufficient to attain steady-state concentrations at the $400 \mathrm{mg}$ BID dosing schedule (i.e. more than four to five times $t_{1 / 2}$ ). Patients were instructed to take pazopanib at approximately 8.00 a.m., and 8.00 p.m. at the BID dosing schedule, in a modified fasting state, meaning no food $2 \mathrm{~h}$ before and $1 \mathrm{~h}$ after drug intake. Patients requiring a dose interruption or dose reduction or who discontinued treatment during the study were considered non-evaluable for pharmacokinetic analysis and were replaced. At the end of the trial, pazopanib treatment was continued as part of standard care.

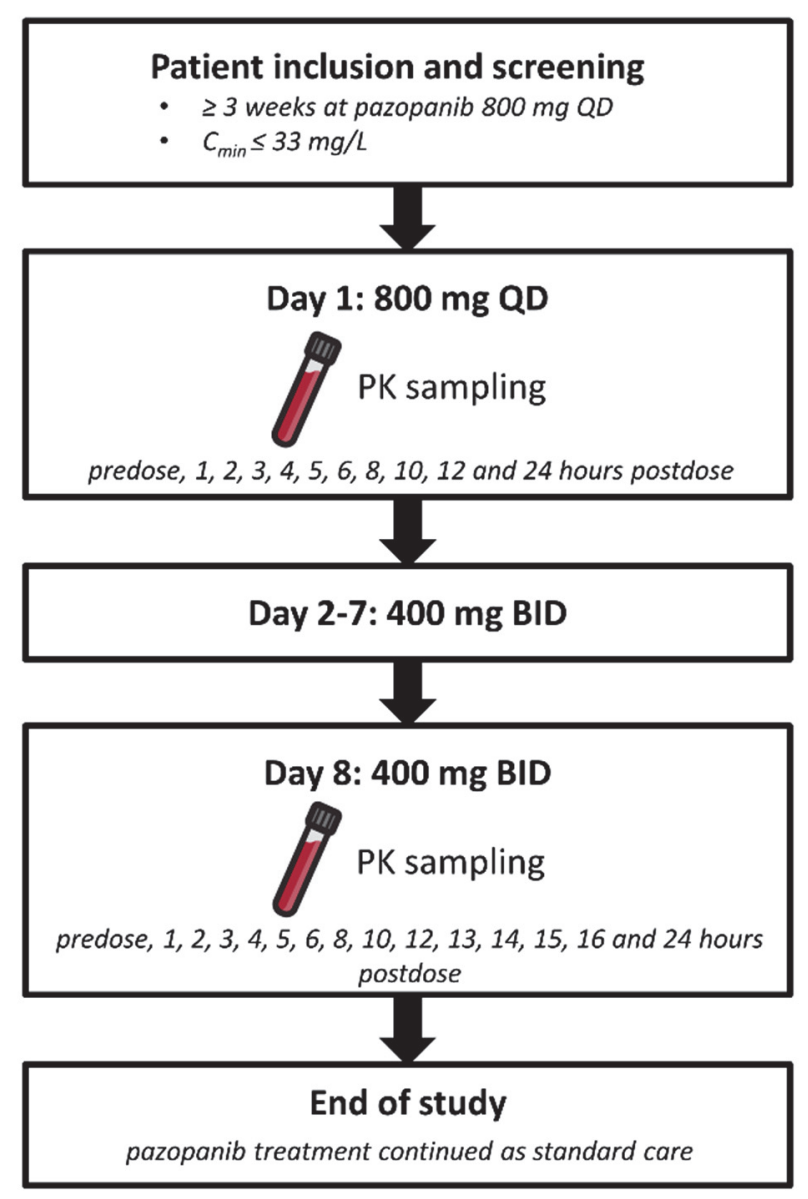

Figure 1 - Schematic overview of clinical trial design

At the $400 \mathrm{mg}$ BID dosing schedule, the second dose of pazopanib was taken 12 hours after the first dose. Sampling time points are relative to the first dose.

$B I D=$ twice daily; $C_{\min }=$ minimum plasma concentration; $P K=$ pharmacokinetic; $Q D=$ once daily 


\section{Patient population}

Patients with histological or cytological proof of cancer with an indication for treatment with pazopanib (i.e. advanced RCC or STS) were eligible for inclusion. Since evidence suggests pazopanib exposure may drop during the first weeks of treatment, all patients needed to be on pazopanib $800 \mathrm{mg}$ QD treatment $\geq 3$ weeks prior to start of the study. ${ }^{10}$ Further inclusion criteria were age $\geq 18$ years, World Health Organization performance status of 0,1 or 2 , and adequate organ function per judgement of the treating physician.

Patients were excluded in case of a (calculated) $C_{\min }>33 \mathrm{mg} / \mathrm{L}$ at screening, as by expecting an increase in $\mathrm{C}_{\min }$ of at least $50 \%$ after splitting intake moments based on previous simulations ${ }^{10}, C_{\min }$ is expected to rise above $50 \mathrm{mg} / \mathrm{L}$ in these patients, which is associated with an increased risk of toxicity. ${ }^{9}$ Another exclusion criterion was concomitant use of medication that could influence the pharmacokinetics of pazopanib within 14 days or five half-lives of the drug (whichever was shorter) before the start of the study, consisting of (but not limited to) gastric acid suppressing agents, cytochrome P450 3A4inhibitors/inducers, P-glycoprotein, and/or breast cancer resistance protein modulators.

\section{Pharmacokinetics}

At screening, either an actual trough concentration was drawn or $\mathrm{C}_{\min }$ was calculated using the following formula ${ }^{11}$ :

$$
C_{\text {min }}=C_{\text {measured }} * 0.5 \frac{\text { dosing interval-TAD }}{t_{1 / 2}}
$$

where $C_{\min }$ is the calculated minimum plasma concentration, $C_{\text {measured }}$ is the measured plasma concentration, dosing interval is the time between two consecutive administrations of the drug (i.e. $24 \mathrm{~h}$ for pazopanib), TAD is the time after dose (i.e. the time between the last drug intake and collection of the pharmacokinetic sample), and $t_{1 / 2}$ is the average elimination half-life of the drug (i.e. $31 \mathrm{~h}$ for pazopanib ${ }^{1}$ ).

At day 1 and day 8 of the study, patients were admitted to the hospital and blood samples were collected for pharmacokinetic analysis. Time points at day 1 (800 mg QD) were predose and $1,2,3,4,5,6,8,10,12$, and $24 \mathrm{~h}$ post-dose. Time points at day 8 (400 mg BID) were pre-dose and $1,2,3,4,5,6,8,10,12,13,14,15,16$, and 24 h post-dose. Sampling time points were relative to the first dose, the second dose was taken after collection of the $12 \mathrm{~h}$ post-dose sample. At each time point, blood samples were collected in 3-mL $\mathrm{K}_{2}$ EDTA tubes and centrifuged directly after collection (1500G, 5 minutes, $4^{\circ} \mathrm{C}$ ). Plasma was stored at $-20^{\circ} \mathrm{C}$ until analysis. Plasma pazopanib concentrations were measured using a validated liquid chromatography-tandem mass spectrometry method. ${ }^{12}$ 


\section{Study endpoint}

The primary endpoint of this study was to evaluate whether switching patients from an $800 \mathrm{mg}$ QD to a $400 \mathrm{mg}$ BID dosing schedule would lead to an increase in pharmacokinetic exposure, measured as $C_{\min }$ and area under the concentration-time curve from zero to 24 $\mathrm{h}\left(\mathrm{AUC}_{0-24 \mathrm{~h}}\right)$. The secondary endpoint was to compare adverse events between the two dosing schedules. As an exploratory endpoint, the cost effectiveness of this intervention compared with QD dose increments was evaluated.

\section{Safety assessments}

Recording of adverse events (AEs), vital signs, and hematology and blood chemistry assessments was performed at day 1 and day 8 of the study. The incidence, severity, and start and end dates of all AEs were recorded and graded according to the Common Terminology Criteria for Adverse Events, version 4.03. Toxicity at the $800 \mathrm{mg}$ QD dosing schedule was assessed at screening and at day 1. Only toxicities that were present at that time, were taken into account. Toxicity at the $400 \mathrm{mg}$ BID dosing schedule was assessed at day 8.

\section{Statistics}

Splitting intake moments was considered to result in an increase in $\mathrm{C}_{\min }$ and $\mathrm{AUC}_{0-24 \mathrm{~h}}$ of at least $50 \%$, based on previous simulations. ${ }^{10}$ By assuming an intra-individual standard deviation of the difference between the two dosing schedules of $50 \%$, ten evaluable patients had to be included to obtain $80 \%$ power (two-sided $a=0.05$ ) to detect this increase of $\geq 50 \%$. Pharmacokinetic parameters were calculated using non-compartmental analysis. $C_{\min }$ was defined as the median value of the pre-dose and $24 \mathrm{~h}$ post-dose sample for the $800 \mathrm{mg}$ QD dosing schedule, and of the pre-dose, 12 and $24 \mathrm{~h}$ post-dose sample for the $400 \mathrm{mg}$ BID dosing schedule. $\mathrm{AUC}_{0-24 \mathrm{~h}}$ was calculated using the linear/log trapezoidal method. $C_{\max }$ was defined as the highest measured concentration for each dosing schedule. Minimum plasma concentration, $A \cup C_{0-24 h}$, and $C_{\max }$ of the two dosing schedules were compared using two-sided Wilcoxon signed rank tests. All statistical analyses were performed using R version 3.3.2 (R Project, Vienna, Austria). ${ }^{13}$

\section{Ethics approval and consent to participate}

The study was approved by the Medical Ethics Committee of The Netherlands Cancer Institute-Antoni van Leeuwenhoek. Participating centers were The Netherlands Cancer Institute-Antoni van Leeuwenhoek and the Erasmus MC Cancer Institute. Local approval was obtained in each participating center. The study was conducted in accordance with Good Clinical Practice and the Declaration of Helsinki. All patients provided written informed consent prior to enrollment in the study. This trial was registered in the Netherlands Trial Register (https://www.trialregister.nl, NL6137) and the EudraCT 
database (2016-005252-21). The full trial protocol can be accessed upon reasonable request by contacting the corresponding author.

\section{RESULTS}

\section{Patient characteristics}

In total, 11 patients were enrolled in the study from June 2017 until January 2019, of which nine patients were evaluable for pharmacokinetic analyses. In one patient, pazopanib treatment was interrupted because of toxicity before all pharmacokinetic measurements were completed and one patient did not take pazopanib according to the protocol. Both of these patients were excluded. Initially, unevaluable patients were replaced according to the protocol. However, the last patient was unevaluable after the study had been closed. As the study was already positive on its primary endpoint of change in $\mathrm{C}_{\text {min, }}$ it was decided not to replace this patient. Baseline characteristics of all patients are provided in Table 1. The majority of patients were female (73\%), and the median age was 61 years. Six patients were diagnosed with RCC and five patients with STS. Median time on pazopanib treatment before enrollment in the study was 4.5 months.

Table 1 - Baseline characteristics of all patients $(n=11)$ and evaluable patients $(n=9)$

\begin{tabular}{|c|c|c|}
\hline Characteristic & All patients $(n=11)$ & Evaluable patients $(\mathrm{n}=9)$ \\
\hline Gender, female & $8(73 \%)$ & $7(78 \%)$ \\
\hline Age (years) & $61[42-78]$ & $55[42-78]$ \\
\hline \multicolumn{3}{|l|}{ Tumor type } \\
\hline Renal cell carcinoma & $6(55 \%)$ & $6(67 \%)$ \\
\hline Soft tissue sarcoma & $5(45 \%)$ & $3(33 \%)$ \\
\hline \multicolumn{3}{|l|}{ WHO performance status } \\
\hline 0 & $6(55 \%)$ & $5(56 \%)$ \\
\hline 1 & $4(36 \%)$ & $4(44 \%)$ \\
\hline 2 & $1(9 \%)$ & $0(0 \%)$ \\
\hline $\begin{array}{l}\text { Previous lines of systemic } \\
\text { treatment (number) }\end{array}$ & $0[0-2]$ & $0[0-2]$ \\
\hline \multicolumn{3}{|l|}{ Previous systemic treatment } \\
\hline Chemotherapy & $4(36 \%)$ & $3(33 \%)$ \\
\hline Targeted therapy & $1(9 \%)$ & $0(0 \%)$ \\
\hline Time on pazopanib (months) & $4.5[0.7-28.7]$ & $4.5[0.7-23.7]$ \\
\hline
\end{tabular}

Data are expressed as no. (\%) or median [range], as appropriate

\section{Pharmacokinetics}

Figure 2 shows the pazopanib concentration-time curves at both dosing schedules. An overview of the pharmacokinetic parameters for each of the dosing schedules is provided in Table 2. In Figure 3, plots of $C_{\min }, A \cup C_{0-24 h}$, and $C_{\max }$ at both dosing schedules are shown. Using the $800 \mathrm{mg}$ QD dosing schedule, median $\mathrm{C}_{\min }, \mathrm{AUC}_{0-24 \mathrm{~h}}$, and $\mathrm{C}_{\max }$ were 23.2 
$\mathrm{mg} / \mathrm{L}$ (interquartile range (IQR) 18.5-27.6), $773 \mathrm{mg} \mathrm{h/L} \mathrm{(IQR} \mathrm{557-1009),} \mathrm{and} 40.6 \mathrm{mg} / \mathrm{L}$ (IQR 36.4-56.4), respectively. Switching to the $400 \mathrm{mg}$ BID dosing schedule resulted in an increase in $C_{\min }, A \cup C_{0-24 h}$, and $C_{\max }$ to $41.6 \mathrm{mg} / \mathrm{L}$ (IQR 30.5-55.8, 79\% increase, $p=0.004$ ), $942 \mathrm{mg} \mathrm{h} / \mathrm{L}$ (IQR 885-1419, 22\% increase, p=0.027), and $50.2 \mathrm{mg} / \mathrm{L}$ (IQR 46.8-72.5, 19\% increase, $p=0.074)$, respectively.

\section{Adverse events}

An overview of all treatment-related AEs is provided in Table 3. All but one patient experienced treatment-related AEs. No patients discontinued treatment and none required a dose reduction because of an adverse event. A single patient experienced a grade 3 event of diarrhea at the $400 \mathrm{mg}$ BID dosing schedule, for which pazopanib treatment was interrupted at day 6 of the study. Calculated $C_{\min }$ at this time was high (72.9 mg/L). Treatment was resumed after five days at $800 \mathrm{mg}$ QD, without toxicity. This patient was excluded from the pharmacokinetic analysis, because no PK samples were available at the $400 \mathrm{mg}$ BID dosing schedule.

Table 2 - Pharmacokinetic parameters of pazopanib at the $800 \mathrm{mg}$ QD and $400 \mathrm{mg}$ BID dosing schedule $(n=9)$

\begin{tabular}{lllll}
\hline PK parameter & $\mathbf{8 0 0} \mathbf{m g}$ QD & $\mathbf{4 0 0} \mathbf{~ m g ~ B I D ~}$ & $\begin{array}{l}\text { Percentage } \\
\text { change }\end{array}$ & p-value \\
\hline $\mathbf{C}_{\min }(\mathrm{mg} / \mathrm{L})^{\mathrm{a}}$ & $23.2(18.5-27.6)$ & $41.6(30.5-55.8)$ & $+79 \%$ & $\mathbf{0 . 0 0 4}$ \\
\hline $\mathbf{A U C}_{\mathbf{0}-24 \mathrm{~h}}\left(\mathrm{mg} \mathrm{h}^{\star \mathrm{h}} \mathrm{L}\right)^{\mathrm{b}}$ & $773(557-1009)$ & $942(885-1419)$ & $+22 \%$ & $\mathbf{0 . 0 2 7}$ \\
\hline $\mathbf{C}_{\max }(\mathrm{mg} / \mathrm{L})^{\mathrm{c}}$ & $40.6(36.4-56.4)$ & $50.2(46.8-72.5)$ & $+19 \%$ & 0.074 \\
\hline
\end{tabular}

Bold values indicate statistically significant $p$ values.

Data expressed as median (IQR).

${ }^{a} C_{\min }$ was defined as the median value of the predose and 24 hours postdose sample for the 800 $\mathrm{mg}$ QD dosing schedule, and of the predose, 12 and 24 hours postdose sample for the $400 \mathrm{mg}$ BID dosing schedule.

${ }^{\mathrm{b}} \mathrm{AUC} \mathrm{C}_{0-24 \mathrm{~h}}$ was calculated using the linear/log trapezoidal method.

${ }^{\mathrm{C}} \mathrm{C}_{\max }$ was defined as the highest measured concentration for each dosing schedule.

$A \cup C_{0-24 h}=$ area under the plasma concentration-time curve from time zero to 24 hours; $B I D=$ twice daily, $C_{\max }=$ maximum plasma concentration; $C_{\min }=$ minimum plasma concentration; $I Q R=$ interquartile range; $Q D=$ once daily 


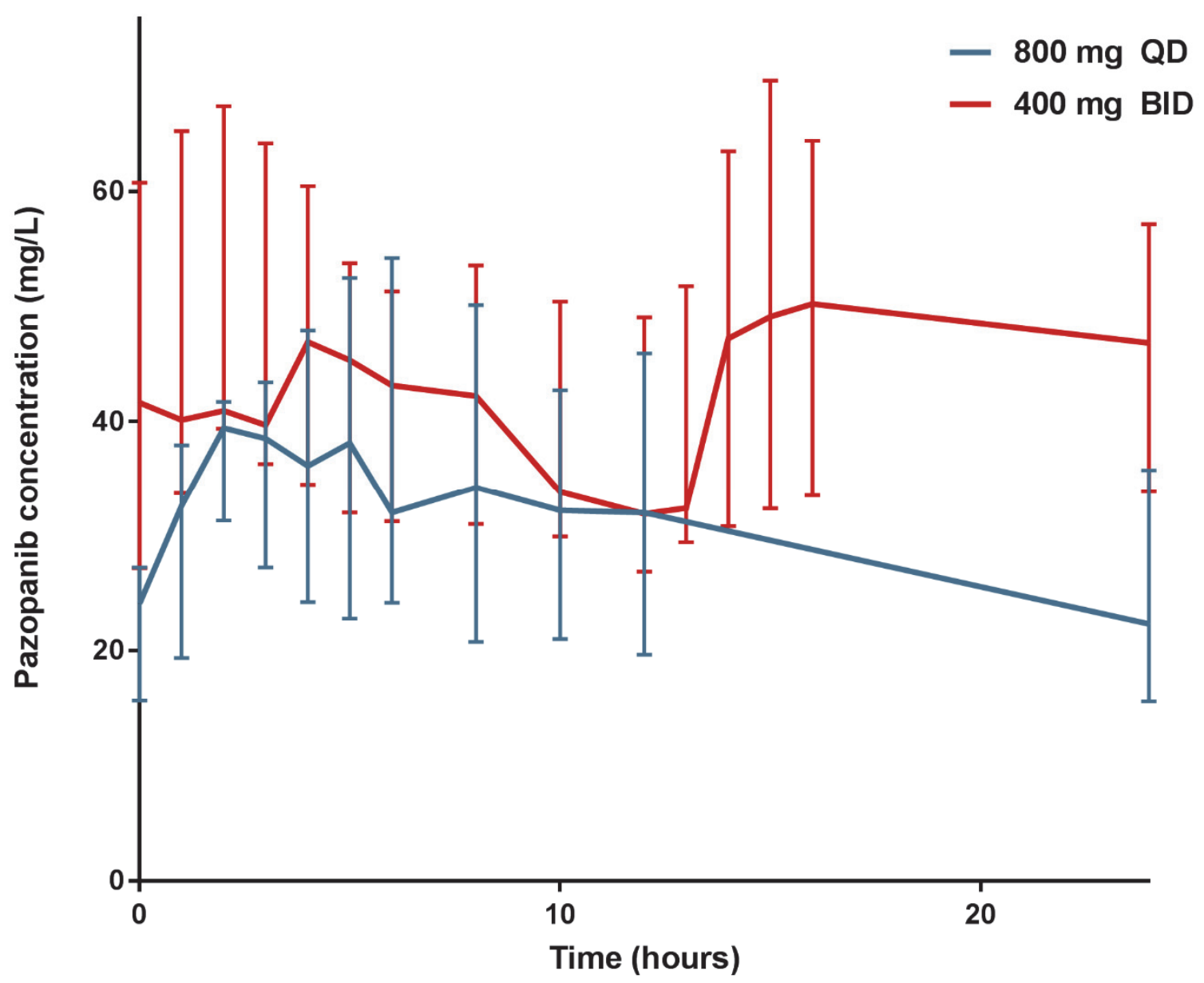

Figure 2 - Pazopanib plasma concentration-time curves (median + IQR) of the $800 \mathrm{mg}$ QD and $400 \mathrm{mg}$ BID dosing schedule ( $n=9)$
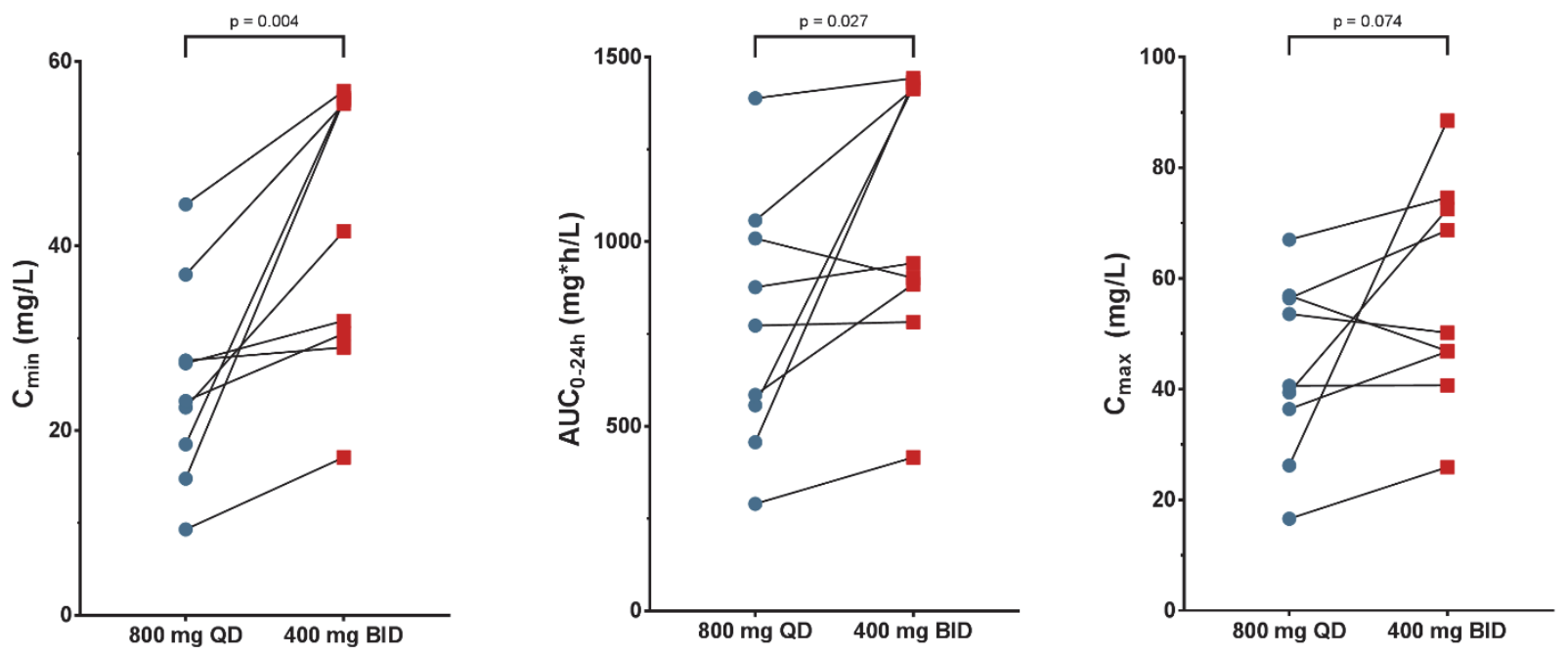

Figure 3 - Plots of pazopanib $C_{\min }, A_{U} C_{0-24 h}$ and $C_{\max }$ for both dosing schedules ( $\left.n=9\right)$

$C_{\min }$ was defined as the median value of the predose and 24 hours postdose sample for the 800 mg QD dosing schedule, and of the predose, 12 and 24 hours postdose sample for the $400 \mathrm{mg}$ BID dosing schedule. $A \cup C_{0-24 h}$ was calculated using the linear/log trapezoidal method. $C_{\text {max }}$ was defined as the highest measured concentration for each dosing schedule.

$A \cup C_{0-24 h}=$ area under the plasma concentration-time curve from time zero to 24 hours; $B I D=$ twice daily; $C_{\max }=$ maximum plasma concentration; $C_{\min }=$ minimum plasma concentration; $Q D=$ once daily 
Table 3 - Treatment-related adverse events (all patients, $n=11$ ), according to CTCAE v4.03

\begin{tabular}{|c|c|c|c|c|}
\hline \multirow[t]{2}{*}{ Adverse event } & \multicolumn{2}{|c|}{$800 \mathrm{mg}$ QD } & \multicolumn{2}{|c|}{$400 \mathrm{mg}$ BID } \\
\hline & Any grade $(\mathrm{n})$ & Grade $\geq 3(n)$ & Any grade $(n)$ & Grade $\geq 3$ (n) \\
\hline Diarrhea & 4 & 0 & 6 & 1 \\
\hline Fatigue & 5 & 0 & 8 & 0 \\
\hline Hypertension & 4 & 0 & 4 & 0 \\
\hline Nausea & 3 & 0 & 4 & 0 \\
\hline Hypothyroidism & 2 & 0 & 3 & 0 \\
\hline Anorexia & 2 & 0 & 3 & 0 \\
\hline Dysgeusia & 1 & 0 & 1 & 0 \\
\hline Vomiting & 0 & 0 & 2 & 0 \\
\hline Myalgia & 1 & 0 & 1 & 0 \\
\hline Thrombocytopenia & 1 & 0 & 1 & 0 \\
\hline Bilirubin increase & 1 & 0 & 1 & 0 \\
\hline Localized edema & 1 & 0 & 1 & 0 \\
\hline Hair change & 1 & 0 & 1 & 0 \\
\hline Skin hypopigmentation & 1 & 0 & 1 & 0 \\
\hline Abdominal pain & 1 & 0 & 1 & 0 \\
\hline Headache & 1 & 0 & 1 & 0 \\
\hline \multicolumn{5}{|l|}{ Generalized muscle } \\
\hline weakness & 1 & 0 & 1 & 0 \\
\hline Weight loss & 1 & 0 & 1 & 0 \\
\hline \multicolumn{5}{|l|}{ Total number of patients } \\
\hline experiencing AEs & 7 & 0 & 10 & 1 \\
\hline
\end{tabular}

$A E=$ adverse event; $B I D=$ twice daily; $C T C A E=$ Common Terminology Criteria for Adverse Events; $Q D=$ once daily

\section{DISCUSSION}

In this prospective, multi-centre, cross-over trial we evaluated the effect of splitting intake moments of pazopanib from $800 \mathrm{mg}$ QD into $400 \mathrm{mg}$ BID on the pharmacokinetic exposure. This intervention resulted in a significant increase in $C_{\min }$ and $A \cup C_{0-24 h}$ of $79 \%$ and $22 \%$, respectively, with acceptable tolerability (Figure 2, Figure 3, Table 2). $C_{\max }$ was also numerically higher (19\%), although this difference was not statistically significant. Thereby, splitting intake moments offers a convenient strategy to optimize pazopanib treatment for patients with a low pharmacokinetic exposure.

As the number of doses increases, $C_{\min }$ is expected to increase as well, even if the bioavailability would remain equal. However, the increased bioavailability is reflected by the significant increase in AUC of $22 \%$, which demonstrates the proof of principle of splitting intake moments for pazopanib. The efficacy threshold of $C_{\min } \geq 20.5 \mathrm{mg} / \mathrm{L}$ is determined for a once daily dosing schedule and reflects a certain total exposure (i.e. $A \cup C)$. As AUC does not increase to the same extent as $C_{\min }$ when splitting intake moments, 
the same $C_{\min }$ value at a twice daily dosing schedule reflects a lower total exposure. In general, if a certain total exposure is needed for efficacy, using the same $C_{\text {min }}$ target for a twice daily dosing schedule could result in a potential risk of underdosing. Therefore, for pazopanib it should be further investigated if the target of $C_{\min } \geq 20.5 \mathrm{mg} / \mathrm{L}$ also applies for the twice daily dosing schedule or that a higher threshold should be used. Since exposure-efficacy analyses were only performed for $\mathrm{C}_{\min }$ and not for AUC, it is unknown which parameter most accurately predicts clinical response. However, $C_{\min }$ is the most pragmatic predictor, as only a single plasma sample is needed.

Pazopanib shows a complex absorption profile, which has been described by Yu et al. and consists of a sequential fast and slow absorption phase. This is explained by the fact that pazopanib is only water soluble at $\mathrm{pH}<4$, resulting in a fast absorption at the first part of the intestine (i.e. the duodenum), when pazopanib is still in solution. As the pH rises sharply $>4$ in the small intestines, pazopanib precipitates (i.e. speculation based on the above mentioned physiological considerations ${ }^{14}$ ) and further absorption becomes dissolution rate limited, which occurs much slower. The poor solubility of pazopanib also explains the previously observed dose dependency in relative bioavailability, which was estimated to be $59 \%$ higher at $400 \mathrm{mg}$ compared with $800 \mathrm{mg} .{ }^{10}$

In fact, the oral bioavailability of pazopanib at the approved dose of $800 \mathrm{mg}$ is only 14$39 \%$, due to its suboptimal pharmaceutical formulation. ${ }^{15}$ This results in both a high interand intra-individual variability in pharmacokinetic exposure. In a previous study, we have shown that with an improved pharmaceutical formulation with a much better dissolution profile, only $300 \mathrm{mg}$ QD is needed to attain a similar pharmacokinetic exposure as with $800 \mathrm{mg}$ QD of the current formulation. ${ }^{16}$ However, this improved formulation is not available in clinical practice.

As the bioavailability increases by splitting intake moments of pazopanib, variability was expected to decline. ${ }^{17}$ However, in this study we still observed a substantial interindividual variability at $400 \mathrm{mg}$ BID. Hence, plasma concentrations and toxicity should still be carefully monitored. In addition, relatively large individual differences in increases were seen between patients (Figure 3), which could be explained by the relatively high intraindividual variability of pazopanib (i.e. $24.7 \%{ }^{18}$ ) and differences in drug absorption between patients.

In a previous pharmacokinetically guided dosing study of pazopanib, dose increments to dosages ranging from $1000 \mathrm{mg}$ QD to $1800 \mathrm{mg}$ QD were needed to attain adequate exposure. ${ }^{9}$ The costs of these additional $200-1000 \mathrm{mg}$ of pazopanib are $€ 873$ - $€ 4342$ per patient per month in The Netherlands, part of which could theoretically be saved when splitting intake moments is used to increase pharmacokinetic exposure. Because dose increments in the case of low exposure are currently not implemented as standard of care, this calculation does not represent an actual cost saving. Instead, it represents a 
comparison of the costs between absolute dose increments and splitting intake moments. As splitting intake moments does not lead to any additional costs compared to the standard dose of $800 \mathrm{mg} Q \mathrm{Q}$, it can be concluded that this is a cost-neutral strategy.

A strength of the current study is that full pharmacokinetic curves instead of only trough samples were obtained at both dosing schedules, enabling comparison of $\mathrm{C}_{\max }$ and $A \cup C_{0}$. $24 \mathrm{~h}$ as well. Limitations of this study include the fact that toxicity of the QD and BID schedule could not be reliably compared because of the short duration of BID dosing (i.e. only seven days) and the fact that only patients who already tolerated the $800 \mathrm{mg}$ QD dose for multiple weeks were eligible for enrollment. Although this was sufficient to reach steady-state concentrations, more time might be needed for adverse events to emerge. Furthermore, only nine evaluable patients were included instead of ten. A post hoc power calculation indicated that nine patients provided $75 \%$ power to detect an increase in pharmacokinetic exposure of $50 \%$.

A drawback of switching to a twice daily dosing schedule could be the inconvenience for patients with regard to the modified fasting state in which pazopanib should be administered. An alternative strategy to increase pharmacokinetic exposure to pazopanib could be concomitant intake with food. ${ }^{7,19}$ Both strategies could be applied to reach the predefined target. We are currently performing a prospective study on therapeutic drug monitoring of oral anticancer drugs, including pazopanib, in which we split intake moments as a first step to optimize pazopanib treatment in case of low pharmacokinetic exposure. As a second step, concomitant intake with food is recommended..$^{8,20}$

The current study demonstrated that previous simulations using a population pharmacokinetic model of pazopanib adequately predicted the effect of splitting intake moments on $\mathrm{C}_{\min }$ (i.e. $75 \%$ vs. $79 \%$ increase), validating a population pharmacokinetic simulation approach for changes in pazopanib dosing schedules. ${ }^{10}$ It has to be noted, though, that the increase in $\mathrm{AUC}_{0-24 \mathrm{~h}}$ was less pronounced than the simulations predicted (i.e. $22 \%$ vs. $59 \%$ ). However, pazopanib pharmacokinetics shows wide variability, and consequently, comparisons based on a relatively small sample size are difficult. Furthermore, this study illustrates the relevance of population pharmacokinetic simulations in general, which could be applied more often in oncology. These simulations could provide a rationale for proof-of-concept pharmacokinetic studies. The pharmacokinetic data of these clinical studies could then be added to the original population pharmacokinetic model, to further optimize its predictions.

Implications of this study for clinical practice are that patients with pazopanib $\mathrm{C}_{\min }<20.5$ $\mathrm{mg} / \mathrm{L}$ could be switched from an $800 \mathrm{mg}$ QD to a $400 \mathrm{mg}$ BID dosing schedule to improve pharmacokinetic exposure. The feasibility, tolerability, and efficacy of this strategy will now be further studied in a prospective clinical study on therapeutic drug monitoring of oral anticancer drugs (https://www.trialregister.nl; NL6695). ${ }^{8}$ Furthermore, data of the 
current study could be added to the existing population pharmacokinetic model to better characterize the non-linear pharmacokinetics of pazopanib and to further optimize the dosing schedule.

\section{CONCLUSIONS}

This study demonstrates that pharmacokinetic exposure to pazopanib can be boosted by splitting intake moments from $800 \mathrm{mg}$ QD to $400 \mathrm{mg}$ BID, leading to a significant increase in $C_{\min }$ of $79 \%$ with acceptable tolerability. This is relevant for the $16-20 \%$ of patients who are currently underdosed and therefore have a risk of decreased efficacy. As the observed variability in pazopanib pharmacokinetics is large, also after splitting the dose, this strategy should only be applied for those patients where follow-up blood concentration monitoring is in place. Hence, splitting intake moments offers a simple, effective, and costneutral strategy to optimize treatment in the significant subset of patients with a low pazopanib exposure.

\section{ACKNOWLEDGEMENTS}

We thank all patients for their participation in this study. We thank dr. Huixin Yu for performing the simulations. In addition, we thank the study team for their contribution, in particular Else Meijer and Brigitte Dufourny. This work was presented in part at the 2019 American Society of Clinical Oncology Annual Meeting, Chicago, IL, USA (J Clin Oncol. 2019; 37 Suppl.: abstract 3119). 


\section{REFERENCES}

1. Summary of Product Characteristics of pazopanib. Available from: http://www.ema.europa.eu/docs/nl_NL/document_library/EPAR_Product_Information/hu man/001141/WC500094272.pdf.

2. Sternberg CN, Davis ID, Mardiak J, Szczylik C, Lee E, Wagstaff J et al. Pazopanib in locally advanced or metastatic renal cell carcinoma: results of a randomized phase III trial. J Clin Oncol 2010; 28: 1061-1068.

3. Suttle AB, Ball HA, Molimard M, Hutson TE, Carpenter C, Rajagopalan D et al. Relationships between pazopanib exposure and clinical safety and efficacy in patients with advanced renal cell carcinoma. Br J Cancer 2014; 111: 1-8.

4. Sternberg CN, Donskov F, Haas NB, Doehn C, Russo P, Elmeliegy M et al. Pazopanib exposure relationship with clinical efficacy and safety in the adjuvant treatment of advanced renal cell carcinoma. Clin Cancer Res 2018; 24: 3005-3013.

5. Verheijen RB, Swart LE, Beijnen JH, Schellens JHM, Huitema ADR, Steeghs N. Exposuresurvival analyses of pazopanib in renal cell carcinoma and soft tissue sarcoma patients: opportunities for dose optimization. Cancer Chemother Pharmacol 2017; 80: 1171-1178.

6. Hurwitz HI, Dowlati A, Saini S, Savage S, Suttle AB, Gibson DM et al. Phase I trial of pazopanib in patients with advanced cancer. Clin Cancer Res 2009; 15: 4220-4227.

7. Lubberman FJE, Gelderblom H, Hamberg P, Vervenne WL, Mulder SF, Jansman FGA et al. The effect of using pazopanib with food vs fasted on pharmacokinetics, patient safety and preference (DIET study). Clin Pharmacol Ther 2019; 106: 1076-1082.

8. Groenland SL, van Eerden RAG, Verheijen RB, Koolen SLW, Moes DJAR, Desar IME et al. Therapeutic drug monitoring of oral anticancer drugs: the DPOG-TDM protocol for a prospective study. Ther Drug Monit 2019; 41: 561-567.

9. Verheijen RB, Bins S, Mathijssen RHJ, Lolkema MP, van Doorn L, Schellens JHM et al. Individualized pazopanib dosing: a prospective feasibility study in cancer patients. Clin Cancer Res 2016; 22: 5738-5746.

10. Yu H, van Erp N, Bins S, Mathijssen RHJ, Schellens JHM, Beijnen JH et al. Development of a pharmacokinetic model to describe the complex pharmacokinetics of pazopanib in cancer patients. Clin Pharmacokinet 2017; 56: 293-303.

11. Wang $Y$, Chia Y, Nedelman J, Schran H, Mahon F, Molimard M. A therapeutic drug monitoring algorithm for refining the imatinib trough level obtained at different sampling times. Ther Drug Monit 2009; 31: 579-584.

12. Herbrink M, de Vries N, Rosing H, Huitema ADR, Nuijen B, Schellens JHM et al. Quantification of 11 therapeutic kinase inhibitors in human plasma for therapeutic drug monitoring using liquid chromatography coupled with tandem mass spectrometry. Ther Drug Monit 2016; 38: 649-656.

13. R Core Development Team. A language and environment for statistical computing. $R$ Foundation for Statistical Computing, Vienna, Austria. 2019. Available from: https://www.rproject.org/.

14. van Leeuwen RW, van Gelder T, Mathijssen RHJ, Jansman FG. Drug-drug interactions with tyrosine-kinase inhibitors: a clinical perspective. Lancet Oncol 2014; 15: e315-e326. 
15. Deng Y, Sychterz C, Suttle AB, Dar MM, Bershas D, Negash K et al. Bioavailability, metabolism and disposition of oral pazopanib in patients with advanced cancer. Xenobiotica 2013; 43: 443-453.

16. Herbrink M, Groenland SL, Huitema ADR, Schellens JHM, Beijnen JH, Steeghs $\mathrm{N}$ et al. Solubility and bioavailability improvement of pazopanib hydrochloride. Int J Pharm 2018; 544: 181-190.

17. Hellriegel ET, Bjornsson TD, Hauck WW. Interpatient variability in bioavailability is related to the extent of absorption: implications for bioavailability and bioequivalence studies. Clin Pharmacol Ther 1996; 60: 601-607.

18. de Wit D, van Erp NP, den Hartigh J, Wolterbeek R, Deursen MDH, Labots M et al. Therapeutic drug monitoring to individualize the dosing of pazopanib: a pharmacokinetic feasibility study. Ther Drug Monit 2015; 37: 331-338.

19. Heath El, Chiorean EG, Sweeney CJ, Hodge JP, Lager JJ, Forman K et al. A phase I study of the pharmacokinetic and safety profiles of oral pazopanib with a high-fat or low-fat meal in patients with advanced solid tumors. Clin Pharmacol Ther 2010; 88: 818-823.

20. Groenland SL, van Eerden RAG, Koolen SLW, Moes DJAR, Desar IME, Touw D et al. Therapeutic drug monitoring of oral anticancer drugs - preliminary results of a prospective study. Ann Oncol 2019; 30: v159-v193. 


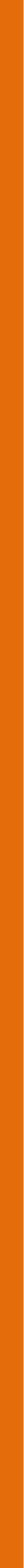




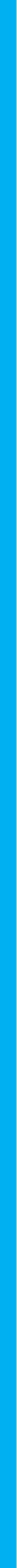




\section{ABSTRACT}

Palbociclib, ribociclib and abemaciclib are inhibitors of the cyclin-dependent kinases 4 and 6 which have been approved for the treatment of locally advanced or metastatic breast cancer. In this review, we provide an overview of the available clinical pharmacokinetic and pharmacodynamic characteristics of these novel drugs, summarize the results of food-effect and drug-drug interaction studies and highlight exposure-response and exposure-toxicity relationships. All three drugs exhibit a large interindividual variability in exposure (coefficient of variation ranging from $40-95 \%$ for minimum plasma concentration $\left.\left(C_{\mathrm{min}}\right)\right)$, are extensively metabolized by cytochrome P450 3A4 and have their brain penetration limited by efflux transporters. Abemaciclib has three active metabolites with similar potency that are clinically relevant (i.e. M2, M20, M18), whereas the metabolites of palbociclib and ribociclib are not of clinical significance. Pharmacokinetic exposure increases in a dose-proportional manner for palbociclib, whereas exposure increases under- and over-proportionally with increasing dose for abemaciclib and ribociclib, respectively. High exposure is associated with an increased risk of neutropenia, and for ribociclib also to QTc prolongation. For abemaciclib, a clear exposure-efficacy relationship has been described, while for palbociclib and ribociclib exposure-response analyses remain inconclusive. Future studies are needed to address exposure-efficacy relationships to further improve dosing. 


\section{INTRODUCTION}

Cyclin-dependent kinase 4 and 6 (CDK4/6) inhibitors have emerged as important targeted therapies in the treatment of patients with advanced breast cancer. CDK4/6 inhibitors act on the cell cycle and prevent G1-to-S-phase progression. In order for cells to proceed past this G1-to-S-phase checkpoint, retinoblastoma protein $(\mathrm{Rb})$ needs to be phosphorylated, which is effectuated by CDK4/6. ${ }^{1}$ Aberrations in this pathway are often involved in carcinogenesis, resulting in persistent cell proliferation. ${ }^{2}$ Treatment with CDK4/6 inhibitors prevents phosphorylation of Rb and thereby causes a G1 cell cycle arrest, blocking cell division (Figure 1).

As of today, three CDK4/6 inhibitors are available in the clinic (i.e. palbociclib, ribociclib and abemaciclib), and many more are in (pre)clinical development (Table 1). Although all three CDK4/6 inhibitors are approved for treatment in combination with endocrine therapies (i.e. aromatase inhibitors or fulvestrant), only abemaciclib is registered to use as monotherapy. In general, the efficacy of CDK4/6 inhibitors is strikingly consistent between endocrine partners and clinical settings with respect to improved progressionfree survival (PFS), and emerging evidence of overall survival (OS) benefit, but their toxicity differs. The aim of this review is to summarize the available clinical pharmacokinetic and pharmacodynamic data on the currently approved CDK4/6 inhibitors palbociclib, ribociclib and abemaciclib. In addition, we will focus on exposure-response relationships and the potential for pharmacokinetically guided dose individualization.

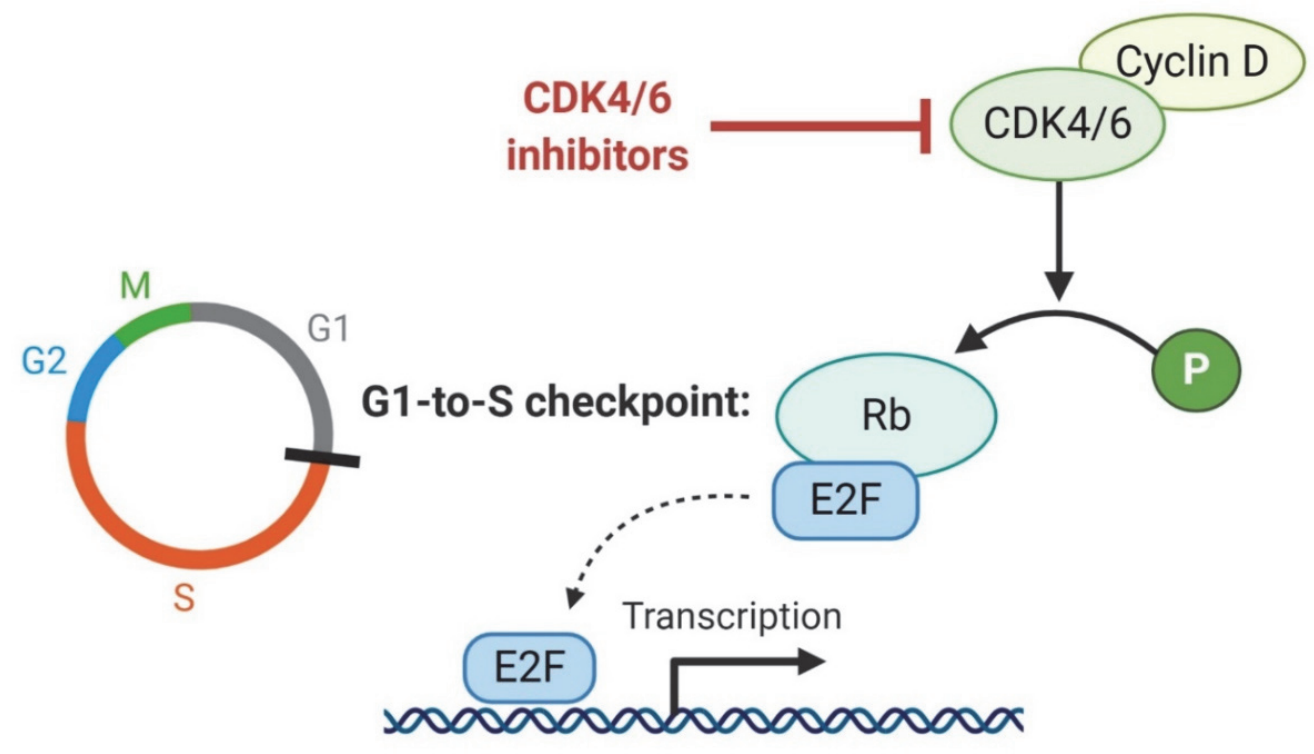

\section{Figure 1 - Mechanism of action of CDK4/6 inhibitors}

For cells to progress from $\mathrm{G} 1$ to $\mathrm{S}$ phase in the cell cycle, Rb needs to get phosphorylated, which is catalyzed by the complex formed by CDK4/6 and cyclin D. Upon phosphorylation of Rb, the transcription factor E2F is released, ultimately resulting in cells proceeding to S phase. CDK4/6 inhibitors prevent $\mathrm{Rb}$ from getting phosphorylated and thereby block cell cycle progression. Created with BioRender.com.

$C D K=$ cyclin-dependent kinases; $P=$ phosphoryl $\left.\left(\mathrm{PO}_{3}\right)^{-}\right) ; \mathrm{R} b=$ retinoblastoma 


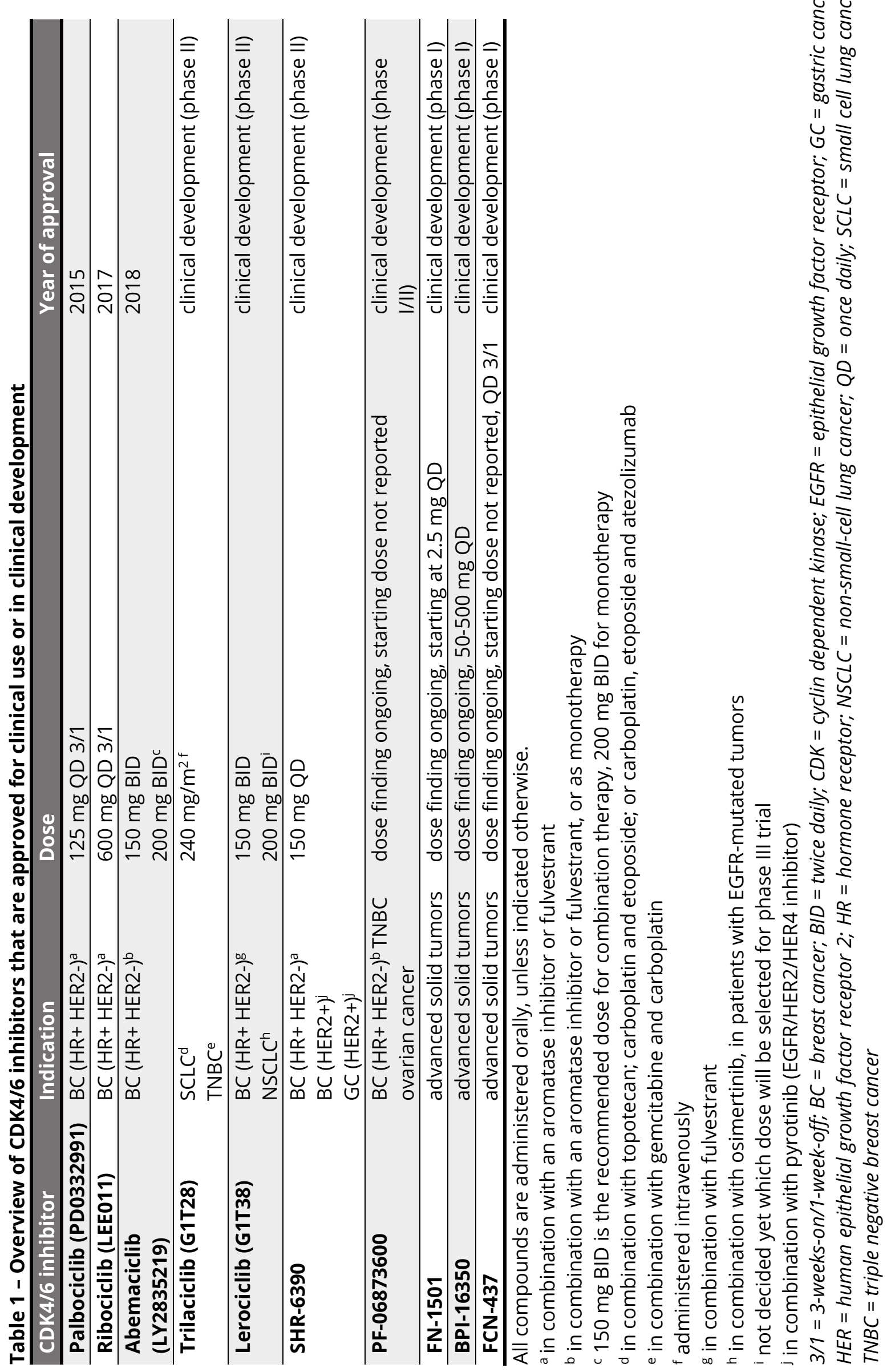




\section{PALBOCICLIB}

Palbociclib was the first CDK4/6 inhibitor to obtain approval by the Food and Drug Administration (FDA) and European Medicines Agency (EMA) in 2015. In the pivotal PALOMA-2 study, the addition of palbociclib to letrozole as first-line treatment for hormone receptor (HR) positive, human epidermal growth factor receptor 2 (HER2)negative advanced breast cancer patients resulted in a median progression-free survival (mPFS) of 24.8 months compared with 14.5 months for letrozole alone (HR 0.58 (95\% confidence interval $(\mathrm{Cl}): 0.46-0.72), \mathrm{p}<0.001){ }^{3}$ Similarly, the PALOMA-3 study demonstrated that palbociclib and fulvestrant were superior to fulvestrant alone in patients who progressed on $\geq 1$ prior lines of treatment (mPFS 9.2 vs. 3.8 months, HR 0.42 (95\% Cl: 0.32-0.56), p < 0.001). ${ }^{4}$

The approved dose of palbociclib is $125 \mathrm{mg}$ once daily (QD) in a 3-weeks-on/1-week-off dosing schedule. This was also the maximum tolerated dose (MTD), with neutropenia being the only dose-limiting toxicity (DLT). ${ }^{5}$

\section{Physiochemical properties and formulation}

Palbociclib is a synthetic 6-acetyl-8-cyclopentyl-5-methyl-2-(5-piperazin-1-yl-pyridin-2ylamino)-8H-pyrido[2,3-d]pyrimidin-7-one, which belongs to the class of pyridopyrimidines (Figure 2). ${ }^{7}$ Palbociclib is a weak base with two pKa values of 3.9 and 7.4, and a calculated log octanol-water partition coefficient (cLogP, which is an indicator of lipophilicity) of 2.7.8,9 Palbociclib is highly soluble at $\mathrm{pH}<4$, but its solubility rapidly decreases at higher $\mathrm{pH} .{ }^{9}$ For drugs to be classified as high solubility compounds, their highest approved dose needs to be soluble in $\leq 250 \mathrm{~mL}$ of aqueous media (i.e. $\geq 0.5 \mathrm{mg}$ / $\mathrm{mL}$ for palbociclib) over the entire $\mathrm{pH}$ range of 1.0-6.8. ${ }^{10}$ Therefore, palbociclib is considered a low solubility compound. Together with its high permeability, palbociclib is classified as a class II compound, according to the Biopharmaceutics Classification System (BCS). ${ }^{9}$ Initially, palbociclib free base was formulated in capsules, but recently a bioequivalent tablet formulation was approved, containing the free base as well. ${ }^{11}$

In vitro, palbociclib bound reversibly to its targets and the half-maximal inhibitory concentrations ( $\mathrm{IC}_{50}$ ) were 0.011 and $0.016 \mu \mathrm{M}$ for CDK4 and CDK6, respectively, corresponding to plasma concentrations of $33.5-48.7 \mathrm{ng} / \mathrm{mL}$ when corrected for protein binding. ${ }^{12}$

\section{Drug transporters}

In vitro assays demonstrated that palbociclib is a substrate of the efflux transporters P-glycoprotein (P-gp) and breast cancer resistance protein 
(BCRP). ${ }^{13,14}$ Although this only marginally affected the oral bioavailability in in vivo experiments with P-gp and/or BCRP knock-out mice, it has been demonstrated that the brain penetration was drastically restricted by these transporters. ${ }^{13}$

In addition, in vitro and in vivo studies have shown that palbociclib inhibits the organic cation transporter $2(\mathrm{OCT} 2)^{15}$, which is involved in the renal tubular secretion of creatinine. Although this has not been studied in patients treated with palbociclib, inhibition of the OCT2 transporter has been associated with an increase in creatinine levels without affecting glomerular filtration. ${ }^{16}$

\section{Clinical pharmacokinetics}

Table 2 provides and overview of selected steady-state pharmacokinetic parameters of palbociclib. The bioavailability of palbociclib is low (46\%). ${ }^{9}$ Palbociclib has a large volume of distribution of $\sim 2800 \mathrm{~L}$ and the total plasma protein binding is $85.3 \%$, with similar binding to albumin and a1-acid glycoprotein. 5,9 Metabolism mainly takes place by cytochrome P450 3A4 (CYP3A4) and sulfotransferase 2A1 (SULT2A1) and results in the formation of many metabolites, of which M22 (i.e. palbociclib glucuronide) is the most abundant (14.8\%) and M17 (i.e. a lactam of palbociclib) is pharmacologically active with a similar potency as palbociclib, but accounting for less than $10 \%$ of total plasma exposure (Figure 2). ${ }^{9}$ Hepatic metabolism is the main route of elimination, as in the mass-balance study $74.1 \%$ of palbociclib was excreted in feces compared with $17.5 \%$ in urine, including both unchanged palbociclib and metabolites. ${ }^{9}$

\section{Pharmacokinetics in special populations}

\section{Pediatric cancer patients}

As of to date, palbociclib is not approved for the treatment of pediatric cancer and hence no pharmacokinetic data is available in this subgroup. ${ }^{17}$ Several phase I-II studies in pediatric patients are currently ongoing. ${ }^{18-22}$

\section{Patients with renal impairment}

In a clinical study, subjects with mild (estimated glomerular filtration rate (eGFR) 60-90 $\mathrm{mL} / \mathrm{min} / 1.73 \mathrm{~m}^{2}$ ), moderate (eGFR 30-60 mL/min/1.73 ${ }^{2}$ ) and severe (eGFR $<30 \mathrm{~mL} /$ $\mathrm{min} / 1.73 \mathrm{~m}^{2}$ ) renal impairment showed an increase in palbociclib $\mathrm{AUC}_{0-\infty}$ of $39 \%, 42 \%$ and $31 \%$, respectively, compared with patients with a normal renal function. Similarly, $C_{\max }$ was $17 \%, 12 \%$ and $15 \%$ higher, respectively. ${ }^{23}$ In a population pharmacokinetic analysis ( $n=183$, of whom $n=73$ and $n=29$ with mild and moderate renal impairment, respectively), creatinine clearance did not significantly affect palbociclib exposure, which is consistent with renal clearance being a minor route of elimination. ${ }^{9}$ No data are available for patients 
requiring hemodialysis, but based on the large fraction of palbociclib bound to plasma proteins (i.e. 85.3\%), hemodialysis is expected to have limited effect on palbociclib exposure. ${ }^{9}$ In conclusion, no dose adjustments are needed for patients with an eGFR $\geq 15$ $\mathrm{mL} / \mathrm{min} / 1.73 \mathrm{~m}^{223}$, but it should be kept in mind that exposure is $30-40 \%$ higher in patients with renal impairment.

\section{Patients with hepatic impairment}

Palbociclib unbound $\mathrm{AUC}_{0-\infty}$ was $17 \%$ lower in subjects with mild hepatic impairment (Child-Pugh class A) and 34\% and 77\% higher in patients with moderate (Child-Pugh class B) and severe (Child-Pugh class C) hepatic impairment, respectively, compared with subjects with a normal hepatic function. Unbound $C_{\max }$ was increased by $7 \%, 38 \%$ and $72 \%$, respectively. ${ }^{23}$ These findings are in line with the fact that hepatic clearance is the major route of elimination, and

were also supported by population pharmacokinetic analyses. ${ }^{9}$ Based on the above, no dose adjustments are needed for patients with mild or moderate hepatic impairment, while a dose reduction from $125 \mathrm{mg}$ (standard dose) to $75 \mathrm{mg}$ QD is recommended for patients with severe hepatic impairment. ${ }^{23}$ It has to be noted that interpretation of palbociclib plasma concentrations in this subgroup could be complicated by the increasing fraction unbound with worsening hepatic function, because this might not be reflected in the total concentration, which is usually measured. ${ }^{23}$ In addition, caution is warranted when using the Child Pugh score in patients with cancer, as this score has not been developed nor validated for this population. ${ }^{24}$

\section{Other factors influencing palbociclib pharmacokinetics}

The effect of other intrinsic factors on palbociclib exposure was investigated using a population pharmacokinetic model. Age and body weight were significant covariates on palbociclib clearance, which was higher in younger patients and in patients with a higher body weight (i.e. compared with a typical patient of 61 years and $73.7 \mathrm{~kg}$, clearance was increased by $14.7 \%$ and decreased by $8.33 \%$ in a 45 -year-old and a 97 -year-old subject, respectively, while for body weight clearance was decreased by $13.2 \%$ at a weight of $55 \mathrm{~kg}$ and increased by $14.2 \%$ at a weight of $97 \mathrm{~kg}$ ), although these small differences are not expected to be clinically relevant. Gender had no effect on palbociclib exposure. ${ }^{9}$

In a subgroup analysis of the PALOMA-2 study, palbociclib exposure was higher in Japanese and other Asian patients compared to non-Asian patients (geometric mean $\mathrm{C}_{\text {min }}$ 95.4 ng/mL and $90.1 \mathrm{ng} / \mathrm{mL}$ vs. $61.7 \mathrm{ng} / \mathrm{mL}$ ), whereas in a similar analysis of the PALOMA3 study no difference was found. ${ }^{25,26}$ In another study ( $\left.n=25\right), A \cup C_{0-\infty}$ and $C_{\max }$ were $30 \%$ and $35 \%$ higher, respectively, in Japanese subjects. ${ }^{27}$ No dose adjustments are recommended based on ethnicity. ${ }^{9}$ 


\section{Food effect}

Food-effect studies of capsule and tablet formulations of palbociclib are summarized in Table 3. In a previous pooled analysis it has been demonstrated that palbociclib exposure is substantially lower in a subset of patients (i.e. 13\%), possibly due to a decreased absorption caused by an elevated stomach $\mathrm{pH}$. This subgroup is classified as low-liers, defined as $C_{\max }<21.4 \mathrm{ng} / \mathrm{mL}^{9}{ }^{9}$ In the food-effect study, when the patients who met the low-lier criteria were excluded, $90 \% \mathrm{Cls}$ were within the bioequivalence margins, implying no food-effect in patients with adequate absorption. ${ }^{28}$

Concomitant intake with food resulted in a reduced interindividual variability, because the small subset of low-liers now leveled up to the exposure of the rest of the population, supporting the recommended ingestion of palbociclib capsules together with a meal. ${ }^{28}$

While palbociclib capsules need to be administered with food, the recently approved tablet formulation can be taken with or without food, offering more flexibility to patients. Palbociclib exposure was not significantly altered due to food intake using the tablet formulation, showing to be more robust to $\mathrm{pH}$ differences. ${ }^{11}$

\section{Drug-drug interactions}

In Table 4, results of drug-drug interaction studies and recommendations for dose adjustments are shown. Overall, no (clinically relevant) interactions with fulvestrant, goserelin or aromatase inhibitors were found. In contrast, palbociclib exposure was significantly altered by strong CYP3A4 modulators.

No clinical studies have been executed for moderate CYP3A4 inhibitors, but simulations predicted that they would increase palbociclib $C_{\max }$ and $A \cup C_{0-\infty}$ by approximately $23 \%$ and $40 \%$, respectively. ${ }^{29}$ According to the label, no dose reduction is warranted although these results suggest that a dose reduction from $125 \mathrm{mg}$ (standard dose) to $100 \mathrm{mg}$ QD might be advised. To further substantiate this finding, we are currently performing a clinical study to investigate the effect of the moderate CYP3A4 inhibitor erythromycin on the pharmacokinetics of Palbociclib. ${ }^{30}$ In addition, relevant interactions between palbociclib and CYP3A4 substrates with a narrow therapeutic index could occur, since palbociclib can weakly inhibit CYP3A4. ${ }^{31}$

As the solubility of palbociclib is $\mathrm{pH}$-dependent, it could be expected that acid-reducing agents would decrease its exposure. Although palbociclib exposure was substantially reduced when administered concomitantly with rabeprazole under fasted conditions, this effect was only modest under fed conditions (Table 4). ${ }^{32}$ Therefore, no dose adjustments are indicated when palbociclib capsules are co-administered with acid-reducing agents, as they have to be administered under fed conditions. Exposure of palbociclib tablets was not affected by acid-reducing agents. ${ }^{11}$ 


\section{Pharmacokinetic-pharmacodynamic relationships}

\section{Exposure-response}

Initial exposure-response analyses based on data of the PALOMA-1 study were inconclusive due to limited data $(n=81)$. Although a trend for prolonged PFS was observed in patients with an average palbociclib concentration ( $C_{\text {avg }}$ ) above the median of $60 \mathrm{ng} / \mathrm{mL}$ (median PFS estimated from Kaplan Meier curves were 17 months vs. 24.5 months, pvalue not reported), multivariable analyses yielded inconsistent results. ${ }^{9}$

In the PALOMA-3 study, PFS was similar in patients with $\mathrm{C}_{\text {avg }}$ above and below the median of $78 \mathrm{ng} / \mathrm{mL}$. It has to be noted, though, that exposure in this trial appeared to be higher than in PALOMA-1 (at the same dose, but with fulvestrant instead of aromatase inhibitors). Even in the group with low exposure, median $C_{\text {avg }}$ was $63 \mathrm{ng} / \mathrm{mL}$, which is higher than the cut-off value used in the PALOMA-1 study. Time-varying $C_{\text {avg }}$ as a continuous variable was a significant predictor of PFS in univariable analysis, although this did not remain significant in multivariable analysis. ${ }^{27,33}$

In the PALOMA-2 study, no exposure-response relationship has been identified..$^{34,35}$

As exposure-response analyses have thus far not resulted in a clear answer and optimal data to perform them were not available, this needs to be further elucidated. Lower thresholds of $C_{\min }$ may be related to efficacy. Preferably, these additional analyses should include palbociclib plasma concentrations measured at regular intervals throughout treatment and use median $\mathrm{C}_{\min }$ as a measure of exposure. Previously, it has been suggested that individual concentrations could be compared to the mean $C_{\min }$ of $61 \mathrm{ng} / \mathrm{mL}$ of the PALOMA-1 study. ${ }^{36}$

\section{Exposure-toxicity}

In phase I studies higher AUC values were related to a greater reduction in absolute neutrophil count and platelet levels, with a wide range in $\mathrm{EC}_{50}$ values (estimated plasma exposure resulting in 50\% decrease from baseline) varying from $253-716 \mathrm{ng} / \mathrm{mL}$ *h for neutropenia and 184-1370 ng/mL*h for thrombocytopenia. ${ }^{5,6}$

A semi-mechanistic pharmacokinetic-pharmacodynamic model has been developed to quantify the relationship between palbociclib exposure (i.e. plasma concentration) and neutropenia. ${ }^{37}$ In this model, the maximum anti-proliferative effect on neutrophil precursor cells $\left(E_{\max }\right)$ was notably lower than for cytotoxic drugs (e.g. docetaxel and etoposide), and the reported $\mathrm{EC}_{50}$ value was $40.1 \mathrm{ng} / \mathrm{mL}$.

Interestingly, patients with grade 3 or 4 neutropenia had a significantly longer PFS compared with patients with lower grade or no neutropenia $(p=0.0046)$. Multivariable analysis resulted in a hazard ratio of $0.502(95 \% \mathrm{Cl} 0.26-0.98, p=0.046)$. This could be explained by either the hypothesis that tumor cells in patients with neutropenia are more 
sensitive to palbociclib or an underlying higher exposure in these patients. ${ }^{9}$ As higher drug exposure causes more neutropenia and more neutropenia is related to a prolonged PFS, this suggests that an exposure-response relationship exists.

\section{Population pharmacokinetic models}

In a population pharmacokinetic model palbociclib pharmacokinetics was best described by a two-compartment model with first-order absorption. ${ }^{9}$ Two additional models have been developed to predict drug-drug interactions with CYP3A4 inhibitors and to quantify the exposure-response relationship for neutropenia. ${ }^{29,37}$

\section{RIBOCICLIB}

In 2017, ribociclib has been approved by the FDA and EMA based on the results of a preplanned interim analysis of the MONALEESA- 2 study. ${ }^{38}$ In the second interim analysis of this randomized placebo-controlled phase III trial $(n=668)$ comparing first-line treatment with letrozole with or without ribociclib, mPFS was significantly longer in the ribociclib group compared with the control group (25.3 vs. 16 months, HR 0.57 (95\% Cl: 0.46-0.70), $p<0.001) .{ }^{39}$ In 2018, the indication was extended to combination treatment with fulvestrant, based on the MONALEESA-3 study. This study revealed that addition of ribociclib to the treatment of fulvestrant, improved mPFS from 12.8 to 20.5 months (HR 0.60 (95\% Cl: 0.48-0.73), $\mathrm{p}<0.001)$ and resulted in a prolonged median OS (40.0 months vs. not reached yet (HR 0.72 (95\% Cl: 0.57-0.92), $\mathrm{p}=0.005) .{ }^{40,41}$

The approved dose of ribociclib is $600 \mathrm{mg}$ QD in a 3-weeks-on/1-week-off dosing schedule. In the phase I dose escalation study, doses ranging from 50-1200 mg QD were explored. ${ }^{42}$ The MTD was established as $900 \mathrm{mg}$ QD, with neutropenia and thrombocytopenia being the most common DLTs. ${ }^{42}$ The lower dose level of $600 \mathrm{mg}$ QD was selected for further development, as this resulted in a lower rate of QTc prolongation, and clinical activity was already observed at this dose level. ${ }^{42}$

\section{Physiochemical properties and formulation}

Ribociclib is a 7-cyclopentyl- $N, N$-dimethyl-2-\{[5-(piperazin-1-yl)pyridine-2-yl]amino\}-7Hpyrrolo[2,3-d]pyrimidine produced by chemical synthesis. It is formulated as a succinate salt in film-coated tablets containing $200 \mathrm{mg}$ of ribociclib free base. Although initially a capsule formulation was used in clinical trials, the equivalence of both dosage forms was demonstrated in a bioequivalence study. ${ }^{43}$ Ribociclib is a weak base with two $\mathrm{p} K_{\mathrm{a}}$ values of 5.5 and 8.6, with its succinate salt exhibiting high solubility at $\mathrm{pH} \leq 4.5$ (solubility $>2.4$ $\mathrm{mg} / \mathrm{mL}$ ), but it decreases at higher $\mathrm{pH}$. Thus, ribociclib succinate was classified as a low solubility compound. ${ }^{43,44}$ The ribociclib Log P was reported to be 1.95 , and its estimated effective human permeability $\left(\mathrm{hP}_{\text {eff }}\right.$ ) was $0.9 * 10^{-4} \mathrm{~cm} / \mathrm{s}$. Based on these data, it was categorized in BCS class IV (low solubility, low permeability). ${ }^{43,45}$ 
In vitro, $\mathrm{IC}_{50}$ values for ribociclib were 8 and $39 \mathrm{nM}$ for CDK4 and CDK6, respectively, corresponding to plasma concentrations of $11.6-56.5 \mathrm{ng} / \mathrm{mL}$ when corrected for protein binding. ${ }^{46}$

\section{Drug transporters}

In vitro and in vivo studies have demonstrated that ribociclib is a transport substrate of $\mathrm{P}$ gp. ${ }^{47,48}$ Interestingly, pharmacokinetic and tissue distribution studies in mouse models showed that this efflux transporter is responsible for limiting the ribociclib penetration into the brain, since the brain-to-plasma concentration ratio increased by at least 23-fold when the P-gp was knocked out or inhibited. Plasma pharmacokinetic parameters were not significantly affected, except for $\mathrm{AUC}_{0-24 \mathrm{~h}}$, which increased 2.3-fold in mice lacking the $\mathrm{P}$-gp and BCRP. This increase is likely due to P-gp activity, since ribociclib has not shown noticeable transport by BCRP. ${ }^{47}$

Besides interacting as a substrate, ribociclib also inhibited P-gp. ${ }^{48}$ Moreover, at clinically relevant concentrations it also inhibited $B C R P, O C T 2$, multidrug and toxin extrusion protein (MATE) 1, and bile salt export pump (BSEP). ${ }^{15,46,48,49}$ In a retrospective study in patients treated with ribociclib, creatinine levels increased $37 \%$ compared to baseline, probably due to OCT2 inhibition. ${ }^{50}$

\section{Clinical pharmacokinetics}

Selected steady-state pharmacokinetic parameters of ribociclib are displayed in Table 2. Exposure of ribociclib increased over-proportionally with dose in the range of 50-1200 mg, possibly caused by a lower clearance at higher doses. ${ }^{51}$

The absolute oral bioavailability has not been determined, but using a physiologicallybased pharmacokinetic (PBPK) model it was predicted that $90 \%$ of the standard dose of ribociclib (600 $\mathrm{mg}$ ) would be absorbed mainly in the small intestine. ${ }^{44}$ Ribociclib has a moderate human protein binding ( $\pm 70 \%)$, and is equally distributed in plasma and red blood cells. The apparent volume of distribution was estimated at $1090 \mathrm{~L}$, using a population pharmacokinetic analysis. ${ }^{46,49}$

Ribociclib is metabolized primarily by CYP3A4 with the formation of the active metabolite M4 (Figure 2). It is also metabolized to a minor extent by flavin-containing monooxygenase (FMO) 3 and FMO1, the last being involved in the formation of the metabolite M13. These two metabolites may be reactive by forming covalent adducts in hepatocytes. M4, M13 and M1 (a secondary glucuronide of ribociclib) were the major circulating metabolites, accounting for, respectively, $8.6 \%, 9.4 \%$ and $7.8 \%$ of total radioactivity in a mass balance study. Considering these data, the contribution of the active metabolite M4 to the clinical activity was considered negligible. ${ }^{46,49,52}$ 
Feces was the major route of excretion compared to urine, accounting for, respectively, $69.1 \%$ and $22.6 \%$ of the dose recovered, where ribociclib was the major entity found in excreta. ${ }^{46,49}$

\section{Pharmacokinetics in special populations}

\section{Pediatric cancer patients}

Ribociclib was the first CDK4/6 inhibitor studied in pediatric patients in a phase I clinical trial, where patients with neuroblastoma, rhabdoid tumors or solid tumors with alterations in the cyclin D-CDK4/6-INK4-Rb pathway were included. The MTD and recommended phase II dose (RP2D) were 470 and $350 \mathrm{mg} / \mathrm{m}^{2}$, respectively. The RP2D dose was selected based on overall safety and pharmacokinetic considerations, since the exposure at $350 \mathrm{mg} / \mathrm{m}^{2}$ was equivalent to that observed at $600 \mathrm{mg}$ in adults. Pharmacokinetic parameters, including $t_{\max }, C_{\max }, A \cup C_{0-\tau}$ and $t_{1 / 2}$, were similar in adults and pediatric patients. ${ }^{53}$

\section{Patients with renal impairment}

The effect of renal impairment on the pharmacokinetics of ribociclib was assessed in a population pharmacokinetic analysis, which included patients with normal renal function $(n=438)$, mild renal impairment $(n=488)$ and moderate renal impairment $(n=113)$. In this analysis, mild and moderate renal impairment had no effect on the exposure and clearance of ribociclib, therefore no dose adjustments are recommended for patients with mild or moderate renal impairment. ${ }^{46,49,52}$

Furthermore, a clinical trial showed that for patients with severe renal impairment and end stage renal disease (eGFR $<15 \mathrm{~mL} / \mathrm{min} / 1.73 \mathrm{~m}^{2}$ ), $\mathrm{AUC}_{0-\infty}$ increased $281 \%$ and $137 \%$, and $C_{\max } 168 \%$ and $110 \%$, respectively, compared to subjects with normal renal function. ${ }^{54}$ Based on these results, a starting dose of $200 \mathrm{mg}$ daily is recommended by the FDA for patients with severe renal impairment, while the EMA recommends a starting dose of 400 $\mathrm{mg}$ in these cases. ${ }^{52,55}$

\section{Patients with hepatic impairment}

In a clinical study ( $n=28)$, mild hepatic impairment had no effect on ribociclib exposure. In contrast, for moderate hepatic impairment, $A \cup C_{0-\infty}$ and $C_{\max }$ increased $28 \%$ and $44 \%$, respectively, while for severe hepatic impairment they increased 29 and 32\%. A population pharmacokinetic analysis ( $n=160$ with normal hepatic function and $n=47$ with mild hepatic impairment) further supported that ribociclib exposure was unaffected by mild hepatic impairment. Based on these results, a reduction of the starting dose to 400 $\mathrm{mg}$ is recommended for patients with moderate or severe hepatic impairment. ${ }^{46,49}$ 


\section{Other factors influencing ribociclib pharmacokinetics}

The effect of other intrinsic factors on ribociclib pharmacokinetics was evaluated by population pharmacokinetic analyses $(n=208)$. Body weight and age were statistically significant covariates for ribociclib clearance. Based on simulations, it was predicted that a change of body weight from the reference value of $70 \mathrm{~kg}$ to 50 or $100 \mathrm{~kg}$ would change steady state $C_{\max }, C_{\min }$, and $A \cup C_{0-24 h}$ of ribociclib up to $22 \%$, which was considered a small effect relative to the inherent pharmacokinetic variability. Age was predicted to have only a mild effect on exposure. Race and gender were statistically insignificant parameters. ${ }^{46}$

Furthermore, a cross-study comparison exhibited that, on average, the exposure of ribociclib in Japanese patients was higher than in Caucasian patients, but the individual values were within the same range. In summary, the effects of body weight, age, gender and race on ribociclib pharmacokinetics were considered not clinically relevant, and therefore, no dose adjustment is required. ${ }^{46,49}$

\section{Food effect}

Table 3 summarizes the food-effect studies that have been performed for the capsule and tablet formulation, of which the latter is more relevant since this is the marketed formulation. Since the geometric mean ratios were $\approx 1$ for $A \cup C_{0-\infty}$ and $C_{\max }$, no effect of food intake was observed on the pharmacokinetics of ribociclib. ${ }^{44}$

Additionally, the in vitro solubility of ribociclib was evaluated in biorelevant media, including simulated fed ( $\mathrm{pH}$ 5.0) and fasted ( $\mathrm{pH}$ 6.5) intestinal fluid, where the maximum dose (600 mg) was dissolved in $250 \mathrm{~mL}$. This suggests that ribociclib absorption is unlikely to be affected by changes in the gastric $\mathrm{pH}$ due to food intake, among others. PBPK models also predicted that the exposure of ribociclib was independent of the gastric $\mathrm{pH}$ in the range of 1.0-8.0.44 Altogether, this information supports that ribociclib can be administered either with or without food. ${ }^{46,49,52}$

\section{Drug-drug interactions}

An overview of all drug-drug interaction studies is provided in Table 4. Ribociclib had no (clinically relevant) interactions with fulvestrant and the aromatase inhibitors. ${ }^{46,49,52,55}$ Ribociclib is extensively metabolized via CYP3A4, therefore its pharmacokinetics is strongly affected by strong inhibitors or inducers of this enzyme. Ribociclib can reversibly inhibit CYP3A4 and CYP1A2. ${ }^{48}$ Altogether, it is recommended that drugs with narrow therapeutic index that are sensitive substrates of these drug-metabolizing enzymes or transporters which are inhibited by ribociclib (section 3.2) should be cautiously monitored in concomitant treatments with ribociclib. ${ }^{46,49}$

Since ribociclib shows a $\mathrm{pH}$-dependent solubility, drugs that alter the gastric $\mathrm{pH}$ could be expected to affect its exposure. However, ribociclib exposure was similar in patients with 
and without a proton pump inhibitor, and these drugs could thus be administered concomitantly. ${ }^{44}$

\section{Pharmacokinetic-pharmacodynamic relationships}

\section{Exposure-response}

Due to very limited data, exposure-response analyses for ribociclib remain inconclusive. In the MONALEESA-2 study only 44 out of 334 patients had progressive disease and available pharmacokinetic data. No indication for an exposure-efficacy relationship was found. Data on confirmed best response were available for 72 patients with pharmacokinetic data, and showed similar ribociclib exposure in responders vs. nonresponders. No exposure-response analyses have been performed for the MONALEESA3 study. ${ }^{46,56}$ Future studies should establish exposure-efficacy relationships and identify an optimal threshold concentration.

\section{Exposure-toxicity}

Although higher $C_{\min }$ levels were related to a greater decrease in absolute neutrophil count and platelet count in the phase I study, ribociclib is dosed at the flat ends of these plateauing curves. ${ }^{42}$ Pooled data from four clinical studies $(n=196)$ were used to develop a logistic regression model for $\geq$ grade 2 neutropenia. Although a trend was found for an increased risk of neutropenia at higher ribociclib exposure, this was not statistically significant. For each $100 \mathrm{ng} / \mathrm{mL}$ increase in $\mathrm{C}_{\min }$ the odds ratio for $\geq$ grade 2 neutropenia was 1.05 (95\% Cl: 0.99-1.11, p=0.087). ${ }^{46}$ In addition, a pharmacokinetic-pharmacodynamic model for neutropenia using data of 1052 patients from six clinical trials showed that the relationship between exposure and neutropenia was not influenced by age, race, or the use of anastrozole, letrozole, tamoxifen or fulvestrant. ${ }^{56}$

Furthermore, a relationship between ribociclib exposure and QTc prolongation has been established, which was described by a log-linear mixed effect model. Mean QTC prolongation was $22.87 \mathrm{~ms}$ at the mean steady-state $C_{\max }$ of $2237 \mathrm{ng} / \mathrm{mL}$. No exposuretoxicity relationship could be demonstrated for hepatotoxicity, due to the limited number of grade 3 or 4 events. ${ }^{46}$

\section{Population pharmacokinetic models}

A population pharmacokinetic model has been developed based on pooled data of 208 patients of whom 4731 pharmacokinetic samples were available. The model was validated using a dataset consisting of 175 pharmacokinetic samples from 93 patients in the MONALEESA-2 study. A two-compartment model with delayed zero-order absorption and linear clearance best fitted the data, with dose and body weight being significant covariates on clearance. Clearance decreased with increasing dose, which is in line with the observed more than dose-proportional increase in exposure. ${ }^{46}$ 


\section{ABEMACICLIB}

Abemaciclib was the third CDK4/6 inhibitor approved by the FDA and EMA in 2018. In the MONARCH-3 study, abemaciclib increased mPFS compared to placebo (14.7 months vs. not reached, HR 0.54 (95\% Cl: 0.41-0.72), p < 0.001) in the first-line setting combined with anastrozole or letrozole. ${ }^{57}$ In the same way, the MONARCH-2 study demonstrated that abemaciclib was superior to placebo in the second-line setting in combination with fulvestrant. ${ }^{58}$

In contrast to palbociclib and ribociclib, abemaciclib is dosed twice daily (BID) and in a continuous dosing schedule. In the dose escalation part of the phase I study, doses up to $275 \mathrm{mg}$ BID have been evaluated with $200 \mathrm{mg}$ BID being identified as MTD. ${ }^{59}$ This is the recommended dose for abemaciclib monotherapy, whereas $150 \mathrm{mg}$ BID is the recommended dose for combination therapy (i.e. with aromatase inhibitors or fulvestrant), because of better tolerability. Although fatigue was the most common DLT, gastrointestinal and hematological toxicities were also frequently observed. ${ }^{59}$

\section{Physiochemical properties and formulation}

Abemaciclib is a synthetic N-(5-((4-ethylpiperazin-1-yl)methyl)pyrididin-2-yl)-5-fluoro-4-(4fluoro-1-isopropyl-2-methyl-1H-benzo[d]imidazole-6-yl)pyrimidin-2-amine. It is formulated in tablets containing 50,100, 150 or $200 \mathrm{mg}$ of the free base. Since capsules were used in the pivotal MONARCH-1 and MONARCH-2 trials, bioequivalence between both formulations was tested and confirmed. Abemaciclib is a tribasic compound with $\mathrm{p} K_{a}$ values of 3.80, 4.48 and 7.95, and a log $\mathrm{P}$ of 3.36. It is soluble over the $\mathrm{pH}$ range of 1.0-6.8 (solubility $>0.8 \mathrm{mg} / \mathrm{mL}$ ), and classified as a highly soluble drug. Considering that abemaciclib showed a moderate permeability (predicted $h P_{\text {eff }}=2.46 * 10^{-4} \mathrm{~cm} / \mathrm{s}$ ), it was classified as a BCS class 3 (high solubility, low permeability) drug. ${ }^{60,61}$

Abemaciclib is a potent, ATP-competitive, reversible inhibitor of CDK4 and CDK6, with IC 50 values of 2 and $10 \mathrm{nM}$, respectively, corresponding to plasma concentrations of 27.4-136.9 $\mathrm{ng} / \mathrm{mL}$ after correcting for protein binding. ${ }^{62}$ Abemaciclib has three active metabolites with similar potency: N-desethylabemaciclib (M2), hydroxyabemaciclib (M20) and hydroxy-N-desethylabemaciclib (M18) (Figure 2). Their $I_{50}$ values (nM) for CDK4 and CDK6 are 1.2 and 1.3 for M2,1.5 and 1.9 for M20 and 1.5 and 2.7 for M18. ${ }^{63,64}$

\section{Drug transporters}

Abemaciclib is a substrate of efflux transporters P-gp and BCRP. In vivo studies showed that abemaciclib penetration through the blood-brain barrier improved in P-gp deficient mice. ${ }^{14}$ The abemaciclib metabolite $M 2$ is also a substrate of P-gp and BCRP, and its exposure increased significantly around 5.3-fold in P-gP/BCRP deficient mice with respect to the wild-type. Also, in this mouse model the brain penetration of both abemaciclib and 
M2 increased 25- and 4-fold, respectively, compared to the wild-type. ${ }^{65}$ Additionally, abemaciclib itself inhibits P-gp and BCRP. ${ }^{66,67}$

The renal transporters OCT2, MATE1 and MATE2-K are reversibly inhibited by abemaciclib and its active metabolites $\mathrm{M} 2$ and $\mathrm{M} 20$ at clinically relevant concentrations. ${ }^{67,68}$ In vitro studies have shown that OCT2, MATE1 and MATE-K metformin transport is inhibited in the presence of abemaciclib, M2 or M20. The clinical implications of this interaction were also determined (Table 4). ${ }^{68}$ This reversible inhibition of renal transporters has been related to elevated creatinine levels, without renal function being affected. ${ }^{68}$

\section{Clinical pharmacokinetics}

Abemaciclib pharmacokinetics is summarized in Table $\mathbf{2}$, and is characterized by a high variability. It showed a relatively modest absolute oral bioavailability of $45 \% .59,69,70$ Abemaciclib and its active metabolites showed a high protein binding of $96.3 \%$ for abemaciclib, 93.4\% for M2, 96.8\% for M18 and 97.8\% for M20. The mean volume of distribution is 750 L. ${ }^{67,71}$ Abemaciclib is cleared mainly by hepatic metabolism, primarily by CYP3A4 with the formation of M2, M20 and M18 (Figure 2). The AUC of these metabolites represent $25 \%, 26 \%$ and $13 \%$, respectively, of the total circulating entities in plasma. ${ }^{67}$ In a mass balance study, abemaciclib was excreted as metabolites mainly in feces, with $81 \%$ of the administered dose recovered in feces, and $\approx 3 \%$ in urine. ${ }^{67,71}$

\section{Pharmacokinetics in special populations}

\section{Pediatric cancer patients}

Information on abemaciclib pharmacokinetics in the pediatric population is not available hitherto. ${ }^{72}$ Currently, two phase I studies are ongoing. ${ }^{70,71}$

\section{Patients with renal impairment}

No dedicated study has evaluated the effect of renal impairment on the pharmacokinetics of abemaciclib. However, a population pharmacokinetic analysis, including patients with normal renal function $(n=483)$, mild $(n=381)$ and moderate $(n=126)$ renal impairment, showed no significant differences in abemaciclib exposure. Therefore, no dose adjustment is required in patients with mild or moderate renal impairment. This was expected since the renal clearance of abemaciclib and its active metabolites is minor. The effect of severe renal impairment has not been determined yet. $64,67,71,75$

\section{Patients with hepatic impairment}

In a clinical trial, the total exposure of abemaciclib plus M2, M20 and M18 was similar in participants with mild and moderate hepatic impairment, showing an increase of $20 \%$ and $10 \%$, respectively, compared to participants with normal hepatic function. In contrast, severe hepatic impairment resulted in a 140\% increase in exposure of abemaciclib active entities. Furthermore, the mean plasma half-life of abemaciclib was prolonged $\left(\mathrm{t}_{1 / 2}=55 \mathrm{~h}\right.$ 
vs. $24 \mathrm{~h}$ in healthy subjects), absorption was slower ( $\mathrm{t}_{\max }=24 \mathrm{~h}$ vs. $7 \mathrm{~h}$ in healthy subjects) and protein binding decreased. Consequently, it is recommended to reduce the dose frequency to a once daily administration for patients with severe hepatic impairment (i.e. Child-Pugh class C). ${ }^{64,67,71,75}$

\section{Other factors influencing abemaciclib pharmacokinetics}

The influence of intrinsic factors on the abemaciclib pharmacokinetics was evaluated in a population pharmacokinetic analysis ( $n=994)$, in which sex, age, race and body weight were found to be insignificant covariates for the abemaciclib exposure. ${ }^{71,76}$ As a result, no special dose adjustments are required.

\section{Food effect}

An overview of food-effect studies for abemaciclib using a capsule or tablet formulation is provided in Table 3. The food-effect study with the tablet formulation is the most relevant since abemaciclib is marketed in this formulation. The exposure of abemaciclib increased with concomitant administration of a high-fat meal, but this was deemed not clinically relevant considering the high inter-subject variability and the fact that changes in exposure were within the abemaciclib therapeutic window. ${ }^{77,78}$ Therefore, abemaciclib can be administered with or without food.

\section{Drug-drug interactions}

Drug-drug interaction studies for abemaciclib are summarized in Table 4. The potential pharmacokinetic interaction between abemaciclib and fulvestrant or aromatase inhibitors was not formally evaluated. However, historical comparisons indicated that these drugs had no clinically relevant effect on the pharmacokinetics of abemaciclib, or vice versa. ${ }^{67,71,75}$

Due to the extensive metabolism of abemaciclib via CYP3A4, the exposure of abemaciclib plus its active metabolites M2, M20 and M18 is substantially affected when coadministered with strong CYP3A4 modulators.

Additionally, interactions with abemaciclib as a perpetrator could occur with substrates of transporters inhibited by abemaciclib (i.e. P-gp and renal transporters, Table 4).

\section{Pharmacokinetic-pharmacodynamic relationships}

\section{Exposure-response}

In a preclinical pharmacokinetic-pharmacodynamic model of xenograft tumors, $C_{\min } \geq 200$ $\mathrm{ng} / \mathrm{mL}$ has been identified as a potential efficacy threshold. Simulations with this model indicated that a maximum decrease in phosphorylated $\mathrm{Rb}$ levels was attained at a dose of $50 \mathrm{mg} / \mathrm{kg}$, corresponding to a $C_{\min }$ of $200 \mathrm{ng} / \mathrm{mL}$. A limitation of this study is that concentrations of the active metabolites $M 2$ and $M 20$ were not taken into account. ${ }^{79}$ 
In all three MONARCH-studies, exposure-response relationships were demonstrated. Although abemaciclib in the MONARCH-1 study ( $n=132$ ) could not be linked to objective response rate (ORR) and PFS, simulations with a pharmacokinetic-pharmacodynamic model found a positive relationship between exposure and tumor shrinkage. Also, these simulations suggested that the ORR would be higher at an abemaciclib dose of $200 \mathrm{mg}$ BID compared with 150 mg BID (31\% vs. 25\%, respectively). ${ }^{64}$ Using a similar approach, higher abemaciclib exposure was related to an increased tumor shrinkage in the MONARCH-2 study ( $n=477)$ as well, with the effect being most pronounced in the first months after start of treatment. ${ }^{64}$ Finally, in the MONARCH-3 study $(n=393)$ an exposureresponse relationship was not only established for tumor size reduction, but also for PFS. ${ }^{63}$

In summary, abemaciclib exposure was related to efficacy in several clinical trials. Therefore, it has been suggested that from an efficacy point of view, $200 \mathrm{mg}$ BID would be a better starting dose than $150 \mathrm{mg}$ BID. However, this higher starting dose is not deemed feasible, since $50 \%$ of patients needs a dose reduction due to toxicity. Based on the available data, no specific target for TDM can be proposed yet, but the optimal target might probably be somewhere between 169-197 ng/mL (i.e. the median exposure at 150 $\mathrm{mg}$ and $200 \mathrm{mg}$, respectively). Future exposure-response analyses need to identify the optimal threshold for efficacy, which could be performed using data from the MONARCHstudies or from a real-world patient cohort. Preferably, it should also be investigated whether it has additional value to include the concentrations of the active metabolites in this threshold, or that abemaciclib concentrations alone could serve as a proxy.

\section{Exposure-toxicity}

Higher abemaciclib concentrations were related to an increased incidence and severity of neutropenia. Dynamic pharmacokinetic-pharmacodynamic models for neutrophil count have been developed using data of the MONARCH-2 $(n=593)$ and MONARCH-3 $(n=477)$ studies. In these models, higher total $C_{\max }$ of abemaciclib and its active metabolites was related to a greater decrease in neutrophil production rate, and thus an increased risk of neutropenia. ${ }^{63,64}$

\section{Population pharmacokinetic models}

In a population pharmacokinetic model based on data obtained from the phase I study $(n=224)$, abemaciclib pharmacokinetics were best described by a linear one-compartment model with time- and dose-dependent bioavailability. Relative bioavailability decreased with an increasing dose, being 10\% lower at a dose of $200 \mathrm{mg}$ compared with $150 \mathrm{mg}$. This could be attributed to a saturable absorption, which is in line with preclinical data. ${ }^{79}$ Plasma exposure of abemaciclib also decreased over time with steady-state concentrations being attained after 70 days. ${ }^{76,79}$ 


\section{DISCUSSION}

By providing an overview of the clinical pharmacokinetics and pharmacodynamics of the three licensed CDK4/6 inhibitors palbociclib, ribociclib and abemaciclib, it becomes apparent that they share several characteristics. Similarities include the high interindividual variability in exposure, the predominant metabolism by CYP3A4, the brain penetration being limited by efflux transporters and the exposure-toxicity relationship for neutropenia. On the other hand, there are also substantial differences. First, abemaciclib has a divergent dosing schedule, as it is dosed twice daily and continuously, instead of once daily and intermittently for palbociclib and ribociclib. Second, dose-proportionality of pharmacokinetics varies between compounds. Palbociclib exposure increases linearly with increasing dose, whereas ribociclib exhibits a more than dose-proportional doseexposure relationship, and abemaciclib exposure, in contrast, increases less than proportionally with increasing dose, due to the lower fraction absorbed at higher doses. Third, abemaciclib has active and significantly abundant metabolites that should be taken into account when assessing its exposure (i.e. M2, M20 and M18), while this is not the case for palbociclib and ribociclib. Fourth, a clear exposure-efficacy relationship has been described for abemaciclib, while for palbociclib and ribociclib exposure-response analyses remain inconclusive. This might be explained by the applied methodologies and sample sizes that were used in these exposure-response analyses. It is important to further elucidate exposure-response relationship for all three CDK4/6 inhibitors. And finally, ribociclib frequently prolongs the QTc interval, in an exposure-related manner, whereas this, to our current knowledge, has not been reported for palbociclib and abemaciclib. These particular characteristics may support in selecting the most appropriate CDK4/6 inhibitor for individual patients.

Interestingly, the incidence of neutropenia is much lower for abemaciclib than for palbociclib and ribociclib. This is possibly caused by the greater selectivity of abemaciclib for CDK4 compared with CDK6, its twice daily dosing schedule, or the conversion to metabolites with less hematologic toxicity. ${ }^{59,62}$ In general, the effect of CDK4/6 inhibitors on neutrophil progenitor cells is cytostatic rather than cytotoxic, and associated with a notably low incidence of febrile neutropenia, in contrast to chemotherapy. ${ }^{37}$

Many patients require dose reductions due to neutropenia, which can remain problematic even at the lowest doses according to the label (i.e. $75 \mathrm{mg}$ QD for palbociclib, $200 \mathrm{mg}$ QD for ribociclib and $50 \mathrm{mg}$ BID for abemaciclib). If exposure in these patients is low, switching to an alternative treatment might be preferred, whereas in patients with adequate exposure prolonging the dose interval to every other day (QAD) for palbociclib and ribociclib, or QD for abemaciclib, could be an option, as has previously been described for pazopanib. ${ }^{80}$ Alternatively, the time off-treatment could be prolonged (i.e. 2-weeks-on/2- 
weeks-off treatment, as was allowed in the PALOMA-3 study). From a pharmacological point of view, though, prolonging the dose interval would be more rational.

Although it is known that CDK4/6 inhibitors combined with endocrine therapy provide an effective treatment strategy, it is currently unclear whether CDK4/6 inhibitors can best be added to first- or second-line treatment. This paramount question is currently being addressed in the SONIA study, a nationwide study in The Netherlands that will randomize 1000 patients between first- and second-line treatment with a CDK4/6 inhibitor. ${ }^{81}$ In an additional side study, pharmacokinetic samples are collected to further elucidate exposure-response relationships.

The currently approved CDK4/6 inhibitors are predominantly metabolized by CYP3A4. Therefore, increased exposure, and hence an increased risk of toxicity, can be expected in patients harboring mutations as in CYP3A4*22, as a result of lower levels of functional CYP3A4 and thus a decreased clearance. The reported prevalence of these mutations is up to $10 \%{ }^{82}$, and it could be argued that this subset of patients may benefit from a lower starting dose. This is currently being investigated in the STAR22 study. ${ }^{83}$

Although CDK4/6 inhibitors are currently only approved for the treatment of breast cancer, they are in clinical development for many other indications.

\section{CONCLUSIONS}

CDK4/6 inhibitors are a new class of promising oral targeted therapies in oncology, with complex pharmacokinetic and pharmacodynamic profiles, which we summarized in this review. Future studies should focus on the further exploration of exposure-response relationships and the potential for pharmacokinetically guided dose individualization. 
A
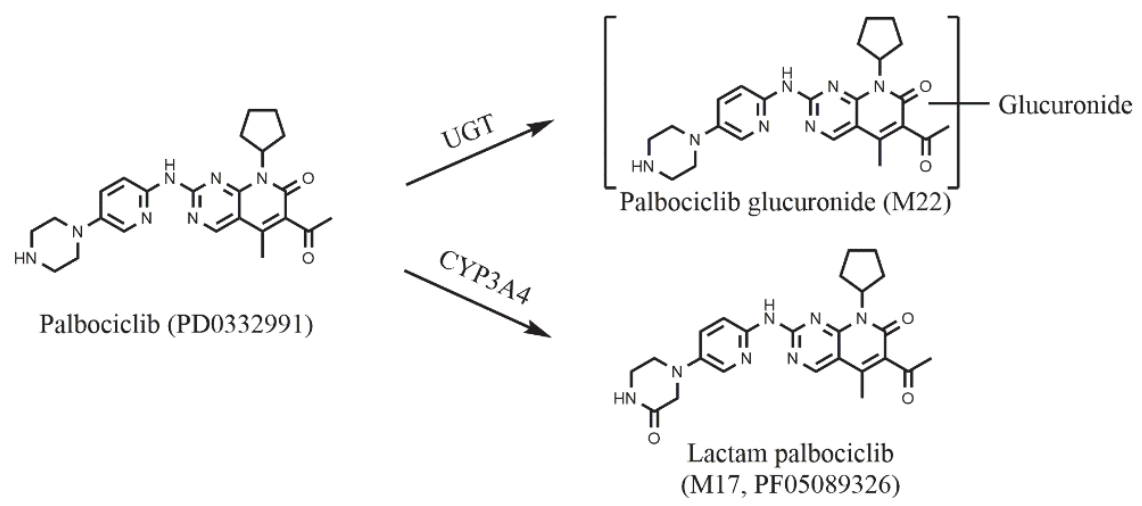

B
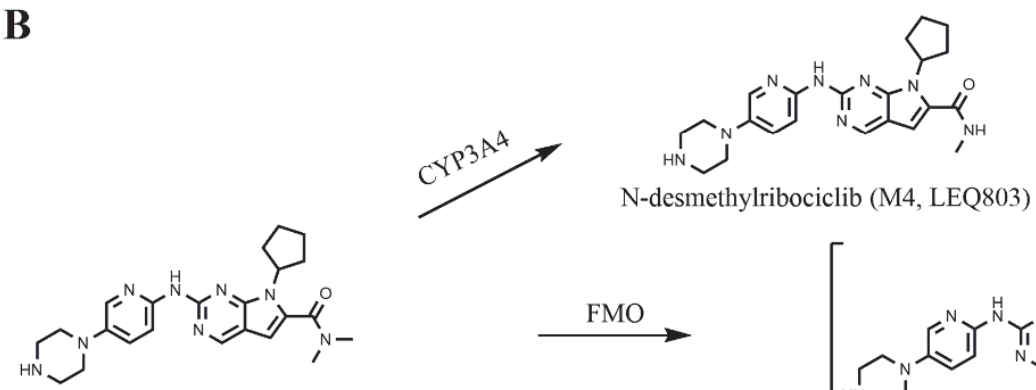

Ribociclib (LEE011)
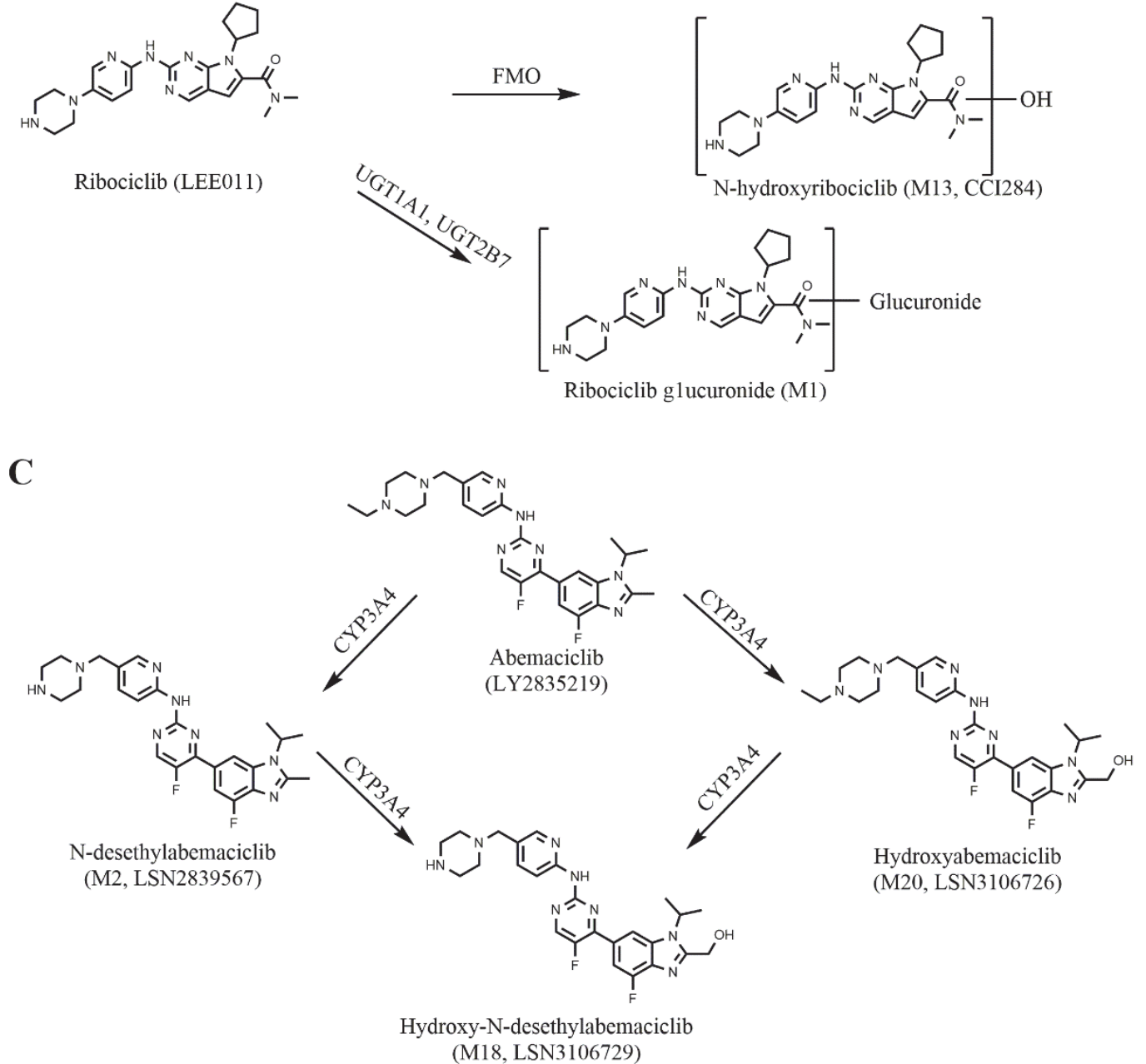

Figure 2 - Chemical structures of CDK4/6 inhibitors palbociclib (A), ribociclib (B) and abemaciclib (C) and their main metabolites

Chemical structures and metabolism were obtained from FDA and EMA reviews. ${ }^{9,46,75}$ This figure was created using ChemDraw Professional 15.0.

$U G T=$ Uridine 5'-diphospho-glucuronosyltransferase; $C Y P 3 A 4$ = cytochrome P450 3A4; FMO = Flavincontaining monooxygenase 


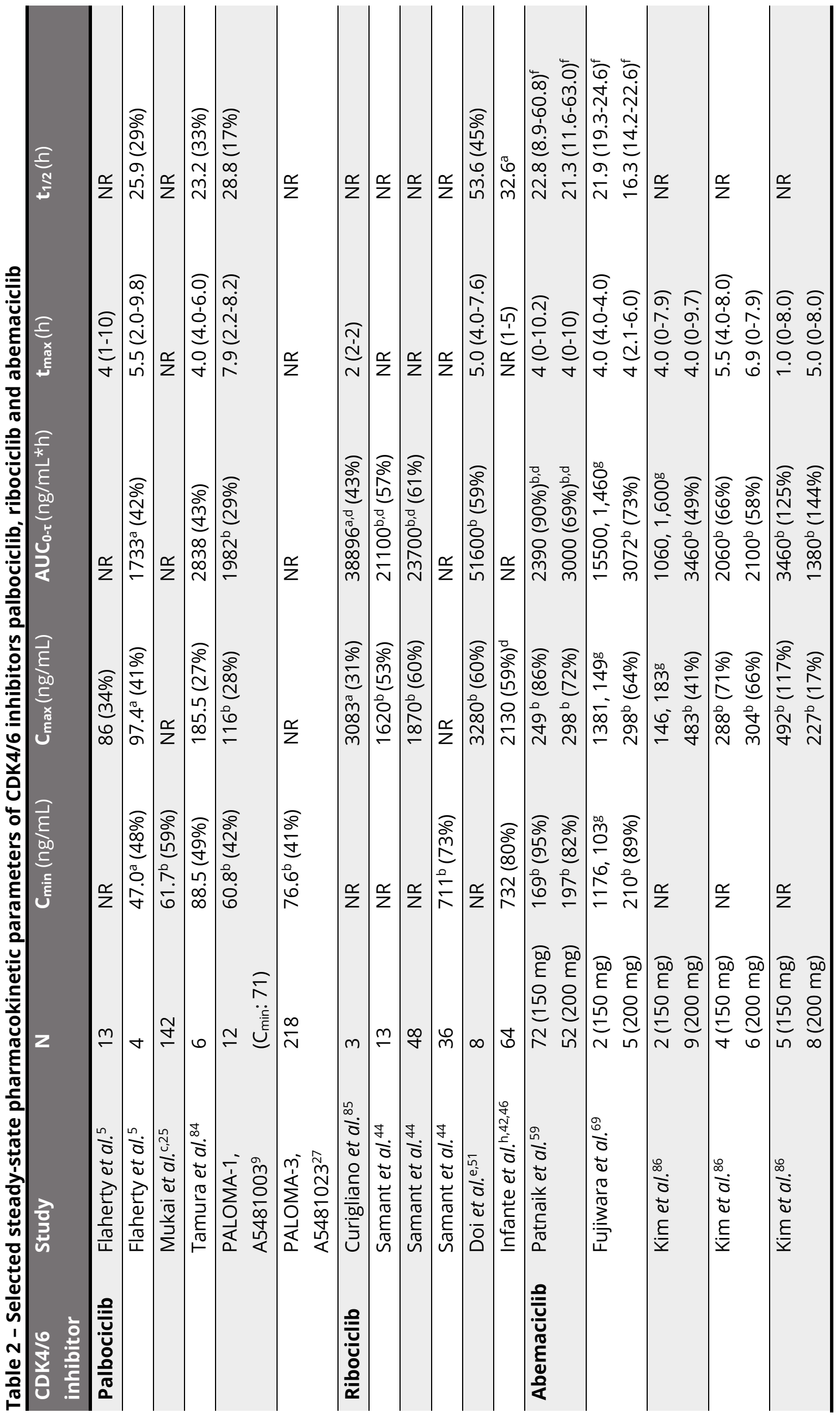




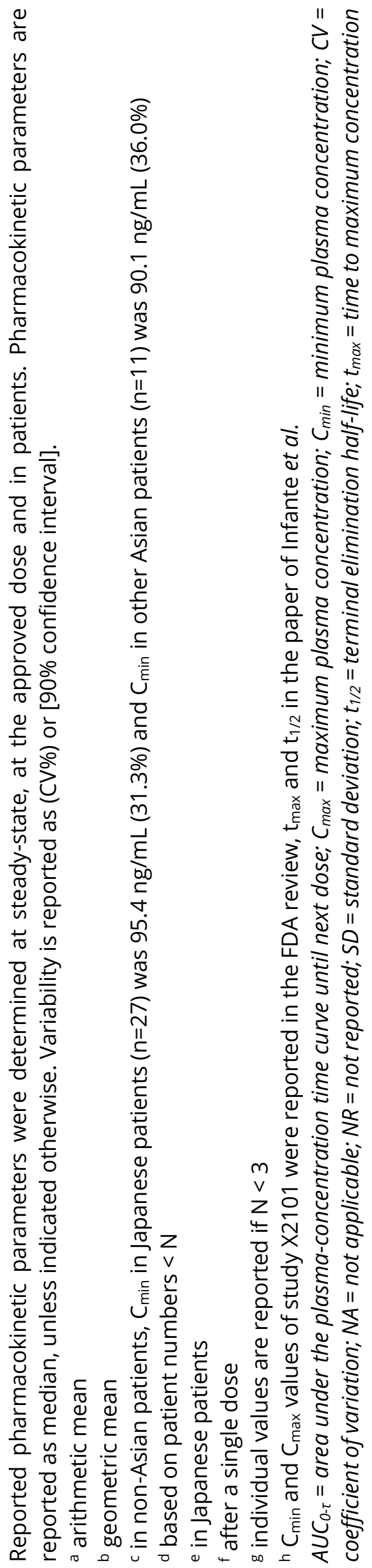




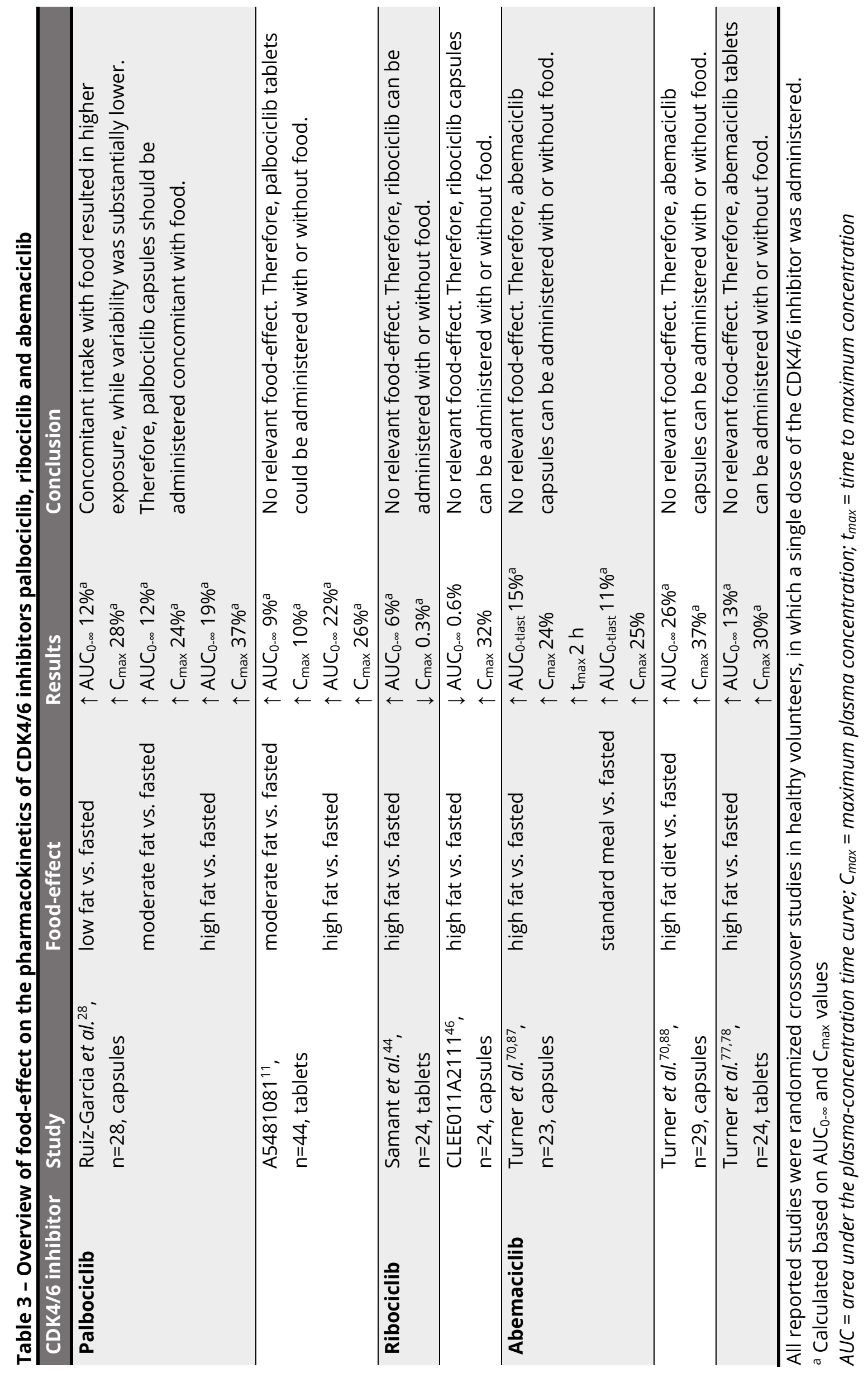




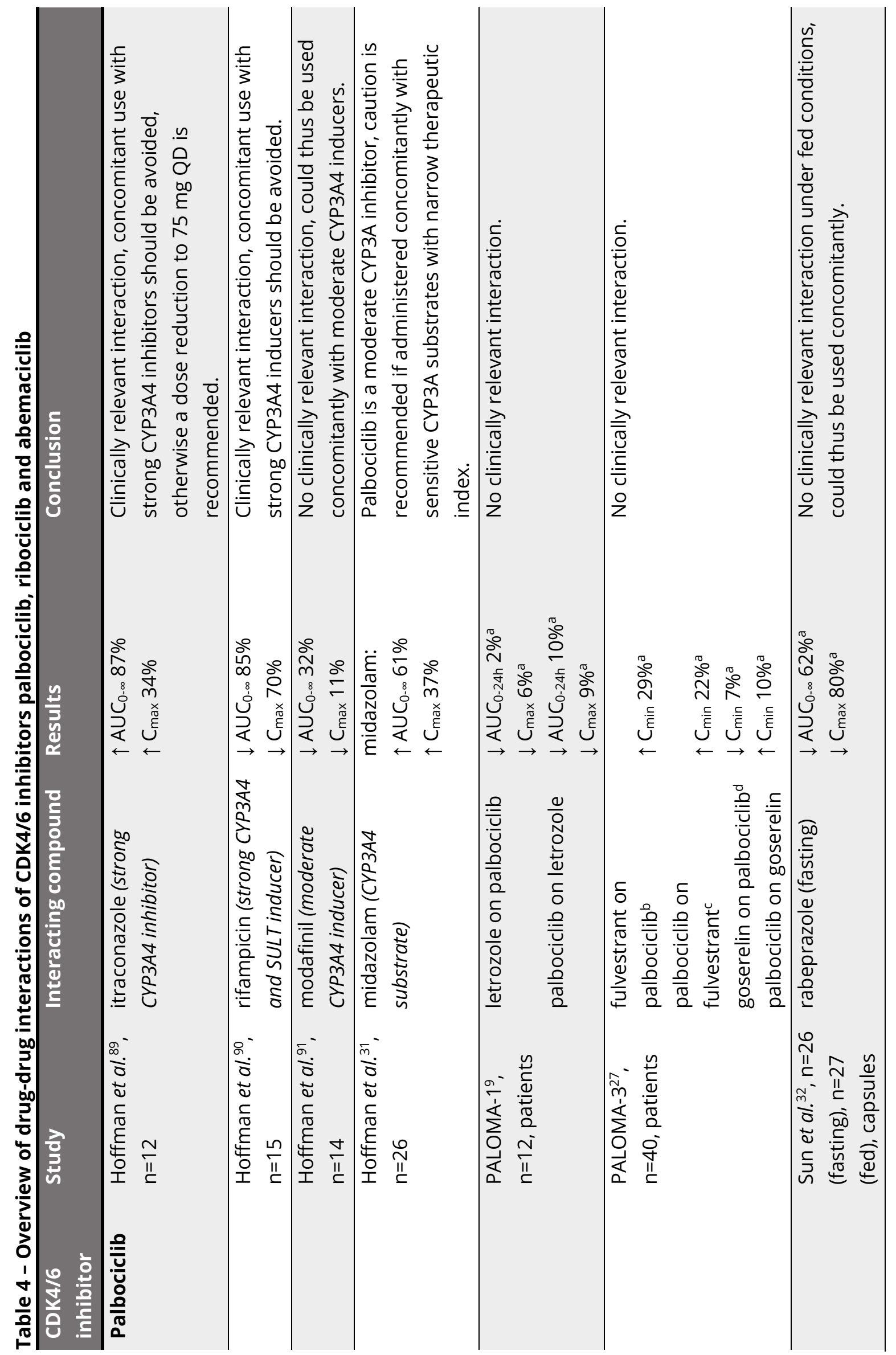




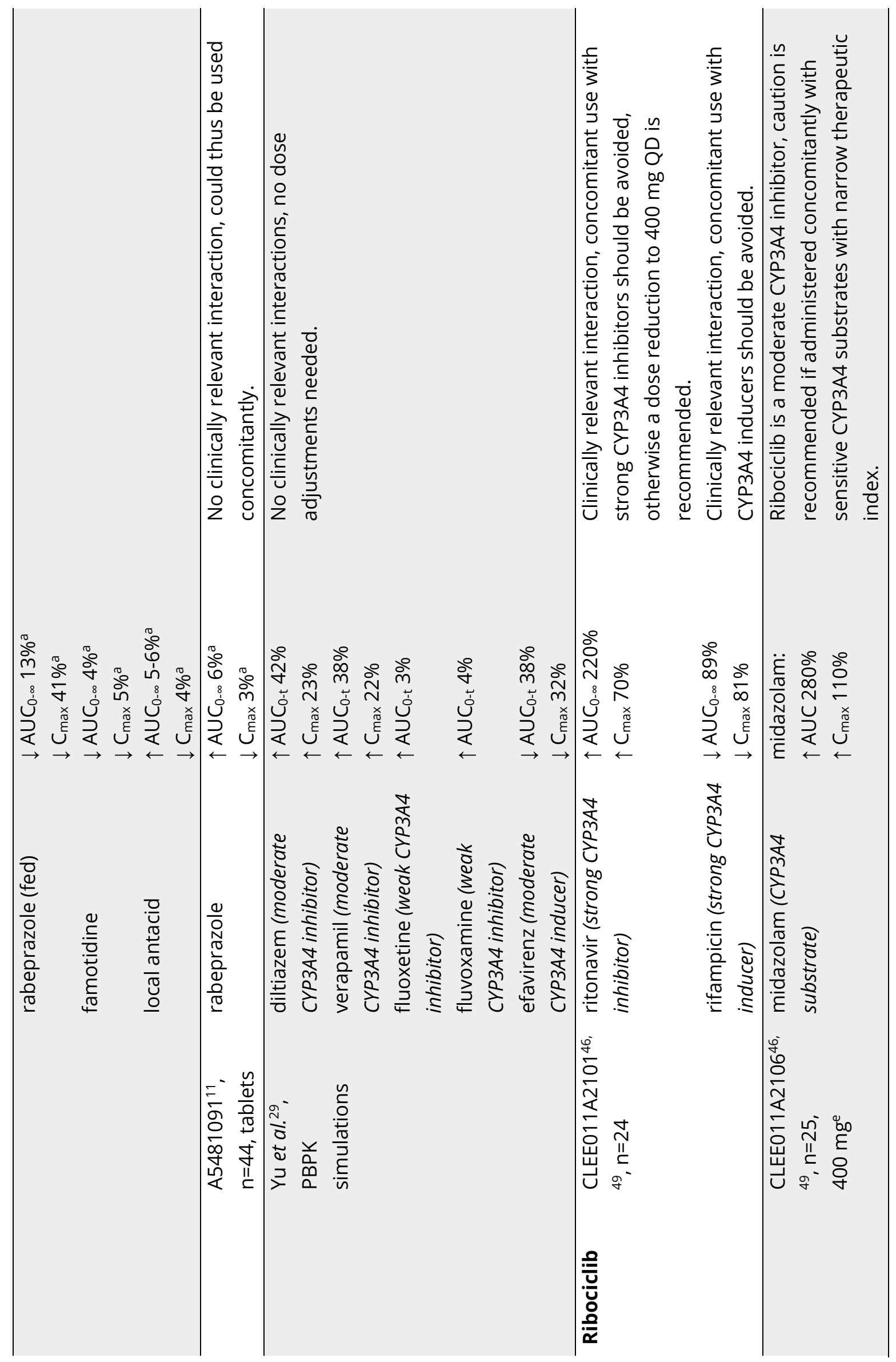




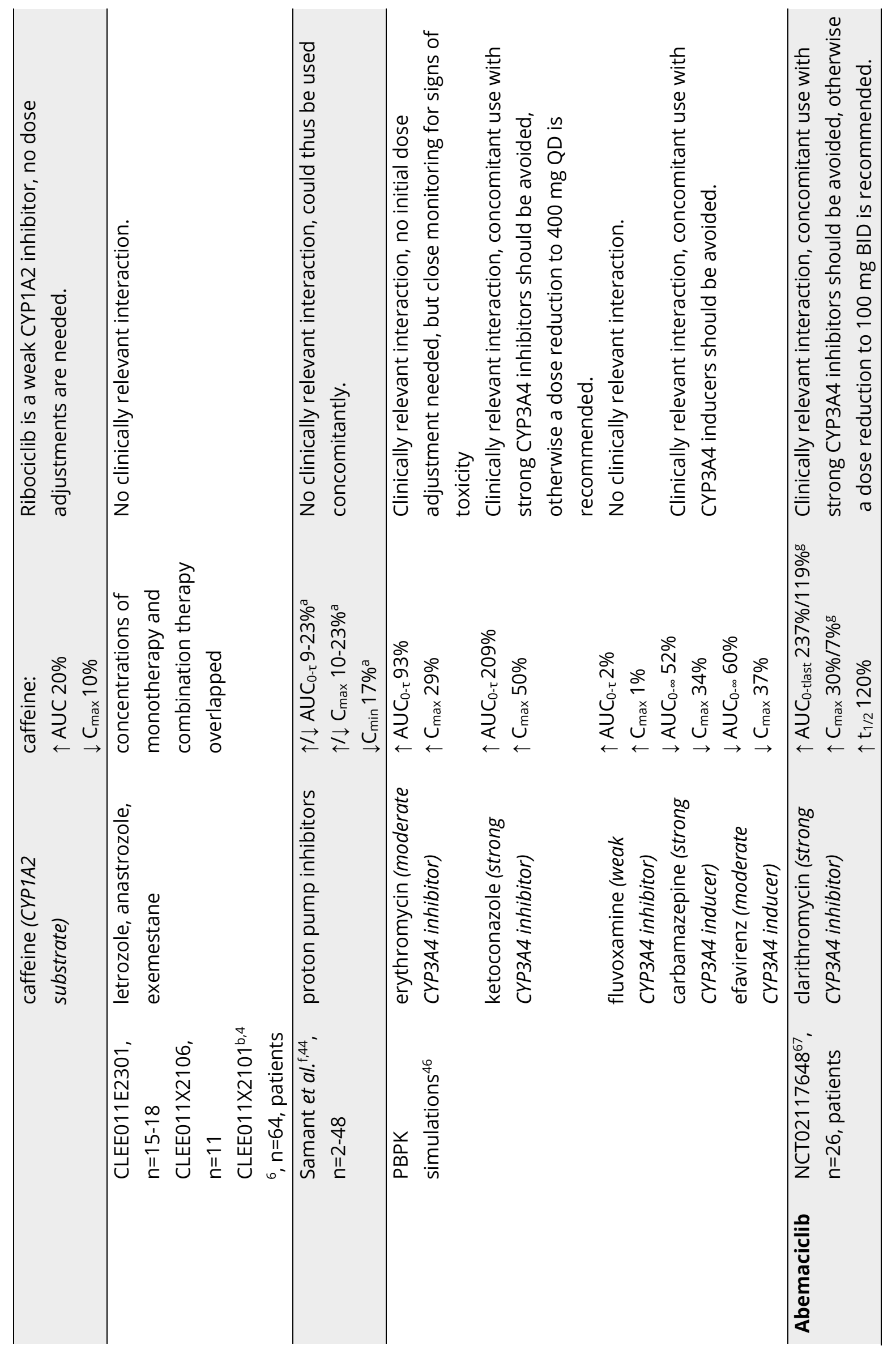




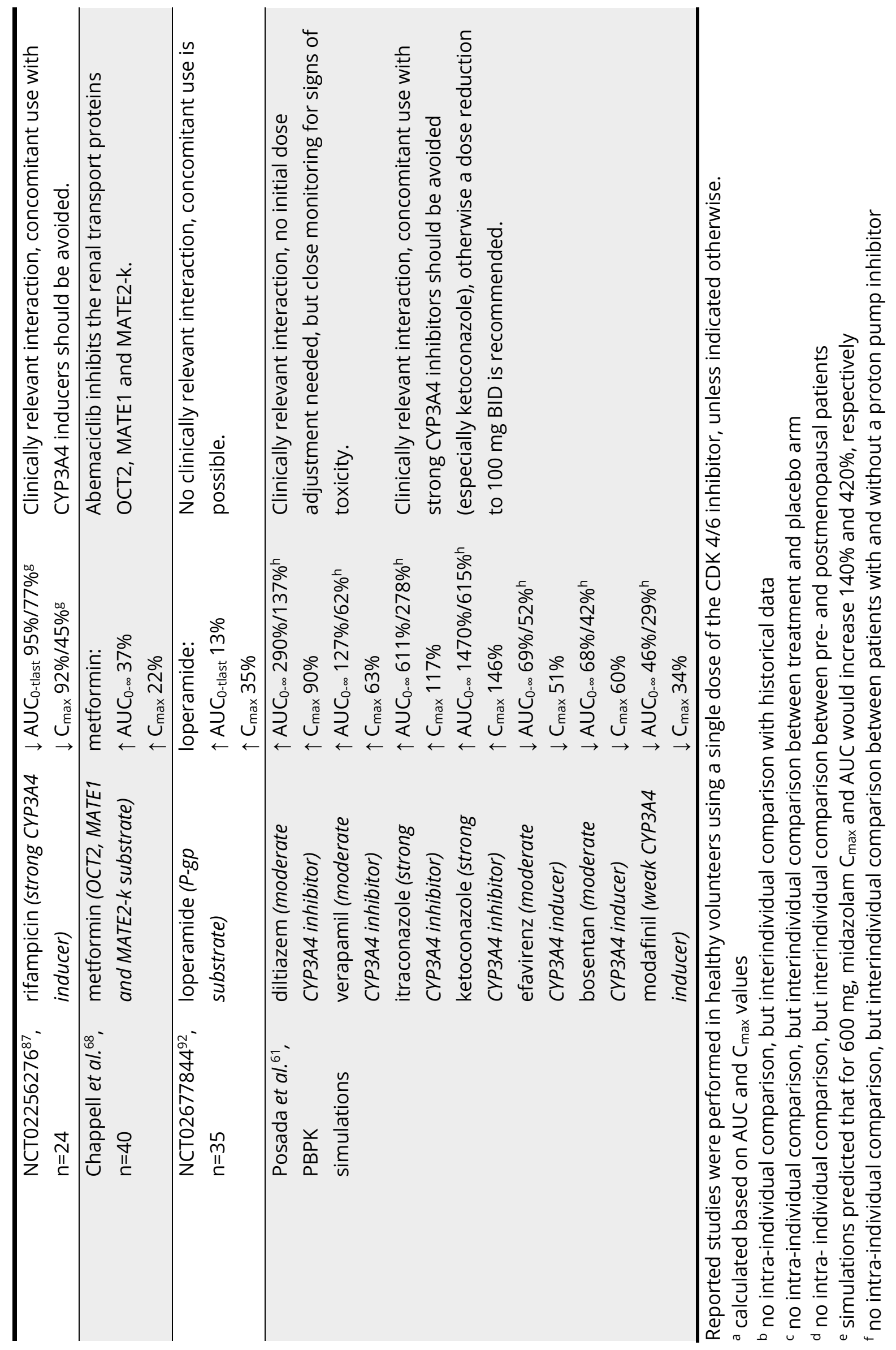




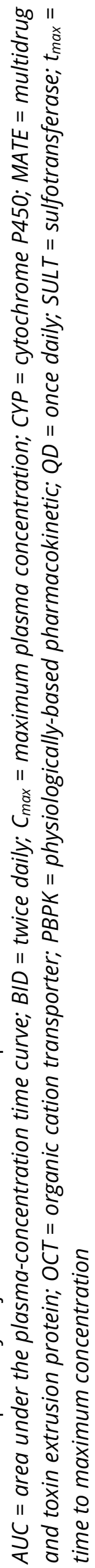




\section{REFERENCES}

1. Harbour JW, Luo RX, Dei Santi A, Postigo AA, Dean DC. Cdk phosphorylation triggers sequential intramolecular interactions that progressively block Rb functions as cells move through G1. Cell 1999; 98: 859-869.

2. Hall M, Peters G. Genetic alterations of cyclins, cyclin-dependent kinases, and Cdk Inhibitors in Human Cancer. Adv Cancer Res 1996; 68: 67-108.

3. Finn RS, Martin M, Rugo HS, Jones S, Im S-A, Gelmon K et al. Palbociclib and letrozole in advanced breast cancer. N Engl J Med 2016; 375: 1925-1936.

4. Turner NC, Ro J, André F, Loi S, Verma S, Iwata $\mathrm{H}$ et al. Palbociclib in hormone-receptorpositive advanced breast cancer. N EnglJ Med 2015; 373: 209-219.

5. Flaherty KT, LoRusso PM, DeMichele A, Abramson VG, Courtney R, Randolph SS et al. Phase I, dose-escalation trial of the oral cyclin-dependent kinase 4/6 inhibitor PD 0332991, administered using a 21-day schedule in patients with advanced cancer. Clin Cancer Res 2012; 18: 568-576.

6. Schwartz GK, LoRusso PM, Dickson MA, Randolph SS, Shaik MN, Wilner KD et al. Phase I study of PD 0332991, a cyclin-dependent kinase inhibitor, administered in 3-week cycles (schedule 2/1). Br J Cancer 2011; 104: 1862-1868.

7. Toogood PL, Harvey PJ, Repine JT, Sheehan DJ, VanderWel SN, Zhou H et al. Discovery of a potent and selective inhibitor of cyclin-dependent kinase 4/6. J Med Chem 2005; 48: 23882406.

8. Chen P, Lee N V., Hu W, Xu M, Ferre RA, Lam H et al. Spectrum and degree of CDK drug interactions predicts clinical performance. Mol Cancer Ther 2016; 15: 2273-2281.

9. Food and Drug Administration. Center for Drug Evaluation and Research. Clinical Pharmacology and Biopharmaceutics Review Palbociclib. 2014. Available from: https://www.accessdata.fda.gov/drugsatfda_docs/nda/2015/2071030rig1s000ClinPharmR. pdf.

10. Food and Drug Administration. Center for Drug Evaluation and Research. Waiver of in vivo bioavailability and bioequivalence studies for immediate-release solid oral dosage forms based on a biopharmaceutics classification system guidance for industry. 2017. Available from: https://www.fda.gov/media/70963/download.

11. Food and Drug Administration. Center for Drug Evaluation and Research. Clinical Pharmacology Review Palbociclib. 2019. Available from: https://www.accessdata.fda.gov/drugsatfda_docs/nda/2019/212436Orig1s000ClinPharmR. pdf.

12. Fry DW, Harvey PJ, Keller PR, Elliott WL, Meade MA, Trachet E et al. Specific inhibition of cyclin-dependent kinase 4/6 by PD 0332991 and associated antitumor activity in human tumor xenografts. Mol Cancer Ther 2004; 3: 1427-1437.

13. de Gooijer MC, Zhang P, Thota N, Mayayo-Peralta I, Buil LCM, Beijnen JH et al. P-glycoprotein and breast cancer resistance protein restrict the brain penetration of the CDK4/6 inhibitor palbociclib. Invest New Drugs 2015; 33: 1012-1019. 
14. Raub TJ, Wishart GN, Kulanthaivel P, Staton BA, Ajamie RT, Sawada GA et al. Brain exposure of two selective dual CDK4 and CDK6 inhibitors and the antitumor activity of CDK4 and CDK6 inhibition in combination with temozolomide in an intracranial glioblastoma xenograft. Drug Metab Dispos 2015; 43: 1360-1371.

15. Pabla N, Gibson AA, Buege M, Ong SS, Li L, Hu S et al. Mitigation of acute kidney injury by cell-cycle inhibitors that suppress both CDK4/6 and OCT2 functions. Proc Natl Acad Sci U S A 2015; 112: 5231-5236.

16. Chu X, Bleasby K, Chan GH, Nunes I, Evers R. The complexities of interpreting reversible elevated serum creatinine levels in drug development: does a correlation with inhibition of renal transporters exist? Drug Metab Dispos 2016; 44: 1498-1509.

17. Janssen JM, Dorlo TPC, Steeghs N, Beijnen JH, Hanff LM, van Eijkelenburg NKA et al. Pharmacokinetic targets for therapeutic drug monitoring of small molecule kinase inhibitors in pediatric oncology. Clin Pharmacol Ther. Online ahead of print 5 Feb 2020.

18. ClinicalTrials.gov. Palbociclib isethionate in treating younger patients with recurrent, progressive, or refractory central nervous system tumors. Available from: https://clinicaltrials.gov/ct2/show/NCT02255461.

19. ClinicalTrials.gov. Palbociclib and sorafenib, decitabine, or dexamethasone in treating patients with recurrent or refractory leukemia. Available from: https://clinicaltrials.gov/ct2/show/NCT03132454.

20. ClinicalTrials.gov. Study of palbociclib combined with chemotherapy in pediatric patients with recurrent/refractory solid tumors. Available from: https://clinicaltrials.gov/ct2/show/NCT03709680.

21. ClinicalTrials.gov. Palbociclib in combination with chemotherapy in treating children with relapsed acute lymphoblastic leukemia (ALL) or lymphoblastic lymphoma (LL). Available from: https://clinicaltrials.gov/ct2/show/NCT03792256.

22. ClinicalTrials.gov. Palbociclib in treating patients with relapsed or refractory $\mathrm{Rb}$ positive advanced solid tumors, non-hodgkin lymphoma, or histiocytic disorders with activating alterations in cell cycle genes (a pediatric MATCH treatment trial). Available from: https://clinicaltrials.gov/ct2/show/NCT03526250.

23. Food and Drug Administration. Center for Drug Evaluation and Research. Highlights of Prescribing Information Palbociclib. 2018. Available from: https://www.accessdata.fda.gov/drugsatfda_docs/label/2018/207103s007lbl.pdf.

24. Palmieri C, Macpherson I. Use of the Child-Pugh score in anticancer drug dosing decision making: proceed with caution. Lancet Oncol 2019; 20: e289.

25. Mukai H, Shimizu C, Masuda N, Ohtani S, Ohno S, Takahashi M et al. Palbociclib in combination with letrozole in patients with estrogen receptor-positive, human epidermal growth factor receptor 2-negative advanced breast cancer: PALOMA-2 subgroup analysis of Japanese patients. Int J Clin Oncol 2019; 24: 274-287.

26. Masuda N, Inoue K, Nakamura R, Rai Y, Mukai H, Ohno S et al. Palbociclib in combination with fulvestrant in patients with hormone receptor-positive, human epidermal growth factor receptor 2-negative advanced breast cancer: PALOMA-3 subgroup analysis of Japanese patients. Int J Clin Oncol 2019; 24: 262-273. 
27. Food and Drug Administration. Center for Drug Evaluation and Research. Clinical Pharmacology and Biopharmaceutics Review Palbociclib. 2016. Available from: https://www.accessdata.fda.gov/drugsatfda_docs/nda/2016/2071030rig1s002.pdf.

28. Ruiz-Garcia A, Plotka A, O'Gorman M, Wang DD. Effect of food on the bioavailability of palbociclib. Cancer Chemother Pharmacol 2017; 79: 1-7.

29. Yu Y, Loi C-M, Hoffman J, Wang D. Physiologically based pharmacokinetic modeling of palbociclib. J Clin Pharmacol 2017; 57: 173-184.

30. Netherlands Trial Register. Effect of moderate CYP3A4 inhibitor erythromycin on the pharmacokinetics of palbociclib. Available from: https://www.trialregister.nl/trial/7549.

31. Hoffman JT, Plotka A, O'Gorman M, Loi C-M, Kirkovsky L, Gallo-Stampino C et al. A phase 1 randomized, open-label, 2-sequence, 2-period crossover study of the effect of multiple doses of palbociclib (PD-0332991) on midazolam pharmacokinetics in healthy women of non-childbearing potential. [abstract]. In: Proceedings of the 105th Annual Meeting of the American Association for Cancer Research; 2014 Apr 5-9; San Diego, CA. Philadelphia (PA): AACR. Cancer Res. 2014; 74 (19 Suppl): Abstract nr CT419.

32. Sun W, Klamerus KJ, Yuhas LM, Pawlak S, Plotka A, O'Gorman M et al. Impact of acid-reducing agents on the pharmacokinetics of palbociclib, a weak base with pH-dependent solubility, with different food intake conditions. Clin Pharmacol Drug Dev 2017; 6: 614-626.

33. Sun W, Yu Y, Hoffman J, Turner NC, Cristofanilli M, Wang DD. Palbociclib exposure-response analyses in second-line treatment of hormone-receptor positive advanced breast cancer (ABC). J Clin Oncol 2017; 35: 1053-1053.

34. Food and Drug Administration. Center for Drug Evaluation and Research. Clinical Pharmacology Review Palbociclib. 2017. Available from: https://www.accessdata.fda.gov/drugsatfda_docs/nda/2019/2071030rig1s004.pdf.

35. Zheng J, Yu Y, Durairaj C, Amantea M, Dieras V, Finn R et al. Palbociclib exposure-response analyses in the treatment of hormone-receptor positive $(\mathrm{HR}+)$, human epidermal growth factor receptor 2 negative (HER2-) advanced breast cancer (ABC) [abstract]. In: Proceedings of the 2017 San Antonio Breast Cancer Symposium; Cancer Res 2018; 78: Abstract nr P5-2121.

36. Verheijen RB, $\mathrm{Yu} \mathrm{H}$, Schellens JHM, Beijnen JH, Steeghs N, Huitema ADR. Practical recommendations for therapeutic drug monitoring of kinase inhibitors in oncology. Clin Pharmacol Ther 2017; 102: 765-776.

37. Sun W, O'Dwyer PJ, Finn RS, Ruiz-Garcia A, Shapiro Gl, Schwartz GK et al. Characterization of neutropenia in advanced cancer patients following palbociclib treatment using a population pharmacokinetic-pharmacodynamic modeling and simulation approach. J Clin Pharmacol 2017; 57: 1159-1173.

38. Hortobagyi GN, Stemmer SM, Burris HA, Yap YS, Sonke GS, Paluch-Shimon S et al. Ribociclib as first-line therapy for HR-positive, advanced breast cancer. N Engl J Med 2016; 375: 17381748.

39. Hortobagyi GN, Stemmer SM, Burris HA, Yap YS, Sonke GS, Paluch-Shimon S et al. Updated results from MONALEESA-2, a phase III trial of first-line ribociclib plus letrozole versus placebo plus letrozole in hormone receptor-positive, HER2-negative advanced breast 
cancer. Ann Oncol 2018; 29: 1541-1547.

40. Slamon DJ, Neven P, Chia S, Fasching PA, De Laurentiis M, Im SA et al. Phase III randomized study of ribociclib and fulvestrant in hormone receptor-positive, human epidermal growth factor receptor 2-negative advanced breast cancer: MONALEESA-3. J Clin Oncol 2018; 36: 2465-2472.

41. Slamon DJ, Neven P, Chia S, Fasching PA, De Laurentiis M, Im SA et al. Overall survival with ribociclib plus fulvestrant in advanced breast cancer. N Eng/J Med 2020; 382: 514-524.

42. Infante JR, Cassier PA, Gerecitano JF, Witteveen PO, Chugh R, Ribrag V et al. A phase I study of the cyclin-dependent kinase 4/6 inhibitor ribociclib (LEE011) in patients with advanced solid tumors and lymphomas. Clin Cancer Res 2016; 22: 5696-5705.

43. Food and Drug Administration. Center for Drug Evaluation and Research. Chemistry Review Ribociclib. 2016. Available from: https://www.accessdata.fda.gov/drugsatfda_docs/nda/2017/2090920rig1s000ChemR.pdf.

44. Samant TS, Dhuria S, Lu Y, Laisney M, Yang S, Grandeury A et al. Ribociclib bioavailability is not affected by gastric $\mathrm{pH}$ changes or food intake: in silico and clinical evaluations. Clin Pharmacol Ther 2018; 104: 374-383.

45. Mitra A, Parrott N, Miller N, Lloyd R, Tistaert C, Heimbach T et al. Prediction of pH-dependent drug-drug interactions for basic drugs using physiologically based biopharmaceutics modeling: industry case studies. J Pharm Sci 2020; 109: 1380-1394.

46. Food and Drug Administration. Center for Drug Evaluation and Research. Clinical Pharmacology Review Ribociclib. 2017. Available from: https://www.accessdata.fda.gov/drugsatfda_docs/nda/2017/209092Orig1s000Multidiscipli neR.pdf.

47. Martínez-Chávez A, Van Hoppe S, Rosing H, Lebre MC, Tibben M, Beijnen JH et al. Pglycoprotein limits ribociclib brain exposure and cyp3a4 restricts its oral bioavailability. $\mathrm{Mol}$ Pharm 2019; 16: 3842-3852.

48. Sorf A, Hofman J, Kučera R, Staud F, Ceckova M. Ribociclib shows potential for pharmacokinetic drug-drug interactions being a substrate of $A B C B 1$ and potent inhibitor of $A B C B 1, A B C G 2$ and CYP450 isoforms in vitro. Biochem Pharmacol 2018; 154: 10-17.

49. Committee for Medicinal Products for Human Use. European Medicines Agency. European Public Assessment Report Ribociclib. 2017. Available from: https://www.ema.europa.eu/en/documents/assessment-report/kisqali-epar-publicassessment-report_en.pdf.

50. Wilson BE, Mok K, Kiely BE, Nguyen R, Moylan E. Association between ribociclib and changes in creatinine in patients with hormone receptor positive metastatic breast cancer. Intern Med J 2019; 49: 1438-1442.

51. Doi T, Hewes B, Kakizume T, Tajima T, Ishikawa N, Yamada Y. Phase I study of single-agent ribociclib in Japanese patients with advanced solid tumors. Cancer Sci 2018; 109: 193-198.

52. Food and Drug Administration. Center for Drug Evaluation and Research. Highlights of Prescribing Information Ribociclib. 2019. Available from: https://www.accessdata.fda.gov/drugsatfda_docs/label/2020/209092s003lbl.pdf. 
53. Geoerger B, Bourdeaut F, DuBois SG, Fischer M, Geller Jl, Gottardo NG et al. A phase I study of the CDK4/6 inhibitor ribociclib (LEE011) in pediatric patients with malignant rhabdoid tumors, neuroblastoma, and other solid tumors. Clin Cancer Res 2017; 23: 2433-2441.

54. ClinicalTrials.gov. Evaluation of renal function impairment on the pharmacokinetics of LEE011. Available from: https://clinicaltrials.gov/ct2/show/NCT02431481.

55. Committee for Medicinal Products for Human Use. European Medicines Agency. Summary of Product Characteristics Ribociclib. 2017. Available from: https://www.ema.europa.eu/en/documents/product-information/kisqali-epar-productinformation_en.pdf.

56. Food and Drug Administration. Center for Drug Evaluation and Research. Clinical Pharmacology Review Ribociclib. 2018. Available from: https://www.accessdata.fda.gov/drugsatfda_docs/nda/2019/209092Orig1s001.pdf.

57. Goetz MP, Toi M, Campone M, Trédan O, Bourayou N, Sohn J et al. MONARCH 3: abemaciclib as initial therapy for advanced breast cancer. J Clin Oncol 2017; 35: 3638-3646.

58. Sledge GW, Toi M, Neven P, Sohn J, Inoue K, Pivot $X$ et al. MONARCH 2: abemaciclib in combination with fulvestrant in women with $\mathrm{HR}+/ \mathrm{HER} 2$-advanced breast cancer who had progressed while receiving endocrine therapy. J Clin Oncol 2017; 35: 2875-2884.

59. Patnaik A, Rosen LS, Tolaney SM, Tolcher AW, Goldman JW, Gandhi L et al. Efficacy and safety of abemaciclib, an inhibitor of CDK4 and CDK6, for patients with breast cancer, non-small cell lung cancer, and other solid tumors. Cancer Discov 2016; 6: 740-753.

60. Food and Drug Administration. Center for Drug Evaluation and Research. Chemistry Review Abemaciclib. $2017 . \quad$ Available from: https://www.accessdata.fda.gov/drugsatfda_docs/nda/2017/2087160rig1s000ChemR.pdf.

61. Posada MM, Morse BL, Turner PK, Kulanthaivel P, Hall SD, Dickinson GL. Predicting clinical effects of CYP3A4 Modulators on Abemaciclib and Active Metabolites Exposure Using Physiologically Based Pharmacokinetic Modeling. J Clin Pharmacol 2020. doi:10.1002/jcph.1584.

62. Gelbert LM, Cai S, Lin X, Sanchez-Martinez C, Prado M Del, Lallena MJ et al. Preclinical characterization of the CDK4/6 inhibitor LY2835219: in-vivo cell cycledependent/independent anti-tumor activities alone/in combination with gemcitabine. Invest New Drugs 2014; 32: 825-837.

63. Food and Drug Administration. Center for Drug Evaluation and Research. Clinical Pharmacology Review Abemaciclib (in combination with aromatase inhibitors). 2017. Available from: https://www.accessdata.fda.gov/drugsatfda_docs/nda/2018/2088550rig1s000Multidiscipli neR.pdf.

64. Food and Drug Administration. Center for Drug Evaluation and Research. Clinical Pharmacology Review Abemaciclib (in combination with fulvestrant). 2017. Available from: https://www.accessdata.fda.gov/drugsatfda_docs/nda/2017/2087160rig1s000Multidiscipli neR.pdf.

65. Martínez-Chávez A, Tibben M, Lebre MC, Rosing $H$, Beijnen JH, Schinkel AH. The role of multidrug efflux transporters and CYP3A in the pharmacokinetics and tissue distribution of 
abemaciclib and its active metabolites. [abstract]. In: Proceedings of the 111th Annual Meeting of the American Association for Cancer Research; 2020 June 22-24. Philadelphia (PA): AACR; 2020. Abstract nr 2258.

66. Wu T, Chen Z, To KKW, Fang X, Wang F, Cheng B et al. Effect of abemaciclib (LY2835219) on enhancement of chemotherapeutic agents in $A B C B 1$ and $A B C G 2$ overexpressing cells in vitro and in vivo. Biochem Pharmacol 2017; 124: 29-42.

67. ClinicalTrials.gov. A study of LY2835219 in participants with cancer. Available from: https://clinicaltrials.gov/ct2/show/results/NCT02117648.

68. Chappell JC, Turner PK, Pak YA, Bacon J, Chiang AY, Royalty J et al. Abemaciclib inhibits renal tubular secretion without changing glomerular filtration rate. Clin Pharmacol Ther 2019; 105: 1187-1195.

69. Fujiwara Y, Tamura K, Kondo S, Tanabe Y, Iwasa S, Shimomura A et al. Phase 1 study of abemaciclib, an inhibitor of CDK 4 and 6 , as a single agent for Japanese patients with advanced cancer. Cancer Chemother Pharmacol 2016; 78: 281-288.

70. Turner K, Chappell J, Kulanthaivel P, Ng WT, Royalty J. Food effect on the pharmacokinetics of 200-mg abemaciclib in healthy subject. [abstract]. In: Proceedings of the 107th Annual Meeting of the American Association for Cancer Research; 2016 Apr 16-20; New Orleans, LA. Philadelphia (PA): AACR. Cancer Res 2016; 76(14 Suppl): Abstract nr CT152.

71. Committee for Medicinal Products for Human Use. European Medicines Agency. Summary of Product Characteristics Abemaciclib. 2017. Available from: https://www.ema.europa.eu/en/documents/product-information/verzenios-epar-productinformation_en.pdf.

72. Mills CC, Kolb EA, Sampson VB. Recent advances of cell-cycle inhibitor therapies for pediatric cancer. Cancer Res 2017; 77: 6489-6498.

73. ClinicalTrials.gov. Abemaciclib in children with DIPG or recurrent/refractory solid tumors (AflacST1501). Available from: https://clinicaltrials.gov/ct2/show/NCT02644460.

74. ClinicalTrials.gov. A study of abemaciclib (LY2835219) in combination with temozolomide and irinotecan and abemaciclib in combination with temozolomide in children and young adult participants with solid tumors. Available from: https://clinicaltrials.gov/ct2/show/NCT04238819.

75. Committee for Medicinal Products for Human Use. European Medicines Agency. Public Assessment Report Abemaciclib. 2018. Available from: https://www.ema.europa.eu/en/documents/assessment-report/verzenios-epar-publicassessment-report_en.pdf.

76. Tate SC, Sykes AK, Kulanthaivel P, Chan EM, Turner PK, Cronier DM. A Population pharmacokinetic and pharmacodynamic analysis of abemaciclib in a phase i clinical trial in cancer patients. Clin Pharmacokinet 2018; 57: 335-344.

77. Turner P, Chappell J, Aburub A, Ng W, Zhang W, Royalty J et al. Abemaciclib tablet formulation is bioequivalent to capsules [abstract]. In: Proceedings of the 2017 San Antonio Breast Cancer Symposium; 2017 Dec 5-9; San Antonio, TX. Philadelphia (PA): AACR. Cancer Res 2018; 78(4 Suppl): Abstract nr P1-10-14. 
78. ClinicalTrials.gov. A bioequivalence study comparing abemaciclib capsule and tablet formulations and effect of food on abemaciclib tablet pharmacokinetics in healthy subjects. Available from: https://clinicaltrials.gov/ct2/show/NCT02672423.

79. Tate SC, Cai S, Ajamie RT, Burke T, Beckmann RP, Chan EM et al. Semi-mechanistic pharmacokinetic/pharmacodynamic modeling of the antitumor activity of LY2835219, a new cyclin-dependent kinase 4/6 inhibitor, in mice bearing human tumor xenografts. Clin Cancer Res 2014; 20: 3763-3774.

80. Groenland SL, Katz D, Huitema ADR, Steeghs N. Harnessing soft tissue sarcoma with lowdose pazopanib - a matter of blood levels. BMC Cancer 2018; 18: 1-3.

81. van Ommen-Nijhof A, Konings IR, van Zeijl CJJ, Uyl-de Groot CA, van der Noort V, Jager A et al. Selecting the optimal position of CDK4/6 inhibitors in hormone receptor-positive advanced breast cancer - the SONIA study: study protocol for a randomized controlled trial. BMC Cancer 2018; 18: 1-7.

82. Zhou Y, Ingelman-Sundberg M, Lauschke VM. Worldwide distribution of cytochrome P450 alleles: a meta-analysis of population-scale sequencing projects. Clin Pharmacol Ther 2017; 102: 688-700.

83. Netherlands Trial Register. CYP3A4*22 genotype-guided dosing of TKIs in cancer patients: a new way of personalized therapy. Available from: https://www.trialregister.nl/trial/7514.

84. Tamura K, Mukai H, Naito $\mathrm{Y}$, Yonemori K, Kodaira M, Tanabe $\mathrm{Y}$ et al. Phase I study of palbociclib, a cyclin-dependent kinase 4/6 inhibitor, in Japanese patients. Cancer Sci 2016; 107: 755-763.

85. Curigliano G, Gómez Pardo P, Meric-Bernstam F, Conte P, Lolkema MP, Beck JT et al. Ribociclib plus letrozole in early breast cancer: a presurgical, window-of-opportunity study. Breast 2016; 28: 191-198.

86. Kim ES, Kelly K, Paz-Ares LG, Garrido P, Jalal S, Mahadevan D et al. Abemaciclib in combination with single-agent options in patients with stage IV non-small cell lung cancer: A phase Ib study. Clin Cancer Res 2018; 24: 5543-5551.

87. ClinicalTrials.gov. A study of LY2835219 in healthy participants. Available from: https://www.clinicaltrials.gov/ct2/show/NCT02256267.

88. ClinicalTrials.gov. A study of Abemaciclib (LY2835219) in healthy participants with and without food. Available from: https://clinicaltrials.gov/ct2/show/NCT02482935.

89. Hoffman JT, Loi C-M, O'Gorman M, Plotka A, Kosa M, Jakubowska A et al. A phase I openlabel, fixed-sequence, two-period crossover study of the effect of multiple doses of itraconazole on palbociclib (PD-0332991) pharmacokinetics in healthy volunteers. [abstract]. In: Proceedings of the 107th Annual Meeting of the American Association for Cancer Research; 2016 Apr 16-20; New Orleans, LA. Philadelphia (PA): AACR. Cancer Res 2016; 76 (14 Suppl): Abstract nr LB-196.

90. Hoffman JT, Plotka A, O'Gorman M, Chang A, Kosa M, Loi C-M et al. A phase 1 randomized, open-label, fixed-sequence, 2-period study of the effect of multiple doses of rifampin on palbociclib (PD-0332991) pharmacokinetics in healthy volunteers. [abstract]. In: Proceedings of the 106th Annual Meeting of the American Association for Cancer Research; 2015 Apr 18-22; Philadelphia, PA. Philadelphia (PA): AACR. Cancer Res 2015; 75 (15 Suppl): 
Abstract nr 4515.

91. Hoffman JT, Loi C-M, Plotka A, O'Gorman M, Shi H, Mori A et al. A phase I open-label fixedsequence two-period crossover study of the effect of multiple doses of modafinil on palbociclib (PD-0332991) pharmacokinetics in healthy volunteers. [abstract]. In: Proceedings of the 107th Annual Meeting of the American Association for Cancer Research; 2016 Apr 16-20; New Orleans, LA. Philadelphia (PA): AACR. Cancer Res 2016; 76 (14 Suppl): Abstract nr LB-198.

92. ClinicalTrials.gov. A study of abemaciclib in healthy participants (loperamide). Available from: https://clinicaltrials.gov/ct2/show/results/NCT02677844. 


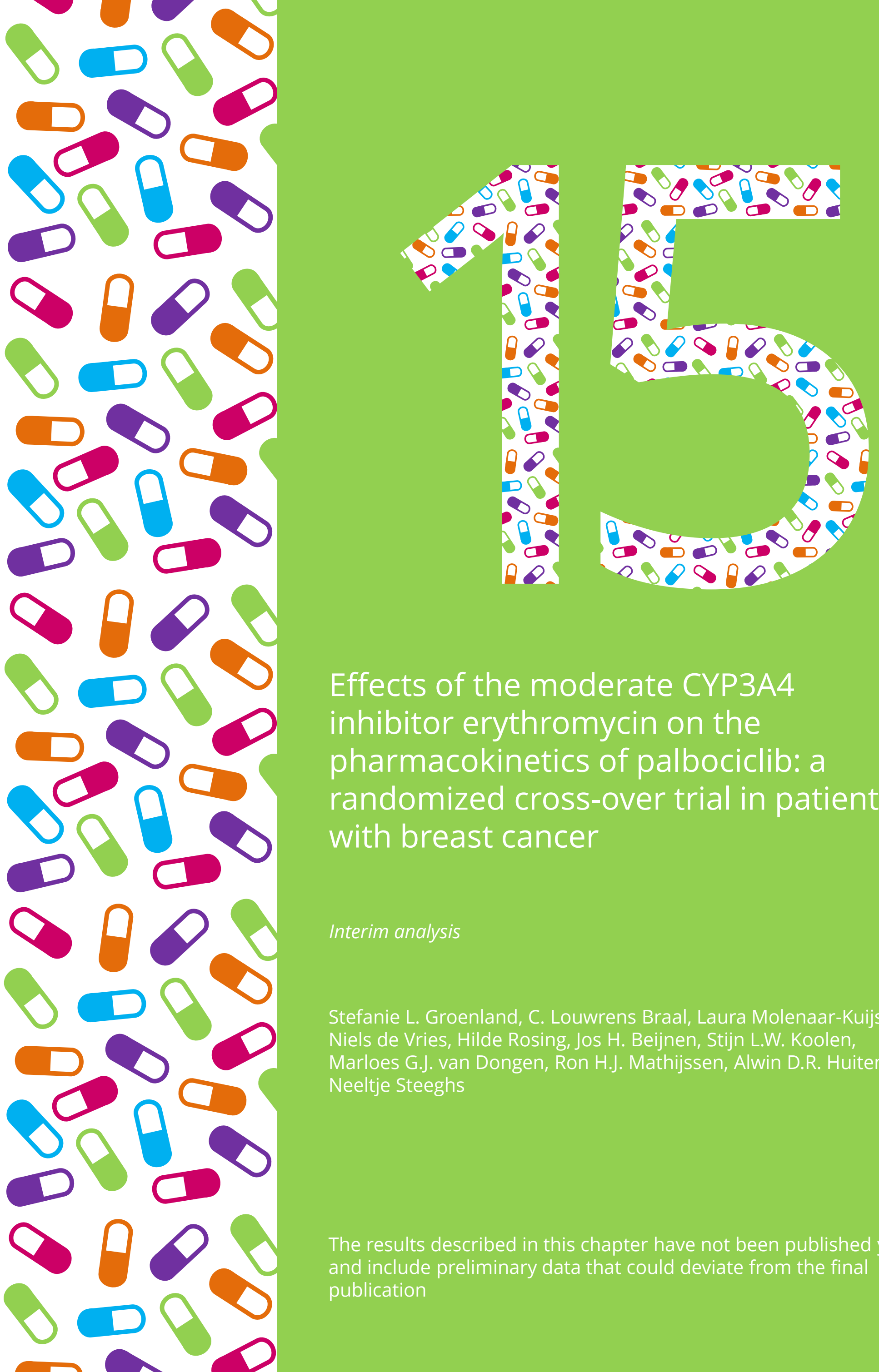

Effects of the moderate CYP3A4 inhibitor erythromycin on the pharmacokinetics of palbociclib: a randomized cross-over trial in patients with breast cancer

Interim analysis

Stefanie L. Groenland, C. Louwrens Braal, Laura Molenaar-Kuijsten, Niels de Vries, Hilde Rosing, Jos H. Beijnen, Stijn L.W. Koolen, Marloes G.J. van Dongen, Ron H.J. Mathijssen, Alwin D.R. Huitema, Neeltje Steeghs

The results described in this chapter have not been published yet and include preliminary data that could deviate from the final publication 


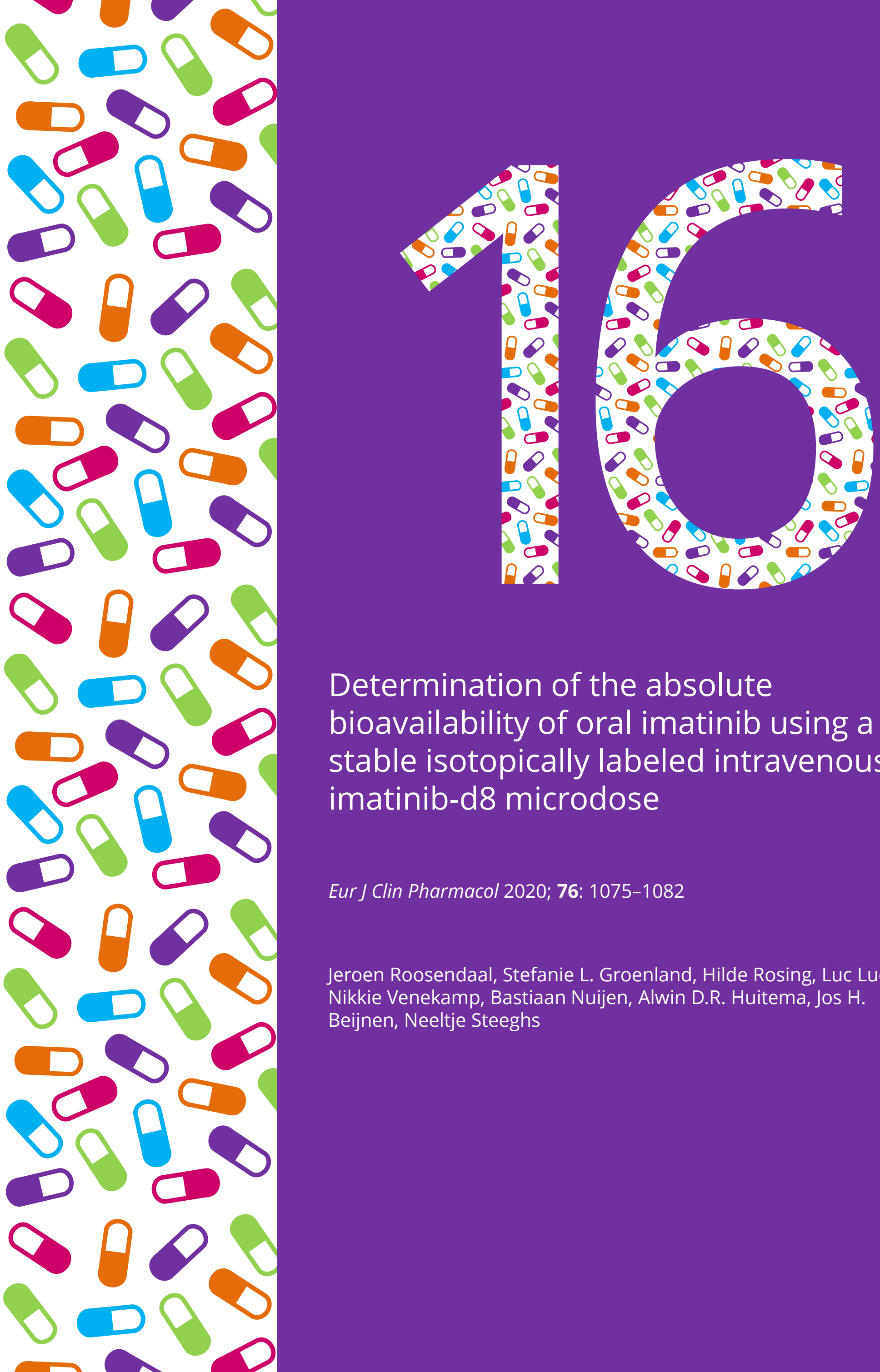

Determination of the absolute bioavailability of oral imatinib using a stable isotopically labeled intravenous imatinib-d8 microdose

Eur J Clin Pharmacol 2020; 76: 1075-1082

Jeroen Roosendaal, Stefanie L. Groenland, Hilde Rosing, Luc Lucas, Nikkie Venekamp, Bastiaan Nuijen, Alwin D.R. Huitema, Jos H. Beijnen, Neeltje Steeghs 


\section{ABSTRACT}

\section{Purpose}

The aim of this study was to ascertain whether the absolute bioavailability of oral imatinib (Glivec $®$ ) during steady state plasma pharmacokinetics in cancer patients could be determined through a concomitant intravenous administration of a single $100 \mu \mathrm{g}$ microdose of deuterium labeled imatinib (imatinib-d8). Secondly, the usefulness of liquid chromatography-tandem mass spectrometry (LC-MS/MS) was investigated for simultaneous analysis of orally and intravenously administered imatinib.

\section{Methods}

Included patients were on a stable daily dose of $400 \mathrm{mg}$ oral imatinib prior to study participation. On day 1, patients received a $100 \mu \mathrm{g}$ intravenous imatinib-d8 microdose $2.5 \mathrm{~h}$ after intake of the oral dose. Plasma samples were collected for $48 \mathrm{~h}$. Imatinib and imatinib-d8 concentrations were simultaneously quantified using a validated LC-MS/MS assay. The absolute bioavailability was calculated by comparing the dose-normalized exposure to unlabeled and stable isotopically labeled imatinib in plasma.

\section{Results}

A total of six patients were enrolled. All patients had a history of gastrointestinal stromal tumors (GIST). The median absolute bioavailability of oral imatinib at steady state was 76\% (range 44-106\%). Imatinib and imatinib-d8 plasma concentrations were quantified in all collected plasma samples, with no samples below the limit of quantification for imatinib-d8.

\section{Conclusion}

The absolute bioavailability of imatinib was successfully estimated at steady state plasma pharmacokinetics using the stable isotopically labeled microdose trial design. This study exhibits the use of a stable isotopically labeled intravenous microdose to determine the absolute bioavailability of an oral anticancer agent in patients with LC-MS/MS as the analytical tool. 


\section{INTRODUCTION}

The last decade has shown an increasing number of anticancer drugs that are administered orally. ${ }^{1-3}$ This so called "intravenous to oral switch" in oncology has resulted in an increased attention on the investigation of the absolute bioavailability during clinical drug development. Determining the absolute oral bioavailability of a new drug candidate facilitates the identification of potential developmental challenges such as absorption and first pass metabolism during the clinical development of a drug. Hence, the assessment of the absolute bioavailability is also crucial for the development of optimized oral formulations. As a result, data on the absolute bioavailability of novel oral drugs is now increasingly requested by the FDA and EMA., ${ }^{4,5}$

The conventional way to assess the absolute oral bioavailability is by using a two-period crossover study design, where an intravenous dose and an oral dose are administered to a study subject with a washout period in between. The absolute bioavailability is then calculated by dividing the plasma exposure after oral administration by the plasma exposure after intravenous administration. A limitation of this design is that for drugs that are poorly soluble in aqueous media, it might be impossible to develop an intravenous formulation at therapeutic strength. In addition, it assumes linear pharmacokinetics and constant clearance between the oral and intravenous dose event, which might not always be the case for drugs demonstrating a high intra-patient variability. This may result in a systemic error in the determination of the absolute bioavailability. ${ }^{6}$

A study design of co-administering an intravenous isotopically labeled microdose $(\leq 100$ $\mu \mathrm{g}$, less than $1 / 100^{\text {th }}$ of the therapeutic dose) with a therapeutic oral dose provides a solution to these problems. Because only a small amount of drug needs to be dissolved in an intravenous formulation, drug solubility issues can be circumvented. In addition, according to the current regulatory guidelines, clinical intravenous microdose studies could be carried out without additional toxicity investigations, saving costs, and time associated with intravenous drug development. ${ }^{7}$ Furthermore, because the intravenous microdose is administered during the same dose event as the oral therapeutic dose, the study duration is shortened and intra-occasion variability is not an issue, resulting in a more accurate determination of the absolute bioavailability and increased patients convenience.

Absolute bioavailability microdose trials can be performed using either radiolabeled or stable isotopically labeled drug processed into an intravenous formulation. In recent years, accelerator mass spectrometry (AMS) to measure a radiolabeled microdose has been utilized to support several clinical absolute bioavailability studies. ${ }^{8}$ A drawback of AMS is that sample analysis is labor and time intensive, expensive, and that AMS is only available in a limited number of places dedicated to biomedical research worldwide. ${ }^{9}$ An alternative analytical approach for conducting microdose studies is using liquid 
chromatography coupled to tandem mass spectrometry (LC-MS/MS) to quantitate both the intravenous and the oral drug. Because both labeled and unlabeled drug can be measured simultaneously with LC-MS/MS, it is an elegant and cost-effective alternative to AMS. ${ }^{9,10}$

For the group of tyrosine kinase inhibitors, an important class of novel oral anticancer agents, it has been demonstrated that for many drugs registered in the past years, the absolute bioavailability has not been assessed the time of drug licensing. ${ }^{3}$ One reason for this might be that poor drug solubility hampers the development of an aqueous intravenous dose at therapeutic strength, making it almost impossible to use the conventional crossover trial design. In this trial, we used imatinib, a tyrosine kinase inhibitor used for the treatment of chronic myeloid leukemia (CML) and gastrointestinal stromal tumors (GIST), to demonstrate the potential of using a stable isotopically labeled $100 \mu \mathrm{g}$ microdose in combination with LC-MS/MS to assess the absolute bioavailability.

The objective of this study was to ascertain whether the absolute bioavailability of oral imatinib (Glivec ${ }^{\circledR}$ ) during steady state plasma pharmacokinetics in cancer patients could be determined through a concomitant intravenous administration of a single $100 \mu \mathrm{g}$ microdose of deuterium labeled imatinib (imatinib-d8). Secondly, the usefulness of liquid chromatography-tandem mass spectrometry (LC-MS/MS) is investigated for simultaneous analysis of orally and intravenously administered imatinib.

\section{MATERIALS AND METHODS}

\section{Study design and sample collection}

This was a single center, open-label study in which the absolute bioavailability of imatinib (Figure 1A) was determined at steady state by concomitant administration of an intravenous microdose of stable isotopically labeled imatinib-d8 (Figure 1B). Figure 2 provides a schematic overview of the study design. On day 1 , patients received a single intravenous microdose of imatinib-d8, next to the standard treatment of imatinib $400 \mathrm{mg}$ once daily (Glivec ${ }^{\circledR}$ ). After intake of imatinib at approximately 08:30 a.m., a $100 \mu \mathrm{g}$ imatinib-d8 microdose was administered intravenously as a bolus injection at the estimated maximum plasma concentration $\left(t_{\max }\right)$ of oral imatinib ( $2.5 \mathrm{~h}$ post oral dose). Oral imatinib intake was not interrupted for the duration of the study. The study (Netherlands Trial Register, NTR7642, www.nederlandstrialregister.nl) was approved by both the Medical Ethics Committee of The Netherlands Cancer Institute, Amsterdam, The Netherlands, as well as the competent authority (Centrale Commissie Mensgebonden Onderzoek, CCMO). The study was conducted in accordance with the Declaration of Helsinki. All participants provided written informed consent prior to study assessments. 
A<smiles>Cc1ccc(NC(=O)c2ccc(CN3CCN(C)CC3)cc2)cc1Nc1nccc(-c2cccnc2)n1</smiles>

B<smiles>[2H]C1([2H])N(C)C([2H])([2H])C([2H])([2H])N(Cc2ccc(C(=O)Nc3ccc(C)c(Nc4nccc(-c5cccnc5)n4)c3)cc2)C1([2H])[2H]</smiles>

Figure 1 - Molecular structures

A: imatinib

B: imatinib-d8

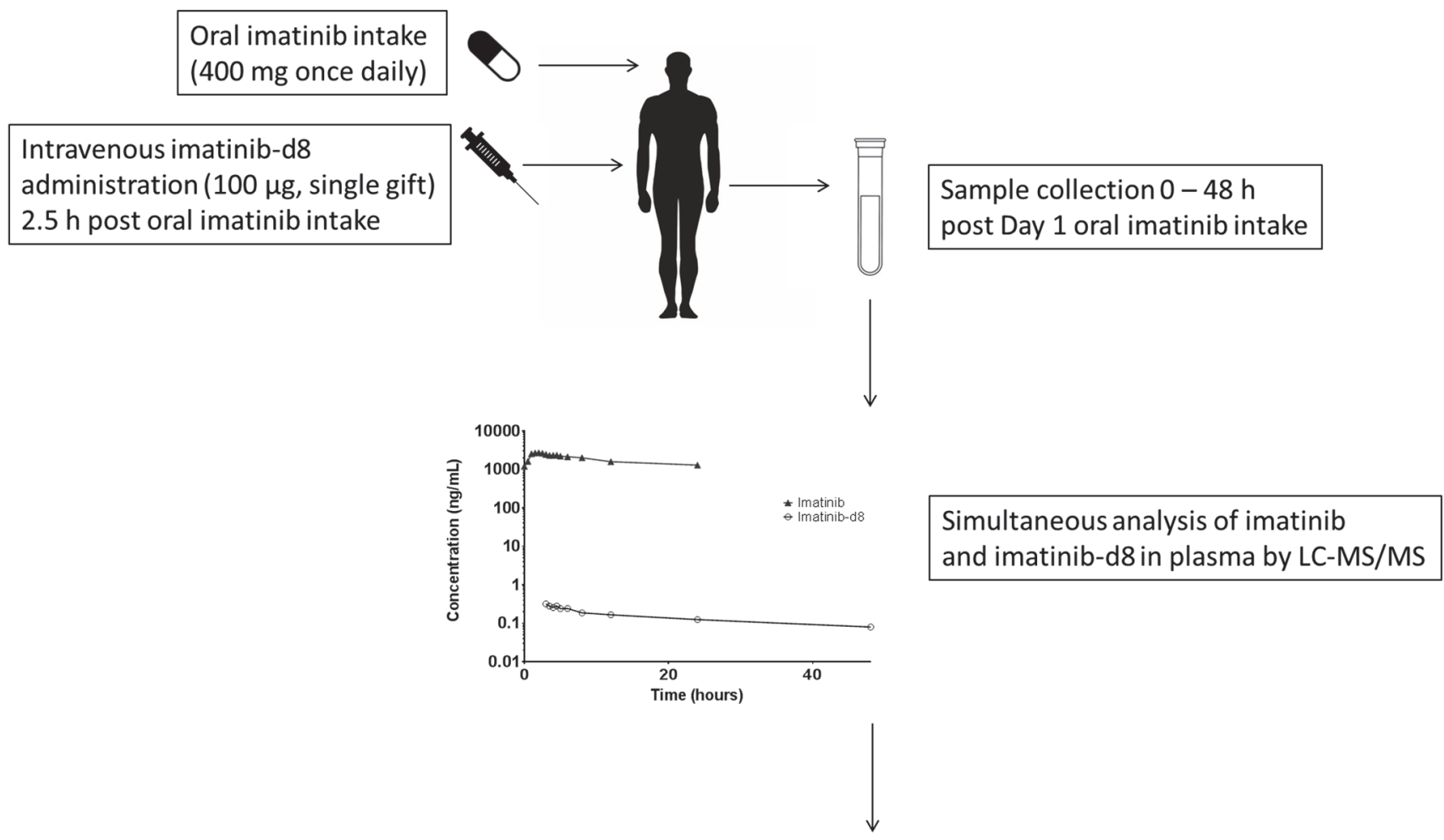

AUC calculation for absolute bioavailability determination Imatinib: $\mathrm{AUC}_{0-24 \mathrm{~h}}$ (steady state plasma pharmacokinetics) Imatinib-d8: $A \cup C_{0 \text {-inf }}($ single gift)

Figure 2 - Schematic overview of the imatinib absolute bioavailability microdose trial design $L C-M S / M S=$ liquid chromatography coupled to tandem mass spectrometry; $A U C_{0-24 h}=$ area under the plasma concentration-time curve up to 24 hours; $A \cup C_{0 \text {-inf }}=$ area under the plasma concentration-time curve extrapolated to infinity 


\section{Patients}

Patients $\geq 18$ years of age, treated with imatinib $400 \mathrm{mg}$ once daily in the morning for at least 7 days (steady state plasma concentrations), were included. Subjects needed to have acceptable organ function, as evidenced by laboratory data: aspartate aminotransferase (ASAT) and alanine aminotransferase (ALAT) $\leq 5 x$ the upper limit of normal (ULN), total serum bilirubin $\leq 2 x$ ULN, renal function as defined by glomerular filtration rate (GFR $M D R D)>40 \mathrm{~mL} / \mathrm{min} / 1.73 \mathrm{~m}^{2}$. Subjects who received treatment with inhibitors or inducers of CYP3A4 were excluded.

\section{Treatment}

Patients received $400 \mathrm{mg}$ imatinib (Glivec $®$ ) tablets once daily in the morning as part of routine clinical care. According to the drug label, imatinib was ingested concomitant with a meal. ${ }^{11}$ Meals were not standardized. The reference drug imatinib-d8 (Toronto Research Chemicals, ON, Canada) was formulated in the hospital pharmacy of The Netherlands Cancer Institute and was supplied as a $0.1 \mathrm{mg} / \mathrm{mL}$ in $\mathrm{NaCl} 0.9 \%$ solution for intravenous injection.

\section{Sample collection, processing, and analysis}

From day 1 to day 3, pharmacokinetic sampling was performed. Blood samples were collected at predose, 0.5, 1, 1.5, 2, 2.5 (pre intravenous microdose), 3, 3.5, 4, 4.5, 5, 6, 8, 12, 24 (pre day 2 oral dose) and 48 h (pre day 3 oral dose), after oral imatinib intake.

Peripheral blood for quantification of imatinib and imatinib-d8 was drawn in 4-mL $K_{2}$ EDTA tubes and centrifuged directly after collection ( $\left.1500 \mathrm{~g}, 10 \mathrm{~min}, 4^{\circ} \mathrm{C}\right)$. Plasma was stored at $-80{ }^{\circ} \mathrm{C}$ until analysis. A validated LC-MS/MS assay was used for the simultaneous quantification of imatinib and imatinib-d8. ${ }^{12}$ Routine sample analysis acceptance criteria for bioanalytical data according to FDA and EMA guidelines ${ }^{13,14}$ were applied and results were reported using Analyst 1.6.2. software (Sciex, Framingham, MA, USA).

\section{Pharmacokinetic analysis and absolute bioavailability calculation}

Imatinib and imatinib-d8 plasma concentrations were used to determine the maximum observed plasma concentration $\left(C_{\max }\right)$, time to reach maximum plasma concentration $\left(t_{\max }\right)$, area under the plasma concentration-time curve from time zero to $24 \mathrm{~h}\left(\mathrm{AUC}_{0-24 \mathrm{~h}}\right)$ for imatinib, and from time zero to infinity ( $A \cup C_{0-\text { inf }}$ ) for imatinib-d8, the terminal phase half-life $\left(\mathrm{t}_{1 / 2}\right)$ and the elimination rate constant from the central compartment $\left(k_{\mathrm{e}}\right)$, the volume of distribution $\left(\mathrm{V}_{\mathrm{d}}\right)$, and total plasma clearance $(\mathrm{CL})$. Parameters were calculated using plasma concentration-time curves obtained from 0 to $24 \mathrm{~h}$ for imatinib, and from 0 to $48 \mathrm{~h}$ for imatinib-d8. Non-compartmental analysis was performed using $\mathrm{R}$ version $3.0 .1 .^{15}$

As the exposure at steady state plasma pharmacokinetics during the dose interval is equivalent to the exposure from zero to infinity following a single administration ${ }^{16}$, the 
$A \cup C_{0-24 h}$ for imatinib and the $A \cup C_{0-\text { inf }}$ for imatinib-d8 could be used to calculate the absolute bioavailability without dose interruptions for the patients.

The absolute bioavailability (F) of oral imatinib was calculated as the ratio of dosenormalized exposures of the oral (po) imatinib and intravenous (iv) imatinib-d8 gift expressed as a percentage using the following formula:

$$
F(\%)=\frac{\left[A U C_{0-24}\right]_{p o} / \text { Dose }_{p o}}{\left[A U C_{0-i n f}\right]_{i v} / \text { Dose }_{i v}} \times 100
$$

\section{RESULTS}

A total of six patients have been included, with a median age of 65 years (range 52-72). Of these patients, 50\% received adjuvant imatinib treatment for GIST and 50\% were treated in the metastatic setting. An overview of patient baseline characteristics can be found in

\section{Table 1.}

Table 1 - Patient baseline characteristics

\begin{tabular}{|c|c|}
\hline Characteristic & Patients \\
\hline Age, years & $65(52-72)$ \\
\hline Gender, male & $4(67 \%)$ \\
\hline \multicolumn{2}{|l|}{ Tumor type } \\
\hline GIST & $6(100 \%)$ \\
\hline \multicolumn{2}{|l|}{ Treatment setting } \\
\hline Adjuvant & $3(50 \%)$ \\
\hline Metastatic & $3(50 \%)$ \\
\hline \multicolumn{2}{|l|}{ Previous surgery type } \\
\hline Wedge partial resection of the stomach & $3(50 \%)$ \\
\hline Partial small bowel resection & $1(17 \%)$ \\
\hline Multiple resections ${ }^{a}$ & $2(33 \%)$ \\
\hline Time on imatinib treatment (in years) & $3.2(0.3-13.0)$ \\
\hline Albumin (in g/L) & $44(42-47)$ \\
\hline eGFR $^{\mathbf{b}}$ (in $\mathrm{mL} / \mathrm{min}$ ) & $69(58-84)$ \\
\hline
\end{tabular}

Data are expressed as no. (\%) or median (range), as appropriate.

a one patient with wedge partial resection of the stomach and partial colon resection, one patient with wedge partial resection of the stomach, splenectomy and partial pancreas resection

${ }^{b}$ eGFR was calculated using the MDRD-4 formula

eGFR = estimated glomerular filtration rate; GIST = gastrointestinal stromal tumour

All included patients were evaluable for pharmacokinetic analysis. Mean plasma concentration-time curves of imatinib and imatinib-d8 can be found in Figure 3. A summary of imatinib and imatinib-d8 pharmacokinetic parameters can be found in Table 2. 
The absorption of imatinib after oral administration of tablets was rapid, with a median $\mathrm{t}_{\max }$ of $2 \mathrm{~h}$. The $\mathrm{C}_{\max }$ of oral imatinib at steady state was $2.9 \pm 0.8 \mu \mathrm{g} / \mathrm{mL}$. The mean $A \cup \mathrm{C}_{0}$ ${ }_{24}$ for oral imatinib was $42.6 \pm 12.9 \mu \mathrm{g} \cdot \mathrm{h} / \mathrm{mL}$, and the mean $A \cup C_{0 \text {-inf }}$ for imatinib-d8 was $0.015 \pm 0.007 \mu \mathrm{g} \cdot \mathrm{h} / \mathrm{mL}$. The $A U_{C_{0} \text {-inf }}$ for imatinib-d8 normalized to a $400 \mathrm{mg}$ imatinib dose was $60.5 \pm 26.4 \mu \mathrm{g} \cdot \mathrm{h} / \mathrm{mL}$. Individual plasma concentration-time curves demonstrated up to two secondary peaks after the $C_{\max }$, with different profiles for oral imatinib and intravenous imatinib-d8 (Supplementary Figure 1). The ratios between the curves for oral imatinib and intravenous imatinib-d8 remained constant during the elimination phase, with a dose-normalized imatinib:imatinib-d 8 ratio in plasma of 2.00 at $t=6 \mathrm{~h}$ and of 2.04 at $t=24 \mathrm{~h}$. The $t_{1 / 2}$ and clearance of imatinib-d 8 were $45.5 \mathrm{~h}$ and $7.6 \mathrm{~L} / \mathrm{h}$, respectively.

The absolute bioavailability $(F)$ of oral imatinib at steady state was calculated for each individual subject. Table 3 demonstrates that the median absolute bioavailability of oral imatinib in cancer patients was 76\% (range 42-106\%).

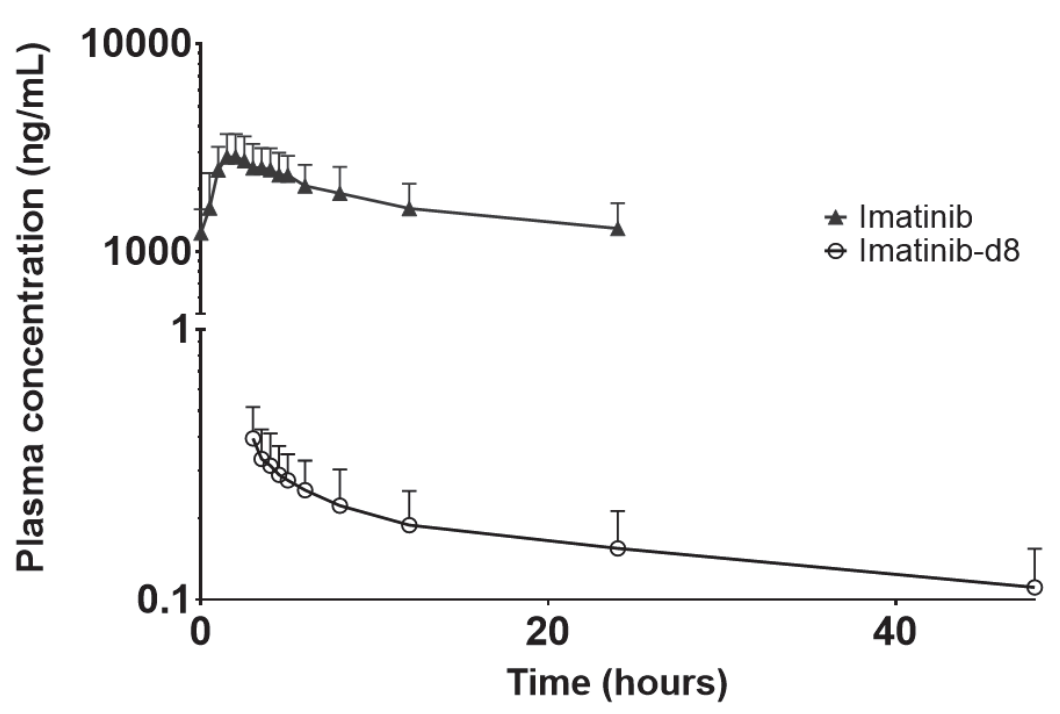

Figure 3 - Plasma concentration-time curves of imatinib and imatinib-d8 (mean \pm SD) following oral administration of $400 \mathrm{mg}$ imatinib dose at $\mathrm{t}=\mathbf{0 h}$ and intravenous administration of a $100 \mu \mathrm{g}$ imatinib- $\mathrm{d} 8$ microdose at $t=2.5 \mathrm{~h}$ in patients $(n=6)$ displaying steady state imatinib plasma pharmacokinetics

Table 2 - Summary of imatinib and imatinib-d8 steady state pharmacokinetic parameters following concomitant administration of an oral imatinib dose $(400 \mathrm{mg})$ and an intravenous imatinib-d8 microdose $(100 \mu \mathrm{g})$ in cancer patients $(n=6)$

\begin{tabular}{llll}
\hline Parameter & & Imatinib & Imatinib-d8 \\
\hline $\mathbf{C}_{\max }(\mu \mathrm{g} / \mathrm{mL})$ & Mean & 2.9 & 0.00051 \\
\cline { 2 - 4 } & $\mathrm{CV}(\%)$ & 27.4 & 23.1 \\
\hline $\mathbf{C}_{\min , \mathbf{0 h}}(\mu \mathrm{g} / \mathrm{mL})$ & Mean & 1.2 & N/A \\
\cline { 2 - 4 } & $\mathrm{CV}(\%)$ & 27.4 & $\mathrm{~N} / \mathrm{A}$ \\
\hline $\mathbf{t}_{\max }(\mathrm{h})$ & Median & 2 & $\mathrm{~N} / \mathrm{A}$ \\
\cline { 2 - 4 } & Range & $1.5-2$ & $\mathrm{~N} / \mathrm{A}$ \\
\hline AUC $_{\mathbf{0 - 2 4}}(\mu \mathrm{g} \cdot \mathrm{h} / \mathrm{mL})$ & Mean & 42.6 & $\mathrm{~N} / \mathrm{A}$ \\
\cline { 2 - 4 } & $\mathrm{CV}(\%)$ & 30.2 & $\mathrm{~N} / \mathrm{A}$ \\
\hline
\end{tabular}




\begin{tabular}{|c|c|c|c|}
\hline \multirow[t]{2}{*}{ AUC $_{0 \text {-inf }}(\mu \mathrm{g} \cdot \mathrm{h} / \mathrm{mL})$} & Mean & N/A & 0.015 \\
\hline & CV (\%) & $\mathrm{N} / \mathrm{A}$ & 43.7 \\
\hline \multirow[t]{2}{*}{$\mathbf{t}_{1 / 2}(\mathrm{~h})$} & Mean & 34.1 & 45.5 \\
\hline & CV (\%) & 46.7 & 37.9 \\
\hline \multirow[t]{2}{*}{$\boldsymbol{k}_{\mathrm{e}}\left(\mathrm{h}^{-1}\right)$} & Mean & 0.023 & 0.017 \\
\hline & CV (\%) & 27.5 & 28.1 \\
\hline \multirow[t]{2}{*}{$\mathbf{V}_{\mathrm{d}} / \mathrm{F}(\mathrm{L})$} & Mean & 190 & N/A \\
\hline & CV (\%) & 26.7 & N/A \\
\hline \multirow[t]{2}{*}{$\mathbf{V}_{\mathbf{d}}(\mathrm{L})$} & Mean & N/A & 462 \\
\hline & CV (\%) & $\mathrm{N} / \mathrm{A}$ & 28.2 \\
\hline \multirow[t]{2}{*}{ CL/F (L/h) } & Mean & 4.2 & N/A \\
\hline & CV (\%) & 31.3 & N/A \\
\hline \multirow[t]{2}{*}{ CL (L/h) } & Mean & N/A & 7.6 \\
\hline & CV (\%) & $\mathrm{N} / \mathrm{A}$ & 36.1 \\
\hline
\end{tabular}

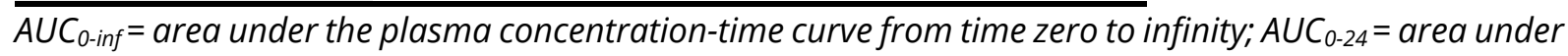
the plasma concentration-time curve from time zero to $24 \mathrm{~h} ; C L / F=$ apparent oral clearance; $C L=$ apparent total body clearance; $C_{\max }=$ maximum observed plasma concentration; $C_{\min }=$ minimum observed plasma concentration at $t=0 \mathrm{~h} ; \mathrm{CV}=$ coefficient of variation; $N / A=$ not applicable; $t_{\max }=$ time to reach maximum observed plasma concentration; $t_{1 / 2}=$ terminal half-life; $V_{d} / F=$ apparent volume of distribution after oral administration; $V_{d}=$ apparent volume of distribution.

Table 3 - Absolute bioavailability of oral imatinib at steady state plasma pharmacokinetics $(n=6)$

\begin{tabular}{lll}
\hline & $\begin{array}{l}\text { Imatinib } \\
\text { tablet } \\
(\mathbf{4 0 0} \mathbf{~ m g})\end{array}$ & $\begin{array}{l}\text { Intravenous imatinib- } \\
\mathbf{d} 8 \\
(\mathbf{1 0 0} \mu \mathrm{g})\end{array}$ \\
\hline AUC $_{\mathbf{0}-\mathbf{2 4}}(\mu \mathrm{g} \cdot \mathrm{h} / \mathrm{mL})(\mathrm{CV} \%)$ & $42.6(30.2)$ & $\mathrm{N} / \mathrm{A}$ \\
\hline AUC $_{\mathbf{0} \text {-inf }}(\mu \mathrm{g} \cdot \mathrm{h} / \mathrm{mL})(\mathrm{CV} \%)$ & $\mathrm{N} / \mathrm{A}$ & $0.015(43.7)$ \\
\hline $\begin{array}{l}\text { Dose normalized } \mathbf{A U C} \mathbf{0}_{\text {0-inf }}(\mu \mathrm{g} \cdot \mathrm{h} / \mathrm{mL}) \\
(\mathrm{CV} \%)\end{array}$ & $\mathrm{N} / \mathrm{A}$ & $60.5(43.7)$ \\
\hline $\mathbf{F}(\%)($ median, range) & & \\
\hline
\end{tabular}

\section{DISCUSSION}

The current study describes results on the determination of the absolute bioavailability of oral imatinib following concomitant administration of a single intravenous stable isotopically labeled imatinib-d8 microdose.

Technically, the stable isotopically microdose trial design proved successful. For all patients, imatinib and imatinib-d8 concentrations could be simultaneously quantified in all collected plasma samples. The quantification of imatinib-d8 was not biased by high concentrations of unlabeled imatinib present in the same plasma sample. In theory, the use of deuterium as a label for the intravenous microdose may result in a kinetic isotope effect (KIE), caused by increased bond strength of the carbon-deuterium bond, as 
compared with the carbon-hydrogen bond. The KIE may result in altered pharmacokinetics (e.g. altered metabolism) of the deuterium labeled drug, with an incorrect calculation of the absolute bioavailability as a result. ${ }^{6}$ As the deuterium labels in the imatinib-d8 structure were not located at metabolic hot spots in the imatinib molecule ${ }^{17}$, the KIE was assumed to be negligible. As seen in Figure 3, the curves for oral and intravenous imatinib demonstrate a parallel decline during the terminal elimination phase, with a constant mean dose-normalized imatinib:imatinib-d8 ratio in plasma of around 2.00, confirming that the KIE for the imatinib-d8 molecule was indeed negligible. The curves presented here demonstrate the validity of using the deuterium labeled imatinib-d8 drug molecule for intravenous microdose administration.

The median absolute bioavailability was calculated to be $76 \%$ which was less than the $98 \%$ (87-111\% (90\% confidence interval)) reported using a traditional two-period crossover design in healthy volunteers. ${ }^{18}$ There might be different reasons for the lower absolute bioavailability found in this study as compared with the study in healthy volunteers. In the previous absolute bioavailability trial, healthy volunteers demonstrated considerable inter-subject variation in the absolute bioavailability of imatinib in twelve treated subjects. ${ }^{1}$ The reasons for the high variability may be attributed to inter-subject variations in the activity of cytochrome P450 isoenzyme 3A4 (CYP3A4), a major enzyme in the biotransformation of imatinib. ${ }^{1}$ It could be that the lower bioavailability found in our study may solely be a result of this interpatient variability, as both studies demonstrate a relatively large inter-subject variability in small study populations (6 and 12 subjects included for each trial, respectively). An alternative theory may be that the absolute bioavailability actually differs between healthy volunteers and GIST patients. If so, there might be a change present at baseline, or a change developed during prolonged treatment with imatinib. In theory, GIST disease status may negatively influence the absorption of drug into the systemic circulation, resulting in a lower absolute bioavailability at baseline. In a previous study, patients with a prior major gastrectomy had a significantly lower $C_{\text {min, }}$ while other types of surgery were not associated with decreased pharmacokinetic exposure. ${ }^{19}$ However, in another observational study, type of surgery and extent of resection were not predictive of low imatinib concentrations. ${ }^{21}$ Our study patient population consisted of patients without prior major gastrectomy (Table 1), and results were therefore not likely to be influenced by prior surgery.

Another explanation for the lower bioavailability might be a change developed during prolonged imatinib treatment. Imatinib pharmacokinetic parameters have been described to change from early to later treatment phase, with a trend towards increased imatinib clearance after long-term exposure ${ }^{21,22}$, although this finding could not be reproduced in other studies. ${ }^{20,23}$ In our study population, all patients were on imatinib treatment for several months or years (median 3.2 years, range 0.3-13.0 years), and the clearance was similar to the clearance observed during the first month of treatment as 
described by Judson et al. (7.6 L/h vs. $9.2 \mathrm{~L} / \mathrm{h}) .{ }^{21}$ Since pharmacokinetic exposure to imatinib has been related to treatment efficacy ${ }^{24}$, therapeutic drug monitoring has been implemented in our hospital. Therefore, in case of an increased clearance and thus a lower pharmacokinetic exposure, dose escalations have probably been performed. These patients were not eligible for inclusion in this trial, which might explain the absence of an observed increase in drug clearance as a result of selection bias. Furthermore, if a change in clearance was found, this would not have explained the lower value for absolute bioavailability, as the oral and intravenous dose are co-administered during a single-dose event, eliminating inter-dose variability.

In a prospective pharmacokinetic trial on imatinib plasma concentrations in GIST patients, a reduced exposure of approximately $30 \%$ to imatinib was observed after long-term treatment (> 90 days), most likely due to reduced drug absorption over time. ${ }^{25}$ This reduced exposure may potentially be a result of the lower absolute bioavailability that we observed in our trial. Although different theories for this reduced absorption and/or bioavailability do exist (e.g. changed activity or expression of drug transporters involved in active transport, upregulation of (YP3A4 $)^{25}$, none have been confirmed to date.

Finally, the lower bioavailability found in our study could potentially be explained by the fact that patients ingested imatinib concomitant with food (according to the label), while the previous absolute bioavailability study has been performed under fasted conditions. Although a previous food-effect study concluded that food did not affect imatinib pharmacokinetics to a clinically relevant extent, $C_{\max }$ and $A \cup C_{0-24 h}$ decreased $15 \%$ and $9 \%$, respectively, after concomitant intake with a high-fat meal compared to the fasted state. ${ }^{26}$

Interestingly, the individual plasma concentration-time curves demonstrated up to two secondary peaks after the $C_{\max }$, with different profiles for oral imatinib and intravenous imatinib-d8 (Supplementary Figure 1). Previous research on imatinib has not demonstrated enterohepatic cycling of imatinib. Another explanation for these peaks might be bile secretion triggered by food intake, resulting in acceleration of drug solubility in the gastrointestinal lumen, although food has been described to have no relevant impact on the rate or extent of bioavailability. ${ }^{27}$

By using the stable isotopically labeled microdose trial design, the number of dose events and collected plasma samples were reduced by half, as compared with the previously performed absolute bioavailability trial using a conventional crossover design. ${ }^{18}$ This reduction may aid to perform this trial in patients in the future, as it offers the possibility to be combined with a phase I/II trial in patients without adding a separate intravenous dose event. The microdose trial design using a stable isotopically labeled drug will only mildly increase patient burden by adding a single intravenous microdose administration to the study procedures. This minor adjustment may result in increased and more 
relevant knowledge on the pharmacokinetics of a novel drug product in an early stage of clinical drug development.

\section{CONCLUSION}

The absolute bioavailability of oral imatinib in cancer patients during steady state pharmacokinetics was successfully determined using a stable isotopically labeled microdose trial. This study demonstrates the potential to use a stable isotopically labeled microdose in combination with LC-MS/MS for the assessment of absolute bioavailability. In addition, the potential added value of performing an absolute bioavailability study in the intended patient population for clinical use during steady state pharmacokinetics was demonstrated by comparing the results obtained with a previously performed absolute bioavailability trial in healthy volunteers. 


\section{REFERENCES}

1. Liu G, Franssen E, Fitch MI, Warner E. Patient preferences for oral versus intravenous palliative chemotherapy.J Clin Oncol 1997; 15: 110-115.

2. Benjamin L, Cotté FE, Philippe C, Mercier F, Bachelot T, Vidal-Trécan G. Physicians' preferences for prescribing oral and intravenous anticancer drugs: A Discrete Choice Experiment. Eur J Cancer 2012; 48: 912-920.

3. Herbrink M, Nuijen B, Schellens JHM, Beijnen JH. Variability in bioavailability of small molecular tyrosine kinase inhibitors. Cancer Treat Rev 2015; 41: 412-422.

4. European Medicines Agency. Clinical pharmacology and pharmacokinetics: questions and answers. Available from: https://www.ema.europa.eu/en/human-regulatory/researchdevelopment/scientific-guidelines/clinical-pharmacology-pharmacokinetics/clinicalpharmacology-pharmacokinetics-questions-answers.

5. U.S. Food and Drug Administration. Bioavailability Studies Submitted in NDAs or INDs General Considerations Guidance for Industry. 2019. Available from: https://www.fda.gov/media/121311/download

6. Lappin G, Rowland M, Garner RC. The use of isotopes in the determination of absolute bioavailability of drugs in humans. Expert Opin Drug Metab Toxicol 2006; 2: 419-427.

7. European Medicines Agency. ICH M3(R2)-guidance on non-clinical safety studies for the conduct of human clinical trials and marketing authorization for pharmaceuticals. Available from: https://database.ich.org/sites/default/files/M3_R2_Guideline.pdf.

8. $\mathrm{Xu} \mathrm{X}$, Jiang $\mathrm{H}$, Christopher LJ, Shen JX, Zeng J, Arnold ME. Sensitivity-based analytical approaches to support human absolute bioavailability studies. Bioanalysis 2014; 6: 497-504.

9. Burt T, John CS, Ruckle JL, Vuong LT. Phase-0/microdosing studies using PET, AMS, and LCMS/MS: a range of study methodologies and conduct considerations. Accelerating development of novel pharmaceuticals through safe testing in humans-a practical guide. Expert Opin Drug Deliv 2017; 14: 657-672.

10. Lappin G. Approaches to intravenous clinical pharmacokinetics: recent developments with isotopic microtracers. J Clin Pharmacol 2016; 56: 11-23.

11. European Medicines Agency. Glivec - Summary of Product Characteristics. Available from: https://www.ema.europa.eu/en/documents/product-information/glivec-epar-productinformation_en.pdf.

12. Roosendaal J, Venekamp N, Lucas L, Rosing H, Beijnen JH. Development and validation of an LC-MS/MS method for the quantification of imatinib and imatinib-d8 in human plasma for the support of an absolute bioavailability microdose trial. Pharmazie 2020; 75: 136-141.

13. U.S. Food and Drug Administration. Guidance for industry: bioanalytical method validation. 2018. Available from: https://www.fda.gov/media/70858/download.

14. European Medicines Agency. Guideline on bioanalytical method validation. 2011. Available from: https://www.ema.europa.eu/en/documents/scientific-guideline/guidelinebioanalytical-method-validation_en.pdf.

15. R Development Core Team. R Core Team. R: a language and environment for statistical computing. 2008. Available from: http://www.r-project.org. 
16. Rowland M, Tozer TN. Clinical pharmacokinetics - concepts and applications. 3rd ed. 1995.

17. Gschwind HP, Pfaar U, Waldmeier F, Zollinger M, Sayer C, Gross G. Metabolism and disposition of imatinib mesylate in healthy volunteers. Drug Metab Dispos 2005; 33: 15031512.

18. Peng B, Dutreix C, Mehring G, Hayes MJ, Ben-Am M, Seiberling $M$ et al. Absolute bioavailability of imatinib (Glivec ${ }^{\circledR}$ ) orally versus intravenous infusion.J Clin Pharmacol 2004; 44: 158-162.

19. Yoo C, Ryu MH, Kang BW, Yoon SK, Ryoo BY, Chang HM et al. Cross-sectional study of imatinib plasma trough levels in patients with advanced gastrointestinal stromal tumors: impact of gastrointestinal resection on exposure to imatinib. J Clin Oncol 2010; 28: 15541559.

20. Farag S, Verheijen RB, Martijn Kerst J, Cats A, Huitema ADR, Steeghs N. Imatinib pharmacokinetics in a large observational cohort of gastrointestinal stromal tumour patients. Clin Pharmacokinet 2017; 56: 287-292.

21. Judson I, Ma P, Peng B, Verweij J, Racine A, Di Paola ED et al. Imatinib pharmacokinetics in patients with gastrointestinal stromal tumour: a retrospective population pharmacokinetic study over time. EORTC Soft Tissue and Bone Sarcoma Group. Cancer Chemother Pharmacol 2005; 55: 379-386.

22. Bins S, Eechoute K, Kloth JSL, de Man FM, Oosten AW, de Bruijn P et al. Prospective analysis in GIST patients on the role of alpha-1 acid glycoprotein in imatinib exposure. Clin Pharmacokinet 2017; 56: 305-310.

23. Yoo C, Ryu MH, Ryoo BY, Beck MY, Chang HM, Lee JL et al. Changes in imatinib plasma trough level during long-term treatment of patients with advanced gastrointestinal stromal tumors: correlation between changes in covariates and imatinib exposure. Invest New Drugs 2012; 30: 1703-1708.

24. Demetri GD, Wang Y, Wehrle E, Racine A, Nikolova Z, Blanke CD et al. Imatinib plasma levels are correlated with clinical benefit in patients with unresectable/metastatic gastrointestinal stromal tumors. J Clin Oncol 2009; 27: 3141-3147.

25. Eechoute K, Fransson MN, Reyners AK, De Jong FA, Sparreboom A, Van Der Graaf WTA et al. A long-term prospective population pharmacokinetic study on imatinib plasma concentrations in GIST patients. Clin Cancer Res 2012; 18: 5780-5787.

26. U.S. Food and Drug Administration. Gleevec Clinical Pharmacology and Biopharmaceutics Review. $2001 . \quad$ Available from: https://www.accessdata.fda.gov/drugsatfda_docs/nda/2001/21335_Gleevec_biopharmr_P1.pdf.

27. Peng B, Lloyd P, Schran H. Clinical pharmacokinetics of imatinib. Clin Pharmacokinet 2005; 44: 879-894. 


\section{SUPPLEMENTARY DATA}

\section{Imatinib (p.o.)}

A

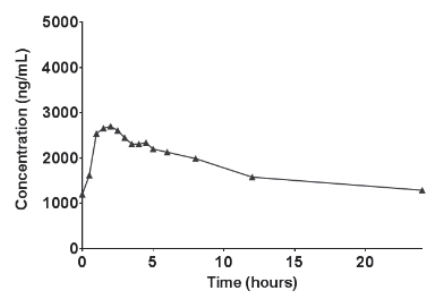

B

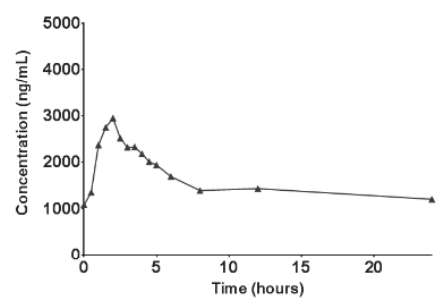

C

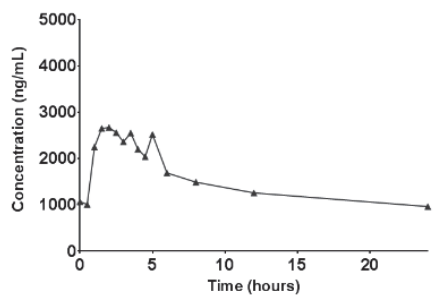

$\mathbf{D}$

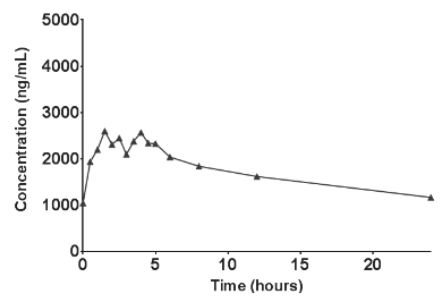

$\mathbf{E}$

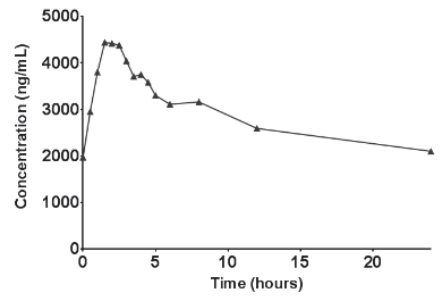

$\mathbf{F}$

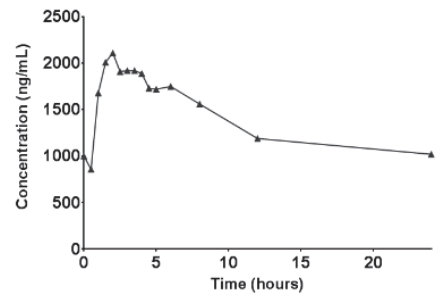

Imatinib-d8 (i.v.)
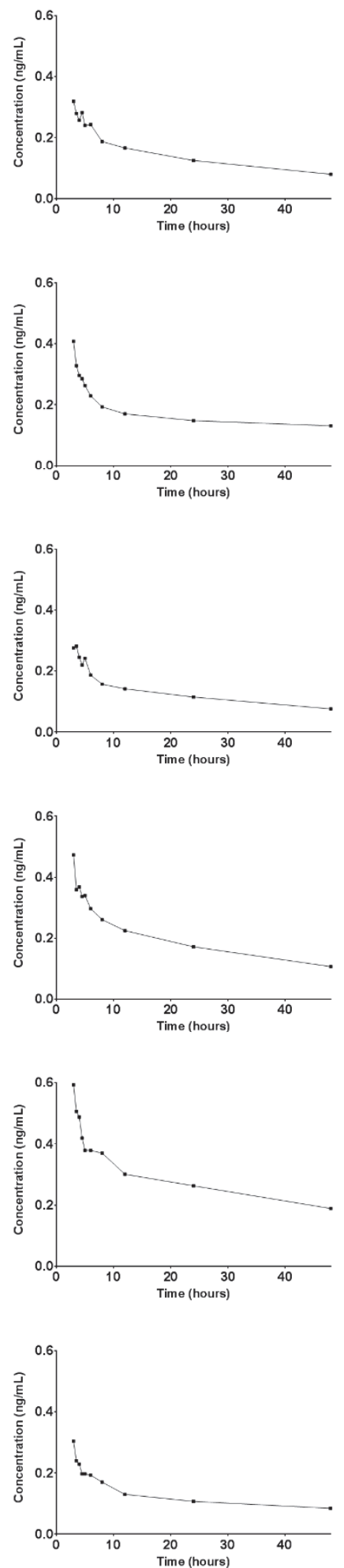

Supplementary Figure 1 - Plasma concentration-time curves of imatinib and imatinib-d8 following oral (p.o.) administration of $400 \mathrm{mg}$ imatinib dose at $t=0 \mathrm{~h}$ and intravenous (i.v.) administration of a $100 \mu \mathrm{g}$ imatinib-d8 microdose at $t=2.5 \mathrm{~h}$ in patients $(1-6, \mathrm{~A}-\mathrm{F})$ displaying steady state imatinib plasma pharmacokinetics 



\section{Conclusions and perspectives}




\section{CONCLUSIONS AND PERSPECTIVES}

This thesis focuses on precision dosing of oral targeted therapies in oncology. Key points extracted from this thesis and the lessons learned are summarized in Figure $\mathbf{1}$ and discussed in more detail in the following paragraphs.

In short, the path forward for precision dosing in oncology can be visualized as a doubleedged sword, but with both sides of the blade contributing towards more rational precision medicine by optimizing exposure.

On the one side, we should gain a better understanding of the sources of variability in exposure. These factors should be controlled where possible in order to minimize the variability within and between patients. Selection of the right starting dose for each individual (group of) patient(s) will result in a decreased proportion of patients that are treated outside the therapeutic window in the first place, i.e. "right-dose-first-time" paradigm.

On the other side, we should accept that a certain degree of unexplained variability will always remain. Therefore, we should work around it by developing dose-adaptation strategies that result in exposures matching the target. In this way, we can titrate individual patients towards the therapeutic window by PK-guided dosing.

This paradigm is illustrated in the Graphical summary of this thesis, in which it is indicated how each chapter connects to it. 


\section{LESSONS (TO BE) LEARNED}

\section{Understanding and minimizing variability}

Oral targeted therapies exhibit a large interindividual variability in pharmacokinetic exposure, caused by many factors. We should gain a better understanding of the sources of variability and minimize them where possible. In this way, fewer patients will be dosed outside the therapeutic window, i.e. "right-dose-first-time" paradigm.

\section{Pharmacokinetic exposure is related to response}

Pharmacokinetic exposure to many oral targeted therapies is related to both efficacy and toxicity. At the currently used fixed doses, a substantial subset of patients is under- or overexposed, providing a strong rationale for precision dosing.

\section{Precision dosing is ready for prime time}

PK-guided dosing was demonstrated to be feasible for many oral targeted therapies and resulted in more patients reaching the target exposure. Therefore, the stage is set for the implementation of PKguided dosing in routine clinical practice. Cost-neutral interventions can be applied if possible.

\section{Real-life data collection}

Preferably, both exposure-response analyses and feasibility studies are performed in a real-life setting, rather than in highly selected patients included in clinical trials. After the implementation of precision dosing, the collection of real-life data in registries should continue to further optimize the precision dosing approach.

\section{Precision dosing should be part of drug development}

Phase I/II studies should aim to define a target exposure instead of a (maximum tolerated) fixed dose. The feasibility of precision dosing can then be investigated in phase II/III studies. In this way, precision dosing will be an integral part of drug development.

Figure 1 - Key points extracted from this thesis and the lessons (to be) learned $P K=$ pharmacokinetically 


\section{UNDERSTANDING AND MINIMIZING VARIABILITY}

First of all, this thesis underlines that the variability in pharmacokinetic exposure between patients treated at the same dose is high for the oral targeted therapies that were studied, with coefficients of variation in minimum plasma concentration $\left(C_{\mathrm{min}}\right)$ of around $40-70 \%$ (Chapter 3, 4, 7, 10, 11, 14). As illustrated in the hallmarks of variability in the Graphical summary, many factors contribute to this high inter- and intra-individual variability. Several of these sources of variability have been addressed in the work described in this thesis.

It starts with a proper pharmaceutical formulation, as a higher bioavailability results in a reduced inter- and intra-individual variability in pharmacokinetic exposure. ${ }^{1,2}$ Currently, the absolute bioavailability is not determined for over half of the oral targeted therapies. ${ }^{3}$ The innovative clinical trial design used in Chapter $\mathbf{1 6}$ provides a more efficient way to conduct absolute bioavailability trials. ${ }^{4}$ Notably, the low bioavailability of pazopanib (i.e. 14-39\%) is a result of its poor solubility and its relative bioavailability was predicted to be higher at lower doses. ${ }^{5,6}$ Therefore, splitting intake moments can be used to increase the relative bioavailability and thereby boost pazopanib exposure (Chapter 12 and 13).

Other hallmarks of variability are drug-food and drug-drug interactions. ${ }^{7,8}$ Pharmacokinetic exposure of many oral targeted therapies is dramatically affected by gastric acid-suppressive agents (as we show for three patients in Chapter 13) and cytochrome P 450 3A4 (CYP3A4) modulators (as we summarize for cyclin-dependent kinase 4 and 6 (CDK4/6) inhibitors in Chapter 14). It is important to quantify the effect sizes of these drug-drug interactions to inform on appropriate dose adjustments in routine clinical management, as we did in Chapter 15. As described in Chapter 11 and 13, the food-effect can sometimes be leveraged to optimize exposure in patients who are underdosed.

Moreover, (patho-)physiological differences between patients can affect drug absorption, distribution, metabolism and excretion. Another key determinant in the variability in pharmacokinetic exposure is treatment adherence. ${ }^{9}$ In the prospective study on PKguided dosing described in Chapter 6, 7, 11 and 13, compliance was first evaluated with the patient in case of low exposure, before any PK-guided interventions were implemented. Lastly, pharmacogenomics can explain part of the observed variability in pharmacokinetic exposure. As many oral targeted therapies are extensively metabolized by cytochrome $\mathrm{P} 450$ enzymes, polymorphisms in these enzymes, can result in an altered clearance. ${ }^{10,11}$

Taken together, it is essential to enhance our knowledge on how each of these factors contributes to the variability in pharmacokinetic exposure within and between patients. In this way, variability can be reduced and the fraction of patients that are treated outside 
the therapeutic window can thereby be reduced. Nevertheless, unexplained variability will always remain, providing a strong rationale for PK-guided dosing (Chapter 1). In fact, measured drug concentrations are the translation of all known and unknown factors influencing variability.

\section{PHARMACOKINETIC EXPOSURE IS RELATED TO RESPONSE}

The presence of defined and consistent exposure-response relationships is the main reason to apply precision dosing, as described in Chapter 1. Pharmacokinetic exposure to many oral targeted therapies is related to both efficacy and toxicity. ${ }^{12,13}$ In Chapter 3-5 of this thesis, this is demonstrated for several of these compounds in real-life patient cohorts (i.e. abiraterone, crizotinib, alectinib and trametinib). In all three studies, a substantial subset of patients was underexposed (i.e. 37-63\%) at the currently used fixed dosing regimens.

However, not for all oral targeted therapies clinically relevant exposure-response relationships were identified. For example, in Chapter 5, pharmacokinetic exposure to dabrafenib could not be related to either efficacy or toxicity. Also, previous studies did not demonstrate an exposure-response relationship for enzalutamide and osimertinib. ${ }^{14,15}$ This may be explained by a plateau in the exposure-response curve for these drugs, that are dosed at the flat end of this curve. For some molecular targets (i.e. BRAF and EGFR) the therapeutic window appears to be wider than for others (i.e. ALK, MEK and VEGF). Also, newer generation kinase inhibitors may have a more robust formulation resulting in reduced variability.

It is thus essential to study for which oral targeted therapies precision dosing holds promise, and for which it might not be worthwhile. Before solid conclusions can be drawn on the absence of an exposure-response relationship, it should be ensured that the study has sufficient power to demonstrate this. Otherwise, absence of evidence is not evidence of absence, as is the case for several underpowered exposure-response analyses for endoxifen. ${ }^{16-18}$

Although dose reductions do not need to be actively recommended in case of high exposure and manageable tolerability, measuring drug concentrations can support dose reductions in case of toxicity. In Chapter 9, two patients are described who maintained adequate exposure at an eight times lower than standard dose of pazopanib. According to the drug label, this dose was considered insufficient to obtain a clinically relevant effect, and without PK-guided dosing, treatment would definitely have been discontinued in these patients. Adequate drug levels can thus support to continue treatment at a reduced dose, while low drug levels may justify switching therapy. 


\section{PRECISION DOSING IS READY FOR PRIME TIME}

In this thesis, it is argued why the fixed dosing paradigm should be left (Chapter $\mathbf{1}$ and 2), and the stage is set for the implementation of precision dosing. For drugs with clearly proven exposure-efficacy relationships (Chapter 3-5 and 10), for which PK-guided dose adaptation was demonstrated to be feasible and resulted in more patients reaching the target exposure (Chapter 7, 8, 11 and 13), PK-guided dosing should be implemented as part of routine clinical management.

Notably, PK-based dose adjustments are already routinely made for many drugs and are recommended by health authorities, e.g. for patients with renal impairment or for drugdrug interaction management. ${ }^{19-22}$ Precision dosing simply extrapolates this exposure matching paradigm from selected patient populations to each individual patient with a suboptimal exposure, irrespective of the underlying cause. If it has been demonstrated that exposure is related to a relevant clinical outcome (i.e. efficacy or toxicity) and that exposure can be improved by PK-guided dosing, it could be logically assumed that PKguided dosing would result in better treatment outcomes. Therefore, as argued in Chapter 2, confirmatory randomized trials are not needed to demonstrate the clinical relevance of precision dosing.

Furthermore, the right starting dose can be selected for each individual (group of) patients, e.g. based on pharmacogenomics or by appropriate management of drug-drug interactions (as shown in Chapter 15), i.e. "right-dose-first-time" paradigm, after which this dose can be further optimized by PK-guided dosing.

Our focus should now be twofold. On the one hand, we should continue to establish exposure-response relationships and perform feasibility studies for PK-guided dosing of new oral targeted therapies. On the other hand, we should ensure that precision dosing will be implemented in routine clinical practice. As described in Chapter 7, the threestage-design of the Dutch Pharmacology Oncology Group - Therapeutic Drug Monitoring (DPOG-TDM) study provides a framework to perform feasibility studies and subsequently enables further nationwide implementation using the existing infrastructure of the DPOGTDM study. This systematic approach can also be used internationally for this purpose, within the same protocol or by setting up similar frameworks in other countries.

Unlike certain other classes of drugs for which TDM is advocated, that are aimed at exogenous targets (e.g. anti-infectious drugs), oral targeted therapies are aimed at targets originating from endogenous proteins. The inherently small therapeutic indices associated with some of these targets imply that the need for precision dosing will remain in the foreseeable future, despite the development of newer generation inhibitors with optimized pharmaceutical formulations. This further stresses the importance of investing 
in a solid infrastructure for PK-guided dosing (i.e. sample collection, shipment and measurement, with a short turn-around time).

Increased treatment costs for higher than approved dosages will remain a concern. In Chapter 11-13 of this thesis, we provide examples of cost-neutral interventions that could be performed as first steps in case of low exposure. Moreover, absolute dose increments have been proven cost-effective for several anticancer drugs, including imatinib and tamoxifen. ${ }^{23-25}$ Ideally, though, prices would be determined per treatment, regardless of the administered dose.

\section{REAL-LIFE DATA COLLECTION}

Preferably, both exposure-response analyses and feasibility studies are performed in a real-life setting, as were the studies described in this thesis (Chapter 3-9, 11-13, 15 and 16), rather than in highly selected patients included in clinical trials. This will provide a more accurate reflection of clinical practice. Due to strict eligibility criteria, patients enrolled in clinical trials may differ from those in clinical practice, limiting the generalizability of these studies. ${ }^{26,27}$ Mainly, interindividual variability in PK exposure is higher in real-life patients, as this is a far more diverse population with more comorbidities and concomitant medication. Also, treatment adherence is strictly monitored in clinical trials, while this may be monitored less stringently in clinical practice. Furthermore, treatment outcomes are often better in patients treated in clinical trials (i.e. efficacy), compared with patients receiving the same treatment under real-world circumstances (i.e. effectiveness), which is referred to as the efficacy-effectiveness gap. ${ }^{28,29}$ Downsides of real-world studies, though, include the risk of confounding and the uncertainties about the quality of the collected data. These limitations should be adequately addressed to prevent that flawed conclusions are drawn, i.e. by taking known prognostic factors into account in multivariable analyses and by building registries to prospectively capture data.

Also after implementation of precision dosing in routine care, the collection of real-life data in registries should continue. In this way, the precision dosing strategy can be further improved with data of additional patients. The third-stage cohorts of the DPOG-TDM study can be used for this purpose. In addition, large international collaborations and pooled analyses will maximize what can be learned from these data. Especially in this setting, registries that are integrated with electronic patient files will enable timely and efficient data collection.

\section{PRECISION DOSING SHOULD BE PART OF DRUG DEVELOPMENT}

The work described in this thesis emphasizes the important role of the discipline of clinical pharmacology in (early) drug development. As proposed by Lewis Sheiner, cycles of 
learning and confirming should be alternated throughout a drug's life cycle. ${ }^{30}$ In fact, incentives by regulatory authorities have now resulted in a drug's pharmacokinetics and pharmacodynamics being more extensively studied. Yet, more action can be taken upon this knowledge to optimize treatment.

More attention should be paid to optimize pharmaceutical formulations, which will help to increase bioavailability and reduce variability. In addition, intra-individual variability needs to be characterized in more detail, as this increases our understanding of the pharmacokinetic behavior of a new drug. ${ }^{31}$ Furthermore, efforts should be made to shorten the delay between the approval of a new drug and the identification of a suitable PK target for precision dosing. To illustrate this delay, imatinib was approved for the treatment of chronic myeloid leukemia in 2001, while the first publication on its exposureresponse relationship appeared only in 2007.32 Similarly, pazopanib was marketed in 2010, whereas it took until 2014 before its efficacy threshold was proposed. ${ }^{33}$ A major stride can be made by defining a target exposure in phase I/II studies, instead of a (maximum tolerated) fixed dose for all patients, provided that enough data is available to allow for meaningful analyses. The feasibility of precision dosing can then be evaluated in phase II/III studies. In this way, precision dosing will be an integral part of drug development.

\section{CONCLUDING REMARKS}

In this thesis, the rationale (Part I), the evidence (Part II) and the clinical application (Part III and IV) of precision dosing of oral targeted therapies in oncology are described. Based on the high interindividual variability and exposure-response relationships, it is concluded that there is a strong rationale for precision dosing. Moreover, PK-guided dosing was feasible in clinical practice for several compounds and resulted in more patients reaching the target exposure. Taken together, precision dosing has the potential to further optimize treatment with oral targeted therapies. Therefore, efforts should be made to implement precision dosing in routine clinical practice. Furthermore, the path forward should focus on investigating exposure-response relationships of new oral targeted therapies, identify optimal thresholds for PK-guided dosing and study the feasibility of precision dosing for these new compounds. Ideally, target exposures should be defined in early phase clinical trials to make precision dosing an integral part of drug development. 


\section{REFERENCES}

1. Hellriegel ET, Bjornsson TD, Hauck WW. Interpatient variability in bioavailability is related to the extent of absorption: implications for bioavailability and bioequivalence studies. Clin Pharmacol Ther 1996; 60: 601-607.

2. Herbrink M, Nuijen B, Schellens JHM, Beijnen JH. High-Tech Drugs in Creaky Formulations. Pharm Res 2017; 34: 1751-1753.

3. Herbrink M, Nuijen B, Schellens JHM, Beijnen JH. Variability in bioavailability of small molecular tyrosine kinase inhibitors. Cancer Treat Rev 2015; 41: 412-422.

4. Roosendaal J, Rosing $\mathrm{H}$, Beijnen $\mathrm{JH}$. Stable isotopically labeled intravenous microdose pharmacokinetic trials as a tool to assess absolute bioavailability: feasibility and paradigm to apply for protein kinase inhibitors in oncology. Clin Pharmacol Drug Dev 2020; 9: 552-559.

5. Deng Y, Sychterz C, Suttle AB, Dar MM, Bershas D, Negash K et al. Bioavailability, metabolism and disposition of oral pazopanib in patients with advanced cancer. Xenobiotica 2013; 43: 443-453.

6. Yu H, van Erp N, Bins S, Mathijssen RHJ, Schellens JHM, Beijnen JH et al. Development of a pharmacokinetic model to describe the complex pharmacokinetics of pazopanib in cancer patients. Clin Pharmacokinet 2017; 56: 293-303.

7. Veerman GDM, Hussaarts KGAM, Jansman FGA, Koolen SWL, van Leeuwen RWF, Mathijssen $\mathrm{RHJ}$. Clinical implications of food-drug interactions with small-molecule kinase inhibitors. Lancet Oncol 2020; 21: e265-e279.

8. Hussaarts KGAM, Veerman GDM, Jansman FGA, van Gelder T, Mathijssen RHJ, van Leeuwen RWF. Clinically relevant drug interactions with multikinase inhibitors: a review. Ther Adv Med Oncol 2019; 11: 1-34.

9. Cardoso E, Csajka C, Schneider MP, Widmer N. Effect of adherence on pharmacokinetic/pharmacodynamic relationships of oral targeted anticancer drugs. Clin Pharmacokinet 2017; 57: 1-6.

10. Bins S, Huitema ADR, Laven P, Bouazzaoui S el, Yu H, van Erp N et al. Impact of CYP3A4*22 on pazopanib pharmacokinetics in cancer patients. Clin Pharmacokinet 2019; 58: 651-658.

11. Diekstra MHM, Klümpen HJ, Lolkema MPJK, Yu H, Kloth JSL, Gelderblom H et al. Association analysis of genetic polymorphisms in genes related to sunitinib pharmacokinetics, specifically clearance of sunitinib and SU12662. Clin Pharmacol Ther 2014; 96: 81-89.

12. Verheijen RB, Yu H, Schellens JHM, Beijnen JH, Steeghs N, Huitema ADR. Practical recommendations for therapeutic drug monitoring of kinase inhibitors in oncology. Clin Pharmacol Ther 2017; 102: 765-776.

13. Widmer N, Bardin C, Chatelut E, Paci A, Beijnen J, Levêque D et al. Review of therapeutic drug monitoring of anticancer drugs part two - targeted therapies. Eur J Cancer 2014; 50: 20202036.

14. van Nuland $M$, Bergman AM, Rosing $H$, de Vries $N$, Huitema ADR, Beijnen JH. Exposureresponse assessment of enzalutamide and its major metabolites in a real-world cohort of patients with metastatic castration-resistant prostate cancer. Pharmacotherapy 2019; 39: 
$1137-1145$

15. Brown K, Comisar C, Witjes H, Maringwa J, De Greef R, Vishwanathan K et al. Population pharmacokinetics and exposure-response of osimertinib in patients with non-small cell lung cancer. Br J Clin Pharmacol 2017; 83: 1216-1226.

16. Sanchez-Spitman A, Dezentje V, Swen J, Moes DJAR, Bohringer S, Batman E et al. Tamoxifen pharmacogenetics and metabolism: results from the prospective CYPTAM study.J Clin Oncol 2019; 37: 636-646.

17. Braal CL, Beijnen JH, Koolen SLW, Oomen-de Hoop E, Steeghs N, Jager A et al. Relevance of endoxifen concentrations: absence of evidence is not evidence of absence. J Clin Oncol 2019; 37: 1980-1981.

18. Sanchez-Spitman AB, Moes DJAR, Swen JJ, Dezentjé VO, Lambrechts $D$, Neven $P$ et al. Exposure-response analysis of endoxifen serum concentrations in early-breast cancer. Cancer Chemother Pharmacol 2020; 85: 1141-1152.

19. Food and Drug Administration. Center for Drug Evaluation and Research. Guidance for industry: pharmacokinetics in patients with impaired renal function - study design, data analysis, and impact on dosing and labeling. 2010. Available from: https://www.fda.gov/media/78573/download.

20. Committee for Medicinal Products for Human Use. European Medicines Agency. Guideline on the evaluation of the pharmacokinetics of medicinal products in patients with decreased renal function. 2016. Available from: https://www.ema.europa.eu/en/documents/scientificguideline/guideline-evaluation-pharmacokinetics-medicinal-products-patients-decreasedrenal-function_en.pdf.

21. Food and Drug Administration. Center for Drug Evaluation and Research. Clinical drug interaction studies - cytochrome P450 enzyme- and transportermediated drug interactions guidance for industry. 2020. Available from: https://www.fda.gov/media/134581/download.

22. Committee for Medicinal Products for Human Use. European Medicines Agency. Guideline on the investigation of drug interactions. 2012. Available from: http://www.ema.europa.eu/docs/en_GB/document_library/Scientific_guideline/2012 /07/WC500129606.pdf.

23. Zuidema S, Desar IME, van Erp NP, Kievit W. Optimizing the dose in patients treated with imatinib as first line treatment for gastrointestinal stromal tumours: a cost-effectiveness study. Br J Clin Pharmacol 2019; 85: 1994-2001.

24. van Nuland M, Vreman RA, ten Ham RMT, de Vries Schultink AHM, Rosing H, Schellens JHM et al. Cost-effectiveness of monitoring endoxifen levels in breast cancer patients adjuvantly treated with tamoxifen. Breast Cancer Res Treat 2018; 172: 143-150.

25. Vithanachchi DT, Maujean A, Downes MJ, Scuffham P. A comprehensive review of economic evaluations of therapeutic drug monitoring interventions for cancer treatments. $\mathrm{Br} \mathrm{J} C l i n$ Pharmacol. Online ahead of print 21 Jul 2020.

26. Sherman RE, Anderson SA, Dal Pan GJ, Gray GW, Gross T, Hunter NL et al. Real-world evidence - what is it and what can it tell us? N EnglJ Med 2016; 375: 2293-2297. 
27. Mitchell AP, Harrison MR, Walker MS, George DJ, Abernethy AP, Hirsch BR. Clinical trial participants with metastatic renal cell carcinoma differ from patients treated in real-world practice. J Oncol Pract 2015; 11: 491-497.

28. Templeton AJ, Booth CM, Tannock IF. Informing patients about expected outcomes: the efficacy-effectiveness gap. J Clin Oncol 2020; 38: 1651-1654.

29. Cramer-van der Welle CM, Peters BJM, Schramel FMNH, Klungel OH, Groen HJM, van de Garde EMW. Systematic evaluation of the efficacy-effectiveness gap of systemic treatments in metastatic nonsmall cell lung cancer. Eur Respir J 2018; 52: 1801100.

30. Sheiner LB. Learning versus confirming in clinical drug development. Clin Pharmacol Ther 1997; 61: 275-291.

31. Chatelut E, Bruno R, Ratain MJ. Intraindividual pharmacokinetic variability: focus on smallmolecule kinase inhibitors. Clin Pharmacol Ther 2018; 103: 956-958.

32. Picard S, Titier K, Etienne G, Teilhet E, Ducint D, Lassalle R et al. Trough imatinib plasma levels are associated with both cytogenetic and molecular responses to standard-dose imatinib in chronic myeloid leukemia. Blood 2007; 109: 3496-3499.

33. Suttle AB, Ball HA, Molimard M, Hutson T, Carpenter C, Rajagopalan D et al. Relationships between pazopanib exposure and clinical safety and efficacy in patients with advanced renal cell carcinoma. Br J Cancer 2014; 111: 1-8. 



\section{Graphical summary}

$D$

8

$\frac{1}{2}$

$\frac{1}{7}$

C

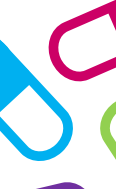

0

100

00

$0>$

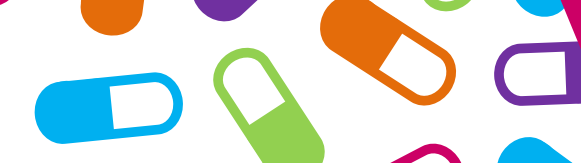

$0 \rightarrow 0$

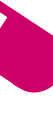

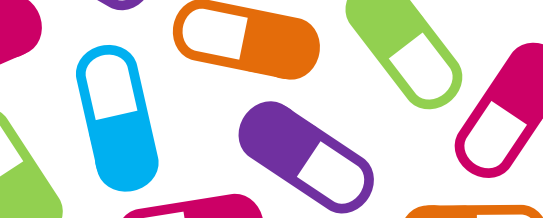




\section{UNDERSTANDING AND MINIMIZING VARIABILITY}

Gain a better understanding of the sources of variability in pharmacokinetic exposure.

Control these factors where possible, in order to minimize the variability and reduce

the proportion of patients treated outside the therapeutic window.

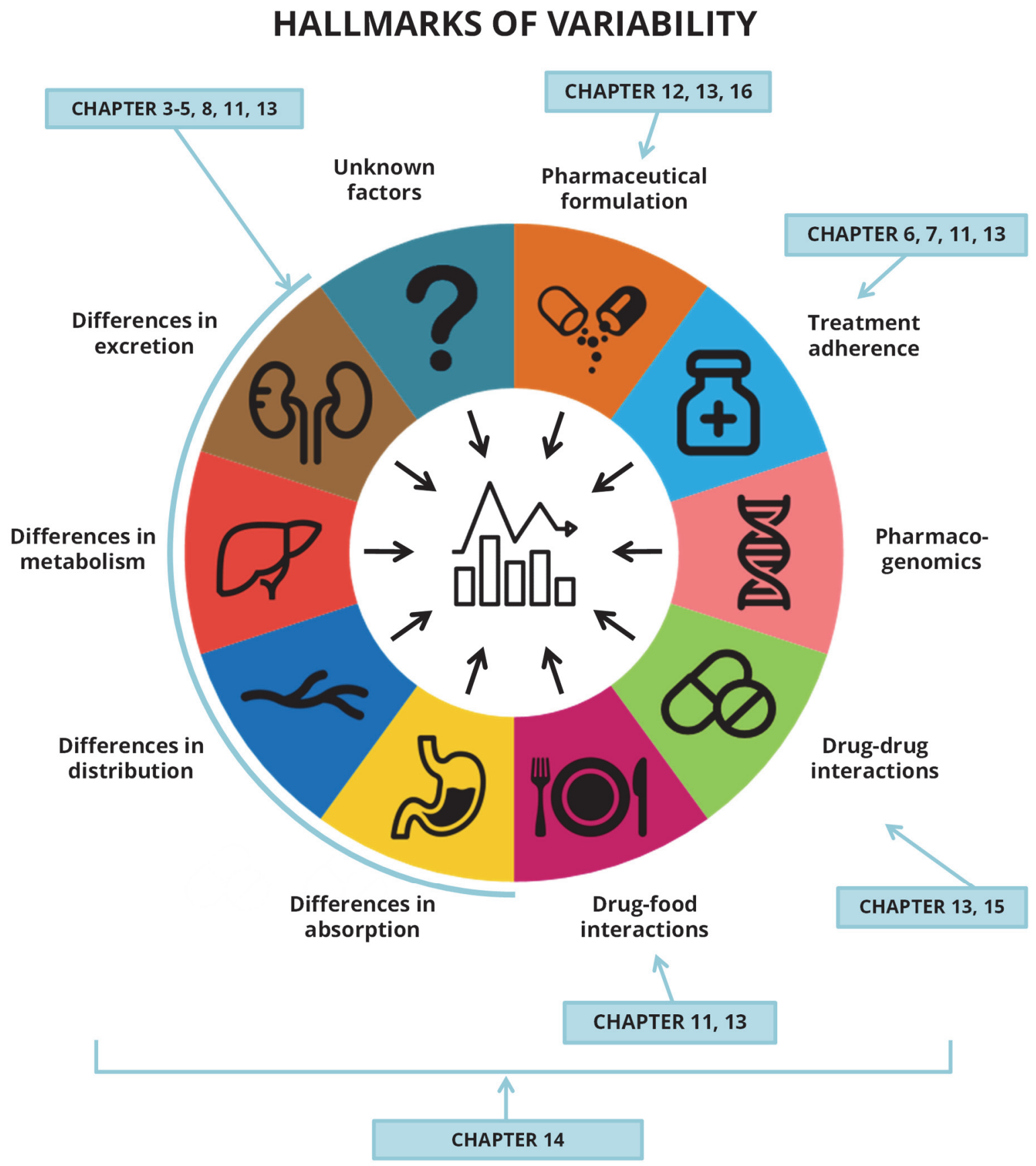

Graphical representation of the precision dosing paradigm described in this thesis.

The hallmarks of variability were designed in analogy to the hallmarks of cancer as described by Hanahan \& Weinberg. ${ }^{1}$ The height of the bars on the right side of the figure were chosen at random and do not represent a certain pharmacokinetic exposure or effect size of the PK-guided intervention. 


\section{PRECISION DOSING TO TITRATE INDIVIDUAL PATIENTS TOWARDS THE THERAPEUTIC WINDOW}

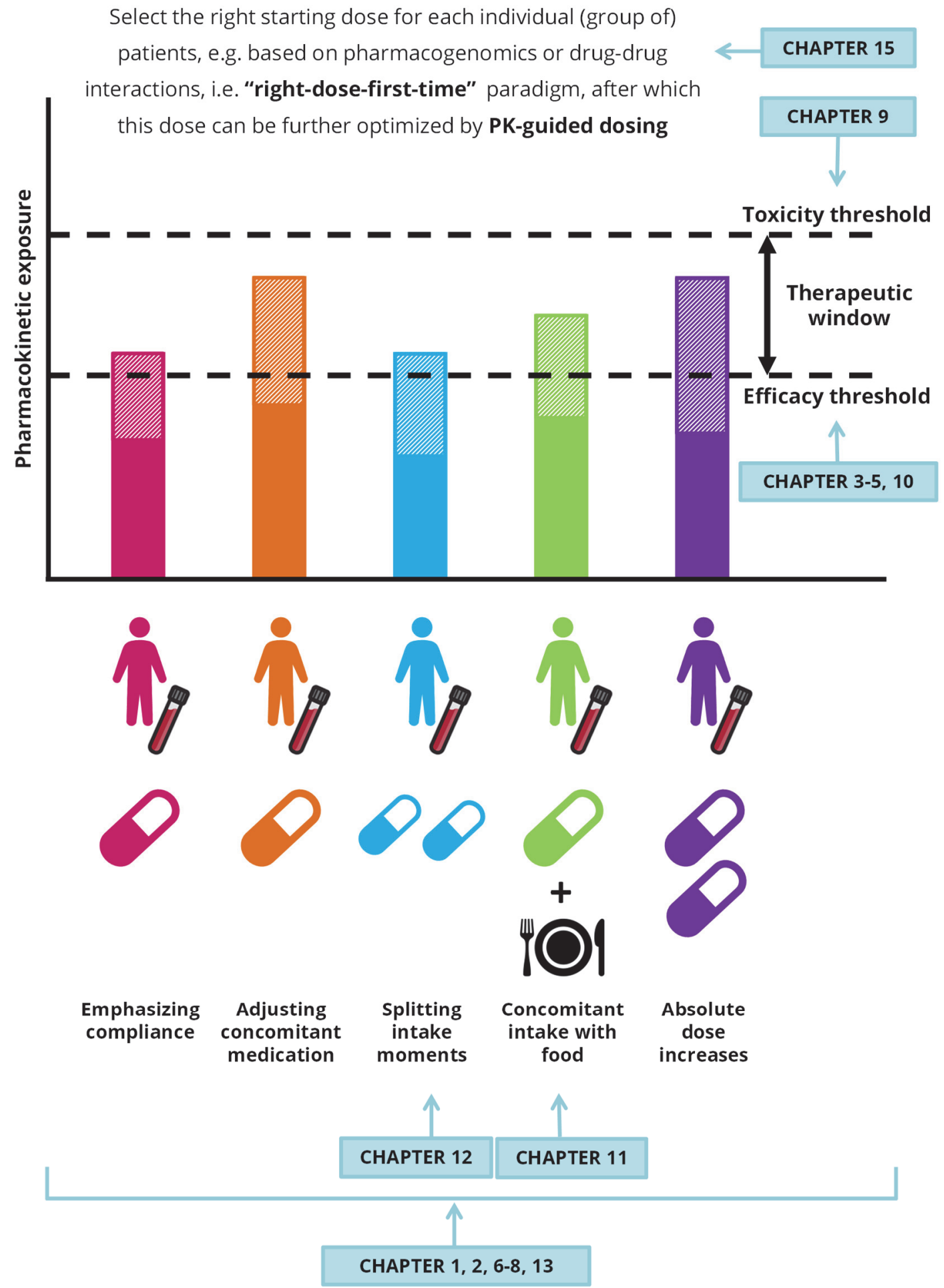




\section{REFERENCES}

1. Hanahan D, Weinberg RA. Hallmarks of cancer: the next generation. Cell 2011; 144: 646-674. 




\section{Summary}

$D$

0

$\frac{1}{I}$

$\frac{7}{7}$

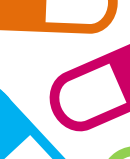

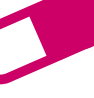

(

8

40

8

os

$\infty 80$

(

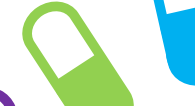

西

$\infty 8$

$\infty 8$

$\infty$ a

00000

g

P

$\checkmark a$

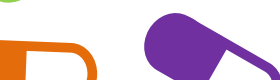

0 



\section{SUMMARY}

The advent of oral targeted therapies in oncology has widely been praised as a major breakthrough in the treatment of cancer. While our focus has increasingly shifted towards precision medicine, by selecting the right treatment based on molecular characteristics of the tumor, most of these novel anticancer drugs are still administered using a one-sizefits-all fixed dosing approach. However, pharmacokinetic (PK) characteristics of these new oral targeted therapies suggest precision dosing would be far more rational. Precision dosing can be achieved by selecting the right starting dose for each individual (group of) patient(s), i.e. "right-dose-first-time" paradigm, after which this dose can be further optimized by PK-guided dosing, i.e. adjusting the dose based on measured drug concentrations. This thesis focused on the rationale (Part I), the evidence (Part II) and the clinical application (Part III and IV) of precision dosing of oral targeted therapies in oncology.

\section{PART I - RATIONALE FOR PRECISION DOSING}

The aim of Chapter 1 was to address the scientific evidence for PK-guided dosing of oral targeted therapies by discussing the following criteria for its rational use:

1. absence of an easily measurable biomarker for drug effect;

2. long-term therapy;

3. availability of a validated sensitive bioanalytical method;

4. significant variability in pharmacokinetic exposure;

5. narrow therapeutic range;

6. defined and consistent exposure-response relationships;

7. feasible dose-adaptation strategies.

These requirements are met for most oral targeted anticancer drugs. Hence, to realize the full potential of precision medicine, we should not only treat patients with the right drug, but also at the right dose.

Although PK-guided dosing is rational and supported by an increasing body of evidence, it is still limitedly applied in oncology. An argument frequently used against its implementation in routine cancer care is the absence of randomized controlled trials (RCTs). In Chapter 2, it was argued why randomized confirmatory studies are not needed to demonstrate the clinical relevance of precision dosing. First, the inadequacy of the currently used fixed dosing paradigm and the progress made thus far in implementing precision dosing in oncology were discussed. Then, compelling arguments were laid out why large confirmatory RCTs are not necessary for the implementation of PK-guided dosing when clear exposure-efficacy relationships have been demonstrated. Finally, a path forward was proposed to demonstrate the clinical relevance of PK-guided dosing in oncology. 


\section{PART II - EXPOSURE-RESPONSE ANALYSES}

Before PK-guided dosing could be applied in clinical practice, sufficient evidence on exposure-efficacy relationships should be established and optimal thresholds for PKguided dosing need to be identified. For this purpose, it is essential that exposureresponse analyses are performed. In this part, exposure-response analyses were described for five oral targeted therapies: abiraterone, crizotinib, alectinib, dabrafenib and trametinib.

In Chapter 3, exposure-response analyses for abiraterone and its main metabolites were described. Abiraterone is an inhibitor of 17a-hydroxylase/C17,20-lyase (CYP17)and is used in the treatment of metastatic prostate cancer. Sixty-two patients were included in this observational study in which it was confirmed that the previously established threshold of abiraterone minimum plasma concentration $\left(C_{\min }\right)$ of $\geq 8.4 \mathrm{ng} / \mathrm{mL}$ is related to prolonged progression-free survival (PFS) in a real-life patient cohort (16.9 vs. 6.1 months, $\mathrm{p}=0.033$ ). Interindividual variability in exposure at the currently approved fixed dosing regimen was high, with a coefficient of variation of $70 \%$ for $C_{\min }$. As a result, a substantial subgroup of $42 \%$ of patients treated with abiraterone acetate is underexposed in routine care and at risk of suboptimal treatment efficacy.

Chapter 4 focused on anaplastic lymphoma kinase (ALK)-inhibitors crizotinib and alectinib, both indicated for the treatment of ALK-positive metastatic non-small-cell lung cancer. Exposure-response analyses were explored using previously proposed $C_{\text {min }}$ thresholds of $235 \mathrm{ng} / \mathrm{mL}$ for crizotinib and $435 \mathrm{ng} / \mathrm{mL}$ for alectinib. In 52 patients treated with alectinib, PFS was significantly longer in patients with a median $C_{\min } \geq 435 \mathrm{ng} / \mathrm{mL}$ compared to patients with an exposure below this threshold (not estimable (95\% confidence interval (Cl): 19.8-not estimable) vs. 12.6 months, $\mathrm{p}=0.04$ ). For crizotinib, a clinically relevant difference in median PFS was found as well, although this difference was only statistically significant in a pooled analysis including 79 patients with ALKpositive, ROS1-positive and cMET-dysregulated tumors (11.8 vs. 5.3 months, $p=0.04$ ). At the standard fixed dose, interindividual variability in exposure was high, with coefficients of variation for $\mathrm{C}_{\min }$ of $45 \%$ and $57 \%$ for crizotinib and alectinib, respectively. Notably, 37 $48 \%$ of patients did not reach the efficacy thresholds and could potentially benefit from a higher dose of crizotinib or alectinib.

Chapter 5 described exposure-response analyses in patients with melanoma treated with the combination of BRAF- and MEK-inhibitor dabrafenib plus trametinib. In total, 140 patients were included. Although dabrafenib exposure was not related to efficacy, exposure to trametinib was related to survival, with trametinib $C_{\min } \geq 15.6 \mathrm{ng} / \mathrm{mL}$ being identified as the optimal threshold. Median PFS in patients with trametinib $C_{\min } \geq 15.6$ $\mathrm{ng} / \mathrm{mL}$ was 10.9 months, compared with 6.0 months for those with $C_{\text {min }}$ below this threshold $(p=0.06)$. Furthermore, OS was ten months longer in patients with trametinib $C_{\min }$ levels $\geq 15.6 \mathrm{ng} / \mathrm{mL}$ compared to patients with $C_{\min }$ below this threshold ( $22.8 \mathrm{vs} .12 .6$ months, $p=0.003$ ). These results implicate that a substantial subset of patients (i.e. $63 \%$ ) 
may potentially benefit from a higher dose of trametinib, provided that they would tolerate this dose increase.

Taken together, exposure was related to efficacy for four out of five studied oral targeted therapies. For these drugs, validated $C_{\min }$ thresholds were identified that can be used for PK-guided dosing. In general, interindividual variability in exposure was high. In all three studies, a substantial subset of patients was underexposed (i.e. 37-63\%) at the currently used fixed dosing regimen. PK-guided dosing may optimize treatment outcomes in these patients.

\section{PART III - PRECISION DOSING IN CLINICAL PRACTICE}

This part focused on the clinical application of precision dosing for multiple oral targeted therapies in various settings.

First of all, Chapter 6 described the protocol of the Dutch Pharmacology Oncology Group - Therapeutic Drug Monitoring (DPOG-TDM) study. This is an ongoing nationwide prospective protocol providing a framework to investigate the feasibility, tolerability and efficacy of PK-guided dosing for multiple oral targeted therapies simultaneously. Primary outcome of this trial is to halve the proportion of patients with a low exposure after 12 weeks compared with historical data. Patients can be enrolled in this study when they initiate regular treatment with one of the oral targeted therapies included in the protocol at the approved dose. PK samples are then collected 4, 8 and 12 weeks after start of treatment for most compounds, and every 12 weeks thereafter. In case of $C_{\min }$ below the predefined TDM target and manageable toxicity, a PK-guided intervention is recommended. This could include emphasizing compliance, adjusting concomitant medication due to drug-drug interactions, concomitant intake with food, optimizing the dosing schedule or dose increases.

In Chapter 7, the first (interim) results of the DPOG-TDM study were reported. Of the 386 patients that were evaluable for the primary outcome, $22.8 \%$ had a low exposure after 12 weeks, compared with $40.4 \%$ in historical data $(p<0.001)$. PK-guided dosing thus reduced the proportion of underexposed patients by $44 \%$ (95\% Cl: $32-54 \%)$. Of the 543 patients that were evaluable for the overall analyses, $48.1 \%$ had a $C_{\min }$ below the target at a certain time point during treatment. In $53.3 \%$ of these patients, PK-guided interventions were performed, which were successful in $76.6 \%$ of the patients in whom the effect was evaluated. Reasons why PK-guided interventions could not be performed in other patients were mainly toxicity (63.9\%), lack of physician adherence (17.2\%) or treatment discontinuation (13.1\%). This study showed that PK-guided dose optimization of oral targeted therapies was feasible in clinical practice and reduced the proportion of underexposed patients. Therefore, these findings support the introduction of PK-guided dosing as standard of care.

Chapter 8 evaluated PK-guided dose increases of imatinib in patients with gastrointestinal stromal tumors (GIST) in routine clinical practice. Based on the strong rationale, PK-guided dosing of imatinib has been gradually implemented as standard of 
care at the Netherlands Cancer Institute since 2009. In this chapter, 169 patients were described of whom 1402 PK samples were collected. In $75 \%$ of patients, $C_{\text {min }}$ was below the efficacy threshold of $1100 \mathrm{ng} / \mathrm{mL}$. PK-guided dose increases were performed in $62 \%$ of these patients and were successful in $63 \%$ of them. PFS was similar in patients with and without imatinib dose increases. However, due to the small number of patients with progressive disease, no definite conclusions on the effect of PFS could yet be drawn. This is the largest cohort in which PK-guided dose increases of imatinib in patients with GIST were performed in routine clinical practice. These findings highlight the feasibility of PKguided dosing of imatinib in clinical practice.

Chapter 9 described two patients with soft tissue sarcoma who were treated at an eight times lower than standard dose of pazopanib due to toxicity, and still maintained a $\mathrm{C}_{\text {min }}$ above the efficacy threshold of $20.5 \mathrm{mg} / \mathrm{L}$. In the absence of PK-guided dosing, when doses would have been reduced according to the drug label, treatment would definitely have been discontinued in these patients, as the label considers the exposure at a four times lower than standard dose insufficient to obtain a clinically relevant effect. Here, the strong value of measuring pazopanib concentrations in case of dose reductions due to toxicity is illustrated, as exposure could still be adequate at considerably lower than standard doses.

Lastly, the relevant clinical pharmacokinetic and pharmacodynamic characteristics of oral anti-hormonal drugs were outlined and translated into practical guidelines for PK-guided dosing in Chapter 10. For some compounds, targets for PK-guided dosing are not well established yet and as a reference the median pharmacokinetic exposure could be targeted (i.e. exemestane and enzalutamide). However, for most drugs, exposure-efficacy relationships could be translated into specific $C_{\text {min }}$ thresholds (i.e. abiraterone: $8.4 \mathrm{ng} / \mathrm{mL}$, anastrozole: $34.2 \mathrm{ng} / \mathrm{mL}$, letrozole: $85.6 \mathrm{ng} / \mathrm{mL}$ ). Moreover, prospective clinical trials have shown PK-guided dosing to be feasible for tamoxifen, for which the efficacy threshold of its active metabolite endoxifen is suggested to be $5.97 \mathrm{ng} / \mathrm{mL}$. Based on the available data, it was therefore concluded that precision dosing based on drug concentrations is feasible and promising for oral anti-hormonal drugs and should be developed further and implemented into clinical practice.

\section{PART IV - ALTERNATIVE STRATEGIES FOR PRECISION DOSING}

In the last part of this thesis, alternative strategies that can be applied to optimize exposure were highlighted, focusing on cost-neutral interventions, drug-drug interactions and bioavailability.

Chapter 11 reported the results of the first 32 evaluable patients treated with abiraterone acetate in the DPOG-TDM study. According to the drug label, abiraterone acetate should be administered in modified fasting state, which means no food two hours before and one hour after drug intake. However, concomitant intake with food has been shown to result in a clinically relevant increase in exposure in previous food-effect studies. Therefore, the aim was to evaluate whether PK-guided dosing of abiraterone using a food 
intervention is feasible in clinical practice and results in an increased percentage of patients with efficacious exposure to abiraterone without additional toxicities. Concomitant intake with a light meal or a snack resulted in a 2.9 -fold increase in $C_{\min }$ and led to an adequate exposure in $87.5 \%$ of patients. Although concomitant intake with food also resulted in an increased interindividual variability, and thus very high $\mathrm{C}_{\min }$ levels in some patients, no additional toxicities were experienced by these patients. It was thus concluded that concomitant intake with food offers a cost-neutral opportunity to optimize exposure in patients with low abiraterone $C_{\text {min }}$.

In Chapter 12, another cost-neutral strategy was applied to optimize pazopanib exposure. At the approved fixed dose of $800 \mathrm{mg}$ once daily, $16-30 \%$ of patients treated with pazopanib are being underdosed and are at risk of decreased efficacy, providing a strong rationale for PK-guided dosing. PK-guided dosing of pazopanib has previously been proven feasible and resulted in an increased proportion of patients with adequate exposure. To achieve this, pazopanib dosages needed to be increased to 1000-1800 mg. However, due to the non-linear absorption of pazopanib, which is plateauing at dosages above $800 \mathrm{mg}$, absolute dose increments are not an efficient strategy to increase exposure. Furthermore, it leads to an increase in treatment costs. A previous population pharmacokinetic model estimated that the relative bioavailability of pazopanib dosed at $400 \mathrm{mg}$ would be $59 \%$ higher than at $800 \mathrm{mg}$. Therefore, a prospective pharmacokinetic cross-over trial was performed comparing the pharmacokinetics of pazopanib $800 \mathrm{mg}$ once daily with pazopanib $400 \mathrm{mg}$ twice daily. In this study, splitting intake moments of pazopanib resulted in a $79 \%$ increase in $\mathrm{C}_{\min }$, with acceptable tolerability and thereby provides a promising cost-neutral strategy to optimize treatment in the substantial subset of patients with a low pazopanib exposure.

As a next step, splitting intake moments of pazopanib was implemented as a PK-guided intervention in the DPOG-TDM study. The results of this cohort were reported in Chapter 13 of this thesis. In case of $C_{\min }$ below the efficacy threshold of $20.5 \mathrm{mg} / \mathrm{L}$ and manageable toxicity, intake moments were split into $400 \mathrm{mg}$ twice daily as a first step. Secondly, concomitant intake with food was recommended. By applying this PK-guided dosing strategy, the proportion of patients with a low exposure after 12 weeks was $12.9 \%$ (95\% Cl: 3.6-29.8\%), compared with $26.7 \%$ in historical data. Overall, $37 \%$ of patients had a $\mathrm{C}_{\min }$ below the target at a certain time point during treatment. In $82 \%$ of these patients, PKguided interventions could be performed, which were successful in $62 \%$ of them. Hence, it was concluded that PK-guided dose optimization of pazopanib using cost-neutral interventions is feasible in clinical practice and results in an increased proportion of patients with an adequate exposure.

Chapter 14 served as an introduction to the clinical drug-drug interaction study described in the next chapter. Here, an overview of the clinical pharmacokinetic and pharmacodynamic characteristics of cyclin-dependent kinase 4 and 6 (CDK4/6) inhibitors 
palbociclib, ribociclib and abemaciclib was provided. The results of food-effect and drugdrug interaction studies are summarized and the current knowledge on exposureresponse and exposure-toxicity relationships is highlighted. All three CDK4/6 inhibitors are characterized by a high interindividual variability in exposure, have their brain penetration limited by efflux transporters, and are extensively metabolized by cytochrome P450 3A4 (CYP3A4). Their exposure is dramatically affected by strong CYP3A4 modulators. Higher exposure is associated with an increased risk of neutropenia for all CDK4/6 inhibitors. In addition, an exposure-efficacy relationship has been demonstrated for abemaciclib, whereas these remain inconclusive thus far for palbociclib and ribociclib.

Despite the well-established effects of strong CYP3A4 modulators on the pharmacokinetics of CDK4/6 inhibitors, the effect of moderate CYP3A4 inhibitors has thus far only been studied in physiologically based pharmacokinetic simulations. Chapter 15 therefore described the preliminary results of a randomized pharmacokinetic cross-over trial in which the effect of the moderate CYP3A4 inhibitor erythromycin on the pharmacokinetics of palbociclib is studied in breast cancer patients. It was found that the area under the plasma-concentration time curve (AUC) and maximum plasma concentration $\left(C_{\max }\right)$ were increased by $44 \%$ and $43 \%$, respectively, suggesting that a dose reduction of palbociclib from $125 \mathrm{mg}$ to $75 \mathrm{mg}$ once daily would be rational, when used concomitantly with moderate CYP3A4 inhibitors. Moreover, this study could serve as a showcase for other oral targeted therapies metabolized by CYP3A4 and other moderate CYP3A4 inhibitors.

An important factor in the high variability in exposure of oral targeted therapies is a suboptimal bioavailability. Hence, the assessment of the absolute bioavailability is crucial in the development of optimized oral formulations. In the study described in Chapter 16, an innovative design was applied to determine the absolute bioavailability of oral imatinib using a stable isotopically labeled intravenous imatinib-d8 microdose. Median absolute bioavailability at steady-state was $76 \%$, which was less than the $98 \%$ reported using a traditional two-period cross-over design in healthy volunteers. This study demonstrated the potential of using a stable isotopically labeled microdose for the assessment of the absolute bioavailability. Hopefully, this new approach will result in increased and more relevant knowledge on the pharmacokinetics of novel oral targeted therapies in an early stage of drug development.

In conclusion, this thesis described the results of several studies on precision dosing of oral targeted therapies in oncology. It was concluded that there is a strong rationale for precision dosing and that randomized trials may not be necessary to demonstrate its clinical relevance. In addition, exposure was related to efficacy for many oral targeted therapies. Furthermore, PK-guided dosing was feasible in clinical practice for most compounds and cost-neutral strategies can be applied to optimize exposure for some of them. Lastly, treatment with oral targeted therapies could be further optimized by 
studying drug-drug interactions and bioavailability. When taken together, precision dosing has the potential to further optimize treatment with oral anticancer drugs and should, therefore, be implemented in routine clinical management. 



\section{Nederlandse samenvatting}





\section{NEDERLANDSE SAMENVATTING}

De komst van orale doelgerichte therapieën binnen de oncologie wordt gezien als een belangrijke doorbraak in de behandeling van kanker. Terwijl we ons steeds meer richten op behandelen op maat, door het selecteren van de juiste behandeling op basis van moleculaire kenmerken van de tumor, worden de meeste van deze nieuwe antikanker medicijnen nog steeds toegediend in een vaste dosering die voor alle patiënten gelijk is. De farmacokinetische (PK) kenmerken van deze nieuwe orale doelgerichte therapieën suggereren echter dat doseren op maat veel logischer zou zijn. Doseren op maat kan worden bereikt door het selecteren van de juiste startdosering voor iedere individuele patiënt(engroep), het zogeheten "right-dose-first-time" paradigma, waarna deze dosering verder kan worden bijgesteld door middel van PK-gestuurd doseren, dat wil zeggen het aanpassen van de dosering op basis van gemeten geneesmiddelconcentraties. Dit proefschrift richtte zich op de rationale (Deel I), het bewijs (Deel II) en de klinische toepassing (Deel III en IV) van doseren op maat van orale doelgerichte therapieën binnen de oncologie.

\section{DEEL I - RATIONALE VOOR DOSEREN OP MAAT}

Het doel van Hoofdstuk 1 was om het wetenschappelijke bewijs voor PK-gestuurd doseren van orale doelgerichte therapieën te bespreken door onderstaande criteria voor rationale toepassing ervan te bediscussiëren:

1. ontbreken van een eenvoudig meetbare biomarker voor het effect van een geneesmiddel;

2. langdurige therapie;

3. beschikbaarheid van een gevalideerde en sensitieve bio-analytische methode;

4. belangrijke mate van variabiliteit in farmacokinetische blootstelling;

5. smalle therapeutische breedte;

6. vastgestelde en consistente relaties tussen blootstelling en respons;

7. haalbare strategieën voor dosisaanpassingen.

Voor de meeste orale doelgerichte antikanker medicijnen wordt aan deze vereisten voldaan. Om het volledige potentieel van behandelen op maat te realiseren, zouden we patiënten daarom niet alleen met het juiste geneesmiddel moeten behandelen, maar ook met de juiste dosering.

Alhoewel PK-gestuurd doseren rationeel is en wordt ondersteund door een toenemende mate van bewijs, wordt het beperkt toegepast binnen de oncologie. Een argument dat vaak wordt gebruikt tegen de implementatie van PK-gestuurd doseren in de reguliere zorg is het ontbreken van gerandomiseerde gecontroleerde studies (RCT's). In Hoofdstuk 2 werd beargumenteerd waarom RCT's niet nodig zijn om de klinische relevantie van doseren op maat aan te tonen. Allereerst werden de beperkingen van de huidige vaste 
dosering en de vooruitgang tot nu toe met het implementeren van doseren op maat besproken. Vervolgens werden argumenten besproken waarom grote bevestigende RCT's niet noodzakelijk zijn voor de implementatie van PK-gestuurd doseren wanneer duidelijke relaties tussen blootstelling en effectiviteit zijn aangetoond. Tenslotte werd een plan van aanpak voorgesteld om het belang van PK-gestuurd doseren binnen de oncologie aan te tonen.

\section{DEEL II - BLOOTSTELLING-RESPONS ANALYSES}

Voordat PK-gestuurd doseren kan worden toegepast in de klinische praktijk, moeten relaties tussen blootstelling en effectiviteit worden vastgesteld en moeten optimale streefwaardes voor PK-gestuurd doseren worden geïdentificeerd. Daarom is het van essentieel belang dat blootstelling-respons analyses worden uitgevoerd. In dit deel van het proefschrift werden blootstelling-respons analyses beschreven voor vijf orale doelgerichte therapieën: abiraterone, crizotinib, alectinib, dabrafenib en trametinib.

In Hoofdstuk 3 werden blootstelling-respons analyses voor abiraterone en de belangrijkste metabolieten beschreven. Abiraterone is een remmer van 17ahydroxylase/C17,20-lyase (CYP17) en wordt gebruikt bij de behandeling van gemetastaseerd prostaatcarcinoom. In deze observationele studie zijn 62 patiënten geïncludeerd. Hierin werd bevestigd dat de eerder vastgestelde streefwaarde van een dalspiegel $\left(C_{\min }\right) \geq 8.4 \mathrm{ng} / \mathrm{mL}$ gerelateerd is aan een langere progressievrije overleving in de klinische praktijk (16.9 vs. 6.1 maanden, $p=0.033$ ). De variabiliteit in blootstelling tussen patiënten op de huidige vaste dosering was groot, met een variatie coëfficiënt van $70 \%$ voor $\mathrm{C}_{\text {min }}$. Als gevolg daarvan heeft $42 \%$ van de patiënten die behandeld worden met abiraterone acetaat in de klinische praktijk een te lage blootstelling en lopen daarmee risico op suboptimale effectiviteit van de behandeling.

Hoofdstuk 4 richtte zich op de anaplastisch lymfoom kinase (ALK)-remmers crizotinib en alectinib, die beide worden gebruikt bij de behandeling van ALK-positief gemetastaseerd niet-kleincellig longcarcinoom. Blootstelling-respons relaties werden onderzocht door gebruik te maken van eerder voorgestelde streefwaarden voor $C_{\min }$, namelijk $235 \mathrm{ng} / \mathrm{mL}$ voor crizotinib en $435 \mathrm{ng} / \mathrm{mL}$ voor alectinib. Bij 52 patiënten die behandeld werden met alectinib was de progressievrije overleving significant langer in patiënten met een mediane $C_{\min } \geq 435 \mathrm{ng} / \mathrm{mL}$, vergeleken met patiënten met een blootstelling onder deze streefwaarde (niet bereikt (95\% betrouwbaarheidsinterval: 19.8 - niet bereikt) vs. 12.6 maanden, $p=0.04)$. Voor crizotinib werd ook een klinisch relevant verschil in progressievrije overleving gevonden, alhoewel dit verschil alleen statistisch significant was in een samengestelde analyse waarin 79 patiënten werden meegenomen met ALKpositieve, ROS1-positieve en CMET-gedysreguleerde tumoren (11.8 vs. 5.3 maanden, $p=0.04)$. Op de standaarddosering was de variabiliteit in blootstelling tussen patiënten groot, met variatiecoëfficiënten voor $C_{\min }$ van $45 \%$ en $57 \%$ voor respectievelijk crizotinib 
en alectinib. Het was opmerkelijk dat 37-48\% van de patiënten de streefwaarden voor effectiviteit niet haalde. Deze patiënten zouden dus baat kunnen hebben bij een hogere dosering van crizotinib of alectinib.

Hoofdstuk 5 beschreef blootstelling-respons analyses in patiënten met melanoom die behandeld werden met de combinatie van de BRAF-remmer dabrafenib en de MEKremmer trametinib. In totaal werden 140 patiënten geïncludeerd. Alhoewel de blootstelling aan dabrafenib niet gerelateerd was aan de effectiviteit, werd wel een relatie tussen blootstelling en overleving gevonden voor trametinib, waarbij een trametinib $C_{\text {min }}$ $\geq 15.6 \mathrm{ng} / \mathrm{mL}$ werd vastgesteld als optimale streefwaarde. De mediane overleving was tien maanden langer in patiënten met een trametinib $C_{\min } \geq 15.6 \mathrm{ng} / \mathrm{mL}$ vergeleken met patiënten met een $C_{\text {min }}$ onder deze streefwaarde ( 22.8 vs. 12.6 maanden, $p=0.003$ ). De mediane progressievrije overleving in patiënten met een trametinib $C_{\min } \geq 15.6 \mathrm{ng} / \mathrm{mL}$ was 10.9 maanden, vergeleken met 6.0 maanden in patiënten met een $C_{\text {min }}$ onder deze streefwaarde $(p=0.06)$. Deze resultaten impliceren dat een substantieel deel van de patiënten (namelijk 63\%) mogelijk baat heeft bij een hogere dosering van trametinib, mits zij deze dosisverhoging verdragen.

Bij elkaar genomen was de blootstelling gerelateerd aan de effectiviteit voor vier van de vijf onderzochte orale doelgerichte therapieën. Voor deze geneesmiddelen zijn gevalideerde $C_{\min }$ streefwaarden vastgesteld die gebruikt kunnen worden voor PKgestuurd doseren. In het algemeen was de variabiliteit in blootstelling tussen patiënten groot. In alle drie de studies had een substantieel deel van de patiënten een te lage blootstelling (namelijk 37-63\%) op de huidige vaste dosering. PK-gestuurd doseren kan mogelijk de behandeluitkomsten optimaliseren in deze patiënten.

\section{DEEL III - DOSEREN OP MAAT IN DE KLINISCHE PRAKTIJK}

Dit deel richtte zich op de klinische toepassing van doseren op maat voor meerdere orale doelgerichte therapieën in verschillende situaties.

Allereerst werd in Hoofdstuk 6 het protocol van de Dutch Pharmacology Oncology Group - Therapeutic Drug Monitoring (DPOG-TDM) studie beschreven. Dit is een lopende prospectieve studie in meerdere ziekenhuizen in Nederland, waarin de haalbaarheid, veiligheid en effectiviteit van PK-gestuurd doseren wordt onderzocht voor meerdere orale doelgerichte therapieën. Het primaire eindpunt van deze studie is om de fractie patiënten met een te lage blootstelling na 12 weken te halveren in vergelijking met historische data. Patiënten kunnen in deze studie worden geïncludeerd wanneer zij starten met reguliere behandeling met een van de orale doelgerichte therapieën die zijn opgenomen in het protocol op de geregistreerde dosering. PK samples worden vervolgens afgenomen 4, 8 en 12 weken na start van de behandeling voor de meeste geneesmiddelen, en daarna iedere 12 weken. In het geval van $C_{\min }$ onder de vooraf vastgestelde TDM streefwaarde en 
dat de therapie goed verdragen wordt, wordt een PK-gestuurde interventie aangeraden. Dit kan bestaan uit het benadrukken van de therapietrouw, aanpassen van co-medicatie in het geval van geneesmiddelinteracties, gelijktijdig innemen met voedsel, optimaliseren van het doseerschema of verhogen van de dosering.

In Hoofdstuk 7 werden de eerste (interim) resultaten van de DPOG-TDM studie gerapporteerd. Van de 386 patiënten die evalueerbaar waren voor het primaire eindpunt, had $22.8 \%$ een lage blootstelling na 12 weken, vergeleken met $40.4 \%$ in historische data $(p<0.001)$. PK-gestuurd doseren heeft het aandeel patiënten met een lage blootstelling dus teruggebracht met 44\% (95\% betrouwbaarheidsinterval: 32-54\%). Van de 543 patiënten die evalueerbaar waren voor de algemene analyses, had $48.1 \%$ op enig moment tijdens de behandeling een $C_{\min }$ onder de streefwaarde. Bij 53.3\% van deze patiënten werd een PK-gestuurde interventie toegepast, welke succesvol was bij $76.6 \%$ van de patiënten bij wie het effect was geëvalueerd. Redenen waarom PK-gestuurde interventies niet konden worden toegepast bij andere patiënten waren voornamelijk toxiciteit (63.9\%), niet opvolgen van het doseeradvies door de behandelend arts (17.2\%) of staken van de behandeling (13.1\%). Deze studie heeft laten zien dat PK-gestuurde dosisoptimalisatie van orale doelgerichte therapieën haalbaar is in de klinische praktijk. Daarnaast leidde het tot een reductie van het aantal patiënten met een te lage blootstelling. Daarom ondersteunen deze bevindingen de introductie van PK-gestuurd doseren als standaardzorg.

Hoofdstuk 8 evalueerde PK-gestuurde dosisverhogingen van imatinib bij patiënten met gastro-intestinale stromale tumoren (GIST) in de reguliere patiëntenzorg. Op basis van de sterke rationale is PK-gestuurd doseren van imatinib geleidelijk geïmplementeerd als standaardzorg in het Antoni van Leeuwenhoek sinds 2009. In dit hoofdstuk werden 169 patiënten beschreven van wie 1402 PK samples zijn verzameld. Bij $75 \%$ van deze patiënten was de $C_{\min }$ onder de streefwaarde voor effectiviteit van $1100 \mathrm{ng} / \mathrm{mL}$. PK-gestuurde dosisverhogingen zijn toegepast in $62 \%$ van deze patiënten en waren succesvol bij $63 \%$ van hen. De progressievrije overleving was vergelijkbaar tussen patiënten met en zonder dosisverhogingen van imatinib. Door het kleine aantal patiënten met progressieve ziekte konden echter geen definitieve conclusies worden getrokken over het effect op progressievrije overleving. Dit is het grootste cohort waarin PK-gestuurde dosisverhogingen van imatinib zijn beschreven bij patiënten met GIST. Deze bevindingen benadrukken de haalbaarheid van PK-gestuurd doseren van imatinib in de klinische praktijk.

Hoofdstuk 9 beschreef twee patiënten met een wekedelensarcoom die werden behandeld op een acht keer lagere dosering dan de standaard dosering van pazopanib vanwege toxiciteit en toch een $C_{\min }$ boven de streefwaarde van $20.5 \mathrm{mg} / \mathrm{L}$ behielden. Zonder PK-gestuurd doseren, wanneer de dosering gereduceerd zou zijn volgens de bijsluiter, zou de behandeling zeker gestaakt zijn bij deze patienten, omdat de 
blootstelling op een vier keer lagere dosering dan de standaard dosering in de bijsluiter wordt beschouwd als onvoldoende om een klinisch relevant effect te bewerkstelligen. Hier werd de sterke meerwaarde geillustreerd van het meten van pazopanib concentraties in het geval van dosisreducties vanwege toxiciteit, omdat de blootstelling toch adequaat kan zijn op aanzienlijk lagere doseringen dan de standaard dosering.

Tot slot werden de relevante klinisch farmacokinetische en farmacodynamische kenmerken van orale anti-hormonale geneesmiddelen samengevat en omgezet in praktische richtlijnen voor PK-gestuurd doseren in Hoofdstuk 10. Voor sommige geneesmiddelen zijn streefwaarden voor PK-gestuurd doseren nog niet goed vastgesteld en als referentie kan de mediane PK blootstelling worden nagestreefd (dit is het geval voor exemestaan en enzalutamide). Voor de meeste geneesmiddelen konden blootstelling-respons relaties echter worden omgezet in specifieke $C_{\min }$ streefwaarden (dit is het geval voor abiraterone: $8.4 \mathrm{ng} / \mathrm{mL}$, anastrozol: $34.2 \mathrm{ng} / \mathrm{mL}$ en letrozol: $85.6 \mathrm{ng} / \mathrm{mL}$ ). Bovendien hebben prospectieve klinische studies laten zien dat PK-gestuurd doseren haalbaar is voor tamoxifen, waarvoor een streefwaarde voor effectiviteit van de actieve metaboliet endoxifen van $5.97 \mathrm{ng} / \mathrm{mL}$ wordt voorgesteld. Op basis van de beschikbare data wordt daarom geconcludeerd dat doseren op mat op basis van geneesmiddelconcentraties haalbaar en veelbelovend is voor orale anti-hormonale geneesmiddelen. Dit moet nu verder ontwikkeld worden en geïmplementeerd worden in de klinische praktijk.

\section{DEEL IV - ALTERNATIEVE STRATEGIEËN VOOR DOSEREN OP MAAT}

In het laatste deel van dit proefschrift werden alternatieve strategieën uitgelicht die toegepast kunnen worden voor het optimaliseren van de blootstelling, toegespitst op kostenneutrale interventies, geneesmiddelinteracties en biologische beschikbaarheid.

Hoofdstuk 11 rapporteerde de resultaten van de eerste 32 patiënten die behandeld zijn met abiraterone binnen de DPOG-TDM studie. Volgens de bijsluiter moet abiraterone acetaat worden ingenomen in gemodificeerde nuchtere toestand, wat inhoudt dat er geen voedsel twee uur voor en één uur na inname van het geneesmiddel gebruikt mag worden. In eerdere voedsel-effect studies is echter aangetoond dat gelijktijdige inname met voedsel resulteert in een klinisch relevante toename van de blootstelling. Daarom was het doel van deze studie om te evalueren of PK-gestuurd doseren van abiraterone met behulp van voedsel interventies haalbaar is in de klinische praktijk en of dit resulteert in een hoger percentage patiënten met een effectieve blootstelling aan abiraterone zonder extra toxiciteit. Gelijktijdige inname met een lichte maaltijd of een tussendoortje resulteerde in een 2.9-voudige toename in $C_{\min }$ en leidde tot een adequate blootstelling bij $87.5 \%$ van de patiënten. Alhoewel gelijktijdige inname met voedsel ook resulteerde in een grotere variabiliteit tussen patiënten, en dus een erg hoge $C_{\min }$ in sommige patiënten, ervaarden deze patiënten niet meer bijwerkingen. Daarom werd geconcludeerd dat 
gelijktijdige inname met voedsel een kostenneutrale mogelijkheid biedt om de blootstelling te optimaliseren voor patiënten met een lage abiraterone $C_{\text {min }}$.

In Hoofdstuk 12 werd een andere kostenneutrale strategie toegepast om de blootstelling aan pazopanib te optimaliseren. Op de geregistreerde vaste dosering van eenmaal daags $800 \mathrm{mg}$ heeft $16-30 \%$ van de patiënten die behandeld worden met pazopanib een te lage blootstelling en lopen dus risico op een verminderde effectiviteit. Daarom is er een sterke rationale voor PK-gestuurd doseren. Eerder is aangetoond dat PK-gestuurd doseren van pazopanib haalbaar is en resulteert in een toename van het aantal patiënten met een adequate blootstelling. Om dit te bereiken was het nodig om de pazopanib doseringen te verhogen tot 1000-1800 mg. Door de non-lineaire opname van pazopanib, die een plateau bereikt op doseringen boven $800 \mathrm{mg}$, zijn absolute dosisverhogingen geen efficiënte strategie om de blootstelling te verhogen. Bovendien leidt dit tot een toename van de behandelkosten. Een eerder populatie farmacokinetisch model voorspelde dat de relatieve biologische beschikbaarheid van pazopanib op een dosering van $400 \mathrm{mg} 59 \%$ hoger zou zijn dan op 800 mg. Daarom werd een prospectieve farmacokinetische crossover studie uitgevoerd waarin de farmacokinetiek van eenmaal daags $800 \mathrm{mg}$ pazopanib werd vergeleken met tweemaal daags $400 \mathrm{mg}$. In deze studie resulteerde het spreiden van innamemomenten van pazopanib in een stijging van $79 \%$ in $C_{\text {min }}$, met acceptabele bijwerkingen. Daarmee biedt het een veelbelovende kostenneutrale strategie om de behandeling te optimaliseren voor het aanzienlijke deel van de patiënten met een lage blootstelling aan pazopanib.

De volgende stap was om het spreiden van innamemomenten van pazopanib te implementeren als PK-gestuurde interventie binnen de DPOG-TDM studie. De resultaten van dit cohort werden gerapporteerd in Hoofdstuk 13 van dit proefschrift. In het geval van $C_{\min }$ onder de streefwaarde van $20.5 \mathrm{mg} / \mathrm{L}$ en acceptabele toxiciteit werden de inname momenten gesplitst in tweemaal daags $400 \mathrm{mg}$ als eerste stap. Als tweede stap werd gelijktijdige inname met voedsel aangeraden. Door het toepassen van deze PK-gestuurde doseerstrategie, was het percentage patiënten met een lage blootstelling na 12 weken 12.9\% (95\% betrouwbaarheidsinterval: 3.6-29.8\%), vergeleken met $26.7 \%$ in historische data. Daarnaast had 37\% van de patiënten op een zeker moment tijdens de behandeling een $C_{\text {min }}$ onder de streefwaarde. In $82 \%$ van deze patiënten kon een PK-gestuurde interventie worden toegepast, die succesvol was in $62 \%$ van hen. Daarom werd geconcludeerd dat PK-gestuurde dosisoptimalisatie van pazopanib door middel van kostenneutrale interventies haalbaar is in de klinische praktijk en resulteert in een toegenomen aantal patiënten met een adequate blootstelling.

Hoofdstuk 14 diende als introductie op de klinische geneesmiddelinteractie studie beschreven in het volgende hoofdstuk. Hierin werd een overzicht gegeven van klinisch farmacokinetische en farmacodynamische kenmerken van de cycline-afhankelijke kinasen 4 en 6 (CDK4/6) remmers palbociclib, ribociclib en abemaciclib. De resultaten van 
voedsel-effect en geneesmiddelinteractie studies werden samengevat en de huidige kennis over blootstelling-respons en blootstelling-toxiciteit relaties werden besproken. Alle drie de CDK4/6 remmers worden gekenmerkt door een hoge variabiliteit in blootstelling tussen patiënten, een blootstelling in de hersenen die beperkt wordt door efflux transporters en uitgebreide metabolisatie door cytochroom P450 3A4 (CYP3A4). De blootstelling aan deze geneesmiddelen wordt in grote mate beïnvloedt door sterke CYP3A4 modulatoren. Hogere blootstelling is geassocieerd met een verhoogd risico op neutropenie voor alle CDK4/6 remmers. Daarnaast is een relatie tussen blootstelling en effectiviteit aangetoond voor abemaciclib, terwijl deze analyses tot nu toe niet eenduidig waren voor palbociclib en ribociclib.

Ondanks de welbekende effecten van sterke CYP3A4 modulatoren op de farmacokinetiek van CDK4/6 remmers, is het effect van matige CYP3A4 remmers tot nu toe alleen onderzocht in fysiologisch gebaseerde PK simulaties. Hoofdstuk 15 beschreef daarom de voorlopige resultaten van een gerandomiseerde PK cross-over studie waarin het effect van de matige CYP3A4 remmer erythromycine op de PK van palbociclib wordt bestudeerd in patiënten met borstkanker. De oppervlakte onder de plasma concentratie-tijd curve (AUC) en de topspiegel $\left(C_{\max }\right)$ namen toe met respectievelijk 44\% en 43\%. Dit suggereert dat een dosisreductie van palbociclib van eenmaal daags $125 \mathrm{mg}$ naar eenmaal daags $75 \mathrm{mg}$ rationeel zou zijn bij gelijktijdig gebruik met matige CYP3A4 remmers. Bovendien kan deze studie als voorbeeld dienen voor andere orale doelgerichte therapieën die gemetaboliseerd worden door CYP3A4, en voor andere matige CYP3A4 remmers.

Een belangrijke factor in de grote variabiliteit in blootstelling van orale doelgerichte therapieën, is een suboptimale biologische beschikbaarheid. Daarom is het vaststellen van de absolute biologische beschikbaarheid cruciaal in de ontwikkeling van geoptimaliseerde orale formuleringen. In de studie beschreven in Hoofdstuk $\mathbf{1 6}$ is een innovatieve studieopzet toegepast om de absolute biologische beschikbaarheid van oraal imatinib te bepalen door middel van een stabiele isotoop gelabelde intraveneuze imatinib-d8 microdosis. De mediane absolute biologische beschikbaarheid op steady-state was $76 \%$. Dat is minder dan de $98 \%$ die gevonden is met de traditionele two-period crossover opzet bij gezonde vrijwilligers. Deze studie toont de potentie van het gebruik van een stabiele isotoop gelabelde microdosis voor het beoordelen van de absolute biologische beschikbaarheid. Hopelijk leidt deze nieuwe benadering tot een toename van meer relevante kennis van de farmacokinetiek van nieuwe orale doelgerichte therapieën in een vroeg stadium van geneesmiddelenontwikkeling.

Concluderend beschreef dit proefschrift de resultaten van verschillende studies naar doseren op maat van orale doelgerichte therapieën binnen de oncologie. Er wordt geconcludeerd dat er een sterke rationale is voor doseren op maat en dat gerandomiseerde studies niet nodig zijn om de klinische relevantie daarvan aan te tonen. Daarnaast was de blootstelling gerelateerd aan de effectiviteit voor veel orale 
doelgerichte therapieën. Bovendien was PK-gestuurd doseren haalbaar in de klinische praktijk voor de meeste geneesmiddelen en konden kostenneutrale strategieën worden toegepast om de blootstelling te optimaliseren voor sommige van deze geneesmiddelen. Tot slot kan de behandeling met orale doelgerichte therapieën nog verder worden geoptimaliseerd door onderzoek te doen naar geneesmiddelinteracties en de biologische beschikbaarheid. Bij elkaar genomen heeft doseren op maat de potentie om de behandeling met orale antikanker medicijnen verder te verbeteren, en daarom zou het geïmplementeerd moeten worden in de routine patiëntenzorg. 




\title{
Appendices
}

\author{
Author affiliations \\ List of publications \\ Dankwoord \\ Curriculum vitae
}




\section{AUTHOR AFFILIATIONS}

Jos H. Beijnen

Andries M. Bergman

Jan H. Beumer

Christian U. Blank

C. Louwrens Braal

Jacobus A. Burgers

Ingrid M.E. Desar

Vincent O. Dezentje

Marloes G.J. van Dongen

Ruben A.G. van Eerden

Nielka P. van Erp

Jeantine M. de Feijter

Dieuwertje R. Geel
Department of Pharmacy \& Pharmacology, The Netherlands Cancer Institute, Amsterdam, The Netherlands

Department of Pharmaceutical Sciences, Utrecht University, Utrecht, The Netherlands

Department of Medical Oncology, The Netherlands Cancer Institute, Amsterdam, The Netherlands

Cancer Therapeutics Program, UPMC Hillman Cancer Center, Pittsburgh, United States of America

Department of Medical Oncology, The Netherlands Cancer Institute, Amsterdam, The Netherlands

Department of Medical Oncology, Erasmus MC Cancer Institute, Rotterdam, The Netherlands

Department of Thoracic Oncology, The Netherlands Cancer Institute, Amsterdam, The Netherlands

Department of Medical Oncology, Radboud University Medical Center, Nijmegen, The Netherlands

Department of Medical Oncology, The Netherlands Cancer Institute, Amsterdam, The Netherlands

Department of Clinical Pharmacology, Division of Medical Oncology, The Netherlands Cancer Institute, Amsterdam, The Netherlands

Department of Medical Oncology, Erasmus MC Cancer Institute, Rotterdam, The Netherlands

Department of Pharmacology, Radboud University Medical Center, Nijmegen, The Netherlands

Department of Medical Oncology, The Netherlands Cancer Institute, Amsterdam, The Netherlands

Department of Pharmacy \& Pharmacology, The Netherlands Cancer Institute, Amsterdam, The Netherlands 
Hans Gelderblom

Winette T.A. van der Graaf

John B.A.G. Haanen

Alwin D.R. Huitema

Nikki S. IJzerman

Alexander L.T. Imholz

Julie M. Janssen

Markus Joerger

Daniela Katz

J. Martijn Kerst

Anne Miek Koenen

Stijn L.W. Koolen

Luc Lucas
Department of Medical Oncology, Leiden University Medical Center, Leiden, The Netherlands

Department of Medical Oncology, The Netherlands Cancer Institute, Amsterdam, The Netherlands

Department of Medical Oncology, The Netherlands Cancer Institute, Amsterdam, The Netherlands

Department of Pharmacy \& Pharmacology, The Netherlands Cancer Institute, Amsterdam, The Netherlands

Department of Clinical Pharmacy, University Medical Center Utrecht, Utrecht University, Utrecht, The Netherlands

Department of Clinical Pharmacology, Division of Medical Oncology, The Netherlands Cancer Institute, Amsterdam, The Netherlands

Department of Medical Oncology, Erasmus MC Cancer Institute, Rotterdam, The Netherlands

Department of Medical Oncology, Deventer Hospital, Deventer, The Netherlands

Department of Pharmacy \& Pharmacology, The Netherlands Cancer Institute, Amsterdam, The Netherlands

Department of Medical Oncology \& Hematology, Cantonal Hospital, St. Gallen, Switzerland

Department of Oncology, Assaf Harofeh Medical Center, Zrifin, Israel

Department of Medical Oncology, The Netherlands Cancer Institute, Amsterdam, The Netherlands

Department of Medical Oncology, The Netherlands Cancer Institute, Amsterdam, The Netherlands

Department of Pharmacy, Erasmus Medical Center, Rotterdam, The Netherlands

Department of Pharmacy \& Pharmacology, The Netherlands Cancer Institute, Amsterdam, The Netherlands 
Alejandra Martínez-Chávez

Ron H.J. Mathijssen

Dirk Jan A.R. Moes

Laura Molenaar-Kuijsten

Cynthia M. Nijenhuis

Bastiaan Nuijen

Merel van Nuland

Hans-Martin Otten

Anna K.L. Reyners

Jeroen Roosendaal

Hilde Rosing

Jan H.M. Schellens

Alfred H. Schinkel
Department of Pharmacy \& Pharmacology, The Netherlands Cancer Institute, Amsterdam, The Netherlands

Department of Medical Oncology, Erasmus MC Cancer Institute, Rotterdam, The Netherlands

Department of Clinical Pharmacy \& Toxicology, Leiden University Medical Center, Leiden, The Netherlands

Department of Pharmacy \& Pharmacology, The Netherlands Cancer Institute, Amsterdam, The Netherlands

Department of Pharmacy \& Pharmacology, The Netherlands Cancer Institute, Amsterdam, The Netherlands

Department of Pharmacy \& Pharmacology, The Netherlands Cancer Institute, Amsterdam, The Netherlands

Department of Pharmacy \& Pharmacology, The Netherlands Cancer Institute, Amsterdam, The Netherlands

Department of Medical Oncology, Meander Medical Center, Amersfoort, The Netherlands

Department of Medical Oncology, University Medical Center Groningen, Groningen, The Netherlands

Department of Pharmacy \& Pharmacology, The Netherlands Cancer Institute, Amsterdam, The Netherlands

Department of Pharmacy \& Pharmacology, The Netherlands Cancer Institute, Amsterdam, The Netherlands

Department of Clinical Pharmacology, Division of Medical Oncology, The Netherlands Cancer Institute, Amsterdam, The Netherlands

Department of Pharmaceutical Sciences, Utrecht University, Utrecht, The Netherlands

Department of Pharmacy \& Pharmacology, The Netherlands Cancer Institute, Amsterdam, The Netherlands 
Egbert F. Smit

Alex Sparreboom

Neeltje Steeghs

Johannes V. van Thienen

Bas Thijssen

Nikkie Venekamp

Remy B. Verheijen

Niels de Vries

Annelie J.E. Vulink

Kim Westerdijk

Sofie Wilgenhof
Department of Thoracic Oncology, The Netherlands Cancer Institute, Amsterdam, The Netherlands

Division of Pharmaceutics and Pharmacology, College of Pharmacy and Comprehensive Cancer Center, The Ohio State University, Columbus, United States of America

Department of Clinical Pharmacology, Division of Medical Oncology, The Netherlands Cancer Institute, Amsterdam, The Netherlands

Department of Medical Oncology, The Netherlands Cancer Institute, Amsterdam, The Netherlands

Department of Pharmacy \& Pharmacology, The Netherlands Cancer Institute, Amsterdam, The Netherlands

Department of Pharmacy \& Pharmacology, The Netherlands Cancer Institute, Amsterdam, The Netherlands

Department of Pharmacy \& Pharmacology, The Netherlands Cancer Institute, Amsterdam, The Netherlands

Department of Pharmacy \& Pharmacology, The Netherlands Cancer Institute, Amsterdam, The Netherlands

Department of Medical Oncology, Reinier de Graaf Hospital, Delft, The Netherlands

Department of Medical Oncology, Radboud University Medical Center, Nijmegen, The Netherlands

Department of Medical Oncology, The Netherlands Cancer Institute, Amsterdam, The Netherlands 


\section{LIST OF PUBLICATIONS}

\section{Peer-reviewed articles}

1. Mueller-Schoell A, Groenland SL, Scherf-Clavel O, van Dyk M, Huisinga W, Michelet R, Jaehde $U$, Steeghs N, Huitema ADR, Kloft C. Therapeutic drug monitoring of oral targeted antineoplastic drugs. Eur J Clin Pharmacol. Online ahead of print 9 Nov 2020.

2. Groenland SL*, Martínez-Chávez A*, van Dongen MGJ, Beijnen JH, Schinkel AH, Huitema ADR, Steeghs N. Clinical pharmacokinetics and pharmacodynamics of the cyclin-dependent kinase 4 and 6 inhibitors palbociclib, ribociclib, and abemaciclib. Clin Pharmacokinet. Online ahead of print 8 Oct 2020.

3. Groenland SL*, IJzerman NS*, Koenen AM, Kerst JM, van der Graaf WTA, Rosing $\mathrm{H}$, Beijnen JH, Huitema ADR, Steeghs N. Therapeutic drug monitoring of imatinib in patients with gastrointestinal stromal tumours - results from daily clinical practice. Eur J Cancer 2020; 136: 140-148.

4. Groenland SL, Geel DR, Janssen JM, de Vries N, Rosing H, Beijnen JH, Burgers JA, Smit EF, Huitema ADR, Steeghs N. Exposure-response analyses of anaplastic lymphoma kinase inhibitors crizotinib and alectinib in non-small-cell lung cancer patients. Clin Pharmacol Ther. Online ahead of print 19 Jul 2020.

5. Roosendaal J, Groenland SL, Rosing H, Lucas L, Venekamp N, Nuijen B, Huitema ADR, Beijnen JH, Steeghs N. Determination of the absolute bioavailability of oral imatinib using stable isotopically labeled intravenous imatinib-d8 microdose. Eur J Clin Pharmacol 2020; 76: 1075-1082.

6. Groenland SL, van Nuland M, Bergman AM, de Feijter JM, Dezentje VO, Rosing H, Beijnen JH, Huitema ADR, Steeghs N. Concomitant intake of abiraterone acetate and food to increase pharmacokinetic exposure: real life data from a therapeutic drug monitoring programme. Eur J Cancer 2020; 130: 32-38.

7. Groenland SL, van Eerden RAG, Verheijen RB, de Vries N, Thijssen B, Rosing $H$, Beijnen JH, Koolen SLW, Mathijssen RHJ, Huitema ADR, Steeghs N; Dutch Pharmacology Oncology Group (DPOG). Cost-neutral optimization of pazopanib exposure by splitting intake moments: a prospective pharmacokinetic study in cancer patients. Clin Pharmacokinet 2020; 59: 941-948.

8. van Nuland M, Groenland SL, Bergman AM, Steeghs N, Rosing H, Venekamp N, Huitema ADR, Beijnen JH. Exposure-response analyses of abiraterone and its metabolites in real-world patients with metastatic castration-resistant prostate cancer. Prostate Cancer Prostatic Dis 2020; 23: 244-251. 
9. Groenland SL, van Eerden RAG, Verheijen RB, Koolen SLW, Moes DJAR, Desar IME, Reyners AKL, Gelderblom HJ, van Erp NP, Mathijssen RHJ, Huitema ADR, Steeghs $\mathrm{N}$. Therapeutic drug monitoring of oral anticancer drugs: the Dutch Pharmacology Oncology Group-Therapeutic Drug Monitoring protocol for a prospective study. Ther Drug Monit 2019; 41: 561-567.

10. Groenland SL, Mathijssen RHJ, Beijnen JH, Huitema ADR, Steeghs N. Individualized dosing of oral targeted therapies in oncology is crucial in the era of precision medicine. Eur J Clin Pharmacol 2019; 75: 1309-1318.

11. van Nuland $\mathbf{M}$, Groenland SL, Bergman AM, Rotmans Jl, Rosing $\mathrm{H}$, Beijnen JH, Huitema ADR. Plasma levels of enzalutamide and its main metabolites in a patient with metastatic castration-resistant prostate cancer undergoing hemodialysis. Clin Genitourin Cancer 2019; 17: e383-e386.

12. Groenland SL, Katz D, Huitema ADR, Steeghs N. Harnessing soft tissue sarcoma with low-dose pazopanib - a matter of blood levels. BMC Cancer 2018; 18: 1200.

13. Groenland SL, van Nuland M, Verheijen RB, Schellens JHM, Beijnen JH, Huitema ADR, Steeghs N. Therapeutic drug monitoring of oral anti-hormonal drugs in oncology. Clin Pharmacokinet 2019; 58: 299-308.

14. Herbrink M, Groenland SL, Huitema ADR, Schellens JHM, Beijnen JH, Steeghs N, Nuijen B. Solubility and bioavailability improvement of pazopanib hydrochloride. Int J Pharm 2018; 544: 181-190.

15. Groenland SL*, Houben E*, van der Heijden JW, Voskuyl AE, Doodeman HJ, Penne EL. Relation between duration of the prodromal phase and renal damage in ANCA-associated vasculitis. BMC Nephrology 2017; 18: 378.

* these authors contributed equally and thus share first authorship

\section{Conference abstracts}

1. Groenland $\mathbf{S L}$, Janssen JM, Nijenhuis $C M$, de Vries $N$, Rosing $H$, Wilgenhof $S$, van Thienen JV, Haanen JBAG, Blank CU, Beijnen JH, Huitema ADR, Steeghs N. 567P Exposure-response analyses of dabrafenib and trametinib in melanoma patients. ESMO 2020 Virtual Congress.

2. Groenland SL, van Eerden RAG, Koolen SLW, Moes DJAR, Imholz A, Wilgenhof S, van der Graaf WTA, Gelderblom H, Beijnen JH, Mathijssen RHJ, Huitema ADR, Steeghs N. Therapeutic drug monitoring of pazopanib: using cost-neutral PKguided interventions to optimize exposure. ASCO 2020 Virtual Scientific Programme. 
3. Angevin E, Groenland SL, Lim AML, Liberal JM, Moreno V, Trigo JM, le Tourneau C, Mathew M, Cho DC, Hansen AR, Vicente D, Maio M, Italiano A, Bauman JR, Chisamore MJ, Zhou H, Ellis CE, Ballas MS, Hoos A, Rischin D. Updated analysis of the inducible T-cell co-stimulatory receptor (ICOS) agonist, GSK3359609 (GSK609), combination with pembrolizumab (PE) in patients (pts) with anti-PD-1/L1 treatment-naïve head and neck squamous cell carcinoma (HNSCC). ASCO 2020 Virtual Scientific Programme.

4. Goldman JW, Piha-Paul SA, Curti BD, Pedersen K, Bauer TM, Groenland SL, Carvajal RD, Chhaya V, Hammond SA, Streicher K, Townsley DM, Chae YK, Voortman J, Marabelle A, Powderley JD. Safety and tolerability of MEDI0562 in combination with durvalumab or tremelimumab in patients with advanced solid tumors. ASCO 2020 Virtual Scientific Programme.

5. Groenland SL, van Eerden RAG, Koolen SLW, Moes DJAR, Desar IME, Touw DJ, Imholz ALT, Reyners AKL, Gelderblom H, van Erp NP, Mathijssen RHJ, Huitema ADR, Steeghs N. 4420 Therapeutic drug monitoring of oral anticancer drugs preliminary results of a prospective study. ESMO 2019, Barcelona.

6. Groenland SL, Geel DR, Janssen JM, Beijnen JH, Burgers SA, Smit EF, Huitema ADR, Steeghs N. 1486PD Exposure-response analyses of ALK-inhibitors crizotinib and alectinib in NSCLC patients. ESMO 2019, Barcelona.

7. Rischin D, Groenland SL, Lim AML, Martin-Liberal J, Moreno V, Trigo Perez JM, Le Tourneau C, Mathew M, Cho DC, Hansen AR, Vincente-Baz D, Maio M, Italiano A, Bauman JR, Chisamore M, Zhou H, Ellis C, Ballas M, Hoos A, Angevin E. 1119PD Inducible T cell costimulatory (ICOS) receptor agonist, GSK3359609 (GSK609) alone and in combination with pembrolizumab (pembro): preliminary results from INDUCE-1 expansion cohorts (EC) in head and neck squamous cell carcinoma (HNSCC). ESMO 2019, Barcelona.

8. Maio M, Groenland SL, Bauer T, Rischin D, Gardeazabal I, Moreno V, Trigo Perez JM, Chisamore M, Sadik Shaik J, Rigat F, Ellis C, Chen H, Gagnon R, Scherer S, Turner D, Yadavilli S, Ballas M, Hoos A, Angevin E. 1971P Pharmacokinetic/ pharmacodynamic (PK/PD) exposure-response characterization of GSK3359609 (GSK609) from INDUCE-1, a phase I open-label study. ESMO 2019, Barcelona.

9. Groenland SL, van Eerden RAG, Verheijen RB, Huitema ADR, Mathijssen RHJ, Steeghs N. Boosting pazopanib exposure by splitting intake moments - a prospective pharmacokinetic study in cancer patients. ASCO 2019, Chicago.

10. Groenland SL, Bergman AM, Huitema ADR, Steeghs N. Concomitant intake of abiraterone and food to increase pharmacokinetic exposure: real life data from a therapeutic drug monitoring program. ASCO 2019, Chicago. 
11. Groenland SL, van Eerden RAG, Verheijen RB, Huitema ADR, Mathijssen RHJ, Steeghs N. Boosting pazopanib exposure by splitting intake moments - a prospective pharmacokinetic study in cancer patients. NVKF\&B Scientific Meeting 2019, The Netherlands.

12. Geel DR, Groenland SL, Burgers JA, Beijnen JH, Huitema ADR, Steeghs N. Exposure-response analysis of ALK-inhibitor crizotinib in non-small cell lung cancer patients. NVKF\&B Scientific Meeting 2019, The Netherlands.

13. Groenland SL, Sanchez-Spitman AB, Moes DJAR, Koolen SL, Dezentje VO, van Erp NP, Mathijssen RHJ, Guchelaar H, Huitema ADR, Steeghs N. 258P Incidence of clinically significant toxicities in patients with high endoxifen concentrations. ESMO 2018, Munich.

14. Hansen A, Bauer TM, Moreno V, Maio M, Groenland SL, Martin-Liberal J, Gan H, Rischin D, Millward M, Olszanski AJ, Cho DC, Paul E, Ballas M, Ellis C, Zhou H, Yadavilli S, Sadik Shaik J, Schmidt EV, Hoos A, Angevin E. 1138PD First in human study with GSK3359609 [GSK609], inducible T cell co-stimulator (ICOS) receptor agonist in patients [pts] with advanced, solid tumors: preliminary results from INDUCE-1. ESMO 2018, Munich.

15. Groenland SL, Bergman AM, Huitema ADR, Steeghs N. Therapeutic drug monitoring of abiraterone: concomitant intake with food increases exposure in patients with low plasma concentrations. FIGON Dutch Medicine Days 2018, The Netherlands.

16. Groenland SL, van Eerden RAG, Verheijen RB, Huitema ADR, Mathijssen RHJ, Steeghs N. Increasing pazopanib exposure by splitting intake moments. NVKF\&B Scientific Meeting 2018, The Netherlands.

17. Herbrink M, Groenland SL, Steeghs N, Huitema ADR, Schellens JHM, Beijnen JH, Nuijen B. solubility and bioavailability improvement of pazopanib hydrochloride. AAPS Annual Meeting 2017, San Diego.

18. Groenland SL, Houben E, van der Heijden JW, Voskuyl AE, Doodeman HJ, Penne EL. Relation between duration of the prodromal phase and renal damage in ANCA-associated vasculitis. Nefrologiedagen 2017, The Netherlands.

19. Schutte T, Tichelaar J, Reumerman MO, van Eekeren R, Groenland SL, Roffes L, Richir MC, van Puijenbroek E, van Agtmael MA. Student views on the student-run pharmacovigilance program. EACPT 2015, Madrid. 


\section{DANKWOORD}

Dat was het dan! Nog als de dag van gisteren herinner ik mij hoe ik het AVL binnen stapte op mijn eerste werkdag. De afgelopen vier jaar zijn voorbij gevlogen, met dit proefschrift als eindresultaat. Ergens was ik bang dat ik fulltime onderzoek doen misschien saai zou gaan vinden, maar ik heb me geen moment verveeld. Het was een fantastische tijd en ik vond het ontzettend leuk om aan dit onderzoek te mogen werken. Dit alles was natuurlijk niet mogelijk geweest zonder de inzet, hulp en steun van velen, en ik wil iedereen die hieraan heeft bijgedragen dan ook heel erg bedanken.

Allereerst alle patiënten die hebben meegedaan aan de klinische studies beschreven in dit proefschrift. Ik vind het heel knap hoe zij in deze moeilijke tijd een bijdrage hebben willen leveren aan de wetenschap, zonder hen was dit onderzoek niet mogelijk geweest.

Neeltje, bedankt dat je mij de kans hebt gegeven om aan dit avontuur te beginnen. Ik waardeer het enorm dat je altijd tijd maakt in jouw drukke agenda om mijn protocollen en manuscripten snel van commentaar te voorzien en om met me mee te denken over oplossingen. Bedankt ook dat je mij de vrijheid hebt gegeven om me te ontwikkelen tot een zelfstandige onderzoeker. Ik vind het heel knap hoe jij het overzicht houdt over de vele onderzoeksprojecten in combinatie met jouw klinische taken, en op beide gebieden altijd gestructureerd te werk gaat. Hier heb ik veel van geleerd.

Alwin, jouw enthousiasme voor klinisch farmacologisch onderzoek heeft aanstekelijk gewerkt. Bedankt voor het bijspijkeren van mijn kennis over farmacokinetiek. Je hebt me geleerd hoe ik onderzoek kan doen met een pragmatische instelling en om met oplossingen te komen in plaats van met problemen. Jouw onvoorwaardelijke steun door de jaren heen en bij het afronden van dit proefschrift hebben me enorm geholpen.

Jos, jouw feedback op manuscripten is goud waard. Ik vind het knap hoe jij stukken zowel inhoudelijk als qua vorm van uitstekend en gedetailleerd commentaar voorziet. Hier heb ik veel van geleerd en dat is de manuscripten in dit proefschrift zeker ten goede gekomen.

Frans, bedankt dat je me de mogelijkheid hebt gegeven om naast mijn promotieonderzoek ook de opleiding tot klinisch farmacoloog te volgen. Ik heb veel geleerd van alle casuïstiek en klinische voorbeelden die je met ons deelt.

Zonder het bioanalytisch laboratorium van de apotheek was dit onderzoek niet mogelijk geweest. Voor dit proefschrift hebben jullie de geneesmiddelconcentraties in maar liefst 5465 samples gemeten. In het bijzonder wil ik Niels, Bas, Hilde en de TDM analisten hiervoor bedanken. Ook het TDM-team waarmee we wekelijks alle spiegels rapporteren ben ik veel dank verschuldigd: Julie, Laura, René, David, Alaa en Lisa, ook voor het waarnemen van de TDM-studie tijdens mijn vakanties.

Veel dank ook aan alle co-auteurs van de verschillende manuscripten voor jullie hulp bij de inclusie van patiënten in de klinische studies en voor jullie kritische blik op de manuscripten. Ook iedereen die op een andere manier heeft bijgedragen aan de klinische studies wil ik daarvoor bedanken, met name Else en Carla voor jullie ondersteuning als 
CRA, Patricia en Brigitte voor het datamanagement, Ruud voor het opstellen van alle contracten, het trialbureau voor de administratieve ondersteuning, en de verpleegkundig specialisten, researchverpleegkundigen en kinetiekverpleegkundigen van de Clinical Research Unit. Bedankt voor de prettige samenwerking de afgelopen jaren! Hans, Thea en Lara, bedankt voor de goede organisatie op H3.

Alle oncologen en ziekenhuisapothekers van de Dutch Pharmacology Oncology Group wil ik bedanken voor de vele mooie klinisch farmacologische studies die we samen hebben kunnen opzetten en uitvoeren. In het bijzonder wil ik hierbij Ruben, Louwrens, Stijn en Ron uit het Erasmus MC, Kim, Nielka en Ingrid uit het Radboud UMC, en Dirk Jan en Hans uit het LUMC noemen. Ook alle andere centra die deelnemen aan de DPOGTDM studie wil ik bedanken voor hun inzet.

Dieuwertje, ik had me geen betere stagiaire kunnen wensen! Bedankt voor jouw belangrijke bijdrage aan het ALK-remmers project. Merel, maar liefst drie hoofdstukken in dit proefschrift hebben we samen geschreven. Bedankt voor de fijne en efficiënte samenwerking. Jeroen, door ons goede teamwork voor de imatinib microdosing studie hebben we deze klinische studie binnen recordtijd kunnen afronden. Nikki, het analyseren van de TDM data voor imatinib bleek een hele klus, maar samen met jou gelukkig ook heel gezellig. Julie, bedankt dat je mij altijd wilde helpen met modellen en simulaties en voor je kritische blik op de exposure-respons analyses. Alejandra, fue agradable escribir contigo la reseña sobre los inhibidores de CDK4/6. Además, creo que nunca he completado un manuscrito en tan poco tiempo. Anna, vielen Dank für Ihren Besuch in Amsterdam und für das gemeinsame Schreiben von den Übersichtsartikel über TDM, obwohl dies nicht Teil diese These ist.

Remy, bedankt dat je mij wegwijs hebt willen maken in de wereld van TDM en klinische studies. Hierdoor heb ik een vliegende start kunnen maken. Ook na het afronden van je promotie was je altijd bereid een kritische blik te werpen op mijn manuscripten. Marinda, ik ben heel blij met jou als opvolger en heb er alle vertrouwen in om de TDM-studie aan jou over te dragen. Heel veel succes!

Eline en Lars, tijdens mijn wetenschappelijke stage die ik vier jaar geleden bij jullie in Alkmaar mocht doen ben ik enthousiast geworden om te gaan promoveren. Veel dank voor jullie goede begeleiding destijds!

Alle collega's en oud-collega's van $\mathbf{H 3}$ en uit het O-gebouw, door jullie was promoveren één groot feest. Bedankt voor alle gezelligheid tijdens de lunch, koffiepauzes bij Vermaat, fietstochtjes uit werk, vrijdagmiddagborrels, promotiefeestjes, escalatieborrels, OIOweekenden, OOA retreats in Renesse, congressen, cava dates en natuurlijk onze skireis. Het is gek om in deze tijd afscheid van jullie te nemen, maar ik hoop dat we elkaar nog vaak gaan tegenkomen.

Maaike, heel fijn dat we altijd bij elkaar binnen kunnen lopen om zowel werk-gerelateerde dingen als ons leven daarbuiten te bespreken. Bedankt voor alle gezellige etentjes, borrels 
en de vele sportieve activiteiten die we samen ondernemen. Ik ben heel blij dat je mij deze laatste dag van mijn promotie wilt bijstaan als paranimf.

Gelukkig was er ook naast werk altijd voldoende te beleven. Lieve vrienden en familie, bedankt voor alle mooie momenten. Ik kon altijd uitkijken naar onze gezellige etentjes, borrels, spelletjesavonden, weekendjes weg en vakanties naar de sneeuw of Ibiza. Ik zou niet weten wat ik zonder jullie zou moeten.

Lieve papa en mama, bedankt voor jullie liefdevolle opvoeding, zonder jullie was het nooit gelukt. Jullie hebben me geleerd om altijd mijn best te doen, positief in het leven te staan en in mezelf te geloven. Ik waardeer het enorm dat jullie altijd voor mij klaar staan. Nog altijd voelt het als thuis bij jullie aan de IJvelandssloot! Lieve Anne, ook al wonen we tegenwoordig niet meer bij elkaar om de hoek, des te meer reden om logeerfeestjes te houden in Amstelveen en Den Bosch. Ik ben heel blij dat we altijd alles met elkaar kunnen delen en dat jij vandaag mijn paranimf wilt zijn.

Tot slot, lieve Marco, met jou is het leven zoveel leuker! Bedankt voor jouw onvoorwaardelijke steun en de nodige afleiding de afgelopen jaren, en natuurlijk voor je hulp met de lay-out van dit proefschrift. Samen hebben we al heel veel leuke dingen mogen meemaken en niets overtreft de wereld overreizen samen met jou. Onze plannen voor een wereldreis naar Nieuw-Zeeland en Australië hebben we bijgesteld naar een roadtrip door Europa, waar ik ontzettend naar uit kijk! 


\section{CURRICULUM VITAE}

Steffie Groenland was born on October 20 1991 in Obdam, The Netherlands. In 2010 she graduated from Gymnasium at the Trinitas College, location Han Fortmann in Heerhugowaard. Subsequently, she started studying Medicine at the VU Medical Center in Amsterdam. During this study, she participated in the student-run clinic and coordinated its pharmacovigilance project. As part of her master's program, she performed a research internship at the department of Nephrology of the Northwest Clinics in Alkmaar, focusing on the relation between duration of the prodromal phase and renal damage in ANCA-associated

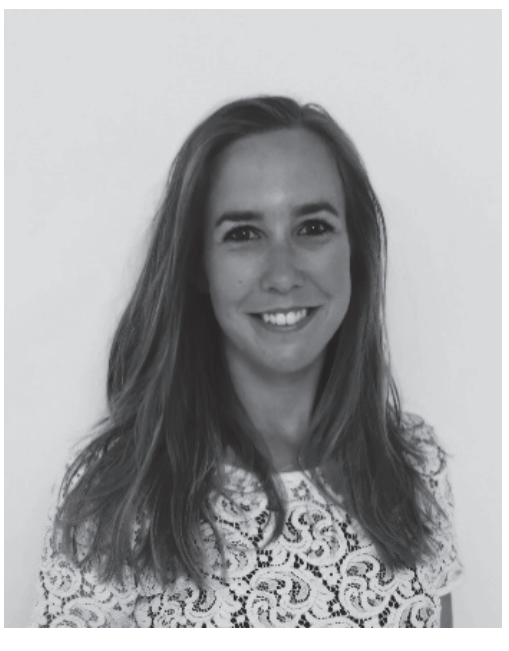
vasculitis. After graduating cum laude in 2016, she started as a PhD candidate at the department of Clinical Pharmacology at the Netherlands Cancer Institute - Antoni van Leeuwenhoek in Amsterdam under supervision of prof. dr. Alwin Huitema, prof. dr. Jos Beijnen and dr. Neeltje Steeghs. Her PhD research focused on precision dosing of oral targeted therapies in oncology and resulted in this thesis. Her main project was setting up and coordinating a large prospective clinical trial in ten hospitals throughout the Netherlands. In 2019 she received an ESMO Merit Award for an oral presentation on this work in Barcelona, Spain. During her PhD research, she also trained as a clinical pharmacologist. In December 2020, she started working as a resident in internal medicine at the Northwest Clinics in Alkmaar. 
